\author{
Universidade de Brasília \\ Instituto de Ciências Exatas \\ Departamento de Matemática
}

\title{
Curvas de Interseção entre duas Superfícies no Espaço Euclidiano e no Espaço de Lorentz-Minkowski
}

por

\section{Lumena Paula de Jesus Borges}

Orientadora: Profa ${ }^{a}$ Dra. Luciana Maria Dias de Ávila Rodrigues

Brasília

2016 
Universidade de Brasília

Instituto de Ciências Exatas

Departamento de Matemática

\section{Curvas de Interseção entre duas Superfícies no Espaço Euclidiano e no Espaço de Lorentz-Minkowski}

por

\section{Lumena Paula de Jesus Borges*}

Dissertação apresentada ao Corpo Docente do Programa de Pós-Graduação

em Matemática - UnB, como requisito parcial para obtenção do grau de

\section{MESTRE EM MATEMÁTICA}

Brasília, 20 de junho de 2016.

Comissão Examinadora:

Prof ${ }^{a}$. Dra. Luciana Maria Dias de Ávila Rodrigues - MAT/UnB (Orientadora)

Prof. Dr. João Paulo dos Santos - MAT/UnB (Membro)

Prof. Dr. Osmar Aléssio - UFTM (Membro)

*A autora foi bolsista CNPq e CAPES durante a elaboração desta dissertação. 


\section{Agradecimentos}

A Deus por me ajudar em todos os momentos.

Aos meus pais, Luiz e Helena, por terem o maior amor e cuidado por mim e aos meus irmãos, Carla e Cleber, pelo apoio que sempre me deram.

Ao meu esposo Valter, um matemático admirável que tem me ajudado de inúmeras formas, desde o início da minha carreira matemática. Sou muito grata a ele por ter sido um companheiro, por ter me encorajado, compartilhado conhecimentos, enfim, por ter colaborado de forma especial para que eu chegasse até aqui.

Meus sinceros agradecimentos à professora Dra. Luciana Ávila que, como sábia orientadora, me conduziu na elaboração deste trabalho.

À Banca examinadora, composta também pelos professores Dr. João Paulo dos Santos e Dr. Osmar Aléssio, que atenciosamente opinaram para melhorias.

Agradeço a cada professor do Departamento de Matemática da UnB que contribuiu direta ou indiretamente, compartilhando suas experiências e seus conhecimentos e apoiando em cada obstáculo que pudesse surgir. Sou grata também aos demais funcionários do Departamento que com carisma e responsabilidade cuidaram de muitos assuntos importantes ao longo destes anos.

Aos meus amigos Ana Paula, Bruno, Chris, Elaine, Erikson, Maria, Nathália, Rafael, Regiane, Sara, Welber e Welinton pelo carinho, companheirismo, pelas horas de estudos compartilhadas, pelos risos, conversas, descontrações e por toda força que têm me dado.

Ao pastor Crislano e à pastora Célia, bem como a toda Igreja Batista Palavra de Vida, pelas orações e amizade.

À CAPES e ao CNPq pelo apoio financeiro. 


\section{Resumo}

Os objetos de estudo nesta dissertação são as curvas de interseção entre duas superfícies no espaço Euclidiano e no espaço de Lorentz-Minkowski. As interseções podem ser do tipo transversal ou tangencial. As superfícies podem ser paramétricas ou implícitas e, portanto, os casos estudados são Paramétrica-Paramétrica, Paramétrica-Implícita e Implícita-Implícita. Quando as superfícies estão no espaço Euclidiano, o objetivo principal é apresentar algoritmos para se obter propriedades geométricas da curva de interseção, tais como curvatura, torção e vetor tangente, em cada caso das interseções. O propósito para o espaço de Lorentz-Minkowski é similar, no qual considera-se curvas de interseção transversal entre duas superfícies tipo espaço, bem como entre duas superfícies tipo tempo, apresentando-se expressões para a curvatura, torção e vetor tangente. Quando as superfícies são tipo espaço, a curva de interseção é também tipo espaço. Quando elas são tipo tempo, a curva pode ser tipo espaço, tipo tempo ou tipo luz. Uma análise para os casos tipo espaço e tipo tempo é feita neste trabalho. Além disso, para superfícies tipo espaço, são dadas condições para que a curva de interseção seja uma geodésica ou uma linha de curvatura das duas superfícies. Exemplos que ilustram esta teoria são acrescentados no final.

Palavras-chave: Interseção de superfícies. Interseção transversal. Interseção tangencial. Espaço de Lorentz-Minkowski. 


\section{Abstract}

The objects of study in this dissertation are the intersection curves of two surfaces in Euclidean space and Lorentz-Minkowski space. Intersections can be of transversal or tangential type. Surfaces can be parametric or implicit and, therefore, the cases studied are ParametricParametric, Parametric-Implicit and Implicit-Implicit. When the surfaces are in Euclidean space, the main objective is presenting algorithms to obtain geometrical properties of the intersection curve, such as curvature, torsion and tangent vector, in each case of the intersections. The purpose for Lorentz-Minkowski space is similar, in which is considered transversal intersection curves between two spacelike surfaces as well as between two timelike surfaces, presenting expressions for the curvature, torsion and tangent vector. When the surfaces are spacelike, the intersection curve is spacelike. When they are timelike, the curve can be spacelike, timelike or lightlike. An analysis for cases spacelike and timelike is considered in this work. Furthermore, for spacelike surfaces, conditions are given so that the intersection is a geodesic curve or line of curvature of both surfaces. Examples illustrating this theory are added at the end.

Keywords: Surface intersection. Transversal intersection. Tangential intersection. LorentzMinkowski space. 


\section{Sumário}

$\begin{array}{ll}\text { Introdução } & 1\end{array}$

1 Preliminares $\quad 3$

$1.1 \mathrm{O}$ Espaço Euclidiano $\mathbb{R}^{3} \ldots \ldots \ldots \ldots \ldots \ldots \ldots$

1.1.1 Curvas Paramétricas . . . . . . . . . . . . . . . . . 3

1.1.2 Superfícies Paramétricas e Implícitas . . . . . . . . . . . . . . . . 5

1.2 O Espaço de Lorentz-Minkowski $\mathbb{L}^{3} \ldots \ldots \ldots \ldots$

1.2.1 Propriedades e Definições Básicas . . . . . . . . . . . . . . 14

1.2.2 Curvas Paramétricas . . . . . . . . . . . . . . . . 27

1.2.3 Superfícies Paramétricas . . . . . . . . . . . . . . . . . . 32

2 Curvas de Interseção entre duas Superfícies no Espaço Euclidiano 36

2.1 Interseção Transversal . . . . . . . . . . . . . . . . . . . 36

2.1.1 Interseção entre duas Superfícies Paramétricas . . . . . . . . . . . . 36

2.1.2 Interseção entre Superfícies Paramétricas e Implícitas . . . . . . . . . . . . 42

2.1.3 Interseção entre duas Superfícies Implícitas . . . . . . . . . . . . . . . 47

2.2 Interseção Tangencial . . . . . . . . . . . . . . . . . . . . 60

2.2.1 Interseção entre duas Superfícies Paramétricas . . . . . . . . . . . . . 60

2.2.2 Interseção entre Superfícies Paramétricas e Implícitas . . . . . . . . . . . 72

2.2.3 Interseção entre duas Superfícies Implícitas . . . . . . . . . . . . . . . 77

3 Curvas de Interseção entre duas Superfícies no Espaço de Lorentz-Minkowski 83

3.1 Interseção Transversal entre duas Superfícies Paramétricas Tipo Espaço . . . . . . 84

3.2 Interseção Transversal Não-Nula entre duas Superfícies Paramétricas Tipo Tempo 90 
4.1 Exemplos no Espaço Euclidiano . . . . . . . . . . . . . . . . . . 95

4.2 Exemplos no Espaço de Lorentz-Minkowski . . . . . . . . . . . . . . . . 102 


\section{Introdução}

Em sistemas de modelagem geométrica, é comum que se trabalhe com superfícies paramétricas e implícitas, gerando três tipos de interseção a serem estudadas: Paramétrica-Paramétrica, Paramétrica-Implícita e Implícita-Implícita. A interseção pode ser transversal (os vetores normais não são paralelos) ou tangencial (os vetores normais são paralelos). Ao considerarmos interseções entre superfícies arbitrárias, pode não ser simples obter a parametrização, bem como propriedades básicas da curva de interseção. Poderíamos então nos perguntar o que fazer ao nos depararmos com problemas que envolvam curvas que resultam da interseção entre duas superfícies e que suas parametrizações não sejam conhecidas. Veremos no decorrer deste trabalho alguns métodos que nos permitem conhecer propriedades como curvatura, torção e outros entes geométricos em um dado ponto da curva de interseção. Tais entes serão obtidos através das expressões das superfícies, sejam elas paramétricas ou implícitas. Esta teoria pode ser aplicada, por exemplo, à geometria computacional e modelagem geométrica de formas complexas em sistemas CAD/CAM. Em geral, métodos numéricos são usados para a obtenção da curva de interseção, dos quais o mais comum é o método de caminhada. Tal método envolve sequência de pontos sobre a curva de interseção, obtidos através da geometria diferencial local da mesma [18].

No espaço Euclidiano, Ye e Maekawa [18, 1999] apresentaram algoritmos para calcular as propriedades geométricas da curva de interseção transversal e tangencial de duas superfícies paramétricas e descreveram como obter estas propriedades para o caso Paramétrica-Implícita e Implícita-Implícita, além de darem algoritmos para avaliar as derivadas de ordem alta da curva de interseção, as quais são importantes para que, utilizando alguns métodos, se obtenha uma boa aproximação da curva. Aléssio $[4,2006]$ apresentou uma técnica para o caso ImplícitaImplícita, usando-se o Teorema da Função Implícita, técnica tal que, independentemente da ordem das derivadas que estejam envolvidas nos cálculos, permite que os entes geométricos da curva sejam obtidos a partir da resolução de sistemas lineares de duas equações e duas variáveis. Soliman et al. [14, 2011] aborda o caso Paramétrica-Implícita e Abdel-All et al. [1, 2012] também considera o caso Implícita-Implícita tratado com o Teorema da Função Implícita, apresentando condições necessárias e suficientes para que a curva de interseção seja uma reta, curva plana, hélice, hélice circular ou um círculo. 
Nos últimos anos, tem crescido o estudo sobre as curvas de interseção entre superfícies no espaço de Lorentz-Minkowski. Neste ambiente, os casos a serem analisados aumentam, visto que, a cada tipo de interseção (Paramétrica-Paramétrica, Paramétrica-Implícita e ImplícitaImplícita), deve-se ainda analisar o caráter de causalidade das superfícies, bem como da curva de interseção, isto é, se elas são tipo espaço, tipo tempo ou tipo luz. Aléssio e Guadalupe [3, 2007] estudaram as curvas de interseção transversal entre duas superfícies paramétricas tipo espaço. Neste caso, a curva de interseção $\alpha$ resulta tipo espaço. Eles apresentaram condições para que $\alpha$ seja uma geodésica ou uma linha de curvatura das duas superfícies. Já Şanli e Yayli $[12,2014]$ expressaram a curvatura e a torção da curva de interseção transversal entre duas superfícies paramétricas tipo tempo, $\operatorname{com} \alpha$ tipo espaço ou tipo tempo.

Neste trabalho, estudamos os métodos apresentados em [1], [3], [12], [14] e [18]. No Capítulo 1, fizemos uma revisão sobre curvas e superfícies no espaço Euclidiano, apresentamos as propriedades e definições básicas do espaço de Lorentz-Minkowski e fizemos um breve estudo sobre as curvas e superfícies neste espaço. O Capítulo 2 foi dividido em duas seções que tratam, respectivamente, das interseções transversal e tangencial no espaço Euclidiano. Consideramos tais interseções para os três casos: Paramétrica-Paramétrica, Paramétrica-Implícita e Implícita-Implícita, exibindo métodos para calcular as propriedades geométricas da curva de interseção, tais como curvatura, torção, vetor tangente e vetor curvatura. No caso de interseção tangencial entre duas superfícies paramétricas, é feita uma interpretação geométrica do vetor tangente num ponto $P$ da interseção em termos das indicatrizes de Dupin das duas superfícies em $P$. No Capítulo 3, consideramos as curvas de interseção transversal entre duas superfícies no espaço de Lorentz-Minkowski, onde abordamos as interseções entre duas superfícies tipo espaço, bem como entre duas superfícies tipo tempo. Além disso, apresentamos alguns exemplos no Capítulo 4, visando ilustrar a teoria estudada. 


\section{Capítulo 1}

\section{Preliminares}

O presente capítulo tem por objetivo relembrar ou introduzir conceitos relacionados aos espaços Euclidiano e de Lorentz-Minkowski. A métrica do espaço de Lorentz-Minkowski difere da métrica do espaço Euclidiano por um único sinal, ocasionando grandes mudanças como, por exemplo, a existência de vetores que são ortogonais se, e somente se, são colineares. Veremos a seguir conceitos relacionados a estes espaços com o objetivo de estudar as curvas e superfícies nestes.

\subsection{O Espaço Euclidiano $\mathbb{R}^{3}$}

Os conceitos de curvas paramétricas e superfícies paramétricas e implícitas serão muito importantes para o desenvolvimento dos próximos capítulos deste trabalho. Assim, será feito nesta seção um breve estudo das propriedades geométricas das curvas e superfícies no espaço Euclidiano $\mathbb{R}^{3}$, tendo [2], [5], [15] e [16] como principais referências. Na Subseção 1.1.1, falamos sobre as curvas paramétricas e, na Subseção 1.1.2, considera-se as superfícies paramétricas e implícitas.

\subsubsection{Curvas Paramétricas}

Faremos uma revisão sobre as curvas paramétricas (ou parametrizadas) diferenciáveis no espaço Euclidiano $\mathbb{R}^{3}$. Diremos que uma aplicação é diferenciável se ela possui derivadas de todas as ordens.

Definição 1.1. Uma curva parametrizada diferenciável em $\mathbb{R}^{3}$ é uma aplicação $\alpha: I \rightarrow \mathbb{R}^{3}$ definida no intervalo aberto $I \subset \mathbb{R}$ que édiferenciável. 
Denotaremos a curva $\alpha$ por $\alpha(t)=(x(t), y(t), z(t))$. Dessa forma, são diferenciáveis as funções $x(t), y(t), z(t)$. O vetor $\alpha^{\prime}(t)=\left(x^{\prime}(t), y^{\prime}(t), z^{\prime}(t)\right)$ é chamado $o$ vetor tangente a $\alpha$ em $t \mathrm{e}$ a imagem $\alpha(I) \subset \mathbb{R}^{3}$ é chamada o traço da curva $\alpha$.

Definição 1.2. Dizemos que uma curva $\alpha$ é regular se $\alpha^{\prime}(t) \neq 0$, para todo $t \in I$.

Trataremos sempre de curvas parametrizadas diferenciáveis regulares e, por comodidade, faremos referência a tais curvas sem estes adjetivos.

Dado $t_{0} \in I$, o comprimento de arco de $\alpha$ a partir de $t_{0}$ é definido por

$$
S(t)=\int_{t_{0}}^{t}\left\|\alpha^{\prime}(u)\right\| d u
$$

Dizemos que $\alpha$ está parametrizada pelo comprimento de arco se $\left\|\alpha^{\prime}(t)\right\|=1$. Sempre existe uma reparametrização de $\alpha$ pelo comprimento de arco, isto é, existe uma curva $\beta: J \rightarrow \mathbb{R}^{3}$ que tem o mesmo traço que $\alpha$ e satisfaz $\left\|\beta^{\prime}(s)\right\|=1$, onde $s=S(t)$ é o comprimento de arco de $\alpha$. Por este motivo, nos restringiremos ao estudo das curvas parametrizadas pelo comprimento de arco.

Definição 1.3. Seja $\alpha$ uma curva parametrizada pelo comprimento de arco. Definimos a curvatura de $\alpha$ pelo número real

$$
\kappa(s)=\left\|\alpha^{\prime \prime}(s)\right\|
$$

Se $\kappa \neq 0$, definimos os vetores normal e binormal em $s$ por $n(s)=\frac{\alpha^{\prime \prime}(s)}{\kappa(s)}$ e $b(s)=\alpha^{\prime}(s) \wedge n(s)$, respectivamente.

Consideraremos apenas curvas com curvatura não nula. Denotaremos $t=\alpha^{\prime}$. Desse modo, para cada ponto $s \in I$, ficam definidos os vetores normais unitários $t(s), n(s), b(s)$. O triedro assim construído é chamado o triedro de Frenet de $\alpha$ em s. O plano gerado por $t$ e $n$ é chamado o plano osculador em s, o plano gerado por $t$ e $b$ é o plano retificante e o plano gerado por $n$ e $b$ é chamado plano normal.

Da definição do vetor normal, temos que $t^{\prime}=\kappa n$. Observe que, por um lado, sendo $b$ unitário, tem-se que $b^{\prime}$ é ortogonal a $b$. Por outro lado,

$$
b^{\prime}=t^{\prime} \wedge n+t \wedge n^{\prime}=\kappa n \wedge n+t \wedge n^{\prime}=t \wedge n^{\prime},
$$

isto é, $b^{\prime}$ é ortogonal a $t$. Logo, $b^{\prime}$ é paralelo a $n$ e, portanto, existe uma função $\tau$ que satisfaz

$$
b^{\prime}(s)=-\tau(s) n(s)
$$

Definição 1.4. Seja $\alpha$ uma curva parametrizada pelo comprimento de arco s tal que $\alpha^{\prime \prime}(s) \neq 0$. $O$ número $\tau(s)$ definido por $b^{\prime}(s)=-\tau(s) n(s)$ é chamado a torção de $\alpha$ em s. 
Note ainda que, como $n=b \wedge t$, então

$$
n^{\prime}=b^{\prime} \wedge t+b \wedge t^{\prime}=-\tau n \wedge t+\kappa b \wedge n=\tau b-\kappa t .
$$

Chamamos as equações de Frenet às seguintes expressões:

$$
\left\{\begin{array}{l}
t^{\prime}=\kappa n \\
n^{\prime}=-\kappa t+\tau b \\
b^{\prime}=-\tau n .
\end{array}\right.
$$

No caso de $\alpha$ depender de um parâmetro $t$ que não necessariamente é o comprimento de arco, pode-se mostrar que

$$
\begin{aligned}
\tau(t) & =\frac{\left\langle\alpha^{\prime} \wedge \alpha^{\prime \prime}, \alpha^{\prime \prime \prime}\right\rangle}{\left\|\alpha^{\prime} \wedge \alpha^{\prime \prime}\right\|^{2}}, \kappa \neq 0 \\
\kappa(t) n(t) & =\frac{\left\langle\alpha^{\prime}, \alpha^{\prime}\right\rangle \alpha^{\prime \prime}-\left\langle\alpha^{\prime}, \alpha^{\prime \prime}\right\rangle \alpha^{\prime}}{\left\|\alpha^{\prime}\right\|^{4}} \\
\kappa(t) & =\frac{\left\|\alpha^{\prime} \wedge \alpha^{\prime \prime}\right\|}{\left\|\alpha^{\prime}\right\|^{3}} .
\end{aligned}
$$

\subsubsection{Superfícies Paramétricas e Implícitas}

Trataremos nesta seção de alguns conceitos geométricos das superfícies paramétricas e implícitas. Esta é uma teoria clássica e já explícita em muitos livros e relembraremos aqui alguns conceitos básicos. As principais fontes de referência são [2], [5], [15] e [16]. Para o que segue, é conveniente definir o conceito de diferencial de uma aplicação. Diremos que uma aplicação é diferenciável se ela é de classe $C^{\infty}$.

Dada uma aplicação diferenciável $f: U \subset \mathbb{R}^{n} \rightarrow \mathbb{R}^{m}$, associamos a cada $p \in U$ uma aplicação linear $d f_{p}: \mathbb{R}^{n} \rightarrow \mathbb{R}^{m}$ que é chamada a diferencial de $f$ em $p$ e é definida da seguinte maneira. Sejam $w \in \mathbb{R}^{n}$ e $\alpha: I \subset \mathbb{R} \rightarrow U$ uma curva diferenciável tal que $\alpha(0)=p$ e $\alpha^{\prime}(0)=w$. Pela regra da cadeia, a curva $\beta=f \circ \alpha: I \rightarrow \mathbb{R}^{m}$ também é diferenciável. Define-se

$$
d f_{p}(w)=\beta^{\prime}(0)
$$

Definição 1.5. Uma superfície paramétrica regular em $\mathbb{R}^{3}$ é uma aplicação $X: U \rightarrow \mathbb{R}^{3}$, definida no aberto $U$ do $\mathbb{R}^{2}$, tal que X é diferenciável e, para todo $q=(u, v) \in U$, a diferencial $d X_{q}: \mathbb{R}^{2} \rightarrow \mathbb{R}^{3}$ é injetiva.

Note que a última condição na Definição 1.5 equivale a dizer que $X_{u} \wedge X_{v} \neq 0$. O conjunto $X(U)$ é chamado o traço de $X$.

Dizemos que $S \subset \mathbb{R}^{3}$ é uma superfície regular se, para cada $p \in S$, existe uma vizinhança $V$ de $p$ em $\mathbb{R}^{3}$ e uma aplicação $X: U \rightarrow V \cap S$ de um aberto $U$ do $\mathbb{R}^{2}$ sobre $V \cap S$ tal que $X$ é um homeomorfismo diferenciável e a diferencial $d X_{q}$ é injetiva, para todo $q \in U$. 
Observe que a definição de superfície paramétrica permite que seu traço tenha auto interseção. Mas, neste trabalho, não consideraremos casos assim. Uma superfície paramétrica é também chamada superfície parametrizada.

Os dois conceitos introduzidos tratam de um mesmo objeto de dois pontos de vista distintos e que estão relacionados de alguma maneira. Em [5], podemos ver que, dada uma superfície paramétrica regular $X: U \rightarrow \mathbb{R}^{3}$, seu traço localmente é uma superfície regular. Além disso, uma parametrização $X$ de uma superfície regular é uma superfície paramétrica. Motivados por isto e pelo fato de o nosso estudo ser local, identificaremos as duas definições, isto é, uma superfície paramétrica com o seu traço, bem como uma superfície regular com a sua parametrização, conforme seja conveniente.

Dada uma superfície parametrizada $X$ e um ponto $p=X(q)$, o espaço vetorial

$$
T_{p} X:=d X_{q}\left(\mathbb{R}^{2}\right)
$$

é o plano tangente a X em $p$. O vetor normal unitário da superfície em $p$ é dado por

$$
N(p)=\frac{X_{u} \wedge X_{v}}{\left\|X_{u} \wedge X_{v}\right\|}(q)
$$

Variando o ponto $p \in X(U)$, obtemos uma aplicação diferenciável $N: X(U) \rightarrow \mathbb{R}^{3}$.

Como trataremos nos capítulos seguintes de curvas de interseção entre superfícies, encontraremos algumas expressões relevantes para este fim. A curva planar $s \mapsto(u(s), v(s))$ define uma curva $\alpha(s)=X(u(s), v(s))$ sobre a superfície paramétrica $X(u, v)$. Derivando esta expressão com respeito a $s$, segue que

$$
\begin{aligned}
\alpha^{\prime}= & X_{u} u^{\prime}+X_{v} v^{\prime} \\
\alpha^{\prime \prime}= & X_{u u}\left(u^{\prime}\right)^{2}+2 X_{u v} u^{\prime} v^{\prime}+X_{v v}\left(v^{\prime}\right)^{2}+X_{u} u^{\prime \prime}+X_{v} v^{\prime \prime} \\
\alpha^{\prime \prime \prime}= & X_{u u u}\left(u^{\prime}\right)^{3}+3 X_{u u v}\left(u^{\prime}\right)^{2} v^{\prime}+3 X_{u v v} u^{\prime}\left(v^{\prime}\right)^{2}+X_{v v v}\left(v^{\prime}\right)^{3}+ \\
& 3\left[X_{u u} u^{\prime} u^{\prime \prime}+X_{u v}\left(u^{\prime \prime} v^{\prime}+u^{\prime} v^{\prime \prime}\right)+X_{v v} v^{\prime} v^{\prime \prime}\right]+X_{u} u^{\prime \prime \prime}+X_{v} v^{\prime \prime \prime},
\end{aligned}
$$

onde omitimos o parâmetro s por simplicidade.

Falaremos um pouco sobre a primeira forma fundamental, que é definida a partir do produto interno usual do $\mathbb{R}^{3}$, o qual induz um produto interno em cada plano tangente $T_{p} X$ de uma superfície paramétrica $X$. Dados $w_{1}, w_{2} \in X(U)$, definimos $\left\langle w_{1}, w_{2}\right\rangle_{p}=\left\langle w_{1}, w_{2}\right\rangle$, onde $\langle$, denota o produto interno usual do $\mathbb{R}^{3}$. A esse produto interno, corresponde uma forma quadrática $I_{p}: T_{p} X \rightarrow \mathbb{R}$ dada por

$$
I_{p}(w)=\langle w, w\rangle_{p} \geq 0
$$

Definição 1.6. A forma quadrática $I_{p}$ definida em (1.5) é chamada a primeira forma fundamental da superfície $X$ no ponto $p \in X(U)$. 
Vamos agora expressar a primeira forma fundamental na base $\left\{X_{u}, X_{v}\right\}$. Dado $w \in T_{p} X$, podemos escrever $w=\alpha^{\prime}(0)$, onde $\alpha(t)=X(u(t), v(t))$ é uma curva sobre a superfície $X$ tal que $\alpha(0)=p$. Temos que

$$
\begin{aligned}
I_{p}(w) & =I_{p}\left(\alpha^{\prime}(0)\right)=\left\langle\alpha^{\prime}(0), \alpha^{\prime}(0)\right\rangle_{p} \\
& =\left\langle X_{u} u^{\prime}+X_{v} v^{\prime}, X_{u} u^{\prime}+X_{v} v^{\prime}\right\rangle_{p} \\
& =E\left(u^{\prime}\right)^{2}+2 F u^{\prime} v^{\prime}+G\left(v^{\prime}\right)^{2}
\end{aligned}
$$

onde as funções são calculadas em $t=0 \mathrm{e}$

$$
\begin{aligned}
& E=E\left(u_{0}, v_{0}\right)=\left\langle X_{u}, X_{u}\right\rangle_{p} \\
& F=F\left(u_{0}, v_{0}\right)=\left\langle X_{u}, X_{v}\right\rangle_{p} \\
& G=G\left(u_{0}, v_{0}\right)=\left\langle X_{v}, X_{v}\right\rangle_{p}
\end{aligned}
$$

são os coeficientes da primeira forma fundamental na base $\left\{X_{u}, X_{v}\right\}$ de $T_{p} X$. Fazendo $p \in X(U)$ variar, obtemos funções $E(u, v), F(u, v), G(u, v)$ diferenciáveis. De agora em diante, omitiremos o subscrito $p$ quando for claro pelo contexto a que ponto nos referimos.

Definição 1.7. Seja X uma superfície com uma orientação determinada pelo campo de vetores normais unitários N. A aplicação $N: X(U) \rightarrow \mathbb{R}^{3}$ toma seus valores na esfera unitária $\mathrm{S}^{2}$. A aplicação $N: X(U) \rightarrow S^{2}$ é chamada a aplicação de Gauss de X, a qual é uma aplicação diferenciável.

Pode-se provar que a diferencial $d N_{p}: T_{p} X \rightarrow T_{p} X$ é uma aplicação linear auto-adjunta, o que nos permite associar a $d N_{p}$ uma forma quadrática $v \mapsto\left\langle d N_{p}(v), v\right\rangle$ em $T_{p} X$.

Definição 1.8. A forma quadrática $I I_{p}: T_{p} X \rightarrow \mathbb{R}$ dada por $I I_{p}(v)=-\left\langle d N_{p}(v)\right.$, v $\rangle$ é chamada $a$ segunda forma fundamental de $X$ em $p \in X(U)$.

Definição 1.9. Seja a uma curva regular sobre a superfície $X$ que passa pelo ponto $p$. A curvatura normal de $\alpha$ em $p$ é o número real $\kappa_{n}=\langle\vec{K}, N\rangle$, onde $\vec{K}=\alpha^{\prime \prime}$ é o vetor curvatura de $\alpha$. Assim,

$$
\kappa_{n}=\langle\vec{K}, N\rangle=\kappa\langle n, N\rangle=\kappa \cos \theta
$$

onde $\kappa$ e $n$ são a curvatura e o vetor normal de a em p e $\theta$ o ângulo entre os normais ne $N$.

Vamos dar uma interpretação geométrica da segunda forma fundamental em termos da curvatura normal. Dado um vetor unitário $v \in T_{p} X$, existe uma curva $\alpha$ parametrizada pelo comprimento de arco $s$ tal que $\alpha(0)=p$ e $\alpha^{\prime}(0)=v$. Denote por $N(s)$ a restrição do vetor normal $N$ à curva $\alpha(s)$. Temos que $\left\langle N(s), \alpha^{\prime}(s)\right\rangle=0$, o que implica $\left\langle N(s), \alpha^{\prime \prime}(s)\right\rangle=-\left\langle N^{\prime}(s), \alpha^{\prime}(s)\right\rangle$. 
Portanto,

$$
\begin{aligned}
I I_{p}(v) & =I I_{p}\left(\alpha^{\prime}(0)\right)=-\left\langle d N_{p}\left(\alpha^{\prime}(0)\right), \alpha^{\prime}(0)\right\rangle \\
& =-\left\langle N^{\prime}(0), \alpha^{\prime}(0)\right\rangle=\left\langle N(0), \alpha^{\prime \prime}(0)\right\rangle \\
& =\langle N, \kappa n\rangle(p)=\kappa_{n}(p) .
\end{aligned}
$$

Daí, temos a seguinte proposição.

Proposição 1.1 (Meusnier). Todas as curvas de uma superfície $X: U \subset \mathbb{R}^{2} \rightarrow \mathbb{R}^{3}$ que têm, em um ponto $p \in X(U)$, a mesma reta tangente, têm, neste ponto, a mesma curvatura normal.

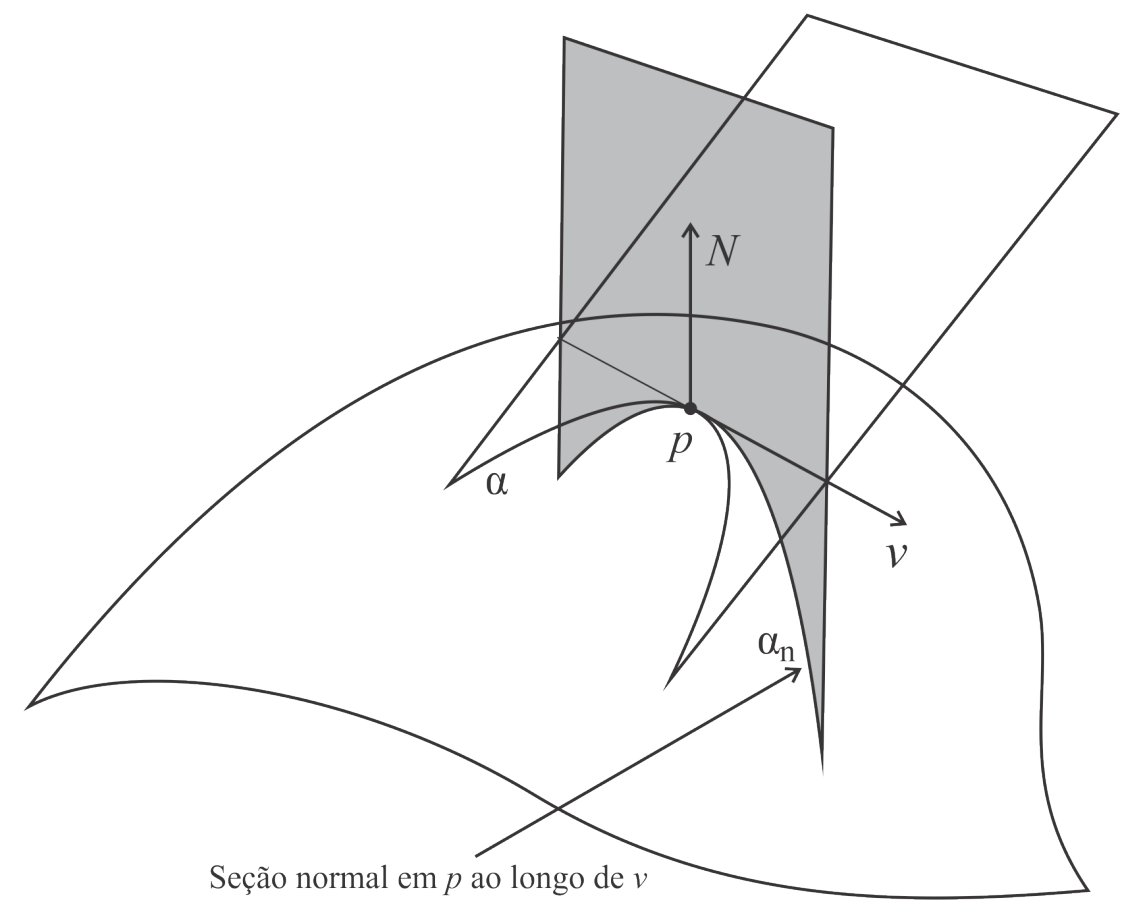

Figura 1.1: $\alpha$ e $\alpha_{n}$ têm a mesma curvatura normal em $p$ ao longo de $v$

Deste ponto de vista, podemos falar em curvatura normal ao longo de uma dada direção. Chamamos a seção normal de X em $p$ segundo $v$ à interseção de $X$ com um plano contendo $v \mathrm{e}$ $N(p)$. Em uma vizinhança de $p$, a seção normal é uma curva plana regular cujo vetor normal é $\pm N(p)$ ou zero. Assim, sendo $\kappa_{n}=\kappa\langle n, N\rangle$, temos $\kappa= \pm \kappa_{n}$, isto é, o valor absoluto da curvatura normal é igual à curvatura da seção normal de $X$ em $p$, segundo $\alpha^{\prime}(0)$.

Pode-se mostrar que, para cada $p \in X(U)$, existe uma base ortonormal $\left\{e_{1}, e_{2}\right\}$ de $T_{p} X$ tal que

$$
d N_{p}\left(e_{1}\right)=-\kappa_{1} e_{1}, \quad d N_{p}\left(e_{2}\right)=-\kappa_{2} e_{2},
$$

com $\kappa_{1}$ e $\kappa_{2}$ sendo o máximo e o mínimo da segunda forma fundamental (ou da curvatura normal) restrita ao círculo unitário de $T_{p} X$. Convencionaremos $\kappa_{1} \leq \kappa_{2}$. Os vetores $e_{1}$ e $e_{2}$ 
são chamados vetores principais de $X$ em $p$, as direções determinadas por eles são as direções principais e as curvaturas $\kappa_{1}$ e $\kappa_{2}$ são as curvaturas principais.

Definição 1.10. Seja $p \in X(U)$ e $d N_{p}: T_{p} X \rightarrow T_{p} X$ a diferencial da aplicação de Gauss.

1. A curvatura Gaussiana de $X$ em $p$ é definida por $K=\operatorname{det}\left(d N_{p}\right)$.

2. A curvatura média de $X$ em $p$ é $H=-\frac{1}{2} \operatorname{tr}\left(d N_{p}\right)$, onde tr representa o traço da matriz da aplicação.

Segue, portanto, que

$$
K(p)=\kappa_{1} \kappa_{2}, \quad H(p)=\frac{\kappa_{1}+\kappa_{2}}{2} .
$$

Definição 1.11. Um ponto $p \in X(U)$ é dito

1. Eliptico, se $\operatorname{det}\left(d N_{p}\right)>0$.

2. Hiperbólico, se $\operatorname{det}\left(d N_{p}\right)<0$.

3. Parabólico, se $\operatorname{det}\left(d N_{p}\right)=0$, com $d N_{p} \neq 0$.

4. Planar, se $d N_{p} \equiv 0$.

Temos ainda a seguinte definição.

Definição 1.12. Dizemos que um ponto $p \in X(U)$ é umbílico se $\kappa_{1}=\kappa_{2}$.

Veremos a seguir como as curvaturas principais determinam a curvatura normal em qualquer direção. Seja $w \in T_{p} X$ com $\|w\|=1$ e $e_{1}, e_{2}$ os vetores principais da superfície $X$ em $p$. Como $e_{1}$ e $e_{2}$ formam uma base ortonormal de $T_{p} X$, podemos escrever

$$
w=\cos \theta e_{1}+\sin \theta e_{2}
$$

onde $\theta$ é o ângulo de $e_{1}$ a $w$ na orientação de $T_{p} X$. A curvatura normal $\kappa_{n}$ na direção de $w$ é dada por

$$
\begin{aligned}
\kappa_{n}(w) & =I I_{p}(w)=-\left\langle d N_{p}\left(\cos \theta e_{1}+\sin \theta e_{2}\right), \cos \theta e_{1}+\sin \theta e_{2}\right\rangle \\
& =-\left\langle-\kappa_{1} \cos \theta e_{1}-\kappa_{2} \sin \theta e_{2}, \cos \theta e_{1}+\sin \theta e_{2}\right\rangle \\
& =\kappa_{1} \cos ^{2} \theta+\kappa_{2} \sin ^{2} \theta .
\end{aligned}
$$

Assim,

$$
\kappa_{n}=\kappa_{1} \cos ^{2} \theta+\kappa_{2} \sin ^{2} \theta
$$

Esta expressão é conhecida como fórmula de Euler. Ela nos dá uma expressão para a segunda forma fundamental $I I_{p}(w)=\kappa_{n}(w)$ na base de autovetores $\left\{e_{1}, e_{2}\right\}$ de $T_{p} X$. 
Definição 1.13. Dizemos que uma curva a regular e conexa sobre uma superfície $X$ é uma linha de curvatura se, para todo $p$ sobre $\alpha$, a reta tangente a $\alpha$ é uma direção principal.

Proposição 1.2 (Olinde Rodrigues). Uma condição necessária e suficiente para que uma curva regular e conexa a seja uma linha de curvatura de Xé

$$
N^{\prime}(t)=\lambda(t) \alpha^{\prime}(t)
$$

onde $\lambda(t)$ é uma função diferenciável e $N(t)=N \circ \alpha(t)$. Neste caso, $-\lambda(t)$ é a curvatura principal segundo $\alpha^{\prime}(t)$.

Demonstração. Se $\alpha$ é uma linha de curvatura, então $\alpha^{\prime}(t)$ está contido em uma direção principal. Logo, $\alpha^{\prime}(t)$ é um autovetor de $d N$ e, assim, para alguma função $\lambda$ de $t$,

$$
N^{\prime}(t)=d N\left(\alpha^{\prime}(t)\right)=\lambda(t) \alpha^{\prime}(t) .
$$

Reciprocamente, se $N^{\prime}(t)=\lambda(t) \alpha^{\prime}(t)$, segue que $\alpha^{\prime}(t)$ é um autovetor de $d N$ e, portanto, está contido numa direção principal, ou seja, $\alpha$ é uma linha de curvatura.

Definição 1.14. Seja $p \in X(U)$. Uma direção assintótica é uma direção de $T_{p} X$ para a qual a curvatura normal é zero. Uma curva conexa e regular $\alpha$ é dita curva assintótica de $X$ se, para todo $p$ sobre $\alpha$, a reta tangente a a em p é uma direção assintótica.

Vamos falar agora sobre a indicatriz de Dupin. Dado um ponto $p \in X(U)$, a indicatriz de Dupin em $p$ é o conjunto de vetores $w \in T_{p} X$ que satisfazem $I I_{p}(w)= \pm 1$. Podemos ver que a indicatriz de Dupin em $p$ é uma união de cônicas. De fato, sejam $\xi$ e $\eta$ as coordenadas cartesianas de $T_{p} X$ na base ortonormal $\left\{e_{1}, e_{2}\right\}$, onde $e_{1}, e_{2}$ são autovetores da $d N_{p}$. Dado $w \in T_{p} X$, escreva $w=\xi e_{1}+\eta e_{2}$. Considere as coordenadas polares $\xi=\rho \cos \theta, \eta=\rho \sin \theta$, $\rho \neq 0$. Sendo $\|v\|=1$ e $\left\{e_{1}, e_{2}\right\}$ base ortonormal, podemos escrever $v=\cos \theta e_{1}+\sin \theta e_{2}$. Assim,

$$
w=\rho \cos \theta e_{1}+\rho \sin \theta e_{2}=\rho v .
$$

Portanto, $w \in T_{p} X$ satisfaz $I I_{p}(w)= \pm 1$ se, e somente se,

$$
\begin{aligned}
\pm 1 & =I I_{p}(w)=I I_{p}(\rho v)=-\left\langle d N_{p}(\rho v), \rho v\right\rangle \\
& =\rho^{2}\left[-\left\langle d N_{p}(v), v\right\rangle\right]=\rho^{2} I I_{p}(v) \\
& =\rho^{2}\left(\kappa_{1} \cos ^{2} \theta+\kappa_{2} \sin ^{2} \theta\right) \\
& =\kappa_{1} \xi^{2}+\kappa_{2} \eta^{2},
\end{aligned}
$$

onde, na penúltima igualdade, usamos a fórmula de Euler (ver equação (1.7)). Concluímos que as coordenadas $\xi$ e $\eta$ de um ponto da indicatriz de Dupin satisfazem

$$
\kappa_{1} \xi^{2}+\kappa_{2} \eta^{2}= \pm 1
$$


ou seja, a indicatriz de Dupin em $p$ é a união das cônicas $\kappa_{1} \xi^{2}+\kappa_{2} \eta^{2}=1$ e $\kappa_{1} \xi^{2}+\kappa_{2} \eta^{2}=-1$ em $T_{p} X$. Observe que, se $p$ é um ponto planar, a equação (1.8) não é satisfeita. Analisaremos o que acontece nos outros casos.

1. Se $p$ é um ponto elíptico, $\kappa_{1} \kappa_{2}>0$ e, então, $\kappa_{1}$ e $\kappa_{2}$ têm o mesmo sinal. Logo, por (1.8), a indicatriz de Dupin é uma elipse, que degenera em um círculo se o ponto é umbílico.

2. Se $p$ é um ponto hiperbólico, $\kappa_{1} \kappa_{2}<0$. Assim, $\kappa_{1}$ e $\kappa_{2}$ têm sinais opostos. Logo, analisando cada caso $\left(\kappa_{1}>0, \kappa_{2}<0\right.$ e $\left.\kappa_{1}<0, \kappa_{2}>0\right)$, segue de (1.8) que a indicatriz de Dupin é um par de hipérboles que não se tocam.

3. Se $p$ é parabólico, então uma das curvaturas principais é zero. Logo, a indicatriz de Dupin neste caso é um par de retas paralelas, com direção comum igual à única direção assintótica em $p$.

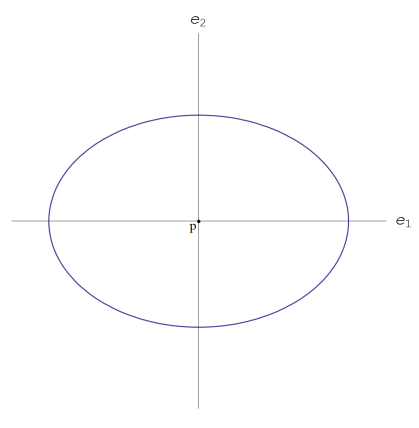

(a) O ponto $p$ é elíptico

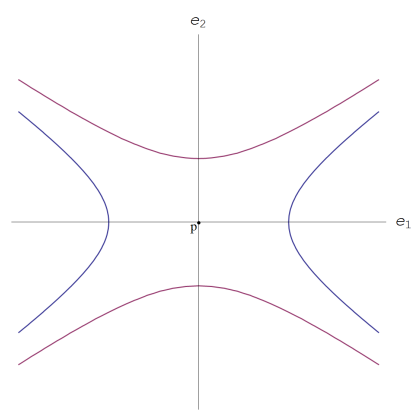

(b) O ponto $p$ é hiperbólico

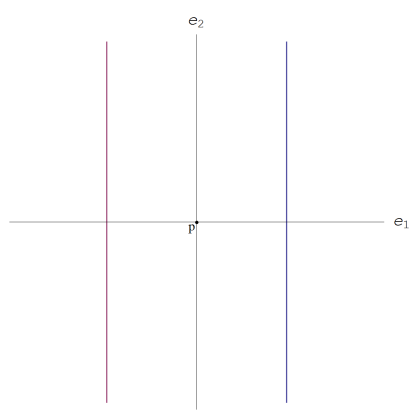

(c) O ponto $p$ é parabólico

Figura 1.2: Indicatrizes de Dupin no ponto $p$

Nosso objetivo agora é encontrar uma expressão para a segunda forma fundamental em um sistema de coordenadas locais. Seja $X(u, v)$ uma superfície, $p \in X(U)$ e $\alpha(t)=X(u(t), v(t))$ uma curva sobre $X$ tal que $\alpha(0)=p$. Observe que

$$
d N\left(\alpha^{\prime}\right)=N^{\prime}(u(t), v(t))=N_{u} u^{\prime}+N_{v} v^{\prime} .
$$

Portanto,

$$
\begin{aligned}
I I_{p}\left(\alpha^{\prime}\right) & =-\left\langle d N\left(\alpha^{\prime}\right), \alpha^{\prime}\right\rangle=-\left\langle N_{u} u^{\prime}+N_{v} v^{\prime}, X_{u} u^{\prime}+X_{v} v^{\prime}\right\rangle \\
& =-\left[\left\langle N_{u}, X_{u}\right\rangle\left(u^{\prime}\right)^{2}+\left(\left\langle N_{u}, X_{v}\right\rangle+\left\langle N_{v}, X_{u}\right\rangle\right) u^{\prime} v^{\prime}+\left\langle N_{v}, X_{v}\right\rangle\left(v^{\prime}\right)^{2}\right] \\
& =e\left(u^{\prime}\right)^{2}+2 f u^{\prime} v^{\prime}+g\left(v^{\prime}\right)^{2},
\end{aligned}
$$


onde

$$
\begin{aligned}
& e=-\left\langle N_{u}, X_{u}\right\rangle=\left\langle N, X_{u u}\right\rangle \\
& f=-\left\langle N_{u}, X_{v}\right\rangle=\left\langle N, X_{u v}\right\rangle=\left\langle N_{v}, X_{u}\right\rangle \\
& g=-\left\langle N_{v}, X_{v}\right\rangle=\left\langle N, X_{v v}\right\rangle
\end{aligned}
$$

e as igualdades são obtidas derivando-se a expressão $\left\langle N, X_{u}\right\rangle=\left\langle N, X_{v}\right\rangle=0$. As funções envolvidas nestas contas foram calculadas no ponto $p$, o qual foi omitido por simplicidade. Fazendo alguns cálculos (ver [5]), obtemos

$$
K=\frac{e g-f^{2}}{E G-F^{2}}, \quad H=\frac{1}{2} \frac{e G-2 f F+g E}{E G-F^{2}},
$$

de onde segue que as curvaturas principais são as soluções da equação

$$
\kappa^{2}-2 H \kappa+K=0
$$

e, portanto,

$$
\kappa=H \pm \sqrt{H^{2}-K}
$$

A seguir, vamos entender um pouco sobre contato de ordem $\geq 2$ entre superfícies, um tópico que pode ser encontrado com mais detalhes em [2], [5] e [15]. Suponha que $S_{1}$ e $S_{2}$ se intersectam tangencialmente no ponto $p_{0}$, isto é, os planos tangentes $T_{p_{0}} S_{1}$ e $T_{p_{0}} S_{2}$ são iguais. Inicialmente, considere a projeção ortogonal $\pi: \mathbb{R}^{3} \rightarrow p_{0}+T_{p_{0}} S_{1}=p_{0}+T_{p_{0}} S_{2}$. Temos que $\pi\left(p_{0}\right)=p_{0}, \pi(p)-p_{0} \in T_{p_{0}} S_{1}$ e $\pi(p)-p \perp T_{p_{0}} S_{1}$. Veja ainda que a restrição de $\pi$ a cada uma das superfícies tem como diferencial em $p_{0}$ a identidade. Logo, pelo Teorema da Função Inversa, existem abertos $U_{1} \subset S_{1}$ e $U_{2} \subset S_{2}$, vizinhanças de $p_{0}$, nos quais $\pi$ é um difeomorfismo sobre sua imagem. Assim, considere $\delta>0$ tal que o conjunto

$$
B_{\delta}\left(p_{0}\right)=\left\{p \in p_{0}+T_{p_{0}} S_{1} ;\left\|p-p_{0}\right\|<\delta\right\}
$$

esteja contido na interseção $\pi\left(U_{1}\right) \cap \pi\left(U_{2}\right)$. Fixada uma base ortonormal $\left\{w_{1}, w_{2}\right\}$ de $T_{p_{0}} S_{1}$, defina $N_{0}=w_{1} \wedge w_{2}$. Denotando $V_{1}=\pi^{-1}\left(B_{\delta}\left(p_{0}\right)\right) \subset U_{1}$ e $U=B_{\delta}\left(p_{0}\right) \subset p_{0}+T_{p_{0}} S_{1}$, observamos que, dado $p \in V_{1}$, existe $w \in U$ tal que $p=\pi^{-1}(w)$. Para este $w$, existe $(u, v) \in$ $B_{\delta}(0) \subset \mathbb{R}^{2}$ tal que $w=p_{0}+u w_{1}+v w_{2}$, ou seja, um ponto $p \in V_{1}$ é unicamente determinado por $(u, v) \in B_{\delta}(0)$ da seguinte maneira:

$$
p=\left(\left.\pi\right|_{V_{1}}\right)^{-1}(w)=\left(\left.\pi\right|_{V_{1}}\right)^{-1}\left(p_{0}+u w_{1}+v w_{2}\right)
$$

Isto nos permite definir a parametrização $X: B_{\delta}(0) \subset \mathbb{R}^{2} \rightarrow V_{1} \subset S_{1}$ tal que

$$
X(u, v)=\left(\left.\pi\right|_{V_{1}}\right)^{-1}\left(p_{0}+u w_{1}+v w_{2}\right)=p_{0}+u w_{1}+v w_{2}+h_{1}(u, v) N_{0}
$$


para alguma função $h_{1}: B_{\delta}(0) \rightarrow \mathbb{R}$. A última igualdade segue pelo fato de que, dado $p \in V_{1}$, então, como $\pi(p)-p$ é paralelo a $N_{0}$ tem-se que que existe $h_{1}(u, v) \in \mathbb{R}$ tal que $\pi(p)-p=$ $-h_{1}(u, v) N_{0}$. Logo,

$$
p=\pi(p)+h_{1}(u, v) N_{0}=p_{0}+u w_{1}+v w_{2}+h_{1}(u, v) N_{0} .
$$

Com um raciocínio análogo, definimos $Y: B_{\delta}(0) \rightarrow V_{2} \subset S_{2}$ por

$$
Y(u, v)=\left(\left.\pi\right|_{V_{2}}\right)^{-1}\left(p_{0}+u w_{1}+v w_{2}\right)=p_{0}+u w_{1}+v w_{2}+h_{2}(u, v) N_{0}, \quad h_{2}: B_{\delta}(0) \rightarrow \mathbb{R} .
$$

Estas parametrizações correspondem a ver $S_{1}$ e $S_{2}$ localmente como o gráfico das funções $h_{1}$ e $h_{2}$.

Definição 1.15. Dizemos que $S_{1}$ e $S_{2}$ têm contato de ordem $\geq 2$ em $p_{0}$ se

$$
\lim _{(u, v) \rightarrow(0,0)} \frac{h_{1}(u, v)-h_{2}(u, v)}{\|(u, v)\|^{2}}=0,
$$

onde $h_{1}$ e $h_{2}$ são dadas como na discussão acima.

Isto significa que, numa vizinhança de $p_{0}$, as alturas $h_{1}$ e $h_{2}$ estão arbitrariamente próximas e os pontos correspondentes $p_{1} \in S_{1}$ e $p_{2} \in S_{2}$ se aproximam rapidamente de $p_{0}$.

Proposição 1.3. Sejam $S_{1}$ e $S_{2}$ superfícies que se intersectam tangencialmente em $p_{0}$ com uma orientação tal que $N^{S_{1}}$ e $N^{S_{2}}$ coincidam em $p_{0}$. Então, são equivalentes:

1. $S_{1}$ e $S_{2}$ têm contato de ordem $\geq 2$ em $p_{0}$.

2. As restrições a $T_{p_{0}} S_{1}=T_{p_{0}} S_{2}$ das segundas formas fundamentais de $S_{1}$ e $S_{2}$ coincidem.

Falaremos ainda sobre superfícies na forma implícita, que são representadas por funções diferenciáveis $f(x, y, z)=0$. Começamos com o conceito de ponto crítico e valor regular.

Definição 1.16. Dizemos que $p \in U$ é um ponto crítico de uma aplicação diferenciável $f: U \subset \mathbb{R}^{n} \rightarrow$ $\mathbb{R}^{m}$, definida no aberto $U$ do $\mathbb{R}^{n}$, se a diferencial $d f_{p}: \mathbb{R}^{n} \rightarrow \mathbb{R}^{m}$ não é uma aplicação sobrejetiva. $A$ imagem $f(p) \in \mathbb{R}^{m}$ de um ponto crítico é chamado um valor crítico de $f$. Um ponto de $\mathbb{R}^{m}$ que não é um valor crítico é chamado um valor regular de $f$.

Note que, se $f: U \subset \mathbb{R}^{3} \rightarrow \mathbb{R}$ é uma função diferenciável, então, dizer que $d f_{p}$ não é uma aplicação sobrejetiva é equivalente a dizer que $f_{x}(p)=f_{y}(p)=f_{z}(p)=0$. Portanto, $r \in f(U)$ é um valor regular de $f: U \subset \mathbb{R}^{3} \rightarrow \mathbb{R}$ se, e somente se, $f_{x}, f_{y}$ e $f_{z}$ não se anulam simultaneamente em todos os pontos da imagem inversa

$$
f^{-1}(r)=\{(x, y, z) \in U ; f(x, y, z)=r\} .
$$

O Teorema da Função Inversa assegura o seguinte resultado (ver [5, p. 69]). 
Proposição 1.4. Seja $f: U \subset \mathbb{R}^{3} \rightarrow \mathbb{R}$ uma função diferenciável. Se $r \in f(U)$ é um valor regular de $f$, então $S=f^{-1}(r)$ é uma superfície regular. Neste caso, dizemos que $S$ está na forma implícita e escrevemos $f(x, y, z)-r=0$.

Finalizamos esta seção com algumas expressões que serão úteis para futuros cálculos. A curva $(x(s), y(s), z(s))$ com restrição $f(x(s), y(s), z(s))=0$ define uma curva sobre a superfície implícita $f(x, y, z)=0$. Derivando $f(x(s), y(s), z(s))=0$ com respeito a $s$, temos que

$$
\begin{aligned}
0= & \frac{d f}{d s}=f_{x} x^{\prime}+f_{y} y^{\prime}+f_{z} z^{\prime} \\
0= & \frac{d^{2} f}{d s^{2}}=f_{x x}\left(x^{\prime}\right)^{2}+f_{y y}\left(y^{\prime}\right)^{2}+f_{z z}\left(z^{\prime}\right)^{2}+2\left(f_{x y} x^{\prime} y^{\prime}+f_{x z} x^{\prime} z^{\prime}+f_{y z} y^{\prime} z^{\prime}\right)+ \\
& f_{x} x^{\prime \prime}+f_{y} y^{\prime \prime}+f_{z} z^{\prime \prime} \\
0= & \frac{d^{3} f}{d s^{3}}=f_{x x x}\left(x^{\prime}\right)^{3}+f_{y y y}\left(y^{\prime}\right)^{3}+f_{z z z}\left(z^{\prime}\right)^{3}+3\left[f_{x x y}\left(x^{\prime}\right)^{2} y^{\prime}+f_{x x z}\left(x^{\prime}\right)^{2} z^{\prime}+\right. \\
& \left.f_{x y y}\left(y^{\prime}\right)^{2} x^{\prime}+f_{y y z}\left(y^{\prime}\right)^{2} z^{\prime}+f_{y z z}\left(z^{\prime}\right)^{2} y^{\prime}+f_{x z z}\left(z^{\prime}\right)^{2} x^{\prime}+2 f_{x y z} x^{\prime} y^{\prime} z^{\prime}\right]+ \\
& 3\left[f_{x x} x^{\prime} x^{\prime \prime}+f_{y y} y^{\prime} y^{\prime \prime}+f_{z z} z^{\prime} z^{\prime \prime}+f_{x y}\left(x^{\prime \prime} y^{\prime}+x^{\prime} y^{\prime \prime}\right)+f_{x z}\left(x^{\prime \prime} z^{\prime}+x^{\prime} z^{\prime \prime}\right)+\right. \\
& \left.f_{y z}\left(y^{\prime \prime} z^{\prime}+y^{\prime} z^{\prime \prime}\right)\right]+f_{x} x^{\prime \prime \prime}+f_{y} y^{\prime \prime \prime}+f_{z} z^{\prime \prime \prime}
\end{aligned}
$$

\subsection{O Espaço de Lorentz-Minkowski $\mathbb{L}^{3}$}

Abordaremos nesta seção o espaço de Lorentz-Minkowski $\mathbb{L}^{3}$, que é o $\mathbb{R}^{3}$ munido de uma métrica que descreveremos a seguir. Tal tópico está divido em três partes que descrevem as propriedades e definições básicas, as curvas e as superfícies neste ambiente, conceitos estes que serão importantes para o estudo das curvas de interseção entre duas superfícies no espaço de Lorentz-Minkowski. Apresentaremos também algumas ideias a respeito das formas bilineares simétricas, as quais serão muito utilizadas no decorrer do texto. Embora o conceito de forma bilinear simétrica seja considerado com o intuito de aplicá-lo no $\mathbb{L}^{3}$, ele será apresentado em um contexto mais geral, isto é, para um espaço vetorial $V$ arbitrário. Utilizamos como base as referências descritas em [6], [9], [10] e [11].

\subsubsection{Propriedades e Definições Básicas}

Definição 1.17. Considere $\mathbb{R}^{3}$ com a métrica $g=\langle,\rangle_{1}$ que associa a cada $u=\left(u_{1}, u_{2}, u_{3}\right), v=$ $\left(v_{1}, v_{2}, v_{3}\right) \in \mathbb{R}^{3}$ o número real

$$
\langle u, v\rangle_{1}=u_{1} v_{1}+u_{2} v_{2}-u_{3} v_{3}
$$

Chamamos o $\mathbb{R}^{3}$ munido da métrica g de espaço de Lorentz-Minkowski e denotaremos $\left(\mathbb{R}^{3}, g\right)=\mathbb{L}^{3}$. 
A métrica $g=\langle,\rangle_{1}$ é chamada métrica de Lorentz-Minkowski (ou métrica de Lorentz). O subscrito $1 \mathrm{em}\langle,\rangle_{1}$ é devido à assinatura de $g$ (a definição de assinatura é dada mais adiante). Com a métrica $g$, podemos ter vetores $v \in \mathbb{L}^{3}$ tais que $\langle v, v\rangle_{1}<0$ ou ainda $\langle v, v\rangle_{1}=0 \mathrm{com}$ $v \neq 0$. Por exemplo, sendo $e_{1}=(1,0,0)$ e $e_{3}=(0,0,1)$, temos que $\left\langle e_{3}, e_{3}\right\rangle_{1}=-1<0$ e $\left\langle e_{1}+e_{3}, e_{1}+e_{3}\right\rangle_{1}=0$.

Definição 1.18. Seja $v \in \mathbb{L}^{3}$. Dizemos que:

1. vé tipo tempo se $\langle v, v\rangle_{1}<0$,

2. vé tipo luz se $\langle v, v\rangle_{1}=0$ ev $\neq 0$,

3. vé tipo espaço se $\langle v, v\rangle_{1}>0$ ou $v=0$.

Um vetor tipo luz é também chamado de vetor nulo. Denotamos por $\mathcal{T}$ o conjunto de todos os vetores tipo tempo, por $\mathcal{C}$ o conjunto de todos os vetores tipo luz ( $\mathcal{C}$ é chamado o cone de luz) e por $\mathcal{E}$ o conjunto de todos os vetores tipo espaço. Assim, temos que

$$
\begin{aligned}
& \mathcal{T}=\left\{(x, y, z) \in \mathbb{L}^{3} ; x^{2}+y^{2}-z^{2}<0\right\} \\
& \mathcal{C}=\left\{(x, y, z) \in \mathbb{L}^{3} ; x^{2}+y^{2}-z^{2}=0\right\}-\{0\} \\
& \mathcal{E}=\left\{(x, y, z) \in \mathbb{L}^{3} ; x^{2}+y^{2}-z^{2}>0\right\} \cup\{0\} .
\end{aligned}
$$

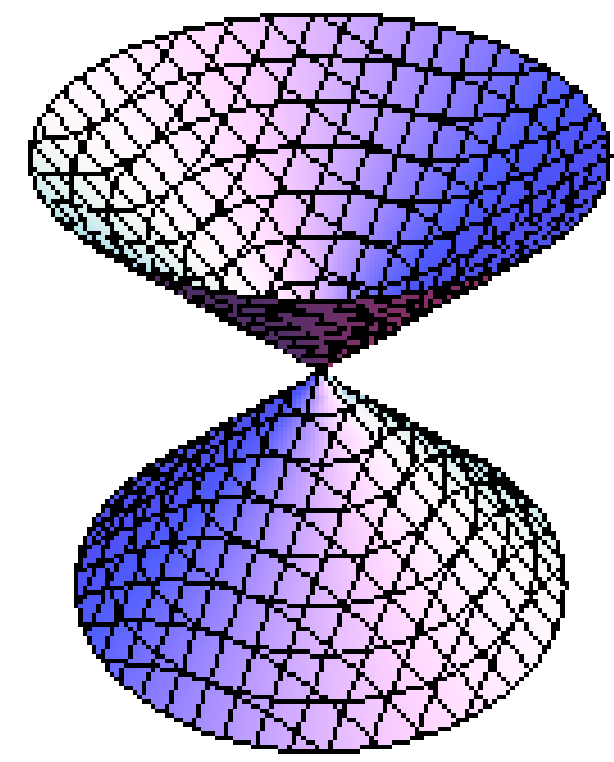

Figura 1.3: O cone de luz 
A figura mostra o cone de luz $\mathcal{C}$. Observe que o interior do cone é o conjunto de todos os vetores tipo tempo $\mathcal{T}$ e o seu exterior (incluindo o zero) é o conjunto $\mathcal{E}$ de todos os vetores tipo espaço.

Uma forma bilinear simétrica em um espaço vetorial $V$ de dimensão finita é uma função bilinear $b: V \times V \rightarrow \mathbb{R}$ que satisfaz $b(v, w)=b(w, v)$, para todo $v, w \in V$. Dizemos que

1. $b$ é positiva (negativa) definida se, dado $v \neq 0$, tem-se $b(v, v)>0(<0)$,

2. $b$ é positiva (negativa) semi-definida se, dado $v \in V$, tem-se $b(v, v) \geq 0(\leq 0)$,

3. $b$ é não degenerada desde que, se $v \in V$ é tal que $b(v, w)=0 \forall w \in V$, então $v=0$.

O índice $v$ de uma forma bilinear simétrica $b$ em um espaço vetorial $V$ é o maior inteiro que é a dimensão de um subespaço $W \subseteq V$ no qual $\left.b\right|_{W}$ é negativa definida. Desta forma, $0 \leq v \leq \operatorname{dim} V$ e $v=0$ se, e somente se, $b$ é positiva semi-definida. É comum nos referirmos ao índice $v$ de uma forma bilinear simétrica não degenerada $b$ de $V$ como o índice de $V$, que é denotado por $v=\operatorname{ind}(V)$.

Se $\mathcal{B}=\left\{e_{1}, \ldots, e_{n}\right\}$ é uma base para $V$, a matriz $\left(b_{i j}\right)=b\left(e_{i}, e_{j}\right)$ é a matriz de b relativa a $\mathcal{B}$.

Lema 1.1. Uma forma bilinear simétrica é não degenerada se, e somente se, sua matriz relativa a uma base (e portanto, a todas) é invertível.

Demonstração. Primeiramente, note que, dada uma base $\mathcal{B}=\left\{e_{1}, \ldots, e_{n}\right\}$ de $V$ então, fixado $v \in V$, tem-se que $b(v, w)=0, \forall w \in V$ se, e somente se, $b\left(v, e_{i}\right)=0$ para $i=1, \ldots, n$. Além disso,

$$
b\left(v, e_{i}\right)=b\left(\sum_{j=1}^{n} v_{j} e_{j}, e_{i}\right)=\sum_{j=1}^{n} v_{j} b_{i j},
$$

para todo $i=1, \ldots, n$. Portanto, $b$ é degenerada se, e somente se, existem números reais, não todos nulos, $v_{1}, \ldots, v_{n}$, tais que $\sum_{j=1}^{n} b_{i j} v_{j}=0$. Como cada $i$ fixado determina um vetor linha da matriz $\left(b_{i j}\right)$, esta condição equivale à dependência linear das linhas de $\left(b_{i j}\right)$, isto é, à matriz $b_{i j}$ não ser invertível. Isto prova o resultado.

Definição 1.19. Dado um subespaço $U \subseteq \mathbb{L}^{3}$, dizemos que $U$ é tipo tempo (resp. tipo luz, tipo espaço) se a métrica de Lorentz-Minkowski induzida em U é não degenerada de índice 1 (resp. degenerada, positiva definida).

O caráter de causalidade de um subespaço $U$ é a propriedade dele ser tipo tempo, tipo luz ou tipo espaço.

Exemplo 1.1. O exemplo dado imediatamente antes da Definição 1.18 mostra um vetor tipo luz que éa soma de dois vetores tipo espaço. Vejamos abaixo alguns exemplos para subespaços. 
- O plano Span $\left\{e_{1}, e_{2}\right\}$ é tipo espaço. De fato, dado $v \in \operatorname{Span}\left\{e_{1}, e_{2}\right\}$ não nulo, existem $\lambda, \mu \in \mathbb{R}$ tais que $v=\lambda e_{1}+\mu e_{2}$. Como $v$ é não nulo, pelo menos um dos coeficientes $\lambda, \mu$ é diferente de zero. $\log o$,

$$
\langle v, v\rangle_{1}=\left\langle\lambda e_{1}+\mu e_{2}, \lambda e_{1}+\mu e_{2}\right\rangle_{1}=\lambda^{2}\left\langle e_{1}, e_{1}\right\rangle_{1}+2 \lambda \mu\left\langle e_{1}, e_{2}\right\rangle_{1}+\mu^{2}\left\langle e_{2}, e_{2}\right\rangle_{1}>0,
$$

ou seja, $\left.g\right|_{\text {Span }\left\{e_{1}, e_{2}\right\}}$ é positiva definida. Na verdade, observe que qualquer subespaço gerado por dois vetores tipo espaço que são ortogonais é ainda tipo espaço.

- Os planos Span $\left\{e_{1}, e_{3}\right\}$ e Span $\left\{e_{2}, e_{3}\right\}$ são tipo tempo. Vamos mostrar o primeiro caso. Suponha que $v \in \operatorname{Span}\left\{e_{1}, e_{3}\right\}$ é tal que $\langle v, w\rangle_{1}=0$ para todo $w \in \operatorname{Span}\left\{e_{1}, e_{3}\right\}$ e escreva $v=a_{1} e_{1}+$ $a_{3} e_{3}$ e $w=b_{1} e_{1}+b_{3} e_{3}$. Então

$$
0=\langle v, w\rangle_{1}=a_{1} a_{3}\left\langle e_{1}, e_{1}\right\rangle_{1}+\left(a_{1} b_{3}+a_{3} b_{1}\right)\left\langle e_{1}, e_{3}\right\rangle_{1}+a_{3} b_{3}\left\langle e_{3}, e_{3}\right\rangle_{1}=a_{1} b_{1}-a_{3} b_{3}
$$

Como esta igualdade vale para todo $w \in \operatorname{Span}\left\{e_{1}, e_{3}\right\}$, vale em particular para $w=e_{1}$, isto é, $b_{1}=1, b_{3}=0$, o que implica $a_{1}=0$. Por outro lado, tomando $w=e_{3}$, temos $b_{1}=0, b_{3}=1$, que implica $a_{3}=0$. Portanto, $v=0$. Precisamos ainda ver que o indice $v$ de $\left.g\right|_{\text {span }\left\{e_{1}, e_{3}\right\}} e$ igual a 1. De fato, $\left.g\right|_{S p a n}\left\{e_{1}, e_{3}\right\}$ não é negativa definida e qualquer subespaço próprio não nulo tem dimensão 1. Como existem vetores tipo tempo em $\left\{e_{1}, e_{3}\right\}$, concluímos que o maior inteiro que é a dimensão de um subespaço $W \subseteq\left\{e_{1}, e_{3}\right\}$ no qual $\left.b\right|_{W}$ é negativa definida é 1 . Portanto, Span $\left\{e_{1}, e_{3}\right\}$ é tipo tempo.

- O plano Span $\left\{e_{1}, e_{2}+e_{3}\right\}$ é tipo luz. Para ver isto, precisamos mostrar que a métrica de LorentzMinkowski restrita a Span $\left\{e_{1}, e_{2}+e_{3}\right\}$ é degenerada. Dado $w \in \operatorname{Span}\left\{e_{1}, e_{2}+e_{3}\right\}$, existem $a, b \in \mathbb{R}$ tais que $w=a e_{1}+b\left(e_{2}+e_{3}\right)=(a, 0,0)+(0, b, b)=(a, b, b)$. Tome $c \neq 0 e$ $v=(0, c, c)=c\left(e_{2}+e_{3}\right) \in \operatorname{Span}\left\{e_{1}, e_{2}+e_{3}\right\} . \log o$,

$$
\langle v, w\rangle_{1}=\left\langle c\left(e_{2}+e_{3}\right), a e_{1}+b\left(e_{2}+e_{3}\right)\right\rangle_{1}=b c\left(\left\langle e_{2}, e_{2}\right\rangle_{1}+2\left\langle e_{2}, e_{3}\right\rangle_{1}+\left\langle e_{3}, e_{3}\right\rangle_{1}\right)=0 .
$$

Portanto, $\left.g\right|_{\operatorname{Span}\left\{e_{1}, e_{2}+e_{3}\right\}}$ é degenerada, como queríamos demonstrar.

Definição 1.20. Sejam $v, w \in \mathbb{L}^{3} e U \subseteq \mathbb{L}^{3}$.

1. A norma ou o comprimento de vé definido por $\|v\|_{1}=\sqrt{\left|\langle v, v\rangle_{1}\right|}$.

2. Dizemos que vé unitário se $\|v\|_{1}=1$.

3. Os vetores $v, w$ são ortogonais se $\langle v, w\rangle_{1}=0$.

4. O subespaço ortogonal de $U$ é o conjunto $U^{\perp}$ dado por $U^{\perp}=\left\{v \in \mathbb{L}^{3} ;\langle u, v\rangle_{1}=0, \forall u \in U\right\}$.

5. Dizemos que U é não degenerado se a métrica de Lorentz-Minkowski restrita a U é não degenerada. 
Lema 1.2. Seja U um subespaço de um espaço não degenerado $V$. Então

1. $\operatorname{dim}\left(U^{\perp}\right)=\operatorname{dim} V-\operatorname{dim} U$,

2. $\left(U^{\perp}\right)^{\perp}=U$.

Demonstração. $\quad$ 1. Dado $w \in U^{\perp}$, tem-se que

$$
\langle u, w\rangle_{1}=0, \forall u \in U
$$

Seja $m=\operatorname{dim} U$ e $\mathcal{B}=\left\{e_{1}, \ldots, e_{m}\right\}$ uma base de $U$. Podemos completar $\mathcal{B}$ para formar uma base de $V$. Assim, seja $\left\{e_{1}, \ldots, e_{m}, \ldots, e_{n}\right\}$ base de $V$. Pela equação (1.12), escrevendo $w=\sum_{j=1}^{n} a_{j} e_{j}$, como para cada $i=1, \ldots, m, e_{i} \in U$, segue que $\left\langle e_{i}, w\right\rangle_{1}=0$. Logo, para todo $i=1, \ldots, m$,

$$
0=\left\langle e_{i}, \sum_{j=1}^{n} a_{j} e_{j}\right\rangle_{1}=\sum_{j=1}^{n} a_{j}\left\langle e_{i}, e_{j}\right\rangle_{1},
$$

isto é, denotando $g_{i j}=\left\langle e_{i}, e_{j}\right\rangle_{1}$,

$$
\sum_{j=1}^{n} a_{j} g_{i j}=0
$$

Como $i \in\{1, \ldots, m\}$, a equação (1.12) nos dá um sistema de $m$ equações lineares com $n$ incógnitas $a_{1}, \ldots, a_{n}$. Sendo $g=\langle,\rangle_{1}$ não degenerada, a matriz dos coeficientes de $g$ é invertível, ou seja, é invertível a matriz

$$
M=\left[\begin{array}{ccc}
g_{11} & \ldots & g_{1 n} \\
\vdots & \ddots & \vdots \\
g_{n 1} & \ldots & g_{n n}
\end{array}\right]
$$

Assim, segue da Álgebra Linear que o espaço das soluções de (1.13) tem dimensão $n-m$. Mas, por construção, as soluções $\left(a_{1}, \ldots, a_{n}\right)$ dão exatamente os vetores $w=\sum_{j=1}^{n} a_{j} e_{j} \in$ $U^{\perp}$. Portanto, $\operatorname{dim}\left(U^{\perp}\right)=n-m=\operatorname{dim} V-\operatorname{dim} U$.

2. Dado $u \in U$, então, para todo $w \in U^{\perp}$, tem-se $\langle u, w\rangle_{1}=0$. Logo, $u \in\left(U^{\perp}\right)^{\perp}$ e, portanto, $U \subset\left(U^{\perp}\right)^{\perp}$. Além disso, pelo item 1 ,

$$
\operatorname{dim}\left(U^{\perp}\right)^{\perp}=\operatorname{dim} V-\operatorname{dim}\left(U^{\perp}\right)=\operatorname{dim} V-(\operatorname{dim} V-\operatorname{dim} U)=\operatorname{dim} U .
$$

Assim, $U=\left(U^{\perp}\right)^{\perp}$. 
Lema 1.3. Se $V \neq 0$ é um espaço n-dimensional munido de uma forma bilinear simétrica não degenerada, $b$, então $V$ admite uma base ortonormal.

Demonstração. Para ver isso, observe que, como $V$ é não degenerado, então existe $v \in V$ tal que $b(v, v) \neq 0$. Provaremos o resultado por indução sobre $n=\operatorname{dim} V$. Se $n=1$, seja $v \in V$ tal que $b(v, v) \neq 0$. Então, $w=\frac{v}{\|v\|}$ é unitário e a base $\{w\}$ é ortonormal. Suponha que o resultado é válido para todo $k<n$. Considerando o subespaço $\operatorname{Span}\{v\}^{\perp}$, temos pelo item 2 do Lema 1.2 que $\operatorname{dim}\left(\operatorname{Span}\{v\}^{\perp}\right)=n-1$. Assim, por hipótese de indução, existe $\left\{e_{1}, \ldots, e_{n-1}\right\}$ base ortonormal de $\operatorname{Span}\{v\}^{\perp}$, Note que $v \perp e_{i}, \forall i=1, \ldots, n-1$ e, como $b$ é não degenerada, $v$ e $e_{i}$ são linearmente independentes. Sendo $b(v, v) \neq 0$, podemos considerar o vetor unitário $w=\frac{v}{\|v\|}$. Portanto, $\left\{e_{1}, \ldots, e_{n-1}, w\right\}$ é base ortonormal de $V$.

Considere uma base ortonormal $\left\{e_{1}, \ldots, e_{n}\right\}$ do espaço vetorial $V$ munido da forma bilinear simétrica não degenerada $b$. Dados $v, w \in V$ escreva $v=v_{1} e_{1}+\ldots+v_{n} e_{n}$ e $w=w_{1} e_{1}+\ldots+$ $w_{n} e_{n}$. Então,

$$
b(v, w)=v_{1} w_{1} b\left(e_{1}, e_{1}\right)+v_{2} w_{2} b\left(e_{2}, e_{2}\right)+\ldots+v_{n} w_{n} b\left(e_{n}, e_{n}\right) .
$$

Note que, como cada $e_{i}$ é unitário, então $b\left(e_{i}, e_{i}\right)= \pm 1$. Assim, a expressão de $b(v, w)$ é

$$
b(v, w)=v_{1} w_{1} \pm v_{2} w_{2} \pm \ldots \pm v_{n} w_{n}
$$

ou, na forma matricial,

$$
b(v, w)=\left[\begin{array}{lll}
v_{1} & \ldots & v_{n}
\end{array}\right]\left[\begin{array}{rrrr} 
\pm 1 & 0 & \ldots & 0 \\
0 & \pm 1 & \ldots & 0 \\
\vdots & \vdots & & \vdots \\
0 & 0 & \ldots & \pm 1
\end{array}\right]\left[\begin{array}{c}
w_{1} \\
\vdots \\
w_{n}
\end{array}\right]=v^{T}\left[\begin{array}{rrrr} 
\pm 1 & 0 & \ldots & 0 \\
0 & \pm 1 & \ldots & 0 \\
\vdots & \vdots & & \vdots \\
0 & 0 & \ldots & \pm 1
\end{array}\right] w .
$$

Ou seja, a matriz $B$ de $b$ na base ortonormal $\left\{e_{1}, \ldots, e_{n}\right\}$ é diagonal com coeficientes \pm 1 . Logo, seu determinante é igual a \pm 1 e $B$ é invertível. A assinatura de $b$ indica a posição e o número de sinais negativos na matriz de $b$ e é denotada por $\left(\varepsilon_{1}, \ldots, \varepsilon_{n}\right)$, onde $\varepsilon_{j}=b\left(e_{j}, e_{j}\right)= \pm 1$. Convencionaremos escrever os sinais negativos (quando houver) nas últimas entradas da assinatura. Por exemplo, se $V=\mathbb{L}^{3}$, então

$$
B=\left[\begin{array}{rrr}
1 & 0 & 0 \\
0 & 1 & 0 \\
0 & 0 & -1
\end{array}\right]
$$

e a assinatura da métrica $g$ é $(+,+,-)$, em que $g=\langle,\rangle_{1}$.

Proposição 1.5. Para toda base ortonormal $\left\{e_{1}, \ldots, e_{n}\right\}$ de $V$, o número de sinais negativos na assinatura $\left(\varepsilon_{1}, \ldots, \varepsilon_{n}\right)$ é o índice v de $V$. 
A demonstração deste resultado pode ser vista em [11, p. 51].

Dada uma forma bilinear em um espaço vetorial, podemos falar em ortogonalidade entre vetores.

Definição 1.21. Dizemos que os vetores $u, v$ no espaço vetorial $V$ munido de uma forma bilinear simétrica $b$ são ortogonais se $b(u, v)=0$.

Proposição 1.6. Um subespaço $U$ de $V$ é não degenerado se, e somente se, $V=U \oplus U^{\perp}$.

Demonstração. Sabe-se, por um resultado da Álgebra Linear, que

$$
\operatorname{dim}\left(U+U^{\perp}\right)+\operatorname{dim}\left(U \cap U^{\perp}\right)=\operatorname{dim} U+\operatorname{dim}\left(U^{\perp}\right) .
$$

Pelo item 1 do Lema 1.2, $\operatorname{dim} U+\operatorname{dim}\left(U^{\perp}\right)=\operatorname{dim} V$. Logo, $V=U \oplus U^{\perp}$ se, e somente se, $\operatorname{dim}\left(U \cap U^{\perp}\right)=0$. Ora, sendo $U \cap U^{\perp}=\{u \in U ; u \perp U\}$, então $U \cap U^{\perp}=0$ é equivalente a $\left.b\right|_{u}$ ser não degenerada, ou seja, a $U$ ser não degenerado. Daí, segue o resultado.

Concluímos que, como $U=\left(U^{\perp}\right)^{\perp}$, então $U$ é não degenerado se, e somente se $V=$ $\left(U^{\perp}\right)^{\perp} \oplus U^{\perp}$, que equivale a $U^{\perp}$ ser não degenerado.

Proposição 1.7. Seja $v \in \mathbb{L}^{3} e U \subseteq \mathbb{L}^{3}$ um subespaço.

1. vé tipo tempo se, e somente se, $\operatorname{Span}\{v\}^{\perp}$ é tipo espaço.

2. $v \neq 0$ é tipo espaço se, e somente se, $\operatorname{Span}\{v\}^{\perp}$ é tipo tempo.

3. U é tipo tempo se, e somente se, $U^{\perp}$ é tipo espaço.

4. U é tipo luz se, e somente se, $U^{\perp}$ é tipo luz.

\section{Demonstração.}

1. Sem perda de generalidade, suponha que $v$ é unitário. Pelo Lema $1.2, \operatorname{dim}\left(\operatorname{Span}\{v\}^{\perp}\right)=$ $\operatorname{dim} \mathbb{L}^{3}-\operatorname{dim} \operatorname{Span}\{v\}=3-1=2$. Sendo $v$ tipo tempo então $\operatorname{Span}\{v\}^{\perp}$ é não degenerado. Assim, podemos considerar $\left\{v_{1}, v_{2}\right\}$ base ortonormal de $\operatorname{Span}\{v\}^{\perp}$. Note que, para $i=1,2$, $v$ e $v_{i}$ são L.I. pois, caso contrário, existiria $\lambda \neq 0$ tal que $v=\lambda v_{i}$ e, então,

$$
0=\left\langle v, v_{i}\right\rangle_{1}=\lambda\left\langle v_{i}, v_{i}\right\rangle_{1} \neq 0
$$

Portanto, $\left\{v_{1}, v_{2}, v\right\}$ é base ortonormal de $\mathbb{L}^{3}$. Como $v$ é tipo tempo e o índice de $\mathbb{L}^{3}$ é $v=1$, então $v_{1}$ e $v_{2}$ são tipo espaço. Portanto, $\operatorname{Span}\{v\}^{\perp}=\operatorname{Span}\left\{v_{1}, v_{2}\right\}$ é tipo espaço.

Agora, suponha que $\operatorname{Span}\{v\}^{\perp}$ seja tipo espaço. Considerando $\left\{v_{1}, v_{2}\right\}$ base ortonormal de $\operatorname{Span}\{v\}^{\perp}$, temos que $\left\{v_{1}, v_{2}, v\right\}$ forma uma base de $\mathbb{L}^{3}$. Como $v_{1}, v_{2}$ são tipo espaço, então $v$ é tipo tempo. 
2. Suponha que $v$ é unitário. Sendo $v$ tipo espaço então $\operatorname{Span}\{v\}$ é não degenerado e, portanto, $\operatorname{Span}\{v\}^{\perp}$ é não degenerado. Seja $\left\{v_{1}, v_{2}\right\}$ base ortonormal de $\operatorname{Span}\{v\}^{\perp}$. Então, $\left\{v_{1}, v_{2}, v\right\}$ é base ortonormal de $\mathbb{L}^{3}$. Analogamente ao que fizemos na demonstração do item anterior, temos que, como $v$ é tipo espaço, então ou $v_{1}$ é tipo tempo e $v_{2}$ é tipo espaço, ou vice-versa. Portanto, $\operatorname{Span}\{v\}^{\perp}$ é não degenerado de índice 1, isto é, $\operatorname{Span}\{v\}^{\perp}$ é tipo tempo.

Reciprocamente, se $\operatorname{Span}\{v\}^{\perp}$ é tipo tempo, considere uma base ortonormal $\left\{v_{1}, v_{2}\right\}$ de $\operatorname{Span}\{v\}^{\perp}$ tal que $v_{1}$ é tipo espaço e $v_{2}$ é tipo tempo. Ora, sendo $\operatorname{Span}\{v\}^{\perp}$ não degenerado, então $\operatorname{Span}\{v\}$ é também não degenerado e, assim, $v$ é linearmente independente $\operatorname{com} v_{1}$ e $v_{2}$. Logo, $\left\{v_{1}, v_{2}, v\right\}$ é uma base ortonormal de $\mathbb{L}^{3}$ e, portanto, $v$ é tipo espaço.

3. $U$ tipo tempo implica $U$ e $U^{\perp}$ não degenerados. Portanto, podemos tomar bases ortonormais $\mathcal{B}_{1}$ de $U$ e $\mathcal{B}_{2}$ de $U^{\perp}$. Como $\mathbb{L}^{2}=U \oplus U^{\perp}$ (ver Proposição 1.6), segue que $\mathcal{B}_{1} \cup \mathcal{B}_{2}$ é base ortonormal de $\mathbb{L}^{3}$. Assim,

$$
\operatorname{ind}\left(\mathbb{L}^{3}\right)=\operatorname{ind}(U)+\operatorname{ind}\left(U^{\perp}\right) \Rightarrow 1=1+\operatorname{ind}\left(U^{\perp}\right) \Rightarrow \operatorname{ind}\left(U^{\perp}\right)=0 .
$$

Logo, $U^{\perp}$ é tipo espaço.

Reciprocamente (e analogamente), se $U^{\perp}$ é tipo espaço, então ind $\left(U^{\perp}\right)=0$, que implica $\operatorname{ind}(U)=1 \mathrm{e}$, assim, $U$ é tipo tempo.

4. Seja $U$ tipo luz. Se $U^{\perp}$ não fosse tipo luz, então $U^{\perp}$ seria tipo tempo ou tipo espaço, o que, pelo item anterior, implicaria que $U$ é tipo espaço ou tipo tempo, o que é uma contradição. Logo, $U^{\perp}$ é tipo luz. A volta é inteiramente análoga.

Proposição 1.8. Se u e v são dois vetores tipo luz, então eles são L.D. se, e somente se, são ortogonais.

Demonstração. A ida é imediata. Provaremos a volta do resultado. Seja $e_{3}=(0,0,1) \in \mathbb{L}^{3}$ tipo tempo. Então, $\operatorname{Span}\left\{e_{3}\right\}^{\perp}$ é tipo espaço e, portanto, não degenerado. Logo

$$
\mathbb{L}^{3}=\operatorname{Span}\left\{e_{3}\right\} \oplus \operatorname{Span}\left\{e_{3}\right\}^{\perp} .
$$

Considere $u, v$ em $\mathbb{L}^{3}$ tipo luz e ortogonais, isto é, $\langle u, v\rangle_{1}=0$. Existem, por (1.15), $\alpha, \beta \in \mathbb{R}$ e $\tilde{u}, \tilde{v} \in \operatorname{Span}\left\{e_{3}\right\}^{\perp}$ tais que

$$
\begin{aligned}
& u=\alpha e_{3}+\tilde{u} \\
& v=\beta e_{3}+\tilde{v} .
\end{aligned}
$$

Temos o seguinte:

$$
0=\langle u, u\rangle_{1}=\left\langle\alpha e_{3}+\tilde{u}, \alpha e_{3}+\tilde{u}\right\rangle_{1}=-\alpha^{2}+\langle\tilde{u}, \tilde{u}\rangle_{1} .
$$


Logo, $\alpha= \pm\|\tilde{u}\|_{1}$. Analogamente, obtemos $\beta= \pm\|\tilde{v}\|_{1}$.

Suponha que $\alpha$ e $\beta$ tenham o mesmo sinal. Assim, $\alpha \beta=\|\tilde{u}\|_{1}\|\tilde{v}\|_{1}$ e, então,

$$
0=\langle u, v\rangle_{1}=\left\langle\alpha e_{3}+\tilde{u}, \beta e_{3}+\tilde{v}\right\rangle_{1}=-\alpha \beta+\langle\tilde{u}, \tilde{v}\rangle_{1}=-\|\tilde{u}\|_{1}\|\tilde{v}\|_{1}+\langle\tilde{u}, \tilde{v}\rangle_{1} .
$$

Logo,

$$
\langle\tilde{u}, \tilde{v}\rangle_{1}=\|\tilde{u}\|_{1}\|\tilde{v}\|_{1}
$$

Ora, $\operatorname{Span}\left\{e_{3}\right\}^{\perp}$ é tipo espaço. Logo, $\left.g\right|_{\operatorname{Span}\left\{e_{3}\right\}^{\perp}}$ é positiva definida e, portanto, vale a desigualdade de Cauchy-Schwarz a qual, aplicada a (1.17), nos diz que $\tilde{u}$ e $\tilde{v}$ são L.D., ou seja, existe $\lambda \in \mathbb{R}$ tal que

$$
\tilde{u}=\lambda \tilde{v}
$$

Daí,

$$
u=\alpha e_{3}+\tilde{u}= \pm\|\tilde{u}\|_{1} e_{3}+\tilde{u}= \pm|\lambda|\|\tilde{v}\|_{1} e_{3}+\lambda \tilde{v}= \pm|\lambda|( \pm \beta) e_{3}+\lambda \tilde{v}=|\lambda| \beta e_{3}+\lambda \tilde{v} .
$$

Note que $\lambda \geq 0$. De fato, pelas equações (1.17) e (1.18) e do fato de $\tilde{u}$ e $\tilde{v}$ serem tipo espaço,

$$
\lambda=\frac{\langle\tilde{u}, \tilde{v}\rangle_{1}}{\langle\tilde{v}, \tilde{v}\rangle_{1}}=\frac{\|\tilde{u}\|_{1}\|\tilde{v}\|_{1}}{\langle\tilde{v}, \tilde{v}\rangle_{1}}>0
$$

Portanto, $|\lambda|=\lambda$. Substituindo em (1.19), temos $u=\lambda\left(\beta e_{3}+\tilde{v}\right)=\lambda v$, ou seja, $u$ e $v$ são linearmente dependentes.

Se, porém, $\alpha$ e $\beta$ têm sinais contrários, concluímos que

$$
-\langle\tilde{u}, \tilde{v}\rangle_{1}=\|\tilde{u}\|_{1}\|\tilde{v}\|_{1}
$$

Como $\|\tilde{u}\|_{1}=\|-\tilde{u}\|_{1}$, segue que

$$
\langle-\tilde{u}, \tilde{v}\rangle_{1}=\|-\tilde{u}\|_{1}\|\tilde{v}\|_{1}
$$

e, como - ̃u é também tipo espaço, aplicando Cauchy-Schwarz (assim como antes), tem-se que - $\tilde{u}$ e $\tilde{v}$ são L.D., de onde segue que $u$ e $v$ são L.D., como queríamos demonstrar.

Proposição 1.9. Seja $U \subseteq \mathbb{L}^{3}$ um subespaço bidimensional. As seguintes afirmações são equivalentes.

1. U é tipo tempo.

2. U contém dois vetores tipo luz linearmente independentes.

3. U contém um vetor tipo tempo.

Demonstração. 
$(1) \Rightarrow(2)$. Suponha que $U$ é tipo tempo. Então $U$ é não degenerado de índice 1 e, portanto, existe uma base ortonormal $\left\{e_{1}, e_{2}\right\}$ de $U$ tal que $e_{1}$ é tipo espaço e $e_{2}$ é tipo tempo. Assim, os vetores $e_{1}+e_{2}$ e $e_{1}-e_{2}$ são tipo luz, pois

$$
\left\langle e_{1} \pm e_{2}, e_{1} \pm e_{2}\right\rangle_{1}=\left\langle e_{1}, e_{1}\right\rangle_{1} \pm 2\left\langle e_{1}, e_{2}\right\rangle_{1}+\left\langle e_{2}, e_{2}\right\rangle_{1}=1-1=0 .
$$

Além disso, estes vetores são linearmente independentes, pois $\left\langle e_{1}+e_{2}, e_{1}-e_{2}\right\rangle_{1}=2 \neq 0$. Logo, pela Proposição 1.8, segue.

(2) $\Rightarrow(3)$. Sejam $u, v \in U$ linearmente independentes e tipo luz. Então, pela Proposição 1.8, $\langle u, v\rangle_{1} \neq 0$. Além disso, $\langle u, u\rangle_{1}=\langle v, v\rangle_{1}=0$. Note que

$$
\begin{aligned}
& \langle u+v, u+v\rangle_{1}=2\langle u, v\rangle_{1} \neq 0 \\
& \langle u-v, u-v\rangle_{1}=-2\langle u, v\rangle_{1} \neq 0 .
\end{aligned}
$$

Do fato de $\langle u, v\rangle_{1} \neq 0$, temos que ou $\langle u, v\rangle_{1}>0$ ou $\langle u, v\rangle_{1}<0$. Se for $\langle u, v\rangle_{1}>0$, então $\langle u-v, u-v\rangle_{1}=-2\langle u, v\rangle_{1}<0$. Por outro lado, se for $\langle u, v\rangle_{1}<0$, concluímos que $\langle u+v, u+v\rangle_{1}=2\langle u, v\rangle_{1}<0$. Ou seja, sempre existe um vetor tipo tempo em $U$.

(3) $\Rightarrow(1)$. Seja $u \in U$ um vetor tipo tempo. Então, $\operatorname{Span}\{u\}^{\perp}$ é tipo espaço. Dado $v \in U^{\perp}$, temos em particular que $v \perp u$. Assim, $v \in \operatorname{Span}\{u\}^{\perp}$. Logo, $U^{\perp} \subset \operatorname{Span}\{u\}^{\perp}$ e, assim, $U^{\perp}$ é também tipo espaço. Logo, pelo item 3 da Proposição 1.7, segue que $U$ é tipo tempo.

Proposição 1.10. Seja $U \subseteq \mathbb{L}^{3}$ um subespaço. As seguintes afirmações são equivalentes.

1. Ué tipo luz.

2. U contém um vetor tipo luz, mas nenhum vetor tipo tempo.

3. $U \cap \mathcal{C}=\mathcal{L}-\{0\}$, onde $\mathcal{L}$ é um subespaço de dimensão 1 e $\mathcal{C}$ é o cone de luz.

Demonstração. Note que, se $\operatorname{dim} U=1$, o resultado segue. Vejamos para $\operatorname{dim} U=2$.

$(1) \Rightarrow(2)$. $U$ tipo luz implica que existe $v \in U$ não nulo tal que $\langle v, u\rangle_{1}=0$, para todo $u \in U$. Em particular, $\langle v, v\rangle_{1}=0$. Assim, existe $v \in U$ tipo luz. E, pela proposição anterior, segue que $U$ não contém vetores tipo tempo.

$(2) \Rightarrow(3)$. Como $U$ contém um vetor tipo luz, então $U \cap \mathcal{C}$ é não vazio. Escreva $U \cap \mathcal{C}=\mathcal{L}-$ $\{0\}$, onde $\mathcal{L}$ é um subespaço de $\mathbb{L}^{3}$. Se existissem dois vetores linearmente independentes tipo luz em $\mathcal{L}$, estes gerariam um subespaço de $\mathbb{L}^{3}$ de dimensão dois que, pela proposição anterior, conteria um vetor tipo tempo, gerando uma contradição. Logo, $\operatorname{dim} \mathcal{L}=1$. 
(3) $\Rightarrow(1)$. Sendo $U \cap \mathcal{C}=\mathcal{L}-\{0\} \neq \varnothing, U$ não pode ser tipo espaço. Além disso, pela proposição anterior, $U$ não pode ser tipo tempo, já que $U$ não contém dois vetores L.I. tipo luz. Portanto, $U$ é tipo luz.

Definição 1.22. Se ué um vetor tipo tempo, definimos o cone tipo tempo de u pelo conjunto

$$
C(u)=\left\{v \in \mathcal{T} ;\langle u, v\rangle_{1}<0\right\} .
$$

O cone tipo tempo oposto é

$$
C(-u)=-C(u)=\left\{v \in \mathcal{T} ;\langle u, v\rangle_{1}>0\right\} .
$$

Estes conjuntos são não vazios, visto que $u \in C(u) \mathrm{e}-u \in-C(u)$.

Lema 1.4. Dois vetores $v, w \in \mathbb{L}^{3}$ tipo tempo estão no mesmo cone tipo tempo se, e somente se, $\langle v, w\rangle_{1}<0$.

Demonstração. É claro que, se $\langle v, w\rangle_{1}<0$, então $v$ e $w$ estão no mesmo cone tipo tempo. Reciprocamente, supondo que $v, w \in C(u)$, para algum vetor $u$ tipo tempo, temos que $\langle u, v\rangle_{1}<0$ e $\langle u, w\rangle_{1}<0$. Sem perda de generalidade, suponha que $u$ é unitário. Sendo $\mathbb{L}^{3}=\operatorname{Span}\{u\} \oplus$ $\operatorname{Span}\{u\}^{\perp}$, podemos escrever

$$
v=\lambda u+\tilde{v}, \quad w=\mu u+\tilde{w}, \quad \tilde{v}, \tilde{w} \in \operatorname{Span}\{u\}^{\perp} .
$$

Logo, como $v$ e $w$ são tipo tempo e $u$ é unitário, então, analisando os produtos $\langle v, v\rangle_{1}$ e $\langle w, w\rangle_{1}$, segue que

$$
\|\tilde{v}\|_{1}<|\lambda|, \quad\|\tilde{w}\|_{1}<|\mu|
$$

Além disso,

$$
\langle v, w\rangle_{1}=-\lambda \mu+\langle\tilde{v}, \tilde{w}\rangle_{1} .
$$

Pela desigualdade de Cauchy (lembre que $\tilde{v}$ e $\tilde{w}$ são tipo espaço),

$$
\langle\tilde{v}, \tilde{w}\rangle_{1} \leq\|\tilde{v}\|_{1}\|\tilde{w}\|_{1}<|\lambda \| \mu| .
$$

Ora, $\langle u, v\rangle_{1}=\lambda\langle u, u\rangle_{1}+\langle u, \tilde{v}\rangle_{1}=-\lambda$. Logo, como $\langle u, v\rangle_{1}<0$, temos que $\lambda>0$ e, analogamente, segue que $\mu>0$. Portanto, $\langle\tilde{v}, \tilde{w}\rangle_{1}<\lambda \mu \mathrm{e}$, assim, pela equação (1.21),

$$
\langle v, w\rangle_{1}<-\lambda \mu+\lambda \mu=0,
$$

como queríamos demonstrar. 
Para vetores tipo tempo, vale a desigualdade reversa de Cauchy:

Proposição 1.11 (Desigualdade reversa de Cauchy). Sejam v e w vetores tipo tempo. Então,

$$
\left|\langle v, w\rangle_{1}\right| \geq\|v\|_{1}\|w\|_{1}
$$

e a igualdade vale se, e somente se, os vetores são proporcionais.

Demonstração. Como $\mathbb{L}^{3}=\operatorname{Span}\{v\} \oplus \operatorname{Span}\{v\}^{\perp}$, podemos escrever, para algum $\lambda \in \mathbb{R}$,

$$
w=\lambda v+\tilde{w}, \quad \tilde{w} \in \operatorname{Span}\{v\}^{\perp} .
$$

Logo,

$$
\langle w, w\rangle_{1}=\lambda^{2}\langle v, v\rangle_{1}+\langle\tilde{w}, \tilde{w}\rangle_{1} \Rightarrow \lambda^{2}\langle v, v\rangle_{1}=\langle w, w\rangle_{1}-\langle\tilde{w}, \tilde{w}\rangle_{1}
$$

Portanto,

$$
\langle v, w\rangle_{1}^{2}=\lambda^{2}\langle v, v\rangle_{1}^{2}=\langle v, v\rangle_{1}\left(\langle w, w\rangle_{1}-\langle\tilde{w}, \tilde{w}\rangle_{1}\right) \geq\langle v, v\rangle_{1}\langle w, w\rangle_{1}
$$

já que $\langle v, v\rangle_{1}<0$ e $\langle\tilde{w}, \tilde{w}\rangle_{1} \geq 0$. Daí, segue a desigualdade.

Além disso, como $\langle v, w\rangle_{1}^{2}=\langle v, v\rangle_{1}\left(\langle w, w\rangle_{1}-\langle\tilde{w}, \tilde{w}\rangle_{1}\right)$, então $\langle v, w\rangle_{1}^{2}=\langle v, v\rangle_{1}\langle w, w\rangle_{1}$ se, e somente se, $\langle\tilde{w}, \tilde{w}\rangle_{1}=0$, que equivale a $\tilde{w}=0$, ou seja, $w=\lambda v$.

Corolário 1.1. Se ve w são dois vetores tipo tempo que estão no mesmo cone tipo tempo, existe um único $\varphi \geq 0$ tal que

$$
\langle v, w\rangle_{1}=-\|v\|_{1}\|w\|_{1} \cosh \varphi .
$$

O número $\varphi$ é chamado o ângulo hiperbólico entre ve e w.

Demonstração. O fato de $v$ e $w$ estarem no mesmo cone tipo tempo implica que $\langle v, w\rangle_{1}<0$. Logo, segue da desigualdade reversa de Cauchy que

$$
-\langle v, w\rangle_{1} \geq\|v\|_{1}\|w\|_{1} \Rightarrow-\frac{\langle v, w\rangle_{1}}{\|v\|_{1}\|w\|_{1}} \geq 1
$$

Como a função cosh : $[0, \infty) \rightarrow[1, \infty)$ é injetiva, então, para todo $y \geq 1$, existe um único $\varphi \geq 0$ tal que $y=\cosh \varphi$. Portanto, pela equação (1.22), existe um único $\varphi \in[0, \infty)$ tal que

$$
-\frac{\langle v, w\rangle_{1}}{\|v\|_{1}\|w\|_{1}}=\cosh \varphi
$$

de onde segue o resultado.

De maneira análoga, podemos definir o ângulo hiperbólico entre dois vetores $v$ e $w$ que não estão no mesmo cone tipo tempo.

Corolário 1.2. Se ve e w não estão no mesmo cone tipo tempo, existe um único $\varphi \geq 0$, chamado o ângulo hiperbólico entre ve w, tal que

$$
\langle v, w\rangle_{1}=\|v\|_{1}\|w\|_{1} \cosh \varphi
$$


Para finalizar o estudo das propriedades básicas do espaço de Lorentz-Minkowski, vamos falar um pouco sobre o produto vetorial neste ambiente.

Definição 1.23. Sejam u e v vetores em $\mathbb{L}^{3}$. O produto vetorial Lorentziano de u e v (nesta ordem), denotado por $u \wedge_{1} v$, é o unico vetor que satisfaz

$$
\left\langle u \wedge_{1} v, w\right\rangle_{1}=\operatorname{det}(u, v, w), \forall w \in \mathbb{L}^{3},
$$

onde

$$
\operatorname{det}(u, v, w)=\left|\begin{array}{ccc}
u_{1} & u_{2} & u_{3} \\
v_{1} & v_{2} & v_{3} \\
w_{1} & w_{2} & w_{3}
\end{array}\right|
$$

e $u_{i}, v_{i}, w_{i}, i=1,2,3$, são as coordenadas dos vetores $u, v, w$, respectivamente, na base canônica.

A bilinearidade de $g=\langle,\rangle_{1}$ garante a existência e unicidade de $u \wedge_{1} v$. Fazendo $w$ variar entre os vetores da base canônica $\left\{e_{1}, e_{2}, e_{3}\right\}$, obtemos as coordenadas de $u \wedge_{1} v$ :

$$
u \wedge_{1} v=\left(u_{2} v_{3}-u_{3} v_{2}, u_{3} v_{1}-u_{1} v_{3}, u_{2} v_{1}-u_{1} v_{2}\right) .
$$

Ou ainda, numa outra notação:

$$
u \wedge_{1} v=\left|\begin{array}{ccc}
i & j & -k \\
u_{1} & u_{2} & u_{3} \\
v_{1} & v_{2} & v_{3}
\end{array}\right| .
$$

Pode-se observar que, dados vetores $u$ e $v$ em $\mathbb{L}^{3}$ não degenerados linearmente independentes, $\left\{u, v, u \wedge_{1} v\right\}$ é uma base de $\mathbb{L}^{3}$. Porém, tal base não necessariamente está orientada positivamente. O caráter de causalidade de $u$ e $v$ é que determinará a orientação. Se um dos vetores é tipo tempo, como $u \wedge_{1} v$ é ortogonal a $u$ e a $v$ (como veremos a seguir), então $u \wedge_{1} v$ é tipo espaço e, assim, $\operatorname{det}\left(u, v, u \wedge_{1} v\right)=\left\langle u \wedge_{1} v, u \wedge_{1} v\right\rangle_{1}>0$ e $\left\{u, v, u \wedge_{1} v\right\}$ é positiva. Se ambos $u$ e $v$ são tipo espaço, então $u \wedge_{1} v$ é tipo tempo. $\operatorname{Logo}, \operatorname{det}\left(u, v, u \wedge_{1} v\right)<0$ e $\left\{u, v, u \wedge_{1} v\right\}$ é negativa.

Proposição 1.12. Sejam $u, v, x, y \in \mathbb{L}^{3}$. São válidas as seguintes propriedades.

1. $u \wedge_{1} v=-v \wedge_{1} u$.

2. $u \wedge_{1} v \perp$ ue $u \wedge_{1} v \perp v$.

3. $u \wedge_{1} v=0$ se, e somente se, $u$ e $v$ são proporcionais.

4. $\left\langle u \wedge_{1} v, x \wedge_{1} y\right\rangle_{1}=\left|\begin{array}{ll}\langle u, y\rangle_{1} & \langle v, y\rangle_{1} \\ \langle u, x\rangle_{1} & \langle v, x\rangle_{1}\end{array}\right|$. 
5. $\left\langle u \wedge_{1} v, x\right\rangle_{1}=\left\langle x \wedge_{1} u, v\right\rangle_{1}$.

6. $x \wedge_{1}\left(u \wedge_{1} v\right)=\langle x, u\rangle_{1} v-\langle x, v\rangle_{1} u$.

As demonstrações seguem das propriedades do determinante.

\subsubsection{Curvas Paramétricas}

Definição 1.24. Uma curva paramétrica (ou parametrizada) em $\mathbb{L}^{3}$ é uma aplicação $\alpha: I \rightarrow \mathbb{L}^{3}$ definida num intervalo aberto $I \subset \mathbb{R}$.

Dizemos que $\alpha(t)=(x(t), y(t), z(t))$ é diferenciável se todas as derivadas das funções coordenadas $x(t), y(t), z(t)$ existem e são contínuas (isto é, cada função é de classe $C^{\infty}$ ). Consideraremos daqui em diante curvas diferenciáveis, mesmo que isto não seja mencionado.

Definição 1.25. Seja $\alpha: I \rightarrow \mathbb{L}^{3}$ uma curva parametrizada diferenciável. Classificamos $\alpha$ num ponto $t_{0} \in$ I como:

1. Tipo tempo, se $\alpha^{\prime}\left(t_{0}\right)$ é um vetor tipo tempo.

2. Tipo luz (ou nula), se $\alpha^{\prime}\left(t_{0}\right)$ é um vetor tipo luz.

3. Tipo espaço, se $\alpha^{\prime}\left(t_{0}\right)$ é um vetor tipo espaço.

Observamos que uma curva $\alpha$ não precisa ter o mesmo caráter de causalidade em todo o seu domínio. Por exemplo, se $\alpha(t)=\left(\cosh t, t^{2}, \sinh t\right), t \in \mathbb{R}$, então $\alpha^{\prime}(t)=(\sinh t, 2 t, \cosh t)$ $\mathrm{e}$

$$
\left\langle\alpha^{\prime}(t), \alpha^{\prime}(t)\right\rangle_{1}=\sinh ^{2} t+4 t^{2}-\cosh ^{2} t=4 t^{2}-1 \text {. }
$$

Assim, $\alpha$ é tipo tempo em $\left(-\frac{1}{2}, \frac{1}{2}\right)$, tipo luz em $\left\{-\frac{1}{2}, \frac{1}{2}\right\}$ e tipo espaço em $\left(-\infty,-\frac{1}{2}\right) \cup\left(\frac{1}{2}, \infty\right)$. Observamos também que, se $\alpha$ é tipo tempo em $t_{0}$ e tipo espaço em $t_{1}$ então, necessariamente, existe $t_{2} \in I$ onde $\alpha$ é tipo luz. Isto é garantido pelo Teorema do Valor Intermediário, já que $\alpha^{\prime}$ e $g=\langle,\rangle_{1}$ são funções contínuas. E, novamente pela continuidade destas funções, se $\alpha$ é tipo tempo (ou espaço) em $t_{0}$, então existe uma vizinhança $\left(t_{0}-\delta, t_{0}+\delta\right)$ onde $\alpha$ é tipo tempo (ou espaço).

Definição 1.26. Uma curva $\alpha$ é regular em $t_{0} \in I$ se $\alpha^{\prime}\left(t_{0}\right) \neq 0$. Dizemos simplesmente que $\alpha$ é regular se ela é regular em todo $t_{0} \in I$.

\section{Exemplo 1.2.}

1. A reta $\alpha(t)=p+t v, p, v \in \mathbb{L}^{3}$, tem o mesmo caráter de causalidade que $v$. 
2. A parábola $\alpha(t)=\left(t, t^{2}, t^{2}\right)$ é uma curva tipo espaço num plano tipo luz. De fato, $\alpha^{\prime}(t)=$ $(1,2 t, 2 t)$, que implica $\left\langle\alpha^{\prime}(t), \alpha^{\prime}(t)\right\rangle_{1}=1>0$. Além disso,

$$
\alpha(t)=t(1,0,0)+t^{2}(0,1,1) \in \operatorname{Span}\{(1,0,0),(0,1,1)\} .
$$

Denote $e_{1}=(1,0,0), e_{2}=(0,1,1)$ e $P=\operatorname{Span}\left\{e_{1}, e_{2}\right\}$. Note que Pé tipo luz, pois, dado $v \in P$, existem $\lambda, \mu \in \mathbb{R}$ tais que $v=\lambda e_{1}+\mu e_{2}$, que implica

$$
\left\langle e_{2}, v\right\rangle_{1}=\left\langle e_{2}, \lambda e_{1}+\mu e_{2}\right\rangle_{1}=0,
$$

isto é, $\left.g\right|_{P}$ é degenerada.

Observação 1.1. Toda curva tipo tempo ou tipo luz é regular. De fato, se a é tipo tempo (luz), então $\alpha^{\prime}(t)$ é um vetor tipo tempo (luz), para todo $t \in I$ e, portanto, não nulo, já que o vetor nulo é classificado como tipo espaço. Logo, $\alpha^{\prime}(t) \neq 0, \forall t \in I$.

Definição 1.27. Seja $\alpha: I \rightarrow \mathbb{L}^{3}$ uma curva parametrizada (regular). Definimos a função comprimento de arco de $\alpha$ a partir de um ponto $t_{0} \in I$ por

$$
S(t)=\int_{t_{0}}^{t}\left\|\alpha^{\prime}(r)\right\|_{1} d r .
$$

Dizemos que $\alpha$ está parametrizada pelo comprimento de arco se $\left\|\alpha^{\prime}(t)\right\|_{1}=1$, para todo $t \in I$.

Para curvas tipo tempo ou tipo espaço, mostra-se de maneira análoga ao que é feito no espaço Euclidiano que sempre existe uma reparametrização de $\alpha$ pelo comprimento de arco.

Para curvas tipo luz, temos que $\left\langle\alpha^{\prime}(t), \alpha^{\prime}(t)\right\rangle_{1}=0$, ou seja, não podemos parametrizar uma curva tipo luz pelo comprimento de arco. Mas, derivando $\left\langle\alpha^{\prime}(t), \alpha^{\prime}(t)\right\rangle_{1}=0$, obtemos $\left\langle\alpha^{\prime \prime}(t), \alpha^{\prime}(t)\right\rangle_{1}=0$. Pela Proposição 1.10, $\operatorname{Span}\left\{\alpha^{\prime}\right\}^{\perp}$ é um plano tipo luz que não contém vetores tipo tempo. Assim, $\alpha^{\prime \prime}$ é tipo luz ou tipo espaço. Se $\alpha^{\prime \prime}$ é tipo luz, então, pela Proposição 1.8, existe $\lambda \in \mathbb{R}$ tal que $\alpha^{\prime \prime}(t)=\lambda \alpha^{\prime}(t)$, o que implica $\alpha(t)=a e^{\lambda t}+b, \operatorname{com} a, b \in \mathbb{L}^{3},\langle a, a\rangle_{1}=0$. Isto é, $\alpha$ é parametrização de uma reta (tipo luz). Se $\alpha^{\prime \prime}$ é tipo espaço, existe uma reparametrização de $\alpha$ pelo pseudo-comprimento de arco, como mostra o lema a seguir.

Lema 1.5. Seja $\alpha: I \rightarrow \mathbb{L}^{3}$ uma curva tipo luz tal que $\alpha^{\prime \prime}(t)$ é um vetor tipo espaço para todo $t$ (isto é, $\alpha$ não é uma reta). Existe uma reparametrização de $\alpha$ dada por $\beta(s)=\alpha(\phi(s))$, para alguma função diferenciável $\phi$, tal que $\left\|\beta^{\prime \prime}(s)\right\|_{1}=1$. Dizemos que $\alpha$ está pseudo-parametrizada pelo comprimento de arco.

Demonstração. Seja $\phi: J \subset \mathbb{R} \rightarrow I$ uma função diferenciável. Dada $\beta(s)=\alpha(\phi(s))$, temos que

$$
\left\langle\beta^{\prime \prime}(s), \beta^{\prime \prime}(s)\right\rangle_{1}=\phi^{\prime}(s)^{4}\left\langle\alpha^{\prime \prime}(\phi(s)), \alpha^{\prime \prime}(\phi(s))\right\rangle_{1}=\phi^{\prime}(s)^{4}\left\|\alpha^{\prime \prime}(\phi(s))\right\|_{1}^{2} .
$$


Como buscamos $\beta$ tal que $\left\|\beta^{\prime \prime}(s)\right\|_{1}=1$, basta tomarmos $\phi$ como solução da equação diferencial

$$
\phi^{\prime}(s)=\frac{1}{\sqrt{\left\|\alpha^{\prime \prime}(\phi(s))\right\|_{1}}},
$$

garantida pela teoria de EDO.

\section{Curvatura e Torção}

Nosso objetivo agora é descrever uma base de $\mathbb{L}^{3}$ para cada ponto de uma curva regular $\alpha(s)$, cuja variação descreve a geometria da curva. Tal base é o triedro de Frenet que, a depender do caráter de causalidade da curva no ponto, pode mudar um pouco a sua configuração. Consideraremos a curva $\alpha$ parametrizada pelo comprimento de arco ou pelo pseudo-comprimento de arco, a depender do caso.

Definimos o vetor tangente a $\alpha$ em $s$ por

$$
t(s)=\alpha^{\prime}(s) .
$$

Observe que $t$ e $t^{\prime}$ são ortogonais, já que $\langle t, t\rangle_{1}$ é constante, igual a 0,1 ou -1 . Nos restringiremos a curvas em que $t^{\prime}(s) \neq 0$ e $t$ e $t^{\prime}$ não são proporcionais. Analisaremos cada caso separadamente a depender do caráter de causalidade da curva $\alpha$.

\section{Curvas Tipo Tempo}

Seja $\alpha$ uma curva tipo tempo. Então $\alpha$ é regular e podemos parametrizá-la pelo comprimento de arco. Considere o vetor tangente $t(s) \neq 0$ tal que $t^{\prime}(s) \neq 0$. Temos que $t^{\prime}(s)$ e $t(s)$ são vetores ortogonais e, assim, $t^{\prime}(s)$ é tipo espaço. Além disso, eles são linearmente independentes.

Definição 1.28. Considere a curva $\alpha$ tipo tempo.

1. Definimos a curvatura de a em s pelo número real positivo $\kappa(s)=\left\|t^{\prime}(s)\right\|_{1}$.

2. O vetor normal unitário em sé $n(s)=\frac{t^{\prime}(s)}{\kappa(s)}$.

3. O vetor binormal de $\alpha$ em séb(s) $=t(s) \wedge_{1} n(s)$.

4. A torção em sé o escalar $\tau(s)=\left\langle n^{\prime}(s), b(s)\right\rangle_{1}=-\left\langle b^{\prime}(s), n(s)\right\rangle_{1}$.

Observe que $b$ é tipo espaço e unitário, já que $b \in \operatorname{Span}\{t\}^{\perp} \mathrm{e}$

$$
\|b\|_{1}=\sqrt{\langle b, b\rangle_{1}}=\sqrt{\left\langle t \wedge_{1} n, t \wedge_{1} n\right\rangle_{1}}=1,
$$


onde a última igualdade segue do item 4 da Proposição 1.12. Note ainda que $b$ e $t$, bem como $b$ e $n$, são linearmente independentes. Obtemos assim, para cada $s \in I$, uma base ortonormal para $\mathbb{L}^{3},\{t, n, b\}$, denominada o triedro de Frenet em $s$. Note que $\{t, n, b\}$ está orientada positivamente, já que $\operatorname{det}(t, n, b)=\left\langle t \wedge_{1} n, b\right\rangle_{1}=\langle b, b\rangle_{1}=1>0$.

Analisaremos como ficam as Equações de Frenet que, assim como no caso Euclidiano, são expressões para os vetores $t^{\prime}, n^{\prime}, b^{\prime}$ na base $\{t, n, b\}$.

Temos, por definição, $t^{\prime}=\kappa n$. Para encontrarmos uma expressão para $n^{\prime}$ e $b^{\prime}$, escreva

$$
n^{\prime}=n_{1} t+n_{2} n+n_{3} b, \quad b^{\prime}=b_{1} t+b_{2} n+b_{3} b .
$$

Tomando o produto interno de $n^{\prime}$ com cada vetor da base, obtemos $-n_{1}=\left\langle n^{\prime}, t\right\rangle_{1}, n_{2}=$ $\left\langle n^{\prime}, n\right\rangle_{1}=0, n_{3}=\left\langle n^{\prime}, b\right\rangle_{1}=\tau$. Note que

$$
\langle t, n\rangle_{1}=0 \Rightarrow\left\langle t^{\prime}, n\right\rangle_{1}+\left\langle t, n^{\prime}\right\rangle_{1}=0 \Rightarrow\left\langle t, n^{\prime}\right\rangle_{1}=-\kappa .
$$

Segue que $n^{\prime}=\kappa t+\tau b$. Analogamente, obtemos $b_{1}=-\left\langle b^{\prime}, t\right\rangle_{1}=0, b_{2}=\left\langle b^{\prime}, n\right\rangle_{1}=-\tau$, $b_{3}=\left\langle b^{\prime}, b\right\rangle_{1}=0$ e, portanto, $b^{\prime}=-\tau n$. As equações de Frenet neste caso ficam

$$
\left\{\begin{array}{l}
t^{\prime}=\kappa n \\
n^{\prime}=\kappa t+\tau b \\
b^{\prime}=-\tau n .
\end{array}\right.
$$

\section{Curvas Tipo Espaço}

Seja $\alpha$ uma curva tipo espaço parametrizada pelo comprimento de arco. Sendo $t^{\prime} \perp t$ e $t$ tipo espaço, $t^{\prime}$ pode ser tipo tempo, tipo espaço ou tipo luz. Neste caso, o triedro e as equações de Frenet vão depender do caráter de $t^{\prime}$.

1. O vetor $t^{\prime}$ é tipo espaço: Vamos considerar $t^{\prime}(s) \neq 0$. As definições neste caso são iguais às anteriores, com exceção da torção $\tau$.

Definição 1.29. Seja a uma curva tipo espaço tal que t'é um vetor tipo espaço não nulo.

(a) Definimos a curvatura de a em s pelo número real positivo $\kappa(s)=\left\|t^{\prime}(s)\right\|_{1}$.

(b) O vetor normal unitário em sé $n(s)=\frac{t^{\prime}(s)}{\mathcal{K}(s)}$.

(c) O vetor binormal de $\alpha$ em sé $b(s)=t(s) \wedge_{1} n(s)$.

(d) A torção em sé o escalar $\tau(s)=-\left\langle n^{\prime}(s), b(s)\right\rangle_{1}=\left\langle b^{\prime}(s), n(s)\right\rangle_{1}$.

Temos que $t$ e $n$ são vetores tipo espaço, implicando que $b$ é tipo tempo. Assim, novamente temos uma base ortonormal $\{t, n, b\}$, desta vez orientada negativamente, pois $\operatorname{det}(t, n, b)=\left\langle t \wedge_{1} n, t \wedge_{1} n\right\rangle_{1}=\langle b, b\rangle_{1}=-1$. 
Escrevendo $n^{\prime}$ e $b^{\prime}$ nesta base e lembrando que $\langle t, t\rangle_{1}=\langle n, n\rangle_{1}=1$ e $\langle b, b\rangle_{1}=-1$, seguem as equações de Frenet:

$$
\left\{\begin{array}{l}
t^{\prime}=\kappa n \\
n^{\prime}=-\kappa t+\tau b \\
b^{\prime}=\tau n .
\end{array}\right.
$$

2. O vetor $t^{\prime}$ é tipo tempo: Neste caso, a base $\{t, n, b\}$ resultará orientada positivamente e será formada por dois vetores tipo espaço, e o normal tipo tempo, como podemos ver a partir da definição a seguir.

Definição 1.30. Seja a uma curva tipo espaço tal que t' é um vetor tipo tempo.

(a) Definimos a curvatura de a em s por $\kappa(s)=\left\|t^{\prime}(s)\right\|_{1}$.

(b) O vetor normal unitário é $n(s)=\frac{t^{\prime}(s)}{\kappa(s)}$.

(c) O vetor binormal de $\alpha$ é $b(s)=t(s) \wedge_{1} n(s)$.

(d) A torção é o escalar $\tau(s)=\left\langle n^{\prime}(s), b(s)\right\rangle_{1}=-\left\langle b^{\prime}(s), n(s)\right\rangle_{1}$.

Assim, obtemos

$$
\left\{\begin{array}{l}
t^{\prime}=\kappa n \\
n^{\prime}=\kappa t+\tau b \\
b^{\prime}=\tau n .
\end{array}\right.
$$

3. O vetor $t^{\prime}$ é tipo luz: Seja $\alpha$ uma curva tipo espaço parametrizada pelo comprimento de arco tal que $t^{\prime}$ é um vetor tipo luz. Assim, $\|t\|_{1}=1$ e $\left\|t^{\prime}\right\|_{1}=0$. Neste caso, não definimos curvatura. Os vetores normal e binormal e a torção são definidos a seguir.

Definição 1.31. Seja a curva tipo espaço com $t^{\prime}$ tipo luz.

(a) Definimos o vetor normal por $n=t^{\prime}$.

(b) O binormal b é o único vetor tipo luz tal que $\langle n, b\rangle_{1}=1 e b \perp t$.

(c) A torção é definida por $\tau=\left\langle n^{\prime}, b\right\rangle_{1}=-\left\langle b^{\prime}, n\right\rangle_{1}$.

Note que o vetor $b$ está bem definido pois, sendo $t$ tipo espaço, temos que $\operatorname{Span}\{t\}^{\perp}$ é tipo tempo. Logo, pela Proposição 1.9, existem dois vetores tipo luz linearmente independentes em $\operatorname{Span}\{t\}^{\perp}$. O vetor $n$ é tipo luz. Assim, podemos definir $b$ tipo luz de modo que $n$ e $b$ sejam linearmente independentes. Logo, $\langle n, b\rangle_{1} \neq 0$ e podemos escolher $\langle n, b\rangle_{1}=1$. 
Obtemos assim o triedro de Frenet $\{t, n, b\}$ formado por um vetor tipo espaço e dois vetores tipo luz e, neste caso, não é uma base ortonormal. Note que os vetores satisfazem

$$
t \perp n, t \perp b, n \not \perp b,\|t\|_{1}=1 \text {. }
$$

Definidas estas entidades geométricas, vejamos como ficam as equações de Frenet.

Por definição, $t^{\prime}=n$. Escrevendo $n^{\prime}=n_{1} t+n_{2} n+n_{3} b$ e fazendo o produto interno de $n^{\prime}$ com cada vetor da base, obtemos $n_{1}=n_{3}=0$ e $n_{2}=\tau$. Logo, $n^{\prime}=\tau n$. Analogamente, tem-se $b^{\prime}=-t-\tau b$. As equações de Frenet são, portanto,

$$
\left\{\begin{array}{l}
t^{\prime}=n \\
n^{\prime}=\tau n \\
b^{\prime}=-t-\tau b .
\end{array}\right.
$$

\section{Curvas Tipo Luz}

Neste tópico, consideraremos $\alpha$ uma curva pseudo-parametrizada pelo comprimento de arco, isto é, $t^{\prime}(s)$ é um vetor tipo espaço e unitário. O vetor tangente é $t=\alpha^{\prime}$, com $\|t\|=0$, e o normal é $n=t^{\prime}$. Em resumo:

Definição 1.32. Seja a uma curva tipo luz.

1. Definimos o vetor normal por $n=t^{\prime}$.

2. O binormal b é o único vetor tipo luz ortogonal a $n$ tal que $\langle t, b\rangle_{1}=1$.

3. A torção é definida por $\tau=\left\langle n^{\prime}, b\right\rangle_{1}=-\left\langle b^{\prime}, n\right\rangle_{1}$.

As equações de Frenet para este caso são deduzidas de maneira inteiramente análoga ao que já foi feito nos outros casos, ou seja, escreve-se os vetores $t^{\prime}, n^{\prime}$ e $b^{\prime}$ na base $t, n, b$ e tomase o produto interno destes vetores com cada vetor da base. Em seguida, basta aplicar as propriedades derivadas da definição de cada um, resultando em

$$
\left\{\begin{array}{l}
t^{\prime}=n \\
n^{\prime}=\tau t-b \\
b^{\prime}=-\tau n .
\end{array}\right.
$$

\subsubsection{Superfícies Paramétricas}

Definição 1.33. Uma superfície paramétrica regular no Espaço de Lorentz-Minkowski é uma aplicação $X: U \subset \mathbb{R}^{2} \rightarrow \mathbb{L}^{3}$, definida no aberto $U$ do $\mathbb{R}^{2}$, tal que 
1. X é diferenciável $\left(C^{\infty}\right)$.

2. Para todo $q=(u, v) \in U, d X_{q}: \mathbb{R}^{2} \rightarrow \mathbb{R}^{3}$ é injetiva.

Pelo Teorema da Função Inversa, $X$ é um difeomorfismo local. Sendo $d X_{q}: \mathbb{R}^{2} \rightarrow d X_{q}\left(\mathbb{R}^{2}\right)$ um isomorfismo, tem-se que $d X_{q}\left(\mathbb{R}^{2}\right)$ é um subespaço de $\mathbb{R}^{3}$ de dimensão 2 , chamado o plano tangente de $X$ em $p=X(q)$ e denotado por $T_{p} X$. Uma base para $T_{p} X$ é $\left\{d X_{q}\left(e_{1}\right), d X_{q}\left(e_{2}\right)\right\}=$ $\left\{X_{u}(q), X_{v}(q)\right\}, q=(u, v) \in U$.

Dado $p \in X$, o plano tangente $T_{p} X$ pode ser um subespaço tipo tempo, tipo espaço ou tipo luz, ou não ter caráter de causalidade definido. No caso em que $T_{p} X$ tem o mesmo caráter para todo $p \in X$, classificamos $X$ da seguinte maneira.

1. X é tipo espaço se $T_{p} X$ é tipo espaço para todo $p \in X(U)$.

2. X é tipo tempo se $T_{p} X$ é tipo tempo para todo $p \in X(U)$.

3. X é tipo luz se $T_{p} X$ é tipo luz para todo $p \in X(U)$.

Da definição de produto vetorial em $\mathbb{L}^{3}$, temos que $u \wedge_{1} v$ é ortogonal a $u$ e a $v$. Definimos assim o vetor normal unitário de $X$ em $p$, quando $X_{u} \wedge_{1} X_{v}$ é tipo tempo ou tipo espaço, por

$$
N(p)=\frac{X_{u} \wedge_{1} X_{v}}{\left\|X_{u} \wedge_{1} X_{v}\right\|_{1}}
$$

Um conceito muito importante para o estudo de curvas sobre superfícies é o triedro de Darboux. Seja $X$ uma superfície paramétrica tipo espaço ou tipo tempo e $\alpha$ uma curva sobre $X$ parametrizada pelo comprimento de arco. Para cada $p=\alpha(s)$, considere os vetores $t(s), N(s)$, $V(s)$, onde $t(s)$ é o vetor tangente a $\alpha, N(s)$ é o vetor normal à superfície e $V(s)=N(s) \wedge_{1} t(s)$. O triedro de Darboux é formado pelos vetores $t(s), N(s), V(s)$, os quais formam uma base ortonormal $\{t, V, N\}$.

\section{Superfícies Tipo Espaço}

Seja $X$ uma superfície tipo espaço. Note que, sendo $T_{p} X$ um plano tipo espaço, então $t$ é tipo espaço e $N$ é tipo tempo. Em particular, $\alpha$ é uma curva tipo espaço e, além disso, $V \perp N$ implica que $V$ é tipo espaço. A base $\{t, V, N\}$ é positiva, uma vez que

$$
\operatorname{det}(t, V, N)=\left\langle t \wedge_{1}\left(N \wedge_{1} t\right), N\right\rangle_{1}=\left\langle N \wedge_{1} t,\left(N \wedge_{1} t\right)\right\rangle_{1}=\langle V, V\rangle_{1}>0 .
$$

Vimos que neste caso, onde $\alpha$ é tipo espaço, o vetor $t^{\prime}$ pode ser tipo espaço, tipo luz ou tipo tempo. Decompondo o vetor $t^{\prime}=\vec{K}$ na base $\{t, V, N\}$, obtemos

$$
\vec{K}=-\langle\vec{K}, N\rangle_{1} N+\langle\vec{K}, V\rangle_{1} V=-\vec{K}_{n}+\vec{K}_{g}
$$

onde $\vec{K}_{n}=\langle\vec{K}, N\rangle_{1} N$ e $\vec{K}_{g}=\langle\vec{K}, V\rangle_{1} V$ são chamados, respectivamente, de vetor curvatura normal e vetor curvatura geodésica. 
Definição 1.34. Seja a uma curva regular (tipo espaço) sobre a superfície tipo espaço X que passa pelo ponto $p$. Definimos a curvatura normal $\kappa_{n}$, a curvatura geodésica $\kappa_{g}$ e a torção geodésica $\tau_{g}$ de $\alpha$ em $p$, respectivamente, por

$$
\kappa_{n}=-\langle\vec{K}, N\rangle_{1}, \quad \kappa_{g}=\langle\vec{K}, V\rangle_{1}, \quad \tau_{g}=\left\langle N^{\prime}, V\right\rangle_{1} .
$$

Note que

$$
\begin{aligned}
& \vec{K}_{n}=\langle\vec{K}, N\rangle_{1} N=-\kappa_{n} N \\
& \vec{K}_{g}=\langle\vec{K}, V\rangle_{1} V=\kappa_{g} V .
\end{aligned}
$$

As definições introduzidas garantem que $t^{\prime}=\kappa_{n} N+\kappa_{g} V$. Queremos ainda encontrar expressões para $V^{\prime}$ e $N^{\prime}$ na base $\{t, V, N\}$. Isto se dá de maneira inteiramente análoga ao que fizemos para obter as equações de Frenet, resultando em

$$
\left\{\begin{aligned}
t^{\prime} & =\kappa_{n} N+\kappa_{g} V \\
V^{\prime} & =-\kappa_{g} t+\tau_{g} N \\
N^{\prime} & =\kappa_{n} t+\tau_{g} V .
\end{aligned}\right.
$$

\section{Superfícies Tipo Tempo}

Se $X$ é tipo tempo, então $T_{p} X$ é um plano tipo tempo, o que implica que o vetor normal $N$ é tipo espaço e a curva $\alpha$ sobre $X$ pode ser tipo espaço, tipo tempo ou tipo luz. Vamos analisar os casos tipo espaço e tipo tempo. O caráter de causalidade do vetor $V=N \wedge_{1} t$ e, portanto, a orientação da base $\{t, V, N\}$, dependerá do caráter de $\alpha$.

1. A curva $\alpha$ é tipo espaço: Neste caso, $t$ é tipo espaço, $N$ é tipo espaço, $V$ é tipo tempo e a base $\{t, V, N\}$ é negativa.

Definição 1.35. Seja a uma curva regular tipo espaço sobre a superfície tipo tempo X que passa pelo ponto $p$. Definimos a curvatura normal $\kappa_{n}$, a curvatura geodésica $\kappa_{g}$ e a torção geodésica $\tau_{g}$ de $\alpha$ em $p$, respectivamente, por

$$
\kappa_{n}=-\langle\vec{K}, N\rangle_{1}, \quad \kappa_{g}=-\langle\vec{K}, V\rangle_{1}, \quad \tau_{g}=-\left\langle N^{\prime}, V\right\rangle_{1} .
$$

Assim, temos as seguintes equações:

$$
\left\{\begin{aligned}
t^{\prime} & =\kappa_{g} V-\kappa_{n} N \\
V^{\prime} & =\kappa_{g} t+\tau_{g} N \\
N^{\prime} & =\kappa_{n} t+\tau_{g} V .
\end{aligned}\right.
$$


2. A curva $\alpha$ é tipo tempo: Supondo agora que $\alpha$ é tipo tempo, então $t$ é tipo tempo, $N$ é tipo espaço, $V$ é tipo espaço e a base $\{t, V, N\}$ é positiva.

Definição 1.36. Seja a uma curva regular tipo tempo sobre a superfície tipo tempo X que passa pelo ponto $p$. Definimos a curvatura normal $\kappa_{n}$, a curvatura geodésica $\kappa_{g}$ e a torção geodésica $\tau_{g}$ de $\alpha$ em $p$, respectivamente, por

$$
\kappa_{n}=\langle\vec{K}, N\rangle_{1}, \quad \kappa_{g}=\langle\vec{K}, V\rangle_{1}, \quad \tau_{g}=\left\langle N^{\prime}, V\right\rangle_{1}
$$

Escrevendo os vetores $t^{\prime}, V^{\prime}, N^{\prime}$ na base $\{t, V, N\}$, obtemos

$$
\left\{\begin{aligned}
t^{\prime} & =\kappa_{n} N+\kappa_{g} V \\
V^{\prime} & =\kappa_{g} t-\tau_{g} N \\
N^{\prime} & =\kappa_{n} t+\tau_{g} V .
\end{aligned}\right.
$$

Uma curva $\alpha$ sobre uma superfície $X$ no espaço $\mathbb{L}^{3}$ pode ser classificada como assintótica, geodésica ou linha de curvatura, como veremos na definição a seguir.

Definição 1.37. Seja $\alpha(s)=X(u(s)$,v(s)) uma curva regular, conexa, tipo espaço ou tipo tempo. Dizemos que a é:

1. Curva assintótica se $\kappa_{n} \equiv 0$.

2. Curva geodésica se $\kappa_{g} \equiv 0$.

3. Linha de curvatura se $\tau_{g} \equiv 0$.

Com isto, finalizamos este capítulo, onde tratamos de conceitos fundamentais para o estudo das curvas de interseção entre superfícies nos espaços Euclidiano e de Lorentz-Minkowski. 


\section{Capítulo 2}

\section{Curvas de Interseção entre duas Superfícies}

\section{no Espaço Euclidiano}

Baseados em [1], [4], [14] e [18], estudaremos neste capítulo as curvas de interseção entre duas superfícies paramétricas ou implícitas no Espaço Euclidiano. Em cada caso, serão analisadas as propriedades da curva de interseção nas duas possíveis situações: quando a interseção é do tipo transversal ou tangencial.

Seja $P$ um ponto de interseção entre duas superfícies. Dizemos que a interseção é do tipo transversal em $P$ quando os vetores normais de ambas as superfícies neste ponto não são paralelos. Caso contrário, dizemos que a interseção é do tipo tangencial.

As superfícies consideradas serão sempre regulares. Assim, se $X(u, v)$ é uma superfície paramétrica e $f(x, y, z)$ é uma superfície implícita, então $X_{u} \wedge X_{v} \neq 0$ e $\nabla f \neq 0$. Denotaremos os vetores normais das superfícies $X$ (paramétrica) e $f$ (implícita), respectivamente, por:

$$
N^{X}=\frac{X_{u} \wedge X_{v}}{\left\|X_{u} \wedge X_{v}\right\|} \quad \text { e } \quad N^{f}=\frac{\nabla f}{\|\nabla f\|} .
$$

\subsection{Interseção Transversal}

A seguir, trataremos dos três tipos de interseção transversal, a saber, Paramétrica-Paramétrica, Paramétrica-Implícita e Implícita-Implícita, analisando em cada caso expressões para o vetor tangente, curvatura, vetor curvatura, torção e derivadas de ordem superior.

\subsubsection{Interseção entre duas Superfícies Paramétricas}

Estudaremos neste tópico os conceitos relacionados às curvas de interseção transversal entre duas superfícies paramétricas, introduzidos em 1999 por Ye e Maekawa [18]. Considere 
$X\left(u_{1}, v_{1}\right)$ e $Y\left(u_{2}, v_{2}\right)$ superfícies paramétricas que se intersectam transversalmente ao longo de uma curva $\alpha$, a qual está parametrizada pelo comprimento de arco. Como vimos anteriormente, $N^{X} \nVdash N^{Y}$ ao longo de $\alpha$. Portanto, $N^{X} \wedge N^{Y} \neq 0$. Isto nos permite definir o vetor tangente como segue.

\section{Vetor Tangente}

Como $\alpha$ está sobre $X$ e sobre $Y$, então o vetor tangente $t=\alpha^{\prime}$ está sobre os planos tangentes a $X$ e a $Y$. Logo, $t \perp N^{X}$ e $t \perp N^{Y}$. Portanto, $t \| N^{X} \wedge N^{Y}$ e podemos definir

$$
t=\frac{N^{X} \wedge N^{Y}}{\left\|N^{X} \wedge N^{Y}\right\|}
$$

\section{Curvatura e Vetor Curvatura}

Inicialmente, vamos encontrar uma expressão para o vetor curvatura $\vec{K}$ em termos de alguns coeficientes conhecidos relacionados com as superfícies $X$ e $Y$.

Teorema 2.1. Sejam X e Y superfícies paramétricas que se intersectam transversalmente ao longo de uma curva $\alpha$. O vetor curvatura de $\alpha$ é dado por

$$
\vec{K}=\frac{\kappa_{n}^{X}-\kappa_{n}^{Y} \cos \theta}{\sin ^{2} \theta} N^{X}+\frac{\kappa_{n}^{Y}-\kappa_{n}^{X} \cos \theta}{\sin ^{2} \theta} N^{Y},
$$

onde

$$
\kappa_{n}^{X}=e^{X}\left(u_{1}^{\prime}\right)^{2}+2 f^{X} u_{1}^{\prime} v_{1}^{\prime}+g^{X}\left(v_{1}^{\prime}\right)^{2}, \quad \kappa_{n}^{Y}=e^{Y}\left(u_{2}^{\prime}\right)^{2}+2 f^{Y} u_{2}^{\prime} v_{2}^{\prime}+g^{Y}\left(v_{2}^{\prime}\right)^{2},
$$

e os parâmetros $u_{i}^{\prime}$ e $v_{i}^{\prime}, i=1,2$, são obtidos em termos dos coeficientes da primeira forma fundamental relativos a $X$ e a $Y$.

Demonstração. O vetor $\vec{K}=\alpha^{\prime \prime}$, sendo perpendicular a $t=\alpha^{\prime}$, deve estar no plano gerado por $N^{X}$ e $N^{Y}$. Assim, existem $\mu, \eta \in \mathbb{R}$ tais que

$$
\vec{K}=\mu N^{X}+\eta N^{Y}
$$

Denotando por $\kappa_{n}^{X}$ e $\kappa_{n}^{Y}$ as curvaturas normais no ponto de interseção $P$ do ponto de vista das superfícies $X$ e $Y$, respectivamente, temos da definição de curvatura normal (ver equação (1.6)) e pela equação (2.2) que

$$
\begin{aligned}
& \kappa_{n}^{X}=\mu\left\langle N^{X}, N^{X}\right\rangle+\eta\left\langle N^{X}, N^{Y}\right\rangle=\mu+\eta \cos \theta \\
& \kappa_{n}^{Y}=\mu\left\langle N^{X}, N^{Y}\right\rangle+\eta\left\langle N^{Y}, N^{Y}\right\rangle=\mu \cos \theta+\eta,
\end{aligned}
$$


onde $\theta$ é o ângulo entre $N^{X}$ e $N^{Y}$. Assim,

$$
\begin{aligned}
& \kappa_{n}^{X}=\mu+\eta \cos \theta \\
& \kappa_{n}^{Y}=\mu \cos \theta+\eta .
\end{aligned}
$$

Resolvendo o sistema (2.3) para $\mu$ e $\eta$, obtemos

$$
\mu=\frac{\kappa_{n}^{X}-\kappa_{n}^{Y} \cos \theta}{\sin ^{2} \theta} \quad \text { e } \quad \eta=\frac{\kappa_{n}^{Y}-\kappa_{n}^{X} \cos \theta}{\sin ^{2} \theta} .
$$

Substituindo em (2.2),

$$
\vec{K}=\frac{\kappa_{n}^{X}-\kappa_{n}^{Y} \cos \theta}{\sin ^{2} \theta} N^{X}+\frac{\kappa_{n}^{Y}-\kappa_{n}^{X} \cos \theta}{\sin ^{2} \theta} N^{Y} .
$$

Note que a equação (2.4) independe do tipo das superfícies (paramétricas ou implícitas). Para obtermos o vetor $\vec{K}$, basta conhecermos os valores das curvaturas normais em cada superfície. Analisaremos agora a curvatura normal da superfície paramétrica $X$. Para $Y$, as contas são análogas. Uma outra expressão para o vetor curvatura $\vec{K}=\alpha^{\prime \prime}$ é dada pela segunda das equações (1.4). Assim, fazendo o produto interno pelo vetor normal $N^{X}$ da superfície $X$, tem-se:

$$
\begin{aligned}
\kappa_{n}^{X} & =\left\langle\vec{K}, N^{X}\right\rangle=\underbrace{\left\langle X_{u_{1} u_{1}}, N^{X}\right\rangle}_{e^{X}}\left(u_{1}^{\prime}\right)^{2}+2 \underbrace{\left\langle X_{u_{1} v_{1}}, N^{X}\right\rangle}_{f^{X}} u_{1}^{\prime} v_{1}^{\prime}+\underbrace{\left\langle X_{v_{1} v_{1}}, N^{X}\right\rangle}_{g^{X}}\left(v_{1}^{\prime}\right)^{2} \\
& +\underbrace{\left\langle X_{u_{1}}, N^{X}\right\rangle}_{0} u_{1}^{\prime \prime}+\underbrace{\left\langle X_{v_{1}}, N^{X}\right\rangle}_{0} v_{1}^{\prime \prime} \\
& =e^{X}\left(u_{1}^{\prime}\right)^{2}+2 f^{X} u_{1}^{\prime} v_{1}^{\prime}+g^{X}\left(v_{1}^{\prime}\right)^{2},
\end{aligned}
$$

onde $e^{X}, f^{X}, g^{X}$ são os coeficientes da segunda forma fundamental de $X$.

Precisamos avaliar $u_{1}^{\prime}$ e $v_{1}^{\prime}$. É conhecido o vetor tangente $t=\frac{N^{X} \wedge N^{Y}}{\left\|N^{X} \wedge N^{Y}\right\|}$, pela expressão (2.1). Tomando-se o produto interno do vetor $\alpha^{\prime}$, dado nas equações (1.4), $\operatorname{com} X_{u_{1}}$ e $X_{v_{1}}$, tem-se

$$
\begin{aligned}
\left\langle t, X_{u_{1}}\right\rangle & =\left\langle X_{u_{1}}, X_{u_{1}}\right\rangle u_{1}^{\prime}+\left\langle X_{u_{1}}, X_{v_{1}}\right\rangle v_{1}^{\prime} \\
\left\langle t, X_{v_{1}}\right\rangle & =\left\langle X_{u_{1}}, X_{v_{1}}\right\rangle u_{1}^{\prime}+\left\langle X_{v_{1}}, X_{v_{1}}\right\rangle v_{1}^{\prime} .
\end{aligned}
$$

Logo,

$$
\begin{aligned}
& \left\langle t, X_{u_{1}}\right\rangle=E^{X} u_{1}^{\prime}+F^{X} v_{1}^{\prime} \\
& \left\langle t, X_{v_{1}}\right\rangle=F^{X} u_{1}^{\prime}+G^{X} v_{1}^{\prime},
\end{aligned}
$$

onde $E^{X}, F^{X}, G^{X}$ são os coeficientes da primeira forma fundamental de $X$. Resolvendo o sistema, obtemos

$$
u_{1}^{\prime}=\frac{\left\langle t, X_{u_{1}}\right\rangle G^{X}-\left\langle t, X_{v_{1}}\right\rangle F^{X}}{E^{X} G^{X}-\left(F^{X}\right)^{2}}, \quad v_{1}^{\prime}=\frac{\left\langle t, X_{v_{1}}\right\rangle E^{X}-\left\langle t, X_{u_{1}}\right\rangle F^{X}}{E^{X} G^{X}-\left(F^{X}\right)^{2}} .
$$


Analogamente, podemos obter $u_{2}^{\prime}$ e $v_{2}^{\prime}$. Substituindo $u_{i}^{\prime}$ e $v_{i}^{\prime}, i=1,2$, em (2.5), ficam determinadas as curvaturas normais das superfícies paramétricas $X$ e $Y$ e o teorema segue.

A curvatura é obtida fazendo-se $\kappa=\sqrt{\langle\vec{K}, \vec{K}\rangle}$.

\section{Torção e Derivada de Ordem Superior}

Lembremos que, derivando a expressão $\alpha^{\prime \prime}=\kappa n$, obtemos pelas equações de Frenet

$$
\alpha^{\prime \prime \prime}=-\kappa^{2} t+\kappa^{\prime} n+\kappa \tau b,
$$

o que implica que a torção $\tau$ pode ser escrita como

$$
\tau=\frac{\left\langle\alpha^{\prime \prime \prime}, b\right\rangle}{\kappa}
$$

Temos assim o seguinte teorema.

Teorema 2.2. Sejam X e Y superfícies paramétricas que se intersectam transversalmente ao longo de uma curva $\alpha$. A torção de a é dada por

$$
\tau=\frac{\left\langle\alpha^{\prime \prime \prime}, b\right\rangle}{\kappa}
$$

onde

$$
\alpha^{\prime \prime \prime}=-\kappa^{2} t+\frac{\lambda_{n}^{X}-\lambda_{n}^{Y} \cos \theta}{\sin ^{2} \theta} N^{X}+\frac{\lambda_{n}^{Y}-\lambda_{n}^{X} \cos \theta}{\sin ^{2} \theta} N^{Y}
$$

e $\lambda_{n}^{X}, \lambda_{n}^{Y}$ são obtidos em termos dos coeficientes da primeira e da segunda forma fundamental de X e de $Y$.

Demonstração. Note que $N^{X}$ e $N^{Y}$ geram o plano normal (o plano gerado pelos vetores $n$ e $b$ ). Assim, o termo $\kappa^{\prime} n+\kappa \tau b$ na expressão (2.7), por estar no plano normal, pode ser escrito como

$$
\kappa^{\prime} n+\kappa \tau b=\gamma N^{X}+\delta N^{Y},
$$

para alguns $\gamma, \delta \in \mathbb{R}$. Logo,

$$
\alpha^{\prime \prime \prime}=-\kappa^{2} t+\gamma N^{X}+\delta N^{Y} .
$$

Denotando por $\lambda_{n}$ a projeção do vetor $\alpha^{\prime \prime \prime}$ sobre o vetor normal $N$, tem-se pela equação anterior

$$
\lambda_{n}^{X}=\left\langle\alpha^{\prime \prime \prime}, N^{X}\right\rangle=-\kappa^{2} \underbrace{\left\langle t, N^{X}\right\rangle}_{0}+\gamma \underbrace{\left\langle N^{X}, N^{X}\right\rangle}_{1}+\delta \underbrace{\left\langle N^{Y}, N^{X}\right\rangle}_{\cos \theta}=\gamma+\delta \cos \theta .
$$

Analogamente, calculamos $\lambda_{n}^{Y}=\left\langle\alpha^{\prime \prime \prime}, N^{Y}\right\rangle$, obtendo por fim:

$$
\lambda_{n}^{X}=\gamma+\delta \cos \theta, \quad \lambda_{n}^{Y}=\gamma \cos \theta+\delta
$$


Resolvendo este sistema para $\gamma$ e $\delta$, temos

$$
\gamma=\frac{\lambda_{n}^{X}-\lambda_{n}^{Y} \cos \theta}{\sin ^{2} \theta}, \quad \delta=\frac{\lambda_{n}^{Y}-\lambda_{n}^{X} \cos \theta}{\sin ^{2} \theta}
$$

Substituindo em (2.8),

$$
\alpha^{\prime \prime \prime}=-\kappa^{2} t+\frac{\lambda_{n}^{X}-\lambda_{n}^{Y} \cos \theta}{\sin ^{2} \theta} N^{X}+\frac{\lambda_{n}^{Y}-\lambda_{n}^{X} \cos \theta}{\sin ^{2} \theta} N^{Y} .
$$

Assim, resta-nos avaliar $\lambda_{n}^{X}$ e $\lambda_{n}^{Y}$ para obtermos uma boa expressão para $\alpha^{\prime \prime \prime}$ e, portanto, para $\tau$. Mais uma vez, temos uma equação que independe do fato de as superfícies $X$ e $Y$ serem paramétricas ou implícitas. O que determinará a expressão para $\alpha^{\prime \prime \prime}$ em cada caso será o valor de $\lambda_{n}$. Faremos as contas aqui para o caso considerado: superfícies paramétricas. Novamente, a análise será feita para a superfície $X$, sendo que, para $Y$, esta segue de maneira análoga. Omitiremos os subscritos e sobrescritos que caracterizam a superfície $X$ por simplicidade. Do sistema (1.4),

$$
\begin{aligned}
\lambda_{n}= & \left\langle\alpha^{\prime \prime \prime}, N\right\rangle \\
= & \left\langle X_{u u u}, N\right\rangle\left(u^{\prime}\right)^{3}+3\left\langle X_{u u v}, N\right\rangle\left(u^{\prime}\right)^{2} v^{\prime}+3\left\langle X_{u v v}, N\right\rangle u^{\prime}\left(v^{\prime}\right)^{2}+ \\
& \left\langle X_{v v v}, N\right\rangle\left(v^{\prime}\right)^{3}+3\left[\left\langle X_{u u}, N\right\rangle u^{\prime} u^{\prime \prime}+\left\langle X_{u v}, N\right\rangle\left(u^{\prime \prime} v^{\prime}+u^{\prime} v^{\prime \prime}\right)+\right. \\
& \left.\left\langle X_{v v}, N\right\rangle v^{\prime} v^{\prime \prime}\right]+\left\langle X_{u}, N\right\rangle u^{\prime \prime \prime}+\left\langle X_{v}, N\right\rangle v^{\prime \prime \prime} \\
= & 3\left[e u^{\prime} u^{\prime \prime}+f\left(u^{\prime \prime} v^{\prime}+u^{\prime} v^{\prime \prime}\right)+g v^{\prime} v^{\prime \prime}\right]+\tilde{\Lambda}
\end{aligned}
$$

onde

$$
\tilde{\Lambda}=\left\langle X_{u u u}, N\right\rangle\left(u^{\prime}\right)^{3}+3\left\langle X_{u u v}, N\right\rangle\left(u^{\prime}\right)^{2} v^{\prime}+3\left\langle X_{u v v}, N\right\rangle u^{\prime}\left(v^{\prime}\right)^{2}+\left\langle X_{v v v}, N\right\rangle\left(v^{\prime}\right)^{3}
$$

Precisamos avaliar $u^{\prime \prime}$ e $v^{\prime \prime}$. Para isto, façamos o produto interno da expressão de $\alpha^{\prime \prime}$, dada nas equações (1.4), com $X_{u}$ e $X_{v}$ :

$$
\begin{aligned}
& \left\langle\alpha^{\prime \prime}, X_{u}\right\rangle=\left\langle X_{u u}, X_{u}\right\rangle\left(u^{\prime}\right)^{2}+2\left\langle X_{u v}, X_{u}\right\rangle u^{\prime} v^{\prime}+\left\langle X_{v v}, X_{u}\right\rangle\left(v^{\prime}\right)^{2}+\left\langle X_{u}, X_{u}\right\rangle u^{\prime \prime}+\left\langle X_{u}, X_{v}\right\rangle v^{\prime \prime} \\
& \left\langle\alpha^{\prime \prime}, X_{v}\right\rangle=\left\langle X_{u u}, X_{v}\right\rangle\left(u^{\prime}\right)^{2}+2\left\langle X_{u v}, X_{v}\right\rangle u^{\prime} v^{\prime}+\left\langle X_{v v}, X_{v}\right\rangle\left(v^{\prime}\right)^{2}+\left\langle X_{u}, X_{v}\right\rangle u^{\prime \prime}+\left\langle X_{v}, X_{v}\right\rangle v^{\prime \prime}
\end{aligned}
$$

Lembremos que $\alpha^{\prime \prime}=\vec{K}$ e

$$
\begin{gathered}
\text { - } E=\left\langle X_{u}, X_{u}\right\rangle \Rightarrow\left\{\begin{array}{l}
\left\langle X_{u u}, X_{u}\right\rangle=\frac{E_{u}}{2} \\
\left\langle X_{u v}, X_{u}\right\rangle=\frac{E_{v}}{2} .
\end{array}\right. \\
\text { - } F=\left\langle X_{u}, X_{v}\right\rangle \Rightarrow\left\{\begin{array}{l}
\left\langle X_{u u}, X_{v}\right\rangle=F_{u}-\frac{E_{v}}{2} \\
\left\langle X_{v v}, X_{u}\right\rangle=F_{v}-\frac{G_{u}}{2} .
\end{array}\right.
\end{gathered}
$$


- $G=\left\langle X_{v}, X_{v}\right\rangle \Rightarrow\left\{\begin{array}{l}\left\langle X_{u v}, X_{v}\right\rangle=\frac{G_{u}}{2} \\ \left\langle X_{v v}, X_{v}\right\rangle=\frac{G_{v}}{2} .\end{array}\right.$

Portanto,

$$
\begin{aligned}
& \left\langle\vec{K}, X_{u}\right\rangle=\frac{E_{u}}{2}\left(u^{\prime}\right)^{2}+E_{v} u^{\prime} v^{\prime}+\left(F_{v}-\frac{G_{u}}{2}\right)\left(v^{\prime}\right)^{2}+E u^{\prime \prime}+F v^{\prime \prime} \\
& \left\langle\vec{K}, X_{v}\right\rangle=\left(F_{u}-\frac{E_{v}}{2}\right)\left(u^{\prime}\right)^{2}+G_{u} u^{\prime} v^{\prime}+\frac{G_{v}}{2}\left(v^{\prime}\right)^{2}+F u^{\prime \prime}+G v^{\prime \prime} .
\end{aligned}
$$

Resolvendo o sistema para $u^{\prime \prime}$ e $v^{\prime \prime}$, obtém-se

$$
\begin{aligned}
u^{\prime \prime}= & \frac{1}{E G-F^{2}}\left[\left(\left(F_{u}-\frac{E_{v}}{2}\right) F-\frac{E_{u}}{2} G\right)\left(u^{\prime}\right)^{2}+\left(G_{u} F-E_{v} G\right) u^{\prime} v^{\prime}+\left(\frac{G_{v}}{2} F-\left(F_{v}-\frac{G_{u}}{2}\right) G\right)\left(v^{\prime}\right)^{2}\right. \\
& \left.+\left\langle\vec{K}, X_{u}\right\rangle G-\left\langle\vec{K}, X_{v}\right\rangle F\right] \\
v^{\prime \prime}= & \frac{1}{E G-F^{2}}\left[\left(\frac{E_{u}}{2} F-\left(F_{u}-\frac{E_{v}}{2}\right) E\right)\left(u^{\prime}\right)^{2}+\left(E_{v} F-G_{u} E\right) u^{\prime} v^{\prime}+\left(\left(F_{v}-\frac{G_{u}}{2}\right) F-\frac{G_{v}}{2} E\right)\left(v^{\prime}\right)^{2}\right. \\
& \left.+\left\langle\vec{K}, X_{v}\right\rangle E-\left\langle\vec{K}, X_{u}\right\rangle F\right] .
\end{aligned}
$$

Substituindo estes valores em (2.10), obtemos o escalar $\lambda_{n}$ e, por fim, considerando a equação (2.9), determina-se o vetor $\alpha^{\prime \prime \prime}$, como queríamos.

O algoritmo introduzido para calcular a derivada de terceira ordem da curva de interseção $\alpha$ pode ser generalizado para calcular a derivada $\alpha^{(m)}, m \geq 4$. Suponha que os valores $\alpha^{(j)}$, $1 \leq j \leq m-1$ sejam conhecidos. Diferencie a equação (2.7) $m-3$ vezes para obter o vetor $\alpha^{(m)}$. A cada diferenciação, substitua $t^{\prime}, n^{\prime}$ e $b^{\prime}$ pelas expressões dadas nas equações de Frenet (1.1), o que implica

$$
\alpha^{(m)}=c_{t} t+c_{n} n+c_{b} b
$$

onde $c_{t}, c_{n}, c_{b}$ são coeficientes que dependem somente de $\kappa, \tau$ e de suas derivadas. Substitua o termo $c_{n} n+c_{b} b$ por $\gamma N^{X}+\delta N^{Y}$, visto que estes pertencem ao plano normal, o que mostra que os coeficientes $c_{n}$ e $c_{b}$ não precisam ser determinados no passo anterior. Disto, segue que

$$
\alpha^{(m)}=c_{t} t+\gamma N^{X}+\delta N^{Y}
$$

Observe que $c_{t}=\left\langle\alpha^{\prime \prime \prime}, t\right\rangle$ e pode ser escrito em termos das derivadas de $\alpha$ de ordem menor que $m$. Por exemplo, se $m=5$, então $\left\langle\alpha^{(5)}, t\right\rangle=-4\left\langle\alpha^{(4)}, \alpha^{\prime \prime}\right\rangle-3\left\langle\alpha^{\prime \prime \prime}, \alpha^{\prime \prime \prime}\right\rangle$. De fato, derivando $\left\langle\alpha^{\prime \prime}, t\right\rangle=0$, obtemos $\left\langle\alpha^{\prime \prime \prime}, t\right\rangle=-\left\langle\alpha^{\prime \prime}, \alpha^{\prime \prime}\right\rangle$ e, derivando de ambos os lados da igualdade por mais duas vezes, chegamos ao resultado.

Continuando com o processo para obtermos o vetor $\alpha^{(m)}$, avalie $\lambda_{n}=\left\langle\alpha^{(m)}, N\right\rangle$, onde $\alpha^{(m)}$ deve ser obtido derivando-se o vetor $\alpha^{\prime \prime \prime}$ dado nas equações (1.4) e substituindo no resultado 
os valores de $u^{(m-1)}$ e $v^{(m-1)}$, que podem ser obtidos tomando-se o produto interno de $\alpha^{(m-1)}$ por $X_{u}$ e $X_{v}$ e resolvendo o sistema linear resultante, em que $X$ representa as duas superfícies paramétricas consideradas. Tome o produto interno da equação (2.12) com os vetores normais $N^{X}$ e $N^{Y}$, obtendo

$$
\lambda_{n}^{X}=\gamma+\delta \cos \theta, \quad \lambda_{n}^{Y}=\gamma \cos \theta+\delta
$$

Resolva o sistema linear para $\gamma$ e $\delta$ e substitua na equação (2.13), resultando em

$$
\alpha^{(m)}=c_{t} t+\frac{\lambda_{n}^{X}-\lambda_{n}^{Y} \cos \theta}{\sin ^{2} \theta} N^{X}+\frac{\lambda_{n}^{Y}-\lambda_{n}^{X} \cos \theta}{\sin ^{2} \theta} N^{Y} .
$$

\subsubsection{Interseção entre Superfícies Paramétricas e Implícitas}

Os conceitos apresentados a seguir fazem referência ao trabalho desenvolvido por Soliman et al. [14], onde foram estudadas as curvas de interseção transversal e tangencial entre uma superfície paramétrica e uma superfície implícita. O estudo sobre as interseções tangenciais para este caso será apresentado na Subseção 2.2.2.

Considere $X(u, v)$ superfície paramétrica, onde $(u, v)$ pertence ao aberto $U \subset \mathbb{R}^{2}$, e a superfície implícita $f(x, y, z)=0$, ambas regulares. Seja $\alpha$ a curva de interseção entre $X$ e $f$. Então, $\alpha$ pode ser vista das seguintes maneiras:

$$
\begin{aligned}
& \alpha(s)=(x(s), y(s), z(s)) ; f(x(s), y(s), z(s))=0 \\
& \alpha(s)=X(u(s), v(s)) ;(u(s), v(s)) \in U .
\end{aligned}
$$

Assim, denotando $X=\left(X^{1}, X^{2}, X^{3}\right)$, temos

$$
\begin{aligned}
& x(s)=X^{1}(u(s), v(s)) \\
& y(s)=X^{2}(u(s), v(s)) \\
& z(s)=X^{3}(u(s), v(s)) .
\end{aligned}
$$

Portanto, na interseção, a superfície $f$ pode ser expressa como

$$
h(u, v)=f\left(X^{1}, X^{2}, X^{3}\right)=0
$$

e, portanto, $\alpha$ é dada por

$$
\alpha(s)=X(u(s), v(s)) ; h(u(s), v(s))=0 .
$$




\section{Vetor Tangente}

Diferenciando (2.15), vale $h_{u} u^{\prime}+h_{v} v^{\prime}=0$. Daí,

$$
v^{\prime}=-\frac{h_{u}}{h_{v}} u^{\prime}, \text { se } h_{v} \neq 0
$$

Sendo $\alpha(s)=X(u(s), v(s))$ e $t=\alpha^{\prime}(s)$, tem-se:

$$
1=\|t\|^{2}=\left\langle X_{u} u^{\prime}+X_{v} v^{\prime}, X_{u} u^{\prime}+X_{v} v^{\prime}\right\rangle=E\left(u^{\prime}\right)^{2}+2 F u^{\prime} v^{\prime}+G\left(v^{\prime}\right)^{2} .
$$

Logo

$$
E\left(u^{\prime}\right)^{2}+2 F u^{\prime} v^{\prime}+G\left(v^{\prime}\right)^{2}=1 .
$$

Substituindo (2.16) em (2.17), segue que

$$
\begin{aligned}
& E\left(u^{\prime}\right)^{2}+2 F u^{\prime}\left(-\frac{h_{u}}{h_{v}} u^{\prime}\right)+G \frac{h_{u}^{2}}{h_{v}^{2}}\left(u^{\prime}\right)^{2}=1 \\
& \left(u^{\prime}\right)^{2} \frac{1}{h_{v}^{2}}\left(h_{v}^{2} E-2 h_{u} h_{v} F+h_{u}^{2} G\right)=1 \\
& \left(u^{\prime}\right)^{2}=h_{v}^{2}\left(h_{v}^{2} E-2 h_{u} h_{v} F+h_{u}^{2} G\right)^{-1} .
\end{aligned}
$$

Portanto,

$$
u^{\prime}=h_{v}\left(h_{v}^{2} E-2 h_{u} h_{v} F+h_{u}^{2} G\right)^{-\frac{1}{2}} .
$$

Substituindo em (2.16):

$$
v^{\prime}=-h_{u}\left(h_{v}^{2} E-2 h_{u} h_{v} F+h_{u}^{2} G\right)^{-\frac{1}{2}}
$$

Em resumo, temos que

$$
\begin{aligned}
& u^{\prime}=h_{v}\left(h_{v}^{2} E-2 h_{u} h_{v} F+h_{u}^{2} G\right)^{-\frac{1}{2}} \\
& v^{\prime}=-h_{u}\left(h_{v}^{2} E-2 h_{u} h_{v} F+h_{u}^{2} G\right)^{-\frac{1}{2}}
\end{aligned}
$$

Assim:

$$
\begin{aligned}
t & =X_{u} u^{\prime}+X_{v} v^{\prime} \\
& =X_{u} h_{v}\left(h_{v}^{2} E-2 h_{u} h_{v} F+h_{u}^{2} G\right)^{-\frac{1}{2}}-X_{v} h_{u}\left(h_{v}^{2} E-2 h_{u} h_{v} F+h_{u}^{2} G\right)^{-\frac{1}{2}} \\
& =\left(X_{u} h_{v}-X_{v} h_{u}\right)\left(h_{v}^{2} E-2 h_{u} h_{v} F+h_{u}^{2} G\right)^{-\frac{1}{2}} .
\end{aligned}
$$

Denote $\xi=X_{u} h_{v}-X_{v} h_{u}$ e note que $\|\xi\|=\left(h_{v}^{2} E-2 h_{u} h_{v} F+h_{u}^{2} G\right)^{\frac{1}{2}} \neq 0$. Logo,

$$
t=\frac{\xi}{\|\xi\|}
$$




\section{Curvatura e Vetor Curvatura}

A seguir, encontraremos uma expressão para o vetor curvatura $\vec{K}$ da curva de interseção $\alpha$.

Teorema 2.3. Considere a curva de interseção transversal $\alpha$ entre uma superfície paramétrica

$$
X(u, v)=\left(X^{1}(u, v), X^{2}(u, v), X^{3}(u, v)\right)
$$

e uma superfície implícita

$$
f(x, y, z)=0 .
$$

Sendo $h(u, v)=f\left(X^{1}, X^{2}, X^{3}\right)=0$ e $\xi=X_{u} h_{v}-X_{v} h_{u}$ o vetor curvatura da curva de interseção transversal entre a superfície paramétrica $X$ e a superfície implícita $f$ é dado por

$$
\vec{K}=\frac{\xi^{\prime}\|\xi\|^{2}-\xi\left\langle\xi^{\prime}, \xi\right\rangle}{\|\xi\|^{3}}
$$

onde $\xi^{\prime}=\|\xi\|^{-1}\left[h_{v}^{2} X_{u u}+h_{u}^{2} X_{v v}-2 h_{u} h_{v} X_{u v}+\left(h_{u v} h_{v}-h_{v v} h_{u}\right) X_{u}+\left(h_{u v} h_{u}-h_{u u} h_{v}\right) X_{v}\right]$.

Demonstração. Derivando o vetor tangente dado na equação (2.19), obtemos

$$
\vec{K}=\alpha^{\prime \prime}=\frac{\xi^{\prime}\|\xi\|-\xi\|\xi\|^{\prime}}{\|\xi\|^{2}}=\frac{\xi^{\prime}\|\xi\|^{2}-\xi\left\langle\xi^{\prime}, \xi\right\rangle}{\|\xi\|^{3}} .
$$

Sendo $\xi=X_{u} h_{v}-X_{v} h_{u}$, então

$$
\begin{aligned}
\xi^{\prime} & =h_{v}^{\prime} X_{u}+h_{v} X_{u}^{\prime}-\left(h_{u}^{\prime} X_{v}+h_{u} X_{v}^{\prime}\right) \\
& =\left(h_{u v} u^{\prime}+h_{v v} v^{\prime}\right) X_{u}+h_{v}\left(X_{u u} u^{\prime}+X_{u v} v^{\prime}\right)-\left[\left(h_{u u} u^{\prime}+h_{u v} v^{\prime}\right) X_{v}+h_{u}\left(X_{u v} u^{\prime}+X_{v v} v^{\prime}\right)\right] .
\end{aligned}
$$

De (2.18), segue que $u^{\prime}=\frac{h_{v}}{\|\xi\|}$ e $v^{\prime}=-\frac{h_{u}}{\|\xi\|}$. Logo, pela igualdade anterior:

$$
\begin{aligned}
\xi^{\prime}= & \frac{1}{\|\xi\|}\left[\left(h_{u v} h_{v}-h_{v v} h_{u}\right) X_{u}+h_{v}\left(X_{u u} h_{v}-X_{u v} h_{u}\right)-\left(h_{u u} h_{v}-h_{u v} h_{u}\right) X_{v}-\right. \\
& \left.h_{u}\left(X_{u v} h_{v}-X_{v v} h_{u}\right)\right] \\
= & \|\xi\|^{-1}\left[h_{v}^{2} X_{u u}+h_{u}^{2} X_{v v}-2 h_{u} h_{v} X_{u v}+\left(h_{u v} h_{v}-h_{v v} h_{u}\right) X_{u}+\right. \\
& \left.\left(h_{u v} h_{u}-h_{u u} h_{v}\right) X_{v}\right] .
\end{aligned}
$$

Isto prova o teorema.

Podemos assim obter expressões para os vetores normal e binormal e para a curvatura de $\alpha$, como mostra o resultado a seguir. 
Corolário 2.1. Sejam $n$ o vetor normal, $b$ o vetor binormal e $\kappa$ a curvatura da curva de interseção transversal $\alpha$ entre as superfícies paramétricas e implícitas $X$ e $f$. Então

$$
\begin{aligned}
n & =\frac{\|\xi\|^{2} \xi^{\prime}-\left\langle\xi^{\prime}, \xi\right\rangle \xi}{\|\| \xi\left\|^{2} \xi^{\prime}-\left\langle\xi^{\prime}, \xi\right\rangle \xi\right\|} \\
\kappa & =\frac{\|\| \xi\left\|^{2} \xi^{\prime}-\left\langle\xi^{\prime}, \xi\right\rangle \xi\right\|}{\|\xi\|^{3}} \\
b & =\frac{\xi}{\|\xi\|} \wedge \frac{\|\xi\|^{2} \xi^{\prime}-\left\langle\xi^{\prime}, \xi\right\rangle \xi}{\|\| \xi\left\|^{2} \xi^{\prime}-\left\langle\xi^{\prime}, \xi\right\rangle \xi\right\|} .
\end{aligned}
$$

Demonstração. Pela equação (2.19), $t=\frac{\xi}{\|\xi\|}$. Assim, estas expressões seguem diretamente das definições $n=\frac{\vec{K}}{\|\vec{K}\|}, \kappa=\|\vec{K}\|, b=t \wedge n$ e do Teorema 2.3.

\section{Torção e Derivada de Ordem Superior}

Nosso objetivo agora é obter uma expressão para a torção da curva de interseção transversal $\alpha$ entre a superfície paramétrica $X$ e a superfície implícita $f$. Isto é feito de acordo com o teorema a seguir.

Teorema 2.4. A torção da curva $\alpha$ de interseção transversal entre uma superfície paramétrica X e uma superfície implícita $f$ pode ser obtida da expressão $\tau=\frac{\left\langle\alpha^{\prime \prime \prime}, b\right\rangle}{\kappa}$, onde

$$
\begin{aligned}
\alpha^{\prime \prime \prime}(s) & =X_{u u u}\left(u^{\prime}\right)^{3}+3 X_{u u v}\left(u^{\prime}\right)^{2} v^{\prime}+3 X_{u v v} u^{\prime}\left(v^{\prime}\right)^{2}+X_{v v v}\left(v^{\prime}\right)^{3} \\
& +3\left[X_{u u} u^{\prime} u^{\prime \prime}+X_{u v}\left(u^{\prime \prime} v^{\prime}+u^{\prime} v^{\prime \prime}\right)+X_{v v} v^{\prime} v^{\prime \prime}\right]+X_{u} u^{\prime \prime \prime}+X_{v} v^{\prime \prime \prime},
\end{aligned}
$$

e $u^{\prime}, u^{\prime \prime}, u^{\prime \prime \prime}$ e $v^{\prime}, v^{\prime \prime}, v^{\prime \prime \prime}$ dependem das derivadas de $X=\left(X^{1}, X^{2}, X^{3}\right)$ e $h=f\left(X^{1}, X^{2}, X^{3}\right)$.

Demonstração. A expressão

$$
\begin{aligned}
\alpha^{\prime \prime \prime}(s) & =X_{u u u}\left(u^{\prime}\right)^{3}+3 X_{u u v}\left(u^{\prime}\right)^{2} v^{\prime}+3 X_{u v v} u^{\prime}\left(v^{\prime}\right)^{2}+X_{v v v}\left(v^{\prime}\right)^{3} \\
& +3\left[X_{u u} u^{\prime} u^{\prime \prime}+X_{u v}\left(u^{\prime \prime} v^{\prime}+u^{\prime} v^{\prime \prime}\right)+X_{v v} v^{\prime} v^{\prime \prime}\right]+X_{u} u^{\prime \prime \prime}+X_{v} v^{\prime \prime \prime},
\end{aligned}
$$

segue das equações (1.4). Vamos agora obter as derivadas de $u$ e $v$. Vimos que

$$
u^{\prime}=\frac{h_{v}}{\|\xi\|^{\prime}}, \quad v^{\prime}=-\frac{h_{u}}{\|\xi\|} .
$$

Derivando $u^{\prime}$, tem-se

$$
u^{\prime \prime}=\frac{h_{v}^{\prime}\|\xi\|-h_{v}\|\xi\|^{\prime}}{\|\xi\|^{2}}=\frac{h_{v}^{\prime}\|\xi\|^{2}-h_{v}\left\langle\xi^{\prime}, \xi\right\rangle}{\|\xi\|^{3}}=\frac{\left(h_{u v} u^{\prime}+h_{v v} v^{\prime}\right)\|\xi\|^{2}-h_{v}\left\langle\xi^{\prime}, \xi\right\rangle}{\|\xi\|^{3}} .
$$


Fazendo $h_{v}=u^{\prime}\|\xi\|:$

$$
u^{\prime \prime}=\left(\frac{h_{u v}}{\|\xi\|}-\frac{\left\langle\xi^{\prime}, \xi\right\rangle}{\|\xi\|^{2}}\right) u^{\prime}+\frac{h_{v v}}{\|\xi\|} v^{\prime}
$$

Analogamente, obtemos

$$
v^{\prime \prime}=-\frac{h_{u u}}{\|\xi\|} u^{\prime}-\left(\frac{h_{u v}}{\|\xi\|}+\frac{\left\langle\xi^{\prime}, \xi\right\rangle}{\|\xi\|^{2}}\right) v^{\prime}
$$

onde, por (2.20), $\xi^{\prime}$ pode ser representado da seguinte maneira:

$$
\begin{aligned}
\xi^{\prime} & =\frac{1}{\|\xi\|}\left[h_{u}\left(h_{u} X_{v v}-h_{v v} X_{u}+h_{u v} X_{v}-h_{v} X_{u v}\right)\right. \\
& \left.+h_{v}\left(h_{v} X_{u u}-h_{u} X_{u v}+h_{u v} X_{u}-h_{u u} X_{v}\right)\right] \\
& =\frac{h_{u}}{\|\xi\|}\left(h_{u} X_{v v}-h_{v v} X_{u}+h_{u v} X_{v}-h_{v} X_{u v}\right) \\
& +\frac{h_{v}}{\|\xi\|}\left(h_{v} X_{u u}-h_{u} X_{u v}+h_{u v} X_{u}-h_{u u} X_{v}\right) \\
& =-v^{\prime}\left(h_{u} X_{v v}-h_{v v} X_{u}+h_{u v} X_{v}-h_{v} X_{u v}\right) \\
& +u^{\prime}\left(h_{v} X_{u u}-h_{u} X_{u v}+h_{u v} X_{u}-h_{u u} X_{v}\right)
\end{aligned}
$$

ou seja,

$$
\xi^{\prime}=\left(h_{v} X_{u u}-h_{u} X_{u v}+h_{u v} X_{u}-h_{u u} X_{v}\right) u^{\prime}+\left(h_{v v} X_{u}-h_{u} X_{v v}-h_{u v} X_{v}+h_{v} X_{u v}\right) v^{\prime}
$$

Diferenciando (2.21),

$$
\begin{aligned}
u^{\prime \prime \prime} & =\left(\frac{h_{u v}}{\|\xi\|}-\frac{\left\langle\xi^{\prime}, \xi\right\rangle}{\|\xi\|^{2}}\right)^{\prime} u^{\prime}+\left(\frac{h_{u v}}{\|\xi\|}-\frac{\left\langle\xi^{\prime}, \xi\right\rangle}{\|\xi\|^{2}}\right) u^{\prime \prime}+\left(\frac{h_{v v}}{\|\xi\|}\right)^{\prime} v^{\prime}+\frac{h_{v v}}{\|\xi\|} v^{\prime \prime} \\
& =\left[\frac{\left(h_{u u v} u^{\prime}+h_{u v v} v^{\prime}\right)\|\xi\|^{2}-h_{u v}\left\langle\xi^{\prime}, \xi\right\rangle}{\|\xi\|^{3}}-\frac{\left(\left\langle\xi^{\prime \prime}, \xi\right\rangle+\left\|\xi^{\prime}\right\|^{2}\right)\|\xi\|^{3}-2\left\langle\xi^{\prime}, \xi\right\rangle^{2}\|\xi\|}{\|\xi\|^{5}}\right] u^{\prime} \\
& +\frac{\left(h_{u v v} u^{\prime}+h_{v v v} v^{\prime}\right)\|\xi\|^{2}-h_{v v}\left\langle\xi^{\prime}, \xi\right\rangle}{\|\xi\|^{3}} v^{\prime}+\left(\frac{h_{u v}}{\|\xi\|}-\frac{\left\langle\xi^{\prime}, \xi\right\rangle}{\|\xi\|^{2}}\right) u^{\prime \prime}+\frac{h_{v v}}{\|\xi\|} v^{\prime \prime}
\end{aligned}
$$

Logo,

$$
\begin{gathered}
u^{\prime \prime \prime}=\left(\frac{h_{u v}}{\|\xi\|}-\frac{\left\langle\xi^{\prime}, \xi\right\rangle}{\|\xi\|^{2}}\right) u^{\prime \prime}+\frac{h_{v v}}{\|\xi\|^{\prime \prime}} v^{\prime \prime} \frac{h_{u u v}}{\|\xi\|}\left(u^{\prime}\right)^{2}+\frac{h_{v v v}}{\|\xi\|}\left(v^{\prime}\right)^{2}+2 \frac{h_{u v v}}{\|\xi\|} u^{\prime} v^{\prime}+ \\
\left(-\frac{h_{u v}\left\langle\xi^{\prime}, \xi\right\rangle}{\|\xi\|^{3}}-\frac{\left\langle\xi^{\prime \prime}, \xi\right\rangle}{\|\xi\|^{2}}-\frac{\left\|\xi^{\prime}\right\|^{2}}{\|\xi\|^{2}}+2 \frac{\left\langle\xi^{\prime}, \xi\right\rangle^{2}}{\|\xi\|^{4}}\right) u^{\prime}-\frac{h_{v v}\left\langle\xi^{\prime}, \xi\right\rangle}{\|\xi\|^{3}} v^{\prime}
\end{gathered}
$$


E, analogamente, diferenciando (2.22)

$$
\begin{aligned}
v^{\prime \prime \prime} & =-\left(\frac{h_{u u}}{\|\xi\|}\right)^{\prime} u^{\prime}-\frac{h_{u u}}{\|\xi\|} u^{\prime \prime}-\left(\frac{h_{u v}}{\|\xi\|}+\frac{\left\langle\xi^{\prime}, \xi\right\rangle}{\|\xi\|^{2}}\right)^{\prime} v^{\prime}-\left(\frac{h_{u v}}{\|\xi\|}+\frac{\left\langle\xi^{\prime}, \xi\right\rangle}{\|\xi\|^{2}}\right) v^{\prime \prime} \\
& =-\frac{h_{u u}}{\|\xi\|} u^{\prime \prime}-\left(\frac{h_{u v}}{\|\xi\|}+\frac{\left\langle\xi^{\prime}, \xi\right\rangle}{\|\xi\|^{2}}\right) v^{\prime \prime}-\frac{h_{u u u}}{\|\xi\|}\left(u^{\prime}\right)^{2}-\frac{h_{u v v}}{\|\xi\|}\left(v^{\prime}\right)^{2}+\frac{h_{u u}\left\langle\xi^{\prime}, \xi\right\rangle}{\|\xi\|^{3}} u^{\prime}- \\
& 2 \frac{h_{u u v}}{\|\xi\|} u^{\prime} v^{\prime}+\left(\frac{h_{u v}\left\langle\xi^{\prime}, \xi\right\rangle}{\|\xi\|^{3}}-\frac{\left\langle\xi^{\prime \prime}, \xi\right\rangle}{\|\xi\|^{2}}-\frac{\left\|\xi^{\prime}\right\|^{2}}{\|\xi\|^{2}}+2 \frac{\left\langle\xi^{\prime}, \xi\right\rangle^{2}}{\|\xi\|^{4}}\right) v^{\prime},
\end{aligned}
$$

onde, por (2.23),

$$
\begin{aligned}
\xi^{\prime \prime}= & \left(h_{u v} X_{u}+h_{v} X_{u u}-h_{u u} X_{v}-h_{u} X_{u v}\right) u^{\prime \prime}+\left(h_{v v} X_{u}+h_{v} X_{u v}-h_{u v} X_{v}-h_{u} X_{v v}\right) v^{\prime \prime}+ \\
& \left(h_{u u v} X_{u}+2 h_{u v} X_{u u}+h_{v} X_{u u u}-h_{u u u} X_{v}-2 h_{u u} X_{u v}-h_{u} X_{u u v}\right)\left(u^{\prime}\right)^{2}+ \\
& \left(h_{v v v} X_{u}+2 h_{v v} X_{u v}+h_{v} X_{u v v}-h_{u v v} X_{v}-2 h_{u v} X_{v v}-h_{u} X_{v v v}\right)\left(v^{\prime}\right)^{2}+ \\
& 2\left(h_{u v v} X_{u}-h_{u u v} X_{v}+h_{v v} X_{u u}-h_{u u} X_{v v}+h_{v} X_{u u v}-h_{u} X_{u v v}\right) u^{\prime} v^{\prime}
\end{aligned}
$$

De maneira similar, podemos calcular as derivadas de ordem superior da curva de interseção. Ou seja, para encontrar a derivada $\alpha^{(m)}$, derivamos a expressão de $u^{\prime \prime \prime}$ e $v^{\prime \prime \prime} m-3$ vezes para obtermos $u^{(4)}, v^{(4)}, \ldots, u^{(m)}, v^{(m)}$. Em seguida, derivando a expressão $\alpha^{\prime \prime \prime} m-3$ vezes, obtemos $\alpha^{(m)}$ em função de $X$ e suas derivadas parciais até ordem $m$. Por fim, substituímos os valores conhecidos na expressão de $\alpha^{(m)}$ obtida no passo anterior.

\subsubsection{Interseção entre duas Superfícies Implícitas}

Até agora, consideramos a curva de interseção parametrizada pelo comprimento de arco. Nesta seção, porém, seguindo a notação de [1], que é a referência principal para o desenvolvimento desta subseção, o parâmetro de tal curva não necessariamente será o comprimento de arco $s$. Por isso, a notação utilizada para a derivada de uma curva $\alpha$ com respeito a $s$ será $\alpha^{\prime}(s)=\frac{d \alpha}{d s}, \alpha^{\prime \prime}(s)=\frac{d^{2} \alpha}{d s^{2}}, \alpha^{\prime \prime \prime}(s)=\frac{d^{3} \alpha}{d s^{3}}$ e $\alpha^{(n)}(s)=\frac{d^{n} \alpha}{d s^{n}}$, se $n \geq 4$. E, para uma curva $\beta$ com um parâmetro arbitrário $u$, denotamos $\dot{\beta}(u)=\frac{d \beta}{d u}, \ddot{\beta}(u)=\frac{d^{2} \beta}{d u^{2}}, \dddot{\beta}(u)=\frac{d^{3} \beta}{d u^{3}}$ e $\beta^{(n)}(u)=\frac{d^{n} \beta}{d u^{n}}$, para $n \geq 4$. Para avaliarmos as propriedades geométricas da curva de interseção entre duas superfícies implícitas, faremos uso do Teorema da Função Implícita [8]:

Teorema 2.5 (Teorema da Função Implícita). Sejam $f, g: A \subset \mathbb{R}^{3} \rightarrow \mathbb{R}$ funções de classe $C^{k}$, $k \geq 1$, definidas no aberto $A$ do $\mathbb{R}^{3}$ e $P_{0}=\left(x_{0}, y_{0}, z_{0}\right) \in A$ tal que $f\left(P_{0}\right)=c_{1}$ e g $\left(P_{0}\right)=c_{2}$. Se $\frac{\partial(f, g)}{\partial(y, z)}\left(P_{0}\right) \neq 0$, então existem abertos $U, V$ tais que $P_{0} \subset U \times V \subset A$ e, para todo $x \in U$, existe um 
único $(y, z)=(y(x), z(x)) \in V$ tal que

$$
\begin{aligned}
& f(x, y(x), z(x))=c_{1} \\
& g(x, y(x), z(x))=c_{2} .
\end{aligned}
$$

Ao trabalharmos com superfícies na forma implícita, lidamos com funções $f$ e $h$ diferenciáveis definidas num aberto $A$ do $\mathbb{R}^{3}$ que satisfazem $f(x, y, z)=0$ e $h(x, y, z)=0$. Sob as hipóteses do Teorema da Função Implícita, poderemos escrever $y$ e $z$ em função de $x$, de modo que $f(x, y(x), z(x))=0$ e $h(x, y(x), z(x))=0$, e a curva de interseção pode ser escrita como $\beta(x)=(x, y(x), z(x))$, possibilitando que as contas sejam simplificadas.

Sejam $f(x, y, z)=0$ e $h(x, y, z)=0$ superfícies implícitas regulares com vetores normais dados, respectivamente, por

$$
N^{f}=\frac{\nabla f}{\|\nabla f\|}, \quad N^{h}=\frac{\nabla h}{\|\nabla h\|} .
$$

Suponha que $f$ e $h$ se intersectam transversalmente ao longo de uma curva. Denotaremos por $\alpha(s)$ a curva de interseção parametrizada pelo comprimento de arco e $\beta(u)$ uma curva que tem o mesmo traço que $\alpha$ e está parametrizada por um parâmetro $u$ qualquer. Assim,

$$
\beta(u)=\alpha(s(u)), \quad f(\beta(u))=0 \quad \text { e } \quad h(\beta(u))=0 .
$$

Olhando para $\beta$ como uma curva sobre a superfície $f$, tem-se que

$$
\langle\nabla f(\beta(u)), \dot{\beta}(u)\rangle=0
$$

Ou, escrevendo na forma matricial,

$$
[\nabla f][\dot{\beta}]=[0]
$$

onde

$$
\begin{aligned}
{[\nabla f] } & =\left[\begin{array}{lll}
f_{x}(\beta(u)) & f_{y}(\beta(u)) & f_{z}(\beta(u))
\end{array}\right] \\
{[\dot{\beta}] } & =\left[\begin{array}{c}
\dot{x}(u) \\
\dot{y}(u) \\
\dot{z}(u)
\end{array}\right]
\end{aligned}
$$

Inicialmente, encontraremos algumas expressões envolvendo as derivadas de $\beta$ até ordem quatro, as quais serão úteis futuramente. Derivando (2.24),

$$
[\dot{\nabla} f][\dot{\beta}]+[\nabla f][\ddot{\beta}]=[0] .
$$


Observe que $[\dot{\nabla} f]=[\dot{\beta}]^{T} H$, onde

$$
H=\left[\begin{array}{ccc}
f_{x x} & f_{x y} & f_{x z} \\
f_{x y} & f_{y y} & f_{y z} \\
f_{x z} & f_{y z} & f_{z z}
\end{array}\right]
$$

é a matriz Hessiana da função $f$. De fato,

$$
\begin{aligned}
& {[\nabla f]=\left[f_{x}(\beta(u)) \quad f_{y}(\beta(u)) \quad f_{z}(\beta(u))\right] \Rightarrow} \\
& {[\dot{\nabla} f]=\left[f_{x x} \dot{x}+f_{x y} \dot{y}+f_{x z} \dot{z} f_{x y} \dot{x}+f_{y y} \dot{y}+f_{y z} \dot{z} \quad f_{x z} \dot{x}+f_{y z} \dot{y}+f_{z z} \dot{z}\right]=[\dot{\beta}]^{T} H .}
\end{aligned}
$$

Portanto, de (2.25)

$$
[\dot{\beta}]^{T} H[\dot{\beta}]+[\nabla f][\ddot{\beta}]=[0],
$$

o que implica

$$
[\nabla f][\ddot{\beta}]=-[\dot{\beta}]^{T} H[\dot{\beta}] .
$$

Derivando (2.26) com respeito a $u$

$$
\begin{gathered}
{[\dot{\nabla} f][\ddot{\beta}]+[\nabla f][\ddot{\beta}]=-\left[[\ddot{\beta}]^{T} H[\dot{\beta}]+[\dot{\beta}]^{T}(\dot{H}[\dot{\beta}]+H[\ddot{\beta}])\right]} \\
{[\dot{\beta}]^{T} H[\ddot{\beta}]+[\nabla f][\ddot{\beta}]=-\left[[\ddot{\beta}]^{T} H[\dot{\beta}]+[\dot{\beta}]^{T}(\dot{H}[\dot{\beta}]+H[\ddot{\beta}])\right],} \\
\text { onde, sendo } H=\left[\begin{array}{ccc}
f_{x x} & f_{x y} & f_{x z} \\
f_{x y} & f_{y y} & f_{y z} \\
f_{x z} & f_{y z} & f_{z z}
\end{array}\right] \text {, temos que: } \\
\dot{H}=\left[\begin{array}{lll}
f_{x x x} \dot{x}+f_{x y x} \dot{y}+f_{x z x} \dot{z} & f_{x x y} \dot{x}+f_{x y y} \dot{y}+f_{x y z} \dot{z} & f_{x x z} \dot{x}+f_{x y z} \dot{y}+f_{x z z} \dot{z} \\
f_{x y x} \dot{x}+f_{y y x} \dot{y}+f_{y z x} \dot{z} & f_{x y y} \dot{x}+f_{y y y} \dot{y}+f_{y z y} \dot{z} & f_{x y z} \dot{x}+f_{y y z} \dot{y}+f_{y z z} \dot{z} \\
f_{x z x} \dot{x}+f_{y z x} \dot{y}+f_{z z x} \dot{z} & f_{x z y} \dot{x}+f_{y z y} \dot{y}+f_{z z y} \dot{z} & f_{x z z} \dot{x}+f_{y z z} \dot{y}+f_{z z z} \dot{z}
\end{array}\right] \\
=\left[\begin{array}{lll}
\left(f_{x x} \dot{x}+f_{x y} \dot{y}+f_{x z} \dot{z}\right)_{x} & \left(f_{x x} \dot{x}+f_{x y} \dot{y}+f_{x z} \dot{z}\right)_{y} & \left(f_{x x} \dot{x}+f_{x y} \dot{y}+f_{x z} \dot{z}\right)_{z} \\
\left(f_{x y} \dot{x}+f_{y y} \dot{y}+f_{y z} \dot{z}\right)_{x} & \left(f_{x y} \dot{x}+f_{y y} \dot{y}+f_{y z} \dot{z}\right)_{y} & \left(f_{x y} \dot{x}+f_{y y} \dot{y}+f_{y z} \dot{z}\right)_{z} \\
\left(f_{x z} \dot{x}+f_{y z} \dot{y}+f_{z z} \dot{z}\right)_{x} & \left(f_{x z} \dot{x}+f_{y z} \dot{y}+f_{z z} \dot{z}\right)_{y} & \left(f_{x z} \dot{x}+f_{y z} \dot{y}+f_{z z} \dot{z}\right)_{z}
\end{array}\right] \\
=\left[\begin{array}{lll}
\nabla\left(f_{x x} \dot{x}+f_{x y} \dot{y}+f_{x z} \dot{z}\right) \\
\nabla\left(f_{x y} \dot{x}+f_{y y} \dot{y}+f_{y z} \dot{z}\right) \\
\nabla\left(f_{x z} \dot{x}+f_{y z} \dot{y}+f_{z z} \dot{z}\right)
\end{array}\right] .
\end{gathered}
$$


Denotaremos esta última matriz por

$$
\left[\begin{array}{c}
\nabla\left(f_{x x} \dot{x}+f_{x y} \dot{y}+f_{x z} \dot{z}\right) \\
\nabla\left(f_{x y} \dot{x}+f_{y y} \dot{y}+f_{y z} \dot{z}\right) \\
\nabla\left(f_{x z} \dot{x}+f_{y z} \dot{y}+f_{z z} \dot{z}\right)
\end{array}\right]=\nabla\left[\begin{array}{c}
f_{x x} \dot{x}+f_{x y} \dot{y}+f_{x z} \dot{z} \\
f_{x y} \dot{x}+f_{y y} \dot{y}+f_{y z} \dot{z} \\
f_{x z} \dot{x}+f_{y z} \dot{y}+f_{z z} \dot{z}
\end{array}\right]
$$

Então, seguindo as notações introduzidas, tem-se que

$$
\dot{H}=\nabla(H[\dot{\beta}]) \text {. }
$$

Por simplicidade, usaremos a notação $\dot{H}$. Continuando, da equação (2.27), tem-se

$$
[\dot{\beta}]^{T} H[\ddot{\beta}]+[\nabla f][\dddot{\beta}]=-[\ddot{\beta}]^{T} H[\dot{\beta}]-[\dot{\beta}]^{T} \dot{H}[\dot{\beta}]-[\dot{\beta}]^{T} H[\ddot{\beta}] .
$$

Note que $[\ddot{\beta}]^{T} H[\dot{\beta}]=\left([\ddot{\beta}]^{T} H[\dot{\beta}]\right)^{T}$, pois esta é uma matriz quadrada de ordem 1 . Assim, sendo $H$ uma matriz simétrica,

$$
[\ddot{\beta}]^{T} H[\dot{\beta}]=\left([\ddot{\beta}]^{T} H[\dot{\beta}]\right)^{T}=[\dot{\beta}]^{T} H^{T}\left([\ddot{\beta}]^{T}\right)^{T}=[\dot{\beta}]^{T} H[\ddot{\beta}]
$$

Daí, por (2.29),

$$
\begin{aligned}
{[\dot{\beta}]^{T} H[\ddot{\beta}]+[\nabla f][\dddot{\beta}] } & =-[\dot{\beta}]^{T} H[\ddot{\beta}]-[\dot{\beta}]^{T} \dot{H}[\dot{\beta}]-[\dot{\beta}]^{T} H[\ddot{\beta}] \Rightarrow \\
{[\nabla f][\dddot{\beta}] } & =-3[\dot{\beta}]^{T} H[\ddot{\beta}]-[\dot{\beta}]^{T} \dot{H}[\dot{\beta}] .
\end{aligned}
$$

Observe que, sendo $H$ uma matriz simétrica, então $\dot{H}$ também é simétrica e, portanto,

$$
\dot{H}^{T}=\dot{H}
$$

Analogamente ao que fizemos na equação (2.30), tem-se que $[\ddot{\beta}]^{T} \dot{H}[\dot{\beta}]=[\dot{\beta}]^{T} \dot{H}[\ddot{\beta}]$. Assim, derivando (2.31),

$$
\begin{aligned}
{[\dot{\nabla} f][\dddot{\beta}]+[\nabla f]\left[\beta^{(4)}\right] } & =-3\left[[\ddot{\beta}]^{T} H[\ddot{\beta}]+[\dot{\beta}]^{T}(\dot{H}[\ddot{\beta}]+H[\dddot{\beta}])\right]-[\ddot{\beta}]^{T} \dot{H}[\dot{\beta}]-[\dot{\beta}]^{T}(\ddot{H}[\dot{\beta}]+\dot{H}[\ddot{\beta}]) \\
& =-3[\ddot{\beta}]^{T} H[\ddot{\beta}]-3[\dot{\beta}]^{T} \dot{H}[\ddot{\beta}]-3[\dot{\beta}]^{T} H[\dddot{\beta}]-[\dot{\beta}]^{T} \dot{H}[\ddot{\beta}] \\
& -[\dot{\beta}]^{T} \ddot{H}[\dot{\beta}]-[\dot{\beta}]^{T} \dot{H}[\ddot{\beta}]
\end{aligned}
$$

Vamos agora calcular uma expressão para $\ddot{H}$. Lembre que, pela segunda igualdade de (2.28), temos

$$
\dot{H}=\left[\begin{array}{cccc}
\left(f_{x x} \dot{x}+f_{x y} \dot{y}+f_{x z} \dot{z}\right)_{x} & \left(f_{x x} \dot{x}+f_{x y} \dot{y}+f_{x z} \dot{z}\right)_{y} & \left(f_{x x} \dot{x}+f_{x y} \dot{y}+f_{x z} \dot{z}\right)_{z} \\
\left(f_{x y} \dot{x}+f_{y y} \dot{y}+f_{y z} \dot{z}\right)_{x} & \left(f_{x y} \dot{x}+f_{y y} \dot{y}+f_{y z} \dot{z}\right)_{y} & \left(f_{x y} \dot{x}+f_{y y} \dot{y}+f_{y z} \dot{z}\right)_{z} \\
\left(f_{x z} \dot{x}+f_{y z} \dot{y}+f_{z z} \dot{z}\right)_{x} & \left(f_{x z} \dot{x}+f_{y z} \dot{y}+f_{z z} \dot{z}\right)_{y} & \left(f_{x z} \dot{x}+f_{y z} \dot{y}+f_{z z} \dot{z}\right)_{z}
\end{array}\right]
$$


Denote as derivadas parciais de cada função entre parênteses por $\ell_{i j}, i, j=1,2,3$, de modo que

$$
\dot{H}=\left[\begin{array}{lll}
\ell_{11} & \ell_{12} & \ell_{13} \\
\ell_{21} & \ell_{22} & \ell_{23} \\
\ell_{31} & \ell_{32} & \ell_{33}
\end{array}\right]
$$

Para todo $i, j=1,2,3$, temos o seguinte

$$
\frac{d \ell_{i j}}{d u}=\frac{d \ell_{i j}}{d x} \frac{d x}{d u}+\frac{d \ell_{i j}}{d y} \frac{d y}{d u}+\frac{d \ell_{i j}}{d z} \frac{d z}{d u}=\left(\ell_{i j}\right)_{x} \dot{x}+\left(\ell_{i j}\right)_{y} \dot{y}+\left(\ell_{i j}\right)_{z} \dot{z}
$$

Portanto, a matriz $\ddot{H}$ é dada por

$$
\left[\begin{array}{lll}
\left(\ell_{11}\right)_{x} \dot{x}+\left(\ell_{11}\right)_{y} \dot{y}+\left(\ell_{11}\right)_{z} \dot{z} & \left(\ell_{12}\right)_{x} \dot{x}+\left(\ell_{12}\right)_{y} \dot{y}+\left(\ell_{12}\right)_{z} \dot{z} & \left(\ell_{13}\right)_{x} \dot{x}+\left(\ell_{13}\right)_{y} \dot{y}+\left(\ell_{13}\right)_{z} \dot{z} \\
\left(\ell_{21}\right)_{x} \dot{x}+\left(\ell_{21}\right)_{y} \dot{y}+\left(\ell_{21}\right)_{z} \dot{z} & \left(\ell_{22}\right)_{x} \dot{x}+\left(\ell_{22}\right)_{y} \dot{y}+\left(\ell_{22}\right)_{z} \dot{z} & \left(\ell_{23}\right)_{x} \dot{x}+\left(\ell_{23}\right)_{y} \dot{y}+\left(\ell_{23}\right)_{z} \dot{z} \\
\left(\ell_{31}\right)_{x} \dot{x}+\left(\ell_{31}\right)_{y} \dot{y}+\left(\ell_{31}\right)_{z} \dot{z} & \left(\ell_{32}\right)_{x} \dot{x}+\left(\ell_{32}\right)_{y} \dot{y}+\left(\ell_{32}\right)_{z} \dot{z} & \left(\ell_{33}\right)_{x} \dot{x}+\left(\ell_{33}\right)_{y} \dot{y}+\left(\ell_{33}\right)_{z} \dot{z}
\end{array}\right]
$$

o que implica

$$
\ddot{H}=\nabla\left[\begin{array}{lll}
\ell_{11} & \ell_{12} & \ell_{13} \\
\ell_{21} & \ell_{22} & \ell_{23} \\
\ell_{31} & \ell_{32} & \ell_{33}
\end{array}\right]\left[\begin{array}{l}
\dot{x} \\
\dot{y} \\
\dot{z}
\end{array}\right]=\nabla \dot{H}
$$

Por simplicidade, continuaremos denotando esta matriz por $\ddot{H}$. Assim, segue de (2.32) que

$$
[\nabla f]\left[\beta^{(4)}\right]=-4[\dot{\beta}]^{T} H[\dddot{\beta}]-3[\ddot{\beta}]^{T} H[\ddot{\beta}]-5[\dot{\beta}]^{T} \dot{H}[\ddot{\beta}]-[\dot{\beta}]^{T} \ddot{H}[\dot{\beta}] .
$$

\section{Vetor Tangente}

Assuma que $f$ e $h$ têm derivadas de primeira ordem contínuas e um dos determinantes $\frac{\partial(f, g)}{\partial(x, y)}, \frac{\partial(f, g)}{\partial(x, z)}, \frac{\partial(f, g)}{\partial(y, z)}$ seja não nulo (sem perda de generalidade, considere $\left.\frac{\partial(f, g)}{\partial(y, z)} \neq 0\right)$ em um ponto $P_{0}$ sobre a curva $\beta$. Então, pelo Teorema da Função Implícita, existem abertos $U, V$ tais que $P_{0} \in U \times V$ e, para todo $x \in U$, existe um único $(y, z)=(y(x), z(x)) \in V$ tal que

$$
f(x, y(x), z(x))=0 \quad \text { e } \quad h(x, y(x), z(x))=0 .
$$

Logo, sendo $\beta$ uma curva sobre $f$ e $h$, podemos escrever

$$
\beta(x)=(x, y(x), z(x)) ; f(\beta(x))=0, h(\beta(x))=0 .
$$


Por (2.24), $[\nabla f][\dot{\beta}]=[0]$ e $[\nabla h][\dot{\beta}]=[0]$. Pela equação anterior, derivando $\beta$ com respeito a $x$, tem-se

$$
\dot{\beta}=(1, \dot{y}, \dot{z})
$$

Portanto,

$$
\left[\begin{array}{lll}
f_{x} & f_{y} & f_{z} \\
h_{x} & h_{y} & h_{z}
\end{array}\right]\left[\begin{array}{l}
1 \\
\dot{y} \\
\dot{z}
\end{array}\right]=\left[\begin{array}{l}
0 \\
0
\end{array}\right]
$$

Vamos resolver este sistema para $\dot{y}$ e $\dot{z}$.

$$
\left[\begin{array}{lll}
f_{x} & f_{y} & f_{z} \\
h_{x} & h_{y} & h_{z}
\end{array}\right]\left[\begin{array}{c}
1 \\
\dot{y} \\
\dot{z}
\end{array}\right]=\left[\begin{array}{l}
0 \\
0
\end{array}\right] \Rightarrow\left[\begin{array}{ll}
f_{y} & f_{z} \\
h_{y} & h_{z}
\end{array}\right]\left[\begin{array}{l}
\dot{y} \\
\dot{z}
\end{array}\right]=\left[\begin{array}{c}
-f_{x} \\
-h_{x}
\end{array}\right] .
$$

Dessa forma,

$$
\begin{aligned}
{\left[\begin{array}{l}
\dot{y} \\
\dot{z}
\end{array}\right] } & =\left[\begin{array}{ll}
f_{y} & f_{z} \\
h_{y} & h_{z}
\end{array}\right]^{-1}\left[\begin{array}{l}
-f_{x} \\
-h_{x}
\end{array}\right] \\
& =\left(\operatorname{det}\left[\begin{array}{ll}
f_{y} & f_{z} \\
h_{y} & h_{z}
\end{array}\right]\right)^{-1}\left[\begin{array}{cc}
h_{z} & -f_{z} \\
-h_{y} & f_{y}
\end{array}\right]\left[\begin{array}{c}
-f_{x} \\
-h_{x}
\end{array}\right] \\
& =\left(\operatorname{det}\left[\begin{array}{ll}
f_{y} & f_{z} \\
h_{y} & h_{z}
\end{array}\right]\right)^{-1}\left[\begin{array}{c}
-h_{z} f_{x}+f_{z} h_{x} \\
h_{y} f_{x}-f_{y} h_{x}
\end{array}\right] .
\end{aligned}
$$

Assim,

$$
\left[\begin{array}{c}
\dot{y} \\
\dot{z}
\end{array}\right]=\frac{1}{A_{23}}\left[\begin{array}{r}
-A_{13} \\
A_{12}
\end{array}\right]
$$

onde, para $i, j=1,2,3$,

$$
A_{i j}=\operatorname{det}\left[\begin{array}{ll}
f_{i} & f_{j} \\
h_{i} & h_{j}
\end{array}\right], \quad \begin{aligned}
& f_{1}=f_{x}, f_{2}=f_{y}, f_{3}=f_{z} \\
& h_{1}=h_{x}, h_{2}=h_{y}, h_{3}=h_{z}
\end{aligned}
$$

Portanto, por (2.35),

$$
\dot{\beta}=\frac{\left(A_{23},-A_{13}, A_{12}\right)}{A_{23}} .
$$


Logo, a velocidade de $\beta$ é dada por

$$
\|\dot{\beta}\|=\frac{\sqrt{A_{23}^{2}+A_{12}^{2}+A_{13}^{2}}}{\left|A_{23}\right|} .
$$

Dessa forma, visto que o comprimento de arco e o vetor tangente de $\alpha$ são dados, respectivamente, por

$$
S(x)=\int_{x_{0}}^{x}\|\dot{\beta}\| d x, \quad t=\frac{\dot{\beta}}{\|\dot{\beta}\|},
$$

onde $x_{0}$ é o valor de $x$ no ponto $P_{0}$, segue que

$$
S(x)=\int_{x_{0}}^{x} \frac{\sqrt{A_{23}^{2}+A_{12}^{2}+A_{13}^{2}}}{\left|A_{23}\right|} d x
$$

$\mathrm{e}$

$$
t=\frac{\sigma\left(A_{23},-A_{13}, A_{12}\right)}{\sqrt{A_{23}^{2}+A_{12}^{2}+A_{13}^{2}}}, \quad \sigma=\left\{\begin{array}{rc}
1, & \text { se } A_{23}>0 \\
-1, & \text { se } A_{23}<0 .
\end{array}\right.
$$

\section{Curvatura e Vetor Curvatura}

Para obter uma expressão para o vetor curvatura, dado em (1.2), resta-nos conhecer $\ddot{\beta}$. Derivando (2.35) com respeito a $x$, tem-se

$$
\ddot{\beta}(x)=(0, \ddot{y}(x), \ddot{z}(x)) \text {. }
$$

Para descobrir os valores de $\ddot{y}$ e $\ddot{z}$, utilizaremos a equação (2.26) para $f$ e $h$. Denotando por $H_{1}$ e $H_{2}$ as matrizes Hessianas de $f$ e $h$, respectivamente, segue que

$$
\begin{aligned}
& {[\nabla f][\ddot{\beta}]=-[\dot{\beta}]^{T} H_{1}[\dot{\beta}]} \\
& {[\nabla h][\ddot{\beta}]=-[\dot{\beta}]^{T} H_{2}[\dot{\beta}] .}
\end{aligned}
$$

Assim,

$$
\left[\begin{array}{lll}
f_{x} & f_{y} & f_{z} \\
h_{x} & h_{y} & h_{z}
\end{array}\right]\left[\begin{array}{c}
0 \\
\ddot{y} \\
\ddot{z}
\end{array}\right]=\left[\begin{array}{c}
-[\dot{\beta}]^{T} H_{1}[\dot{\beta}] \\
-[\dot{\beta}]^{T} H_{2}[\dot{\beta}]
\end{array}\right] \Rightarrow\left[\begin{array}{ll}
f_{y} & f_{z} \\
h_{y} & h_{z}
\end{array}\right]\left[\begin{array}{c}
\ddot{y} \\
\ddot{z}
\end{array}\right]=\left[\begin{array}{c}
-[\dot{\beta}]^{T} H_{1}[\dot{\beta}] \\
-[\dot{\beta}]^{T} H_{2}[\dot{\beta}]
\end{array}\right],
$$

o que implica

$$
\left[\begin{array}{l}
\ddot{y} \\
\ddot{z}
\end{array}\right]=\frac{1}{A_{23}}\left[\begin{array}{rr}
h_{z} & -f_{z} \\
-h_{y} & f_{y}
\end{array}\right]\left[\begin{array}{l}
-[\dot{\beta}]^{T} H_{1}[\dot{\beta}] \\
-[\dot{\beta}]^{T} H_{2}[\dot{\beta}]
\end{array}\right] .
$$


Note que

$$
\begin{aligned}
& {[\dot{\beta}]^{T} H_{1}[\dot{\beta}]=\left[\begin{array}{ccc}
1 & \dot{y} & \dot{z}
\end{array}\right]\left[\begin{array}{ccc}
f_{x x} & f_{x y} & f_{x z} \\
f_{x y} & f_{y y} & f_{y z} \\
f_{x z} & f_{y z} & f_{z z}
\end{array}\right]\left[\begin{array}{c}
1 \\
\dot{y} \\
\dot{z}
\end{array}\right]} \\
& =\left[\begin{array}{lll}
f_{x x}+\dot{y} f_{x y}+\dot{z} f_{x z} & f_{x y}+\dot{y} f_{y y}+\dot{z} f_{y z} & f_{x z}+\dot{y} f_{y z}+\dot{z} f_{z z}
\end{array}\right]\left[\begin{array}{c}
1 \\
\dot{y} \\
\dot{z}
\end{array}\right] \\
& =\left[f_{x x}+\dot{y} f_{x y}+\dot{z} f_{x z}+\left(f_{x y}+\dot{y} f_{y y}+\dot{z} f_{y z}\right) \dot{y}+\left(f_{x z}+\dot{y} f_{y z}+\dot{z} f_{z z}\right) \dot{z}\right] \text {. }
\end{aligned}
$$

Portanto, definindo

$$
B_{i j k}=\operatorname{det}\left[\begin{array}{cc}
f_{i} & h_{i} \\
f_{j k} & h_{j k}
\end{array}\right]
$$

para $i, j, k=1,2,3$, tem-se

$$
\left[\begin{array}{l}
\ddot{y} \\
\ddot{z}
\end{array}\right]=-\frac{1}{A_{23}}\left[\begin{array}{r}
-B_{311}-(\dot{y})^{2} B_{322}-(\dot{z})^{2} B_{333}-2\left(\dot{y} B_{312}+\dot{z} B_{313}+\dot{y} \dot{z} B_{323}\right) \\
B_{211}+(\dot{y})^{2} B_{222}+(\dot{z})^{2} B_{233}+2\left(\dot{y} B_{212}+\dot{z} B_{213}+\dot{y} \dot{z} B_{223}\right)
\end{array}\right] .
$$

Lembremos de (2.37) que

$$
\left\{\begin{array}{l}
\dot{x}=1 \\
\dot{y}=-\frac{A_{13}}{A_{23}} \\
\dot{z}=\frac{A_{12}}{A_{23}} .
\end{array}\right.
$$

Substituindo na expressão anterior, segue que

$$
\begin{aligned}
\ddot{y} & =\frac{1}{A_{23}}\left[B_{311}+\frac{A_{13}^{2}}{A_{23}^{2}} B_{322}+\frac{A_{12}^{2}}{A_{23}^{2}} B_{333}+2\left(-\frac{A_{13}}{A_{23}} B_{312}+\frac{A_{12}}{A_{23}} B_{313}-\frac{A_{12} A_{13}}{A_{23}^{2}} B_{323}\right)\right] \\
& =\frac{1}{A_{23}^{3}}\left[A_{23}^{2} B_{311}+A_{13}^{2} B_{322}+A_{12}^{2} B_{333}+2\left(-A_{13} A_{23} B_{312}+A_{12} A_{23} B_{313}-A_{12} A_{13} B_{323}\right)\right] .
\end{aligned}
$$

Analogamente,

$$
\ddot{z}=-\frac{1}{A_{23}^{3}}\left[A_{23}^{2} B_{211}+A_{13}^{2} B_{222}+A_{12}^{2} B_{233}+2\left(-A_{13} A_{23} B_{212}+A_{12} A_{23} B_{213}-A_{12} A_{13} B_{223}\right)\right] .
$$


As equações (2.40) e (2.41) definem o vetor $\ddot{\beta}(x)=(0, \ddot{y}(x), \ddot{z}(x))$, como queríamos. Por fim, o vetor curvatura e a curvatura podem ser calculados usando as equações (1.2), (2.37) e (2.38):

$$
\begin{aligned}
& \vec{K}=\frac{\langle\dot{\beta}, \dot{\beta}\rangle \ddot{\beta}-\langle\dot{\beta}, \ddot{\beta}\rangle \dot{\beta}}{\|\dot{\beta}\|^{4}} \\
& =\frac{1}{\|\dot{\beta}\|^{4}}\left(-\dot{y} \ddot{y}-\dot{z} \ddot{z}, \frac{A_{23}^{2}+A_{13}^{2}+A_{12}^{2}}{A_{23}^{2}} \ddot{y}-(\dot{y} \ddot{y}+\dot{z} \ddot{z}) \dot{y}, \frac{\left.A_{23}^{2}+A_{13}^{2}+A_{12}^{2} \ddot{z}-(\dot{y} \ddot{y}+\dot{z} \ddot{z}) \dot{z}\right) .}{A_{23}^{2}} .\right.
\end{aligned}
$$

Notemos que

$$
\begin{aligned}
& \text { - } \frac{1}{\|\dot{\beta}\|^{4}}=\left(\frac{A_{23}^{2}}{A_{23}^{2}+A_{13}^{2}+A_{12}^{2}}\right)^{2} \\
& \text { - }-\dot{y} \ddot{y}-\dot{z} \ddot{z}=\frac{A_{13}}{A_{23}} \ddot{y}-\frac{A_{12}}{A_{23}} \ddot{z}=\frac{1}{A_{23}^{2}}\left(A_{13} A_{23} \ddot{y}-A_{12} A_{23} \ddot{z}\right) \text {, } \\
& \text { - } \frac{A_{23}^{2}+A_{13}^{2}+A_{12}^{2}}{A_{23}^{2}} \ddot{y}-(\dot{y} \ddot{y}+\dot{z} \ddot{z}) \dot{y}=\frac{A_{23}^{2}+A_{13}^{2}+A_{12}^{2}}{A_{23}^{2}} \ddot{y}-\frac{A_{13}^{2}}{A_{23}^{2}} \ddot{y}+\frac{A_{12} A_{13}}{A_{23}^{2}} \ddot{z} \\
& =\frac{1}{A_{23}^{2}}\left[\left(A_{23}^{2}+A_{12}^{2}\right) \ddot{y}+A_{12} A_{13} \ddot{z}\right] \text {, } \\
& \text { - } \frac{A_{23}^{2}+A_{13}^{2}+A_{12}^{2}}{A_{23}^{2}} \ddot{z}-(\dot{y} \ddot{y}+\dot{z} \ddot{z}) \dot{z}=\frac{A_{23}^{2}+A_{13}^{2}+A_{12}^{2}}{A_{23}^{2}} \ddot{z}+\frac{A_{12} A_{13}}{A_{23}^{2}} \ddot{y}-\frac{A_{12}^{2}}{A_{23}^{2}} \ddot{z} \\
& =\frac{1}{A_{23}^{2}}\left[\left(A_{23}^{2}+A_{13}^{2}\right) \ddot{z}+A_{12} A_{13} \ddot{y}\right] \text {. }
\end{aligned}
$$

Portanto, definindo $B=\frac{A_{23}}{A_{23}^{2}+A_{13}^{2}+A_{12}^{2}}$, então

$$
\vec{K}=B^{2}\left(A_{13} A_{23} \ddot{y}-A_{12} A_{23} \ddot{z},\left(A_{23}^{2}+A_{12}^{2}\right) \ddot{y}+A_{12} A_{13} \ddot{z}, A_{12} A_{13} \ddot{y}+\left(A_{23}^{2}+A_{13}^{2}\right) \ddot{z}\right) .
$$

Agora, vamos analisar a curvatura. Pelas equações (1.2),

$$
\kappa=\frac{\|\dot{\beta} \wedge \ddot{\beta}\|}{\|\dot{\beta}\|^{3}} .
$$

Das expressões $\dot{\beta}=\frac{\left(A_{23},-A_{13}, A_{12}\right)}{A_{23}}$ e $\ddot{\beta}=(0, \ddot{y}, \ddot{z})$, obtemos

$$
\begin{aligned}
\dot{\beta} \wedge \ddot{\beta} & =-\frac{1}{A_{23}}\left(A_{12} \ddot{y}+A_{13} \ddot{z}, A_{23} \ddot{z},-A_{23} \ddot{y}\right) \\
\|\dot{\beta} \wedge \ddot{\beta}\| & =\frac{1}{A_{23}} \sqrt{\left(A_{12}^{2}+A_{23}^{2}\right)(\ddot{y})^{2}+2 A_{12} A_{13} \ddot{y} \ddot{z}+\left(A_{13}^{2}+A_{23}^{2}\right)(\ddot{z})^{2}} \\
\|\dot{\beta}\|^{3} & =\frac{1}{A_{23}^{3}} \sqrt{\left(A_{23}^{2}+A_{13}^{2}+A_{12}^{2}\right)^{3}} .
\end{aligned}
$$


Logo,

$$
\kappa=A_{23}^{2} \sqrt{\frac{\left(A_{12}^{2}+A_{23}^{2}\right)(\ddot{y})^{2}+2 A_{12} A_{13} \ddot{y} \ddot{z}+\left(A_{13}^{2}+A_{23}^{2}\right)(\ddot{z})^{2}}{\left(A_{23}^{2}+A_{13}^{2}+A_{12}^{2}\right)^{3}}} .
$$

Em resumo, temos o seguinte resultado.

Teorema 2.6. Seja $\beta(x)$ a curva de interseção transversal entre as superfícies implícitas $f$ e $h$, dada pela equação (2.34). Então, o vetor curvatura e a curvatura de $\beta$ são dados, respectivamente, por

$$
\begin{aligned}
\vec{K} & =B^{2}\left(A_{13} A_{23} \ddot{y}-A_{12} A_{23} \ddot{z},\left(A_{23}^{2}+A_{12}^{2}\right) \ddot{y}+A_{12} A_{13} \ddot{z}, A_{12} A_{13} \ddot{y}+\left(A_{23}^{2}+A_{13}^{2}\right) \ddot{z}\right) \\
\kappa & =A_{23}^{2} \sqrt{\frac{\left(A_{12}^{2}+A_{23}^{2}\right)(\ddot{y})^{2}+2 A_{12} A_{13} \ddot{y} \ddot{z}+\left(A_{13}^{2}+A_{23}^{2}\right)(\ddot{z})^{2}}{\left(A_{23}^{2}+A_{13}^{2}+A_{12}^{2}\right)^{3}}},
\end{aligned}
$$

onde $B=\frac{A_{23}}{A_{23}^{2}+A_{13}^{2}+A_{12}^{2}}$ e $A_{i j}=\operatorname{det}\left[\begin{array}{ll}f_{i} & f_{j} \\ h_{i} & h_{j}\end{array}\right], i=1,2,3$.

\section{Torção e Derivada de Terceira Ordem}

Derivando $\ddot{\beta}=(0, \ddot{y}, \ddot{z})$, obtemos uma primeira expressão para o vetor $\dddot{\beta}$ :

$$
\dddot{\beta}=(0, \dddot{y}, \dddot{z}) \text {. }
$$

E, pela equação (2.31)

$$
\begin{aligned}
& {[\nabla f][\dddot{\beta}]=-3[\dot{\beta}]^{T} H_{1}[\ddot{\beta}]-[\dot{\beta}]^{T} \dot{H}_{1}[\dot{\beta}]} \\
& {[\nabla h][\dddot{\beta}]=-3[\dot{\beta}]^{T} H_{2}[\ddot{\beta}]-[\dot{\beta}]^{T} \dot{H}_{2}[\dot{\beta}] .}
\end{aligned}
$$

Assim,

$$
\begin{aligned}
& {\left[\begin{array}{lll}
f_{x} & f_{y} & f_{z} \\
h_{x} & h_{y} & h_{z}
\end{array}\right]\left[\begin{array}{c}
0 \\
\dddot{y} \\
\dddot{z}
\end{array}\right]=\left[\begin{array}{c}
-3[\dot{\beta}]^{T} H_{1}[\ddot{\beta}]-[\dot{\beta}]^{T} \dot{H}_{1}[\dot{\beta}] \\
-3[\dot{\beta}]^{T} H_{2}[\ddot{\beta}]-[\dot{\beta}]^{T} \dot{H}_{2}[\dot{\beta}]
\end{array}\right] } \\
& {\left[\begin{array}{c}
\dddot{y} \\
\dddot{z}
\end{array}\right]=-\frac{1}{A_{23}}\left[\begin{array}{cc}
h_{z} & -f_{z} \\
-h_{y} & f_{y}
\end{array}\right]\left[\begin{array}{l}
3[\dot{\beta}]^{T} H_{1}[\ddot{\beta}]+[\dot{\beta}]^{T} \dot{H}_{1}[\dot{\beta}] \\
3[\dot{\beta}]^{T} H_{2}[\ddot{\beta}]+[\dot{\beta}]^{T} \dot{H}_{2}[\dot{\beta}]
\end{array}\right] . }
\end{aligned}
$$

Observe que

- $[\dot{\beta}]^{T} H_{1}[\ddot{\beta}]=\left[\left(f_{x y}+\dot{y} f_{y y}+\dot{z} f_{y z}\right) \ddot{y}+\left(f_{x z}+\dot{y} f_{y z}+\dot{z} f_{z z}\right) \ddot{z}\right]$,

- $[\dot{\beta}]^{T} \dot{H}_{1}[\dot{\beta}]=\frac{1}{A_{23}^{3}}\left[A_{23}^{3} f_{x x x}-A_{13}^{3} f_{y y y}+A_{12}^{3} f_{z z z}-3\left(A_{13} A_{23}^{2} f_{x x y}-A_{12} A_{23}^{2} f_{x x z}-\right.\right.$ $\left.\left.A_{23} A_{13}^{2} f_{x y y}-A_{23} A_{12}^{2} f_{x z z}+A_{12} A_{13}^{2} f_{y y z}+A_{13} A_{12}^{2} f_{y z z}+2 A_{12} A_{13} A_{23} f_{x y z}\right)\right]$, 
de onde se tem

$$
3[\dot{\beta}]^{T} H_{1}[\ddot{\beta}]+[\dot{\beta}]^{T} \dot{H}_{1}[\dot{\beta}]=\frac{1}{A_{23}^{3}}\left(C_{11} \ddot{y}+C_{12} \ddot{z}+C_{13}\right)
$$

com

$$
\begin{aligned}
C_{11} & =3\left(A_{23}^{3} f_{x y}-A_{23}^{2} A_{13} f_{y y}+A_{23}^{2} A_{12} f_{y z}\right) \\
C_{12} & =3\left(A_{23}^{3} f_{x z}-A_{23}^{2} A_{13} f_{y z}+A_{23}^{2} A_{12} f_{z z}\right) \\
C_{13} & =A_{23}^{3} f_{x x x}-A_{13}^{3} f_{y y y}+A_{12}^{3} f_{z z z}-3\left(A_{13} A_{23}^{2} f_{x x y}-A_{12} A_{23}^{2} f_{x x z}-A_{23} A_{13}^{2} f_{x y y}\right. \\
& \left.-A_{23} A_{12}^{2} f_{x z z}-A_{12} A_{13}^{2} f_{y y z}+A_{13} A_{12}^{2} f_{y z z}+2 A_{12} A_{13} A_{23} f_{x y z}\right) .
\end{aligned}
$$

E, analogamente,

$$
3[\dot{\beta}]^{T} H_{2}[\ddot{\beta}]+[\dot{\beta}]^{T} \dot{H}_{2}[\dot{\beta}]=\frac{1}{A_{23}^{3}}\left(C_{21} \ddot{y}+C_{22} \ddot{z}+C_{23}\right),
$$

com

$$
\begin{aligned}
C_{21} & =3\left(A_{23}^{3} h_{x y}-A_{23}^{2} A_{13} h_{y y}+A_{23}^{2} A_{12} h_{y z}\right) \\
C_{22} & =3\left(A_{23}^{3} h_{x z}-A_{23}^{2} A_{13} h_{y z}+A_{23}^{2} A_{12} h_{z z}\right) \\
C_{23} & =A_{23}^{3} h_{x x x}-A_{13}^{3} h_{y y y}+A_{12}^{3} h_{z z z}-3\left(A_{13} A_{23}^{2} h_{x x y}-A_{12} A_{23}^{2} h_{x x z}-A_{23} A_{13}^{2} h_{x y y}\right. \\
& \left.-A_{23} A_{12}^{2} h_{x z z}-A_{12} A_{13}^{2} h_{y y z}+A_{13} A_{12}^{2} h_{y z z}+2 A_{12} A_{13} A_{23} h_{x y z}\right) .
\end{aligned}
$$

Daí, temos que

$$
\begin{aligned}
{\left[\begin{array}{c}
\dddot{y} \\
\dddot{z}
\end{array}\right] } & =-\frac{1}{A_{23}^{4}}\left[\begin{array}{rr}
h_{z} & -f_{z} \\
-h_{y} & f_{y}
\end{array}\right]\left[\begin{array}{c}
C_{11} \ddot{y}+C_{12} \ddot{z}+C_{13} \\
C_{21} \ddot{y}+C_{22} \ddot{z}+C_{23}
\end{array}\right] \\
& =-A_{23}^{-4}\left[\begin{array}{c}
h_{z}\left(C_{11} \ddot{y}+C_{12} \ddot{z}+C_{13}\right)-f_{z}\left(C_{21} \ddot{y}+C_{22} \ddot{z}+C_{23}\right) \\
-h_{y}\left(C_{11} \ddot{y}+C_{12} \ddot{z}+C_{13}\right)+f_{y}\left(C_{21} \ddot{y}+C_{22} \ddot{z}+C_{23}\right)
\end{array}\right] \\
& =-A_{23}^{-4}\left[\begin{array}{c}
\left(h_{z} C_{11}-f_{z} C_{21}\right) \ddot{y}+\left(h_{z} C_{12}-f_{z} C_{22}\right) \ddot{z}+\left(h_{z} C_{13}-f_{z} C_{23}\right) \\
\left(f_{y} C_{21}-h_{y} C_{11}\right) \ddot{y}+\left(f_{y} C_{22}-h_{y} C_{12}\right) \ddot{z}+\left(f_{y} C_{23}-h_{y} C_{13}\right)
\end{array}\right] .
\end{aligned}
$$

Portanto,

$$
\begin{aligned}
& \dddot{y}=A_{23}^{-4}\left[\left(f_{z} C_{21}-h_{z} C_{11}\right) \ddot{y}+\left(f_{z} C_{22}-h_{z} C_{12}\right) \ddot{z}+\left(f_{z} C_{23}-h_{z} C_{13}\right)\right] \\
& \dddot{z}=-A_{23}^{-4}\left[\left(f_{y} C_{21}-h_{y} C_{11}\right) \ddot{y}+\left(f_{y} C_{22}-h_{y} C_{12}\right) \ddot{z}+\left(f_{y} C_{23}-h_{y} C_{13}\right)\right],
\end{aligned}
$$


o que nos fornece uma expressão para $\dddot{\beta}=(0, \dddot{y}, \dddot{z})$. Para calcular a torção, lembremos de (1.2) que

$$
\tau=\frac{\langle\dot{\beta} \wedge \ddot{\beta}, \dddot{\beta}\rangle}{\|\dot{\beta} \wedge \ddot{\beta}\|^{2}}
$$

Além disso,

- $\dot{\beta} \wedge \ddot{\beta}=(\dot{y} \ddot{z}-\dot{z} \ddot{y},-\ddot{z}, \ddot{y})$,

- $\langle\dot{\beta} \wedge \ddot{\beta}, \dddot{\beta}\rangle=\dddot{y} \dddot{z}-\ddot{z} \dddot{y}$,

- $\|\dot{\beta} \wedge \ddot{\beta}\|^{2}=\kappa^{2}\|\beta\|^{6}$.

Por conseguinte,

$$
\tau=\frac{\dddot{y} \dddot{z}-\ddot{z} \dddot{y}}{\kappa^{2}\|\dot{\beta}\|^{6}}
$$

Dessa forma, substituindo os valores de $\kappa$ e $\|\beta\|^{6}$ (ver Teorema 2.6 e equação (2.43)) na equação (2.45), temos demonstrado o teorema a seguir.

Teorema 2.7. A torção da curva de interseção transversal $\beta$ entre as superfícies implícitas $f$ e hé dada por

$$
\tau=\frac{A_{23}^{2}(\dddot{y} \dddot{z}-\ddot{z} \dddot{y})}{\left(A_{12}^{2}+A_{23}^{2}\right)\left(\ddot{y}^{2}\right)+2 A_{12} A_{13} \ddot{y} \ddot{z}+\left(A_{13}^{2}+A_{23}^{2}\right)\left(\ddot{z}^{2}\right)^{\prime}},
$$

em que os coeficientes $A_{i j}$ são definidos em (2.36).

Os corolários que serão enunciados a seguir são consequências dos Teoremas 2.6 e 2.7 ou de suas demonstrações. Estes resultados fornecem condições necessárias e suficientes para que a curva de interseção seja uma reta, curva plana, hélice, hélice circular ou um círculo.

Corolário 2.2. A curva $\beta$ é uma reta se, e somente se, valem as igualdades

$$
\left\{\begin{array}{l}
B_{311} A_{23}^{2}+B_{322} A_{13}^{2}+B_{333} A_{12}^{2}=2\left(B_{312} A_{13} A_{23}+B_{323} A_{12} A_{13}-B_{313} A_{12} A_{23}\right) \\
B_{211} A_{23}^{2}+B_{222} A_{13}^{2}+B_{233} A_{12}^{2}=2\left(B_{212} A_{13} A_{23}+B_{223} A_{12} A_{13}-B_{213} A_{12} A_{23}\right) .
\end{array}\right.
$$

Demonstração. De fato, $\beta$ é uma reta se, e somente se, $\kappa=0$, o que, por (2.42), equivale a $\dot{\beta} \wedge \ddot{\beta}=0$. Lembre, de (2.43), que $\dot{\beta} \wedge \ddot{\beta}=-\frac{1}{A_{23}}\left(A_{12} \ddot{y}+A_{13} \ddot{z}, A_{23} \ddot{z},-A_{23} \ddot{y}\right)$. Portanto,

$$
\kappa=0 \Leftrightarrow \ddot{y}=\ddot{z}=0,
$$

o que, por (2.40) e (2.41), nos leva ao resultado. 
Corolário 2.3. A curva $\beta$ é uma curva plana se, e somente se, a seguinte igualdade é verificada

$$
\begin{aligned}
& \left(C_{21} f_{y}-C_{11} h_{y}\right)(\ddot{y})^{2}+\left(C_{22} f_{z}-C_{12} h_{z}\right)(\ddot{z})^{2}+\left(C_{23} f_{y}-C_{13} h_{y}\right) \ddot{y}+\left(C_{23} f_{z}-C_{13} h_{z}\right) \ddot{z}+ \\
& \left(C_{21} f_{z}+C_{22} f_{y}-C_{11} h_{z}-C_{12} h_{y}\right) \ddot{y} \ddot{z}=0 .
\end{aligned}
$$

Demonstração. De fato, $\beta$ é curva plana se, e somente se, $\tau=0$. Pela equação (2.45), temos que

$$
\tau=0 \Leftrightarrow \ddot{y} \dddot{z}-\ddot{z} \dddot{y}=0 \text {. }
$$

Ora, note que substituindo em $\dddot{y} \dddot{z}-\ddot{z} \dddot{y}$ os valores de $\dddot{y}$ e $\dddot{z}$ dados em (2.44), tem-se

$$
\begin{aligned}
\dddot{y} \dddot{z}-\ddot{z} \dddot{y}= & -A_{23}^{-4}\left[\left(C_{21} f_{y}-C_{11} h_{y}\right)(\ddot{y})^{2}+\left(C_{22} f_{z}-C_{12} h_{z}\right)(\ddot{z})^{2}+\left(C_{23} f_{y}-C_{13} h_{y}\right) \ddot{y}\right. \\
& \left.+\left(C_{23} f_{z}-C_{13} h_{z}\right) \ddot{z}+\left(C_{21} f_{z}+C_{22} f_{y}-C_{11} h_{z}-C_{12} h_{y}\right) \ddot{y} \ddot{z}\right] .
\end{aligned}
$$

Daí, segue o resultado.

Corolário 2.4. Uma condição necessária e suficiente para $\beta$ ser uma hélice é dada por

$$
\begin{aligned}
& \left(A_{12}^{2}+A_{13}^{2}+A_{23}^{2}\right)^{-3}\left[\left(A_{12}^{2}+A_{23}^{2}\right)(\ddot{y})^{2}+2 A_{12} A_{13} \ddot{y} \ddot{z}+\left(A_{13}^{2}+A_{23}^{2}\right)(\ddot{z})^{2}\right]^{3} \\
& =c A_{23}^{-8}\left[\left(C_{21} f_{y}-C_{11} h_{y}\right)(\ddot{y})^{2}+\left(C_{22} f_{z}-C_{12} h_{z}\right)(\ddot{z})^{2}+\left(C_{23} f_{y}-C_{13} h_{y}\right) \ddot{y}\right. \\
& \left.+\left(C_{23} f_{z}-C_{13} h_{z}\right) \ddot{z}+\left(C_{21} f_{z}+C_{22} f_{y}-C_{11} h_{z}-C_{12} h_{y}\right) \ddot{y} \ddot{z}\right],
\end{aligned}
$$

para alguma constante $c$.

Demonstração. Sabe-se que $\beta$ é uma hélice se, e somente se, $\frac{\kappa}{\tau}=c_{1}$, para alguma constante $c_{1}$. Veja que

$$
\frac{\kappa}{\tau}=c_{1} \Leftrightarrow \kappa^{2}=c_{1}^{2} \tau^{2} \Leftrightarrow \frac{\left[\left(A_{12}^{2}+A_{23}^{2}\right)(\ddot{y})^{2}+2 A_{12} A_{13} \ddot{y} \ddot{z}+\left(A_{13}^{2}+A_{23}^{2}\right)(\ddot{z})^{2}\right]^{3}}{\left(A_{23}^{2}+A_{13}^{2}+A_{12}^{2}\right)^{3}}=c_{1}^{2}(\dddot{y} \dddot{z}-\ddot{z} \dddot{y})^{2} .
$$

Substituindo o valor de $\dddot{y} \dddot{z}-\ddot{z} \dddot{y}$ dado em (2.46) e definindo $c:=c_{1}^{2}$, segue o resultado.

Corolário 2.5. Uma condição necessária e suficiente para $\beta$ ser uma hélice circular é dada por

$$
\left\{\begin{array}{l}
a\left(A_{12}^{2}+A_{13}^{2}+A_{23}^{2}\right)^{3}=A_{23}^{4}\left(A_{12}^{2}+A_{23}^{2}\right)(\ddot{y})^{2}+2 A_{12} A_{13} \ddot{y} \ddot{z}+\left(A_{13}^{2}+A_{23}^{2}\right)(\ddot{z})^{2} \\
b A_{23}^{2}\left[\left(A_{12}^{2}+A_{23}^{2}\right)(\ddot{y})^{2}+2 A_{12} A_{13} \ddot{y} \ddot{z}+\left(A_{13}^{2}+A_{23}^{2}\right)(\ddot{z})^{2}\right]=\left[\left(C_{22} f_{z}-C_{12} h_{z}\right)(\ddot{z})^{2}+\left(C_{21} f_{y}\right.\right. \\
\left.\left.-C_{11} h_{y}\right)(\ddot{y})^{2}+\left(C_{23} f_{z}-C_{13} h_{z}\right) \ddot{z}+\left(C_{23} f_{y}-C_{13} h_{y}\right) \ddot{y}+\left(C_{21} f_{z}+C_{22} f_{y}-C_{11} h_{z}-C_{12} h_{y}\right) \ddot{y} \ddot{z}\right],
\end{array}\right.
$$

para algumas constantes $a e b$. 
Demonstração. Sabe-se que uma curva é uma hélice circular se, e somente se, possui curvatura e torção constantes. Sejam, portanto, $\kappa=a_{1}$ e $\tau=b_{1}$. Tomando-se o quadrado da curvatura $\kappa$ dada no Teorema 2.6 e fazendo $a=a_{1}$, segue a primeira igualdade do sistema acima. A segunda igualdade segue do Teorema 2.7 e da equação (2.46), fazendo-se $b=-b_{1}$.

Corolário 2.6. Uma condição necessária e suficiente para a curva $\beta$ ser um círculo é dada por

$$
\left\{\begin{array}{l}
c\left(A_{12}^{2}+A_{13}^{2}+A_{23}^{2}\right)^{3}=A_{23}^{4}\left(A_{12}^{2}+A_{23}^{2}\right)(\ddot{y})^{2}+2 A_{12} A_{13} \dddot{y} \ddot{z}+\left(A_{13}^{2}+A_{23}^{2}\right)(\ddot{z})^{2} \\
\left(C_{22} f_{z}-C_{12} h_{z}\right)(\ddot{z})^{2}+\left(C_{21} f_{y}-C_{11} h_{y}\right)(\ddot{y})^{2}+\left(C_{23} f_{z}-C_{13} h_{z}\right) \ddot{z}+\left(C_{23} f_{y}-C_{13} h_{y}\right) \ddot{y}+ \\
\left(C_{21} f_{z}+C_{22} f_{y}-C_{11} h_{z}-C_{12} h_{y}\right) \dddot{y} \ddot{z}=0,
\end{array}\right.
$$

para alguma constante $c$.

Demonstração. Sabemos que $\beta$ é um círculo se, e somente se, $\kappa=c$, para alguma constante $c$, e $\tau=0$. Assim, a demonstração segue de maneira análoga à anterior.

\subsection{Interseção Tangencial}

A seguir, faremos o estudo das curvas de interseção tangencial para os casos ParamétricaParamétrica, Paramétrica-Implícita e Implícita-Implícita. Neste caso, os vetores normais das superfícies são paralelos. As referências básicas são as mesmas descritas na Seção 2.1. Assim como fizemos para as interseções transversais, vamos descrever métodos para se obter propriedades da curva de interseção tais como vetor tangente, vetor curvatura, curvatura e torção. No caso Paramétrica-Paramétrica, é dada uma interpretação geométrica para o vetor tangente à curva de interseção num ponto $P$.

\subsubsection{Interseção entre duas Superfícies Paramétricas}

Sejam $X\left(u_{1}, v_{1}\right)$ e $Y\left(u_{2}, v_{2}\right)$ superfícies paramétricas que se intersectam tangencialmente num ponto $P$ que está sobre a curva de interseção $\alpha$. Isto significa que $N^{X} \| N^{Y}$ em $P$. Orientando as superfícies de maneira conveniente, podemos supor que $N^{X}=N^{Y}$. Assim, denotaremos $N=N^{X}=N^{Y}$. Neste caso, a equação (2.1) não é válida, e temos que encontrar novos métodos para calcular as propriedades geométricas de $\alpha$, o que faremos a partir de agora. No que segue, omitiremos o ponto $P$ por simplicidade e utilizaremos como base a referência [18]. 


\section{Vetor Tangente}

Neste caso, o vetor tangente em $P$ está sobre os planos tangentes de ambas as superfícies. Então, podemos escrever (ver equações (1.4))

$$
t=X_{u_{1}} u_{1}^{\prime}+X_{v_{1}} v_{1}^{\prime}=Y_{u_{2}} u_{2}^{\prime}+Y_{v_{2}} v_{2}^{\prime}
$$

Note que (2.47) forma um sistema de duas equações lineares com quatro variáveis $u_{1}^{\prime}, v_{1}^{\prime}$, $u_{2}^{\prime}, v_{2}^{\prime}$, visto que os vetores envolvidos não têm a componente normal.

Da equação (1.6), tem-se que $\kappa_{n}^{X}=\kappa_{n}^{Y}$, já que $N^{X}=N^{Y}=N$. Logo, de (2.5), temos que

$$
e^{X}\left(u_{1}^{\prime}\right)^{2}+2 f^{X} u_{1}^{\prime} v_{1}^{\prime}+g^{X}\left(v_{1}^{\prime}\right)^{2}=e^{Y}\left(u_{2}^{\prime}\right)^{2}+2 f^{Y} u_{2}^{\prime} v_{2}^{\prime}+g^{Y}\left(v_{2}^{\prime}\right)^{2} \text {, }
$$

onde os respectivos $e, f, g$ denotam os coeficientes da segunda forma fundamental nas superfícies $X$ e $Y$ em $P$. Em outras palavras, temos que $I I_{p}^{X}(t)=I I_{p}^{Y}(t)$, onde $t$ é o vetor tangente à curva de interseção $\alpha$ em $P=\alpha(t)$.

Escrevendo os vetores da base $\left\{X_{u_{1}}, X_{v_{1}}\right\}$ na base $\left\{Y_{u_{2}}, Y_{v_{2}}\right\}$, segue que

$$
\begin{aligned}
& X_{u_{1}}=a_{11} Y_{u_{2}}+a_{21} Y_{v_{2}} \\
& X_{v_{1}}=a_{12} Y_{u_{2}}+a_{22} Y_{v_{2}} .
\end{aligned}
$$

Logo, substituindo (2.49) em (2.47), tem-se

$$
\begin{aligned}
& u_{2}^{\prime}=a_{11} u_{1}^{\prime}+a_{12} v_{1}^{\prime} \\
& v_{2}^{\prime}=a_{21} u_{1}^{\prime}+a_{22} v_{1}^{\prime} .
\end{aligned}
$$

Para avaliar os coeficientes $a_{i j}$, toma-se o produto vetorial de (2.49) com $Y_{u_{2}}$ e $Y_{v_{2}}$ e o produto interno disso com o normal $N$, obtendo

$$
\begin{aligned}
& a_{11}=\frac{\left\langle X_{u_{1}} \wedge Y_{v_{2}}, N\right\rangle}{\left\langle Y_{u_{2}} \wedge Y_{v_{2}}, N\right\rangle}=\frac{\operatorname{det}\left(X_{u_{1}}, Y_{v_{2}}, N\right)}{\sqrt{E^{Y} G^{Y}-\left(F^{Y}\right)^{2}}} \\
& a_{12}=\frac{\left\langle X_{v_{1}} \wedge Y_{v_{2}}, N\right\rangle}{\left\langle Y_{u_{2}} \wedge Y_{v_{2}}, N\right\rangle}=\frac{\operatorname{det}\left(X_{v_{1}}, Y_{v_{2}}, N\right)}{\sqrt{E^{Y} G^{Y}-\left(F^{Y}\right)^{2}}} \\
& a_{21}=\frac{\left\langle Y_{u_{2}} \wedge X_{u_{1}}, N\right\rangle}{\left\langle Y_{u_{2}} \wedge Y_{v_{2}}, N\right\rangle}=\frac{\operatorname{det}\left(Y_{u_{2}}, X_{u_{1}}, N\right)}{\sqrt{E^{Y} G^{Y}-\left(F^{Y}\right)^{2}}} \\
& a_{22}=\frac{\left\langle Y_{u_{2}} \wedge X_{v_{1}}, N\right\rangle}{\left\langle Y_{u_{2}} \wedge Y_{v_{2}}, N\right\rangle}=\frac{\operatorname{det}\left(Y_{u_{2}}, X_{v_{1}}, N\right)}{\sqrt{E^{Y} G^{Y}-\left(F^{Y}\right)^{2}}} .
\end{aligned}
$$

Substituindo (2.50) em (2.48), temos:

$$
\begin{aligned}
e^{X}\left(u_{1}^{\prime}\right)^{2}+2 f^{X} u_{1}^{\prime} v_{1}^{\prime}+g^{X}\left(v_{1}^{\prime}\right)^{2} & =e^{Y}\left[a_{11}^{2}\left(u_{1}^{\prime}\right)^{2}+2 a_{11} a_{12} u_{1}^{\prime} v_{1}^{\prime}+a_{12}^{2}\left(v_{1}^{\prime}\right)^{2}\right] \\
& +2 f^{Y}\left[a_{11} a_{21}\left(u_{1}^{\prime}\right)^{2}+\left(a_{11} a_{22}+a_{12} a_{21}\right) u_{1}^{\prime} v_{1}^{\prime}+a_{12} a_{22}\left(v_{1}^{\prime}\right)^{2}\right] \\
& +g^{Y}\left[a_{21}^{2}\left(u_{1}^{\prime}\right)^{2}+2 a_{21} a_{22} u_{1}^{\prime} v_{1}^{\prime}+a_{22}^{2}\left(v_{1}^{\prime}\right)^{2}\right],
\end{aligned}
$$


o que implica

$$
b_{11}\left(u_{1}^{\prime}\right)^{2}+2 b_{12} u_{1}^{\prime} v_{1}^{\prime}+b_{22}\left(v_{1}^{\prime}\right)^{2}=0,
$$

onde

$$
\begin{aligned}
& b_{11}=e^{Y} a_{11}^{2}+2 f^{Y} a_{11} a_{21}+g^{Y} a_{21}^{2}-e^{X} \\
& b_{12}=e^{Y} a_{11} a_{12}+f^{Y}\left(a_{11} a_{22}+a_{12} a_{21}\right)+g^{Y} a_{21} a_{22}-f^{X} \\
& b_{22}=e^{Y} a_{12}^{2}+2 f^{Y} a_{12} a_{22}+g^{Y} a_{22}^{2}-g^{X} .
\end{aligned}
$$

Utilizamos a equação (2.52) para demonstrar o teorema que segue.

Teorema 2.8. Sejam $X$ e $Y$ superfícies paramétricas que se intersectam tangencialmente no ponto $P=$ $\alpha(t)$. Suponha que $b_{11}^{2}+b_{12}^{2}+b_{22}^{2} \neq 0$. Então, o vetor tangente a $\alpha$ no ponto $P$ é dado por

$$
\begin{aligned}
& t=\frac{\omega X_{u_{1}}+X_{v_{1}}}{\left\|\omega X_{u_{1}}+X_{v_{1}}\right\|}, \quad \text { se } b_{11} \neq 0 \\
& t=\frac{X_{u_{1}}+\mu X_{v_{1}}}{\left\|X_{u_{1}}+\mu X_{v_{1}}\right\|}, \\
& t \| X_{u_{1}} \text { ou } t \| X_{v_{1}}, \quad \text { se } b_{11}=0 \text { e } b_{22} \neq 0
\end{aligned}
$$

onde $\omega=\frac{u_{1}^{\prime}}{v_{1}^{\prime}}$ é solução da equação $b_{11} \omega^{2}+2 b_{12} \omega+b_{22}=0$ e $\mu=\frac{v_{1}^{\prime}}{u_{1}^{\prime}}$ é solução da equação $b_{22} \mu^{2}+$ $2 b_{12} \mu+b_{11}=0$, que seguem de (2.52).

Demonstração. Se $b_{11} \neq 0$, não ocorre $v_{1}^{\prime}=0$. Assim, definindo $\omega=\frac{u_{1}^{\prime}}{v_{1}^{\prime}}$, obtemos

$$
b_{11} \omega^{2}+2 b_{12} \omega+b_{22}=0 .
$$

Daí,

$$
\omega=\frac{-b_{12} \pm \sqrt{b_{12}^{2}-b_{11} b_{22}}}{b_{11}}
$$

Note que

$$
t=X_{u_{1}} u_{1}^{\prime}+X_{v_{1}} v_{1}^{\prime}=\left(\omega X_{u_{1}}+X_{v_{1}}\right) v_{1}^{\prime}
$$

Do fato de $\|t\|=1$, concluímos que $v_{1}^{\prime}=\frac{1}{\left\|\omega X_{u_{1}}+X_{v_{1}}\right\|}$. Portanto, o vetor tangente é dado por

$$
t=\frac{\omega X_{u_{1}}+X_{v_{1}}}{\left\|\omega X_{u_{1}}+X_{v_{1}}\right\|} .
$$


Agora, se $b_{11}=0$, mas $b_{22} \neq 0$, divida (2.52) por $\left(u_{1}^{\prime}\right)^{2}$ e denote $\mu=\frac{v_{1}^{\prime}}{u_{1}^{\prime}}$. Neste caso, a equação (2.52) fica

$$
b_{22} \mu^{2}+2 b_{12} \mu=0
$$

Daí,

$$
\mu=\frac{-b_{12} \pm b_{12}}{b_{22}}
$$

Assim, de maneira inteiramente análoga à anterior, tem-se

$$
t=\frac{X_{u_{1}}+\mu X_{v_{1}}}{\left\|X_{u_{1}}+\mu X_{v_{1}}\right\|}
$$

Se $b_{11}=b_{22}=0$, então, se $b_{12} \neq 0$, tem-se que $u_{1}^{\prime}=0$ ou $v_{1}^{\prime}=0$, ou seja, o vetor tangente é paralelo a um dos vetores da base $\left\{X_{u_{1}}, X_{v_{1}}\right\}$ (ver equação 2.47).

Podemos classificar os pontos de interseção entre $X$ e $Y$ de acordo com o discriminante $\Delta=4\left(b_{12}^{2}-b_{11} b_{22}\right)$ das equações (2.54) e (2.55), que definem o tangente $t$.

Caso 1. Se $\Delta<0$, como as soluções $\omega$ ou $\mu$, que definem o vetor tangente à curva de interseção $\alpha$, envolvem o termo $\sqrt{\Delta}$, chegamos a uma contradição com a hipótese inicial de existir uma curva de interseção passando por $P$. Logo, $P$ é ponto de contato isolado.

Caso 2. Se $\Delta>0$, então a equação (2.52) possui duas raízes distintas, ou seja, existem dois vetores tangentes definidos em $P$. Logo, $P$ é ponto de ramificação.

Caso 3. Se $\Delta=0 \operatorname{com} b_{11}^{2}+b_{12}^{2}+b_{22}^{2} \neq 0$, então as equações (2.54) e (2.55) possuem raiz dupla e, portanto, existe um único vetor tangente definido em $P$, o que nos diz que existe uma única curva de interseção passando por $P$.

Caso 4. Se $b_{11}=b_{12}=b_{22}=0$, então a equação (2.52) é satisfeita para todo $u_{1}^{\prime}$ e $v_{1}^{\prime}$, ou seja, pela equação (2.48), $I I_{P}^{X}(t)=I I_{P}^{Y}(t)$, para todo $t \in T_{P} X=T_{P} Y$. Logo, pela Proposição $1.3, P$ é ponto de contato de ordem $\geq 2$.

A seguir, daremos uma interpretação geométrica para o tangente $t$. Se $P$ é um ponto planar de uma das superfícies, digamos, $Y$, então $e^{Y}=f^{Y}=g^{Y}=0, b_{11}=-e^{X}, b_{12}=-f^{X}, b_{22}=$ $-g^{X}$ e a equação (2.52) pode ser analisada como anteriormente. Se $P$ é ponto planar de ambas as superfícies, então $b_{i j}=0, \forall i, j=1,2 \mathrm{e}$, como vimos no Caso $4, P$ é ponto de contato de ordem $\geq 2$. Agora, se em ambas as superfícies $P$ não é ponto planar, podemos analisar $t$ em termos das indicatrizes de Dupin de $X$ e $Y$ em $P$.

Proposição 2.1. Seja P um ponto não planar em X e em $Y$. Se o vetor tangente t está bem definido em $P$, então as indicatrizes de Dupin de X e Y em $P$ se intersectam ao longo dos vetores \pm ct, para alguma constante $c$. 
Demonstração. Sejam $\mathcal{I}^{X}$ e $\mathcal{I}^{Y}$ as indicatrizes de Dupin em $P$ de $X$ e $Y$, respectivamente. Pela equação (2.48), $I I_{P}^{X}(t)=I I_{P}^{Y}(t)=$ : $c_{0}$. Logo

$$
\frac{I I_{P}^{X}(t)}{\left|c_{0}\right|}=\frac{I I_{P}^{Y}(t)}{\left|c_{0}\right|}= \pm 1 \Rightarrow I I_{P}^{X}\left( \pm \frac{t}{\sqrt{\left|c_{0}\right|}}\right)=I I_{P}^{Y}\left( \pm \frac{t}{\sqrt{\left|c_{0}\right|}}\right)= \pm 1
$$

Defina $c=\frac{1}{\sqrt{\left|c_{0}\right|}}$. Logo, pela definição da indicatriz de Dupin, segue que $\mathcal{I}^{X}$ e $\mathcal{I}^{Y}$ se intersectam em $c t$ e $-c t$.

Escrito de outro modo, o resultado nos diz que, se as indicatrizes de Dupin não se intersectam, então não se tem vetor tangente bem definido em $P$ e, portanto, $P$ é ponto de contato isolado. Por outro lado, poderíamos nos perguntar se o fato de as indicatrizes de Dupin se intersectarem implica a existência de vetor tangente a uma curva de interseção em $P$, isto é, que $P$ não é ponto isolado. A resposta é negativa, como mostram os exemplos a seguir.

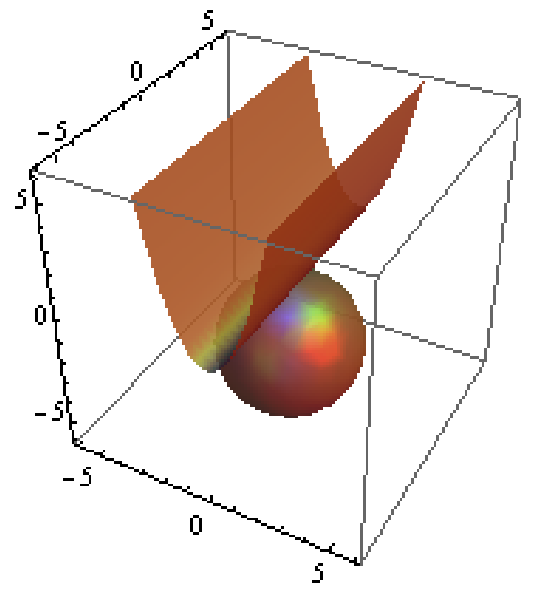

(a) Ponto de contato isolado entre uma calha parabólica e uma esfera

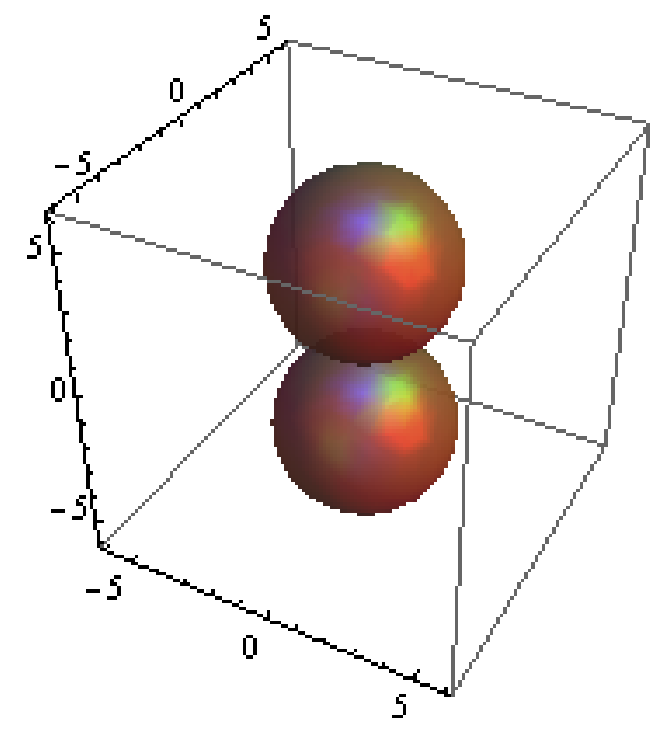

(b) Ponto de contato isolado entre duas esferas

Figura 2.1: Nestes casos, embora não se tenha curva de interseção entre as superfícies, as indicatrizes de Dupin coincidem

Exemplo 2.1. Na Figura 2.1(a), temos a interseção da calha parabólica $X(u, v)=\left(u, v, u^{2}\right)$ com a esfera $Y(\theta, \varphi)=(3 \cos \theta \cos \varphi, 3 \sin \theta, 3 \cos \theta \sin \varphi-3)$. Note que $P=(0,0,0)$ é um ponto de interseção tangencial e contato isolado. Analisando as seções normais de X e $Y$ em $P$, vemos que $\kappa_{1}^{X}=0$, que é a curvatura da reta $\beta(v)=(0, v, 0), \kappa_{2}^{X}=2$, que é a curvatura da parábola $\beta_{1}(u)=\left(u, 0, u^{2}\right)$. E, como todas as seções normais da esfera $Y$ em $P$ são grandes círculos, segue que $\kappa_{1}^{X}=\kappa_{1}^{Y}=\frac{1}{9}$. Portanto, pela expressão da indicatriz de Dupin, temos que $\mathcal{I}^{X}$ é um par de retas paralelas à direção principal $e_{2}^{X}$ e $\mathcal{I}^{Y} e^{\prime}$ 
um círculo de raio 3 centrado na origem de $T_{P} Y$, tendo elas, portanto, quatro pontos de interseção, como mostra a Figura 2.2(a).

Exemplo 2.2. Na Figura 2.1(b), temos novamente que P é ponto de contato isolado entre as esferas

$$
\begin{aligned}
X(\vartheta, \varphi) & =(3 \cos \vartheta \cos \varphi, 3 \sin \vartheta, 3 \cos \vartheta \sin \varphi-3) \\
Y(\theta, \phi) & =(3 \cos \theta \cos \phi, 3 \sin \theta, 3 \cos \theta \sin \phi+3),
\end{aligned}
$$

cujas indicatrizes de Dupin são círculos de raio 3 na origem do plano tangente comum em P e, portanto, coincidem (ver Figura 2.2(b)).

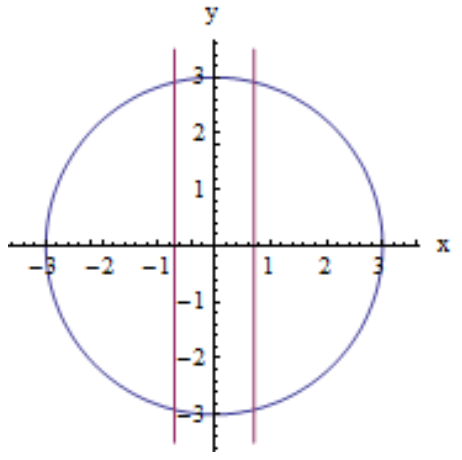

(a) Indicatrizes de Dupin da calha e da esfera de raio 3 se intersectam em 4 pontos

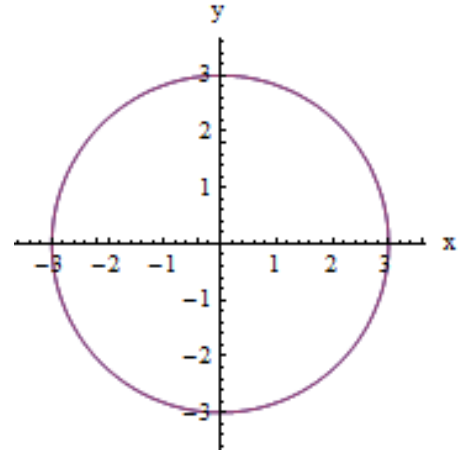

(b) Indicatrizes de Dupin de duas esferas de raio 3 se sobrepõem

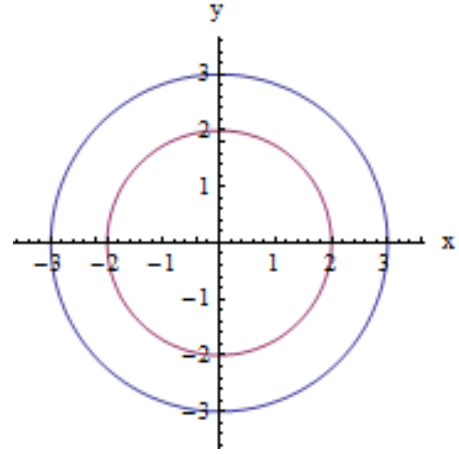

(c) Indicatrizes de Dupin das esferas de raios 3 e 2 não se intersectam

Figura 2.2: Algumas possibilidades para a interseção das indicatrizes de Dupin em um ponto de contato isolado

Observação 2.1. Seguem da Proposição 2.1 as seguintes observações:

1. Se P é ponto de contato isolado, não há vetor tangente definido neste. Logo, as indicatrizes podem não se intersectar.

2. Se há um único vetor tangente definido em $P$, então as indicatrizes de Dupin se intersectam em, pelo menos, dois pontos.

3. Se P é ponto de ramificação, há dois vetores tangentes em P. Logo, as indicatrizes de Dupin se intersectam em, no mínimo, quatro pontos.

4. Se P é ponto de contato de ordem $\geq 2$, então as segundas formas fundamentais de X e Y em $P$ coincidem e, portanto, as indicatrizes de Dupin se sobrepõem.

A seguir, daremos alguns exemplos de cada situação descrita acima, onde $\mathcal{I}^{X}$ denotará a indicatriz de Dupin da superfície $X$ no ponto $P$ considerado. 
1. Se $P$ é ponto de contato isolado, as indicatrizes de Dupin podem se intersectar ou não. Como um primeiro exemplo, considere as esferas

$$
\begin{aligned}
& X(\vartheta, \varphi)=(3 \cos \vartheta \cos \varphi, 3 \sin \vartheta, 3 \cos \vartheta \sin \varphi-3) \\
& Y(\theta, \phi)=(2 \cos \theta \cos \phi, 2 \sin \theta, 2 \cos \theta \sin \phi+2)
\end{aligned}
$$

de raios 3 e 2, respectivamente, as quais têm um ponto de contato isolado em $P=(0,0,0)$. Observe que as indicatrizes de Dupin são círculos centrados na origem do plano tangente em $P$, de raios 3 e 2, os quais não se intersectam. Por outro lado, a Figura 2.1 nos mostra exemplos que, como discutidos anteriormente, são casos onde as indicatrizes se intersectam em quatro pontos ou coincidem. A Figura 2.2 mostra estas indicatrizes.

2. Se $P$ está sobre uma (única) curva de interseção, as indicatrizes de Dupin se intersectam em pelo menos dois pontos, podendo se intersectar em mais pontos. Exemplos disto são:

(a) Considere a esfera e o cilindro, parametrizados, respectivamente, por

$$
\begin{aligned}
& X(\theta, \varphi)=(\cos \theta \cos \varphi, \sin \theta, \cos \theta \sin \varphi) \\
& Y(u, v)=(\cos u, \sin u, v) .
\end{aligned}
$$

Observe que $X$ e $Y$ se intersectam tangencialmente ao longo do círculo $x^{2}+y^{2}=1$. Considerando $P=(0,1,0) \in X \cap Y$, veja que $\mathcal{I}^{X}$ é um círculo de raio 1 no plano $T_{P} X=T_{P} Y$ centrado na origem, já que as seções normais de $X$ em $P$ são os círculos máximos, que têm curvatura 1 , ou seja, as curvaturas principais de $X$ são $\kappa_{1}^{X}=\kappa_{2}^{X}=$ 1. Analogamente, olhando para as seções normais do cilindro em $P$, podemos ver que $\kappa_{1}^{Y}=0, \kappa_{2}^{Y}=1$, sendo as direções principais $e_{1}$ e $e_{2}$ paralelas aos eixos $O z$ e $O x$, respectivamente. Portanto, $\mathcal{I}^{Y}$ é um par de retas paralelas à direção $e_{2}$. Logo, $\mathcal{I}^{X} \mathrm{e}$ $\mathcal{I}^{Y}$ se intersectam em dois pontos, a saber, $(-1,0),(1,0) \in T_{P} X$ (ver Figura 2.4(a)).

(b) Considere a interseção dos toros

$$
\begin{aligned}
& (z-1)^{2}+\left(\sqrt{x^{2}+y^{2}}-1\right)^{2}=1 \\
& (z+1)^{2}+\left(\sqrt{x^{2}+y^{2}}-1\right)^{2}=1
\end{aligned}
$$

os quais podem ser parametrizados, respectivamente, por

$$
\begin{aligned}
& X(u, v)=(\cos u-v \sin u, \sin u+v \cos u, v-1) \\
& Y(u, v)=(v+1, \cos u-v \sin u, \sin u+v \cos u),
\end{aligned}
$$

e se intersectam ao longo de paralelos, como mostra a Figura 2.3. 

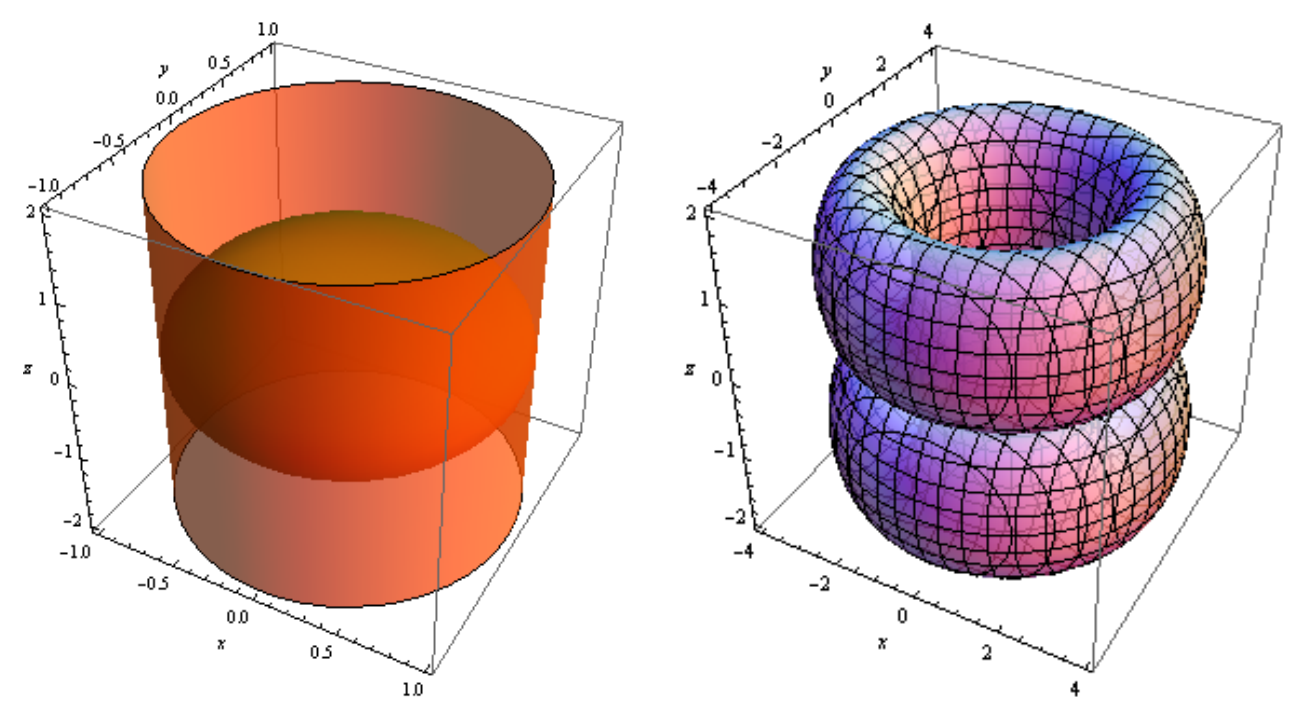

Figura 2.3: Interseção da esfera com o cilindro e de dois toros

Fixe um ponto $P$ na interseção. Sabemos que $P$ é parabólico do ponto de vista das duas superfícies. Logo, $\mathcal{I}^{X}$ e $\mathcal{I}^{Y}$ são pares de retas, paralelos às direções principais $e_{2}^{X}$ e $e_{2}^{Y}$, respectivamente. Note que $e_{2}^{X}$ e $e_{2}^{Y}$ são ambos paralelos ao eixo $O x$. Logo, como $\kappa_{2}^{X}=\kappa_{2}^{Y}=1$, as indicatrizes coincidem.

A Figura 2.4 mostra os pares de indicatrizes dos casos $(a)$ e $(b)$.

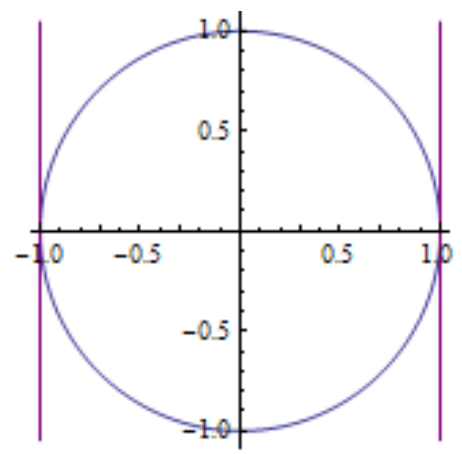

(a) Indicatrizes de Dupin da esfera e do cilindro se intersectam em dois pontos

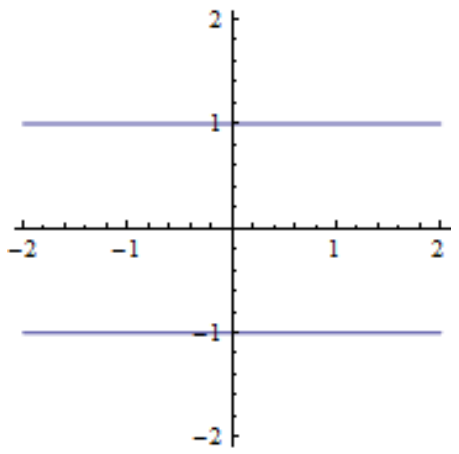

(b) Indicatrizes de Dupin dos toros coincidem

Figura 2.4: Algumas possibilidades para a interseção das indicatrizes de Dupin em um ponto sobre uma (única) curva de interseção

3. Daremos um exemplo onde as indicatrizes de Dupin em um ponto de ramificação se intersectam em quatro pontos e outro exemplo onde elas coincidem.

(a) Considere os cilindros de raio $1, X(u, v)=(\cos u, \sin u, v)$ e $Y(\theta, \varphi)=(\varphi, \cos \theta, \sin \theta)$, 
os quais têm $P=(0,1,0)$ como ponto de ramificação. Sabemos que $P$ é um ponto parabólico de $X$ e $Y$. Logo, cada indicatriz de Dupin é um par de retas paralelas às direções principais $e_{2}^{X}$ e $e_{2}^{Y}$, respectivamente. Pode-se ver que $e_{1}^{X} \| O x$ e $e_{2}^{X} \| O z$. Para $Y$, tem-se que $e_{1}^{Y} \| O z$ e $e_{2}^{Y} \| O x$. Daí, observando a discussão sobre indicatriz de Dupin feita na Seção 1.1.2, segue que $\mathcal{I}^{X}$ é um par de retas paralelas a $e_{2}^{X}$ e $\mathcal{I}^{Y}$ é um par de retas paralelas a $e_{2}^{Y}$. Logo, são ortogonais entre si, como ilustra a figura 2.6(a), e se intersectam em exatamente quatro pontos.

(b) Para ilustrar que $\mathcal{I}^{X}$ e $\mathcal{I}^{Y}$ podem se intersectar em mais de quatro pontos num ponto de ramificação, considere os hiperboloides de uma folha

$$
\begin{array}{r}
x^{2}+y^{2}-z^{2}-1=0 \\
-x^{2}+y^{2}+z^{2}-1=0,
\end{array}
$$

parametrizados por

$$
\begin{aligned}
& X(u, v)=(\cos u-v \sin u, \sin u+v \cos u, v) \\
& Y(u, v)=(-v, \sin u+v \cos u, \cos u-v \sin u) .
\end{aligned}
$$
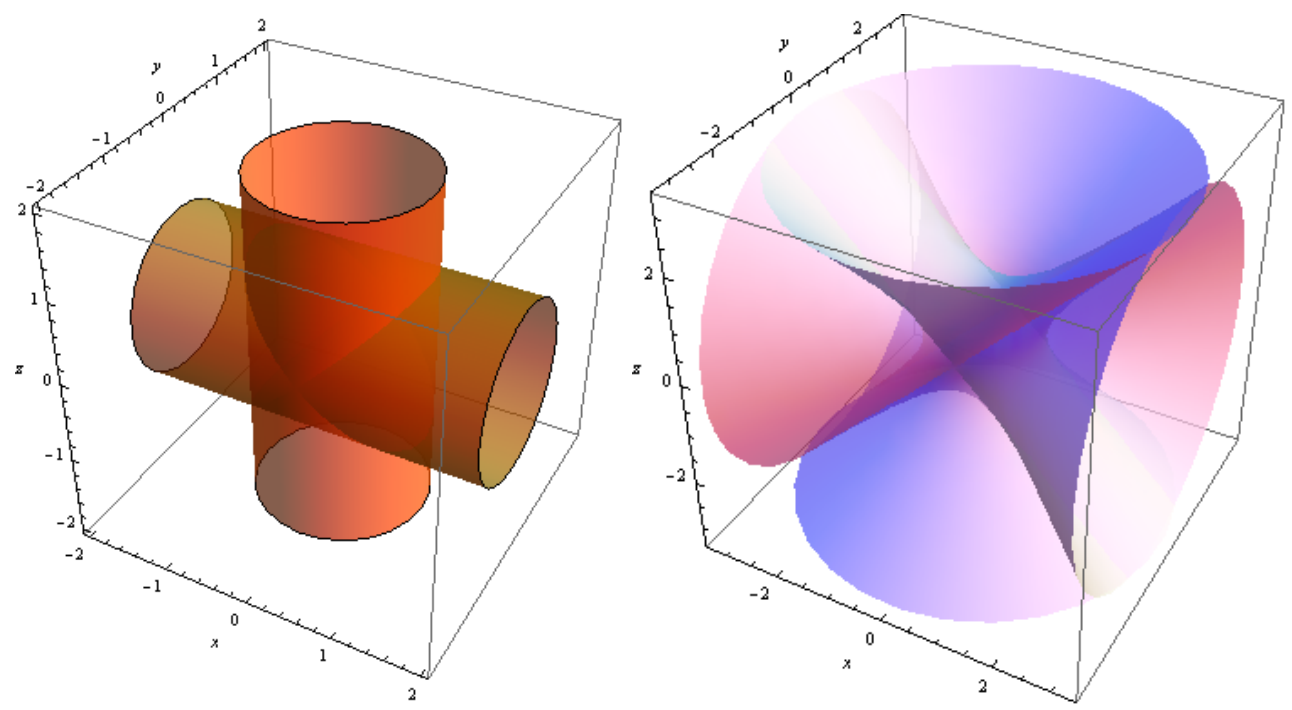

Figura 2.5: Interseção de dois cilindros e de dois hiperboloides de uma folha

Podemos verificar que $P=(0,1,0)$ é um ponto de ramificação (veja a Seção 4.1 ). Observamos, por (1.10), que as curvaturas principais de $X$ e $Y$ satisfazem

$$
\kappa_{1}^{X}=\kappa_{1}^{Y}=-1, \quad \kappa_{2}^{X}=\kappa_{2}^{Y}=1 .
$$

Portanto, sendo a indicatriz de Dupin a cônica $\kappa_{1} \xi^{2}+\kappa_{2} \eta^{2}= \pm 1$ formada pelos vetores $\omega=\xi e_{1}+\eta e_{2} \in T_{P} X=T_{P} Y$, segue que cada indicatriz $\mathcal{I}^{X}$ e $\mathcal{I}^{Y}$ é um par de hipérboles. Como $e_{2}^{X} \perp e_{2}^{Y}$, concluímos que $\mathcal{I}^{X}$ e $\mathcal{I}^{Y}$ se sobrepõem. 


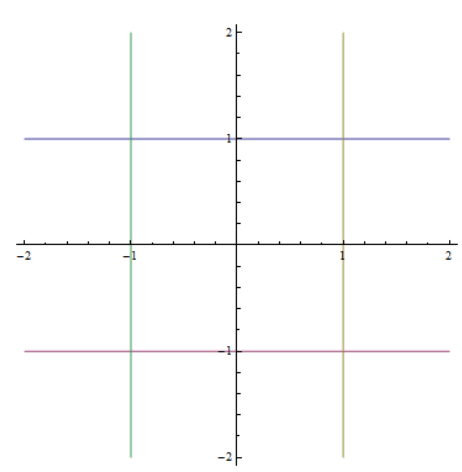

(a) Indicatrizes de Dupin de dois cilindros se intersectam em quatro pontos

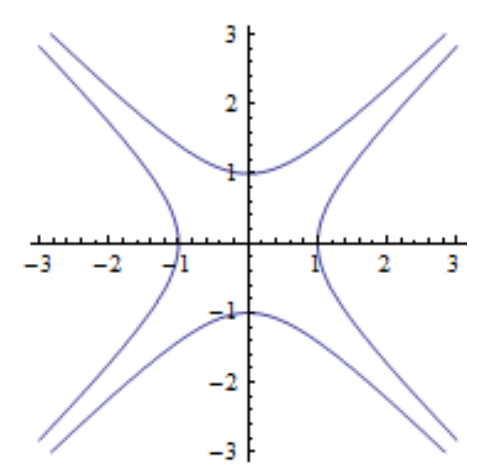

(b) Indicatrizes de Dupin dos hiperboloides de uma folha coincidem

Figura 2.6: Algumas possibilidades para a interseção das indicatrizes de Dupin em um ponto de ramificação

4. Se $P$ é um ponto de contato de ordem $\geq 2$ de $X$ e $Y$, as indicatrizes $\mathcal{I}^{X}$ e $\mathcal{I}^{Y}$ se sobrepõem. Para ilustrar esta situação, consideremos a esfera $x^{2}+y^{2}+(z-1)^{2}=1$ e a superfície paramétrica $Y(u, v)=\left(u, v, \frac{u^{2}+v^{2}}{2\left(1-u^{2}-v^{2}\right)^{\frac{3}{2}}}\right), 1-u^{2}-v^{2} \neq 0$. A superfície $Y$ é conhecida como o paraboloide osculador da esfera $S^{2}$ no ponto $P=(0,0,0)$.

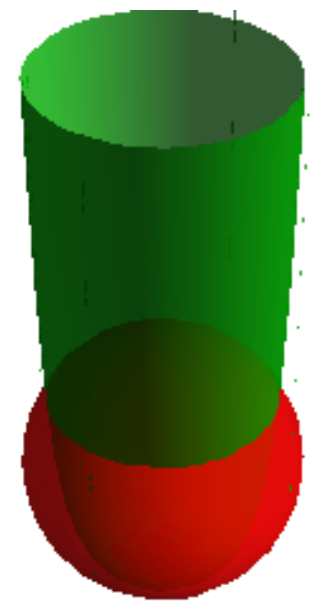

(a) Interseção da esfera $\mathrm{S}^{2}$ com o seu paraboloide osculador

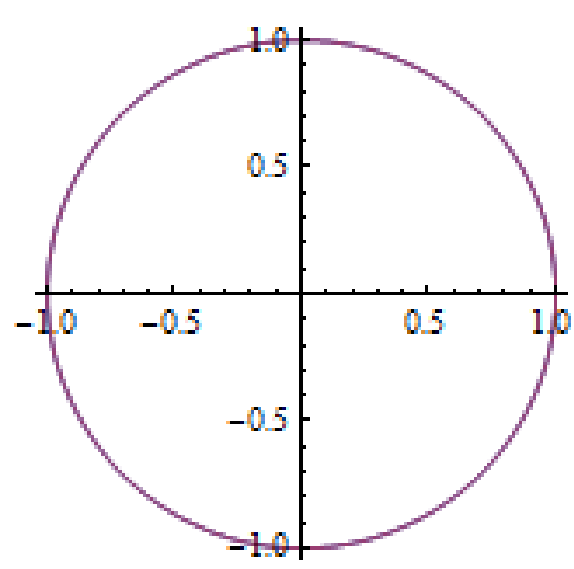

(b) A indicatrizes $\mathcal{I}^{X}$ e $\mathcal{I}^{Y}$ coincidem

Parametrize uma vizinhança de $P$ na esfera por $X(u, v)=\left(u, v, 1-\sqrt{1-u^{2}-v^{2}}\right)$.

Fazendo alguns cálculos, observamos que

$$
\begin{aligned}
& X_{u}(P)=(1,0,0)=Y_{u}(P), \quad X_{v}(P)=(0,1,0)=Y_{v}(P) \\
& X_{u u}(P)=(0,0,1)=Y_{u u}(P), \quad X_{u v}(P)=(0,0,0)=Y_{u v}(P), \quad X_{v v}(P)=(0,0,1)=Y_{v v}(P) .
\end{aligned}
$$


Como os normais são iguais, então os coeficientes da segunda forma fundamental de $X$ e $Y$ coincidem. Logo, pela Proposição 1.3, $P$ é ponto de contato de ordem $\geq 2$ destas superfícies.

Temos ainda, tanto para $X$ quanto para $Y$, que $N(P)=(0,0,1)$ e, avaliados no ponto $P$, os coeficientes da primeira e segunda formas fundamentais são

$$
E=1, F=0, G=1, e=1, f=0, g=1 \text {. }
$$

Portanto, $H=\frac{1}{2} \frac{e G-2 f F+g E}{E G-F^{2}}=1, K=\frac{e g-f^{2}}{E G-F^{2}}=1$, o que implica

$$
\kappa_{1}=\kappa_{2}=H \pm \sqrt{H^{2}-K}=1 .
$$

Ou seja, as indicatrizes $\mathcal{I}^{X}$ e $\mathcal{I}^{Y}$ se sobrepõem e são iguais a um círculo unitário centrado na origem de $T_{P} X=T_{P} Y$.

\section{Curvatura e Vetor Curvatura}

Trataremos agora da curvatura e do vetor curvatura da curva de interseção $\alpha$, lembrando que $\alpha$ é uma curva p.c.a. Primeiramente, calcularemos o vetor curvatura. Pelas equações (1.4) e, do fato de $\alpha$ estar sobre ambas as superfícies $X$ e $Y$, tem-se que

$$
\begin{aligned}
\vec{K} & =X_{u_{1} u_{1}}\left(u_{1}^{\prime}\right)^{2}+2 X_{u_{1} v_{1}} u_{1}^{\prime} v_{1}^{\prime}+X_{v_{1} v_{1}}\left(v_{1}^{\prime}\right)^{2}+X_{u_{1}} u_{1}^{\prime \prime}+X_{v_{1}} v_{1}^{\prime \prime} \\
& =Y_{u_{2} u_{2}}\left(u_{2}^{\prime}\right)^{2}+2 Y_{u_{2} v_{2}} u_{2}^{\prime} v_{2}^{\prime}+Y_{v_{2} v_{2}}\left(v_{2}^{\prime}\right)^{2}+Y_{u_{2}} u_{2}^{\prime \prime}+Y_{v_{2}} v_{2}^{\prime \prime} .
\end{aligned}
$$

Reescrevendo esta igualdade:

$$
X_{u_{1}} u_{1}^{\prime \prime}+X_{v_{1}} v_{1}^{\prime \prime}=Y_{u_{2}} u_{2}^{\prime \prime}+Y_{v_{2}} v_{2}^{\prime \prime}+\Lambda
$$

onde

$$
\Lambda=-X_{u_{1} u_{1}}\left(u_{1}^{\prime}\right)^{2}-2 X_{u_{1} v_{1}} u_{1}^{\prime} v_{1}^{\prime}-X_{v_{1} v_{1}}\left(v_{1}^{\prime}\right)^{2}+Y_{u_{2} u_{2}}\left(u_{2}^{\prime}\right)^{2}+2 Y_{u_{2} v_{2}} u_{2}^{\prime} v_{2}^{\prime}+Y_{v_{2} v_{2}}\left(v_{2}^{\prime}\right)^{2} .
$$

Tomando o produto vetorial de (2.57) por $Y_{u_{2}}$ e $Y_{v_{2}}$ e, em seguida, o produto interno por $N$, obtemos

$$
\begin{aligned}
& \left\langle Y_{u_{2}} \wedge X_{u_{1}}, N\right\rangle u_{1}^{\prime \prime}+\left\langle Y_{u_{2}} \wedge X_{v_{1}}, N\right\rangle v_{1}^{\prime \prime}=\left\langle Y_{u_{2}} \wedge Y_{v_{2}}, N\right\rangle v_{2}^{\prime \prime}+\left\langle Y_{u_{2}} \wedge \Lambda, N\right\rangle \\
& \left\langle X_{u_{1}} \wedge Y_{v_{2}}, N\right\rangle u_{1}^{\prime \prime}+\left\langle X_{v_{1}} \wedge Y_{v_{2}}, N\right\rangle v_{1}^{\prime \prime}=\left\langle Y_{u_{2}} \wedge Y_{v_{2}}, N\right\rangle u_{2}^{\prime \prime}+\left\langle\Lambda \wedge Y_{v_{2}}, N\right\rangle .
\end{aligned}
$$

Denotando

$$
a_{13}=-\frac{\left\langle\Lambda \wedge Y_{v_{2}}, N\right\rangle}{\left\langle Y_{u_{2}} \wedge Y_{v_{2}}, N\right\rangle}=-\frac{\operatorname{det}\left(\Lambda, Y_{v_{2}}, N\right)}{\sqrt{E^{Y} G^{Y}-\left(F^{Y}\right)^{2}}}, \quad a_{23}=-\frac{\left\langle Y_{u_{2}} \wedge \Lambda, N\right\rangle}{\left\langle Y_{u_{2}} \wedge Y_{v_{2}}, N\right\rangle}=-\frac{\operatorname{det}\left(Y_{u_{2}}, \Lambda, N\right)}{\sqrt{E^{Y} G^{Y}-\left(F^{Y}\right)^{2}}}
$$


e $a_{i j}(i=1,2)$ como em (2.51), segue que

$$
\begin{aligned}
& u_{2}^{\prime \prime}=a_{11} u_{1}^{\prime \prime}+a_{12} v_{1}^{\prime \prime}+a_{13} \\
& v_{2}^{\prime \prime}=a_{21} u_{1}^{\prime \prime}+a_{22} v_{1}^{\prime \prime}+a_{23},
\end{aligned}
$$

o que define $u_{2}^{\prime \prime}$ e $v_{2}^{\prime \prime}$ em função de $u_{1}^{\prime \prime}$ e $v_{1}^{\prime \prime}$. Precisamos encontrar estas duas últimas variáveis. Para isto, usaremos a terceira equação do sistema (1.4) e, novamente, o fato de $\alpha$ estar sobre ambas as superfícies $X$ e $Y$. Assim, projetando $\alpha^{\prime \prime \prime}$ sobre o normal comum a $X$ e a $Y$ e denotando isto por $\lambda_{n}^{X}$ e $\lambda_{n}^{Y}$ e fazendo $\lambda_{n}^{X}=\lambda_{n}^{Y}$, segue que

$$
\begin{aligned}
& 3\left[e^{X} u_{1}^{\prime} u_{1}^{\prime \prime}+f^{X}\left(u_{1}^{\prime \prime} v_{1}^{\prime}+u_{1}^{\prime} v_{1}^{\prime \prime}\right)+g^{X} v_{1}^{\prime} v_{1}^{\prime \prime}\right]+\tilde{\Lambda}^{X} \\
& =3\left[e^{Y} u_{2}^{\prime} u_{2}^{\prime \prime}+f^{Y}\left(u_{2}^{\prime \prime} v_{2}^{\prime}+u_{2}^{\prime} v_{2}^{\prime \prime}\right)+g^{Y} v_{2}^{\prime} v_{2}^{\prime \prime}\right]+\tilde{\Lambda}^{Y},
\end{aligned}
$$

onde os coeficientes $\tilde{\Lambda}^{X}$ e $\tilde{\Lambda}^{Y}$ são dados por (2.11). Substituindo as expressões de $u_{2}^{\prime \prime}$ e $v_{2}^{\prime \prime}$ calculadas em (2.60), tem-se

$$
\begin{aligned}
& 3\left(e^{X} u_{1}^{\prime}+f^{X} v_{1}^{\prime}-e^{Y} u_{2}^{\prime} a_{11}-f^{Y}\left(v_{2}^{\prime} a_{11}+u_{2}^{\prime} a_{21}\right)-g^{Y} v_{2}^{\prime} a_{21}\right) u_{1}^{\prime \prime}+ \\
& 3\left(f^{X} u_{1}^{\prime}+g^{X} v_{1}^{\prime}-e^{Y} u_{2}^{\prime} a_{12}-f^{Y}\left(v_{2}^{\prime} a_{12}+u_{2}^{\prime} a_{22}\right)-g^{Y} v_{2}^{\prime} a_{22}\right) v_{1}^{\prime \prime}- \\
& 3\left(e^{Y} u_{2}^{\prime} a_{13}+f^{Y}\left(v_{2}^{\prime} a_{13}+u_{2}^{\prime} a_{23}\right)+g^{Y} v_{2}^{\prime} a_{23}\right)+\tilde{\Lambda}^{X}-\tilde{\Lambda}^{Y}=0 .
\end{aligned}
$$

Outra equação envolvendo $u_{1}^{\prime \prime}$ e $v_{1}^{\prime \prime}$ é obtida de $\langle\vec{K}, t\rangle=0$. Assim, pela segunda equação do sistema (1.4),

$$
\left\langle X_{u_{1} u_{1}}, t\right\rangle\left(u_{1}^{\prime}\right)^{2}+2\left\langle X_{u_{1} v_{1}}, t\right\rangle u_{1}^{\prime} v_{1}^{\prime}+\left\langle X_{v_{1} v_{1}}, t\right\rangle\left(v_{1}^{\prime}\right)^{2}+\left\langle X_{u_{1}}, t\right\rangle u_{1}^{\prime \prime}+\left\langle X_{v_{1}}, t\right\rangle v_{1}^{\prime \prime}=0 .
$$

Dessa forma, obtemos um sistema de equações lineares nas variáveis $u_{1}^{\prime \prime}$ e $v_{1}^{\prime \prime}$. Resolvendo-o, podemos substituir os valores de $u_{1}^{\prime \prime}$ e $v_{1}^{\prime \prime}$ em (2.56) e, então, obter o vetor curvatura $\vec{K}$ e em seguida a curvatura $\kappa=\sqrt{\langle\vec{K}, \vec{K}\rangle}$.

\section{Torção e Derivada de Ordem Superior}

O vetor $\alpha^{(m)}, m \geq 3$, pode ser calculado de maneira similar ao caso do vetor curvatura. Suponha que já se tenha avaliado as derivadas $\alpha^{(j)}$ para $j=1, \ldots, m-1$ e $u^{(j-1)}, v^{(j-1)}$ para $j=2, \ldots, m-1$. O algoritmo para encontrar $\alpha^{(m)}$ é o seguinte.

1. Diferencie $m$ vezes a expressão

$$
\alpha(s)=X\left(u_{1}(s), v_{1}(s)\right)=Y\left(u_{2}(s), v_{2}(s)\right) .
$$

A partir disso, pode-se expressar os parâmetros $u_{2}^{m}$ e $v_{2}^{m}$ como combinações lineares de $u_{1}^{m}$ e $v_{1}^{m}$. 
2. Diferencie

$$
\alpha(s)=X\left(u_{1}(s), v_{1}(s)\right)=Y\left(u_{2}(s), v_{2}(s)\right)
$$

$m+1$ vezes e obtenha em seguida o número $\lambda_{n}=\left\langle\alpha^{(m+1)}, N\right\rangle$. Faça $\lambda_{n}^{X}=\lambda_{n}^{Y}$, o que gera uma equação linear nas variáveis $u_{1}^{m}, v_{1}^{m}, u_{2}^{m}$ e $v_{2}^{m}$. Substitua na equação resultante os valores de $u_{2}^{m}$ e $v_{2}^{m}$ obtidos anteriormente.

3. Utilizando as equações de Frenet (1.1), escreva $\alpha^{(m)}$ na base $\{t, n, b\}$, obtendo $\alpha^{(m)}=c_{t} t+$ $c_{n} n+c_{b} b$ e, portanto, $\left\langle\alpha^{(m)}, t\right\rangle=c_{t}$, onde $c_{t}$ depende apenas de $\kappa, \tau$ e de suas derivadas e $\alpha^{(m)}$ é a derivada de ordem $m$ de $\alpha(s)=X(u(s), v(s))$. Isto gera mais uma equação nas variáveis $u_{1}^{m}$ e $v_{1}^{m}$. Resolva o sistema resultante para $u_{1}^{m}$ e $v_{1}^{m}$.

4. Substitua os resultados na expressão de $\alpha^{(m)}$ obtida no primeiro passo.

Fazendo $m=3$, obtém-se por este método o vetor $\alpha^{\prime \prime \prime}$ e a torção fica determinada por

$$
\tau=\frac{\left\langle\alpha^{\prime \prime \prime}, b\right\rangle}{\kappa}
$$

\subsubsection{Interseção entre Superfícies Paramétricas e Implícitas}

Consideremos $X(u, v)$ uma superfície paramétrica e $f(x, y, z)=0$ uma superfície implícita, ambas regulares, as quais se intersectam tangencialmente num ponto $P$ sobre a curva de interseção $\alpha$. Assim como fizemos anteriormente, vamos calcular os entes geométricos de $\alpha$, tomando como base a referência [14].

\section{Vetor Tangente}

Observe que $N^{f}= \pm N^{X}$ implica $\left\langle\alpha^{\prime \prime}, \frac{\nabla f}{\|\nabla f\|}\right\rangle= \pm\left\langle\alpha^{\prime \prime}, \frac{X_{u} \wedge X_{v}}{\left\|X_{u} \wedge X_{v}\right\|}\right\rangle$. Denotaremos

$$
A= \pm \frac{\|\nabla f\|}{\left\|X_{u} \wedge X_{v}\right\|}
$$

De (2.5), tem-se

$$
\left\langle\alpha^{\prime \prime}, N^{X}\right\rangle=e\left(u^{\prime}\right)^{2}+2 f u^{\prime} v^{\prime}+g\left(v^{\prime}\right)^{2}
$$

Além disso, pela segunda das equações (1.11),

$$
\left\langle\alpha^{\prime \prime}, N^{f}\right\rangle=\frac{f_{x} x^{\prime \prime}+f_{y} y^{\prime \prime}+f_{z} z^{\prime \prime}}{\sqrt{f_{x}^{2}+f_{y}^{2}+f_{z}^{2}}}=-\frac{f_{x x}\left(x^{\prime}\right)^{2}+f_{y y}\left(y^{\prime}\right)^{2}+f_{z z}\left(z^{\prime}\right)^{2}+2\left(f_{x y} x^{\prime} y^{\prime}+f_{x z} x^{\prime} z^{\prime}+f_{y z} y^{\prime} z^{\prime}\right)}{\sqrt{f_{x}^{2}+f_{y}^{2}+f_{z}^{2}}} .
$$


Portanto,

$$
\begin{aligned}
-A\left\|X_{u} \wedge X_{v}\right\|\left[e\left(u^{\prime}\right)^{2}+2 f u^{\prime} v^{\prime}+g\left(v^{\prime}\right)^{2}\right] & =f_{x x}\left(x^{\prime}\right)^{2}+f_{y y}\left(y^{\prime}\right)^{2}+f_{z z}\left(z^{\prime}\right)^{2} \\
& +2\left(f_{x y} x^{\prime} y^{\prime}+f_{x z} x^{\prime} z^{\prime}+f_{y z} y^{\prime} z^{\prime}\right)
\end{aligned}
$$

Derivando os valores de $x, y, z$, dados pela equação (2.14), e substituindo na equação anterior, segue que

$$
\begin{aligned}
& f_{x x}\left(X_{u}^{1} u^{\prime}+X_{v}^{1} v^{\prime}\right)^{2}+f_{y y}\left(X_{u}^{2} u^{\prime}+X_{v}^{2} v^{\prime}\right)^{2}+f_{z z}\left(X_{u}^{3} u^{\prime}+X_{v}^{3} v^{\prime}\right)^{2}+2\left[f_{x y}\left(X_{u}^{1} u^{\prime}+X_{v}^{1} v^{\prime}\right)\left(X_{u}^{2} u^{\prime}+X_{v}^{2} v^{\prime}\right)\right. \\
& \left.+f_{x z}\left(X_{u}^{1} u^{\prime}+X_{v}^{1} v^{\prime}\right)\left(X_{u}^{3} u^{\prime}+X_{v}^{3} v^{\prime}\right)+f_{y z}\left(X_{u}^{2} u^{\prime}+X_{v}^{2} v^{\prime}\right)\left(X_{u}^{3} u^{\prime}+X_{v}^{3} v^{\prime}\right)\right] \\
& =-A\left\|X_{u} \wedge X_{v}\right\|\left[e\left(u^{\prime}\right)^{2}+2 f u^{\prime} v^{\prime}+g\left(v^{\prime}\right)^{2}\right]
\end{aligned}
$$

Daí,

$$
b_{11}\left(u^{\prime}\right)^{2}+2 b_{12} u^{\prime} v^{\prime}+b_{22}\left(v^{\prime}\right)^{2}=0
$$

onde

$$
\begin{aligned}
b_{11} & =A\left\|X_{u} \wedge X_{v}\right\| e+2\left[f_{x y} X_{u}^{1} X_{u}^{2}+f_{x z} X_{u}^{1} X_{u}^{3}+f_{y z} X_{u}^{2} X_{u}^{3}\right]+f_{x x}\left(X_{u}^{1}\right)^{2}+f_{y y}\left(X_{u}^{2}\right)^{2}+f_{z z}\left(X_{u}^{3}\right)^{2} \\
b_{12} & =A\left\|X_{u} \wedge X_{v}\right\| f+f_{x y}\left(X_{u}^{1} X_{v}^{2}+X_{v}^{1} X_{u}^{2}\right)+f_{x z}\left(X_{u}^{1} X_{v}^{3}+X_{v}^{1} X_{u}^{3}\right)+f_{y z}\left(X_{u}^{2} X_{v}^{3}+X_{v}^{2} X_{u}^{3}\right) \\
& +f_{x x} X_{u}^{1} X_{v}^{1}+f_{y y} X_{u}^{2} X_{v}^{2}+f_{z z} X_{u}^{3} X_{v}^{3} \\
b_{22} & =A\left\|X_{u} \wedge X_{v}\right\| g+2\left[f_{x y} X_{v}^{1} X_{v}^{2}+f_{x z} X_{v}^{1} X_{v}^{3}+f_{y z} X_{v}^{2} X_{v}^{3}\right]+f_{x x}\left(X_{v}^{1}\right)^{2}+f_{y y}\left(X_{v}^{2}\right)^{2}+f_{z z}\left(X_{v}^{3}\right)^{2} .
\end{aligned}
$$

Analisando a equação (2.63), podemos demonstrar o seguinte teorema.

Teorema 2.9. Sejam $X$ superfície paramétrica e $f$ superfície implícita que se intersectam tangencialmente no ponto $P=\alpha(t)$. Se $b_{11}^{2}+b_{12}^{2}+b_{22}^{2} \neq 0$, então, o vetor tangente a $\alpha$ no ponto $P$ é dado por

$$
\begin{aligned}
& t=\frac{\omega X_{u}+X_{v}}{\left\|\omega X_{u}+X_{v}\right\|^{\prime}}, \quad \text { se } b_{11} \neq 0 \\
& t=\frac{X_{u}+\mu X_{v}}{\left\|X_{u}+\mu X_{v}\right\|^{\prime}}, \quad \text { se } b_{11}=0 \text { e } b_{22} \neq 0 \\
& t \| X_{u} \text { ou } t \| X_{v}, \quad \text { se } b_{11}=b_{22}=0 \text {, }
\end{aligned}
$$

em que $\omega=\frac{u_{1}^{\prime}}{v_{1}^{\prime}}$ é solução da equação $b_{11} \omega^{2}+2 b_{12} \omega+b_{22}=0$ e $\mu=\frac{v_{1}^{\prime}}{u_{1}^{\prime}}$ é solução da equação $b_{22} \mu^{2}+2 b_{12} \mu+b_{11}=0$, as quais seguem de (2.63). 
Demonstração. Observando a equação (2.63), vemos que, se $b_{11} \neq 0$, não ocorre $v^{\prime}=0$. Neste caso, dividindo (2.63) por $\left(v^{\prime}\right)^{2}$, obtemos

$$
b_{11}(\omega)^{2}+2 b_{12} \omega+b_{22}=0
$$

em que $\omega=\frac{u^{\prime}}{v^{\prime}}$. Logo,

$$
\omega=\frac{-b_{12} \pm \sqrt{b_{12}^{2}-b_{11} b_{22}}}{b_{11}} .
$$

Segue que

$$
t=X_{u} u^{\prime}+X_{v} v^{\prime}=\left(\omega X_{u}+X_{v}\right) v^{\prime}
$$

e, sendo $\|t\|=1$, então $v^{\prime}=\frac{1}{\left\|\omega X_{u}+X_{v}\right\|}$. Portanto, o vetor tangente é dado por

$$
t=\frac{\omega X_{u}+X_{v}}{\left\|\omega X_{u}+X_{v}\right\|} .
$$

Agora, se $b_{11}=0$, com $b_{22} \neq 0$, observando que neste caso $u^{\prime} \neq 0$, definimos $\mu=\frac{v^{\prime}}{u^{\prime}}$, de modo que

$$
b_{22} \mu^{2}+2 b_{12} \mu=0
$$

Segue que $\mu=\frac{-b_{12} \pm b_{12}}{b_{22}} \mathrm{e}$

$$
t=\frac{X_{u}+\mu X_{v}}{\left\|X_{u}+\mu X_{v}\right\|}
$$

Se $b_{11}=b_{22}=0$, então, segue da hipótese $b_{11}^{2}+b_{12}^{2}+b_{22}^{2} \neq 0$ que $b_{12} \neq 0$. Portanto, $u^{\prime}=0$ ou $v^{\prime}=0$, ou seja, como $t=X_{u} u^{\prime}+X_{v} v^{\prime}$, segue que $t$ é paralelo a um dos vetores da base $\left\{X_{u}, X_{v}\right\}$.

A classificação do ponto $P$ de acordo com o sinal do discriminante $\Delta$ segue de maneira análoga ao que fizemos no parágrafo anterior.

\section{Curvatura e Vetor Curvatura}

Analisaremos o caso onde $v^{\prime} \neq 0$. De (2.63), temos que

$$
b_{11}\left(\frac{u^{\prime}}{v^{\prime}}\right)^{2}+2 b_{12}\left(\frac{u^{\prime}}{v^{\prime}}\right)+b_{22}=0
$$




\section{Derivando,}

$$
\frac{1}{\left(v^{\prime}\right)^{3}}\left[b_{11}^{\prime}\left(u^{\prime}\right)^{2} v^{\prime}+2 b_{11} u^{\prime}\left(u^{\prime \prime} v^{\prime}-u^{\prime} v^{\prime \prime}\right)+2\left[b_{12}^{\prime} u^{\prime}\left(v^{\prime}\right)^{2}+b_{12} v^{\prime}\left(u^{\prime \prime} v^{\prime}-u^{\prime} v^{\prime \prime}\right)\right]+b_{22}\left(v^{\prime}\right)^{3}\right]=0 .
$$

Da definição de $\omega$, tem-se $u^{\prime}=\omega v^{\prime}$. Assim, sendo $v^{\prime}=-u^{\prime} \frac{h_{u}}{h_{v}}\left(\right.$ por (2.16)), então $\omega=-\frac{h_{v}}{h_{u}}$. Portanto, por (2.18),

$$
v^{\prime}=\left(E \omega^{2}+2 F \omega+G\right)^{-\frac{1}{2}}
$$

Isto implica que $u^{\prime}=\omega\left(E \omega^{2}+2 F \omega+G\right)^{-\frac{1}{2}}$. Em resumo, temos que

$$
\begin{aligned}
& u^{\prime}=-\omega\left(E \omega^{2}+2 F \omega+G\right)^{-\frac{1}{2}} \\
& v^{\prime}=-\left(E \omega^{2}+2 F \omega+G\right)^{-\frac{1}{2}} .
\end{aligned}
$$

Logo,

$$
\begin{aligned}
0 & =b_{11}^{\prime}\left(\omega v^{\prime}\right)^{2} v^{\prime}+2 b_{11} \omega v^{\prime}\left(u^{\prime \prime} v^{\prime}-\omega v^{\prime} v^{\prime \prime}\right)+2\left[b_{12}^{\prime} \omega\left(v^{\prime}\right)^{3}+b_{12} v^{\prime}\left(u^{\prime \prime} v^{\prime}-\omega v^{\prime} v^{\prime \prime}\right)\right]+b_{22}\left(v^{\prime}\right)^{3} \\
& =2\left[\left(b_{11} \omega+b_{12}\right) v^{\prime} u^{\prime \prime}-\omega\left(b_{11} \omega+b_{12}\right) v^{\prime} v^{\prime \prime}\right]+b_{11}^{\prime} \omega^{2}\left(v^{\prime}\right)^{2}+2 b_{12}^{\prime} \omega\left(v^{\prime}\right)^{2}+b_{22}^{\prime}\left(v^{\prime}\right)^{2}
\end{aligned}
$$

Como $v^{\prime} \neq 0$,

$$
\begin{aligned}
& 0=2\left(b_{11} \omega+b_{12}\right)\left(u^{\prime \prime}-\omega v^{\prime \prime}\right)+v^{\prime}\left(b_{11}^{\prime} \omega^{2}+2 b_{12}^{\prime} \omega+b_{22}^{\prime}\right) \Rightarrow \\
& u^{\prime \prime}-\omega v^{\prime \prime}=-v^{\prime} \frac{b_{11}^{\prime} \omega^{2}+2 b_{12}^{\prime} \omega+b_{22}^{\prime}}{b_{11} \omega+b_{12}}, \quad b_{11} \omega+b_{12} \neq 0 .
\end{aligned}
$$

Portanto,

$$
u^{\prime \prime}-\omega v^{\prime \prime}=a_{1}, \quad b_{11} \omega+b_{12} \neq 0,
$$

onde $a_{1}=-v^{\prime} \frac{b_{11}^{\prime} \omega^{2}+2 b_{12}^{\prime} \omega+b_{22}^{\prime}}{b_{11} \omega+b_{12}}$.

Agora, sendo $\left\langle\alpha^{\prime}, \alpha^{\prime \prime}\right\rangle=0$, segue de (1.4) e da igualdade $u^{\prime}=\omega v^{\prime}$ que

$$
\begin{aligned}
0=\left\langle\alpha^{\prime}, \alpha^{\prime \prime}\right\rangle & =\left\langle X_{u}, X_{u u}\right\rangle\left(u^{\prime}\right)^{3}+2\left\langle X_{u}, X_{u v}\right\rangle\left(u^{\prime}\right)^{2} v^{\prime}+\left\langle X_{u}, X_{v v}\right\rangle u^{\prime}\left(v^{\prime}\right)^{2}+E u^{\prime} u^{\prime \prime}+F u^{\prime} v^{\prime \prime} \\
& +\left\langle X_{v}, X_{u u}\right\rangle\left(u^{\prime}\right)^{2} v^{\prime}+2\left\langle X_{v}, X_{u v}\right\rangle u^{\prime}\left(v^{\prime}\right)^{2}+\left\langle X_{v}, X_{v v}\right\rangle\left(v^{\prime}\right)^{3}+F v^{\prime} u^{\prime \prime}+G v^{\prime} v^{\prime \prime} \\
& =v^{\prime}\left[(E \omega+F) u^{\prime \prime}+(F \omega+G) v^{\prime \prime}+\left\langle X_{u}, X_{u u}\right\rangle \omega^{3}\left(v^{\prime}\right)^{2}+2\left\langle X_{u}, X_{u v}\right\rangle \omega^{2}\left(v^{\prime}\right)^{2}\right. \\
& \left.+\left\langle X_{u}, X_{v v}\right\rangle \omega\left(v^{\prime}\right)^{2}+\left\langle X_{v}, X_{u u}\right\rangle \omega^{2}\left(v^{\prime}\right)^{2}+2\left\langle X_{v}, X_{u v}\right\rangle \omega\left(v^{\prime}\right)^{2}+\left\langle X_{v}, X_{v v}\right\rangle\left(v^{\prime}\right)^{2}\right] .
\end{aligned}
$$

Como $v^{\prime} \neq 0$, então

$$
a_{2} u^{\prime \prime}+a_{3} v^{\prime \prime}=a_{4}
$$


onde

$$
\begin{aligned}
a_{2} & =E \omega+F \\
a_{3} & =F \omega+G \\
a_{4} & =-\left(v^{\prime}\right)^{2}\left[\left\langle X_{u}, X_{u u}\right\rangle \omega^{3}+2\left\langle X_{u}, X_{u v}\right\rangle \omega^{2}+\left\langle X_{u}, X_{v v}\right\rangle \omega+\left\langle X_{v}, X_{u u}\right\rangle \omega^{2}+2\left\langle X_{v}, X_{u v}\right\rangle \omega\right. \\
& \left.+\left\langle X_{v}, X_{v v}\right\rangle\right] .
\end{aligned}
$$

Resolvendo (2.66) e (2.67) para $u^{\prime \prime}$ e $v^{\prime \prime}$ :

$$
\begin{aligned}
& u^{\prime \prime}=\frac{a_{1} a_{3}+a_{4} \omega}{a_{3}+\omega a_{2}} \\
& v^{\prime \prime}=\frac{a_{4}-a_{1} a_{2}}{a_{3}+\omega a_{2}} .
\end{aligned}
$$

Substituindo os valores de $u^{\prime \prime}, u^{\prime}, v^{\prime \prime}, v^{\prime}$ na expressão de $\alpha^{\prime \prime}$ dada no sistema (1.4), obtemos o vetor curvatura $\vec{K}=\alpha^{\prime \prime}$ e a curvatura $\kappa=\sqrt{\langle\vec{K}, \vec{K}\rangle}$. Com isto, temos demonstrado o seguinte resultado.

Teorema 2.10. O vetor curvatura da curva de interseção tangencial $\alpha$ entre uma superfície paramétrica $X$ e uma superfície implícita $f$, no ponto $P=\alpha(t)$,é dado por

$$
\vec{K}=X_{u u}\left(u^{\prime}\right)^{2}+2 X_{u v} u^{\prime} v^{\prime}+X_{v v}\left(v^{\prime}\right)^{2}+X_{u} u^{\prime \prime}+X_{v} v^{\prime \prime},
$$

onde

$$
\begin{array}{ll}
u^{\prime}=\omega\left(E \omega^{2}+2 F \omega+G\right)^{-\frac{1}{2},} & v^{\prime}=\left(E \omega^{2}+2 F \omega+G\right)^{-\frac{1}{2}} \\
u^{\prime \prime}=\frac{a_{1} a_{3}+a_{4} \omega}{a_{3}+\omega a_{2}}, & v^{\prime \prime}=\frac{a_{4}-a_{1} a_{2}}{a_{3}+\omega a_{2}} .
\end{array}
$$

A curvatura de a em P pode ser obtida por $\kappa=\sqrt{\langle\vec{K}, \vec{K}\rangle}$.

\section{Torção}

Se o ponto de interseção tangencial $P$ é de contato isolado ou de ordem $\geq 2$, não há curva de interseção. Se $P$ é um ponto de ramificação, calculamos a torção tomando o limite da torção da curva de interseção transversal neste ponto. Por fim, se temos uma curva de interseção tangencial, podemos calcular $u^{\prime \prime \prime}$ e $v^{\prime \prime \prime}$ diferenciando $u^{\prime \prime}$ e $v^{\prime \prime}$, obtidos anteriormente. Depois, substituindo $u^{\prime}, v^{\prime}, u^{\prime \prime}, v^{\prime \prime}, u^{\prime \prime \prime}$ e $v^{\prime \prime \prime}$ na terceira equação do sistema (1.4), obtemos o vetor $\alpha^{\prime \prime \prime} \mathrm{e}$, então, a torção pode ser calculada por

$$
\tau=\frac{\left\langle\alpha^{\prime \prime \prime}, b\right\rangle}{\kappa} .
$$




\subsubsection{Interseção entre duas Superfícies Implícitas}

Para finalizar o estudo das curvas de interseção no espaço Euclidiano, consideraremos o caso Implícita-Implícita, usando como referência o artigo [1].

Suponha que $f(x, y, z)=0$ e $h(x, y, z)=0$ sejam duas superfícies implícitas regulares que se intersectam tangencialmente em um ponto $P$. Assim, em $P, N^{f} \| N^{h}$, ou seja, $\frac{\nabla f(P)}{\|\nabla f(P)\|}=$ $\pm \frac{\nabla h(P)}{\|\nabla h(P)\|}$, o que pode ser escrito como

$$
\nabla f(P)=A(P) \nabla h(P), \quad A(P)= \pm \frac{\|\nabla f(P)\|}{\|\nabla h(P)\|}
$$

Daí,

$$
\begin{aligned}
& f_{x}(P)=A(P) h_{x}(P) \\
& f_{y}(P)=A(P) h_{y}(P) \\
& f_{z}(P)=A(P) h_{z}(P) .
\end{aligned}
$$

Logo, os pontos de interseção tangencial são determinados resolvendo o seguinte sistema:

$$
f=0, \quad h=0, \quad f_{x}=A h_{x}, f_{y}=A h_{y}, f_{z}=A h_{z} .
$$

\section{Vetor Tangente}

Assuma que as superfícies $f$ e $h$ se intersectam ao longo de uma curva $\beta$ que está parametrizada na variável $x$. Então,

$$
\beta(x)=(x, y(x), z(x)) ; \quad f=0, \quad h=0, \quad f_{x}=A h_{x}, \quad f_{y}=A h_{y}, \quad f_{z}=A h_{z} .
$$

Derivando $\beta(x)=(x, y(x), z(x))$ com respeito a $x$ e usando a equação (2.24), tem-se

$$
\begin{array}{cl}
\dot{\beta}(x)=(1, \dot{y}(x), \dot{z}(x)), \quad & \ddot{\beta}(x)=(0, \ddot{y}(x), \ddot{z}(x)) ; \\
{[\nabla f][\dot{\beta}]=0, \quad[\nabla h][\dot{\beta}]=0 .}
\end{array}
$$

Projetando $\ddot{\beta}$ sobre o normal de ambas as superfícies, temos

$$
\begin{array}{r}
\langle\ddot{\beta}, \nabla f\rangle=A\langle\ddot{\beta}, \nabla h\rangle \Rightarrow \\
{[\nabla f][\ddot{\beta}]=A[\nabla h][\ddot{\beta}] .}
\end{array}
$$

Por (2.39),

$$
\begin{gathered}
-[\dot{\beta}]^{T} H_{1}[\dot{\beta}]=-A[\dot{\beta}]^{T} H_{2}[\dot{\beta}]=-[\dot{\beta}]^{T}\left(A H_{2}\right)[\dot{\beta}] \Rightarrow \\
{[\dot{\beta}]^{T}\left(H_{1}-A H_{2}\right)[\dot{\beta}]=0 .}
\end{gathered}
$$


Explicitamente,

$$
\left[\begin{array}{ccc}
1 & \dot{y} & \dot{z}
\end{array}\right]\left[\begin{array}{ccc}
f_{x x}-A h_{x x} & f_{x y}-A h_{x y} & f_{x z}-A h_{x z} \\
f_{x y}-A h_{x y} & f_{y y}-A h_{y y} & f_{y z}-A h_{y z} \\
f_{x z}-A h_{x z} & f_{y z}-A h_{y z} & f_{z z}-A h_{z z}
\end{array}\right]\left[\begin{array}{c}
1 \\
\dot{y} \\
\dot{z}
\end{array}\right]=0
$$

ou seja,

$$
\begin{aligned}
& \left(f_{y y}-A h_{y y}\right)(\dot{y})^{2}+\left(f_{z z}-A h_{z z}\right)(\dot{z})^{2}+2\left(f_{y z}-A h_{y z}\right) \dot{y} \dot{z}+2\left(f_{x y}-A h_{x y}\right) \dot{y}+2\left(f_{x z}-A h_{x z}\right) \dot{z}+ \\
& f_{x x}-A h_{x x}=0 .
\end{aligned}
$$

A equação (2.68) implica

$$
0=\left[\begin{array}{lll}
f_{x} & f_{y} & f_{z}
\end{array}\right]\left[\begin{array}{c}
1 \\
\dot{y} \\
\dot{z}
\end{array}\right]=\left[f_{x}+\dot{y} f_{y}+\dot{z} f_{z}\right]
$$

Logo,

$$
\begin{aligned}
\dot{y} & =\frac{-f_{x}-\dot{z} f_{z}}{f_{y}}, \quad \text { se } f_{y} \neq 0 \\
\text { ou } & \\
\dot{z} & =\frac{-f_{x}-\dot{y} f_{y}}{f_{z}}, \quad \text { se } f_{z} \neq 0 .
\end{aligned}
$$

Observe que não ocorre $f_{y}=f_{z}=0$. Assuma que $f_{y} \neq 0$. Substituindo na equação (2.69), segue que

$$
b_{11}(\dot{z})^{2}+2 b_{12} \dot{z}+b_{22}=0
$$

onde

$$
\begin{aligned}
& b_{11}=f_{z}^{2}\left(f_{y y}-A h_{y y}\right)+f_{y}^{2}\left(f_{z z}-A h_{z z}\right)-2 f_{y} f_{z}\left(f_{y z}-A h_{y z}\right) \\
& b_{12}=f_{y}^{2}\left(f_{x z}-A h_{x z}\right)+f_{x} f_{z}\left(f_{y y}-A h_{y y}\right)+f_{x} f_{y}\left(f_{y z}-A h_{y z}\right)-f_{y} f_{z}\left(f_{x y}-A h_{x y}\right) \\
& b_{22}=f_{x}^{2}\left(f_{y y}-A h_{y y}\right)+f_{y}^{2}\left(f_{x x}-A h_{x x}\right)-2 f_{x} f_{y}\left(f_{x y}-A h_{x y}\right) .
\end{aligned}
$$

Resolvendo (2.71), temos que

$$
\dot{z}=\frac{-b_{12} \pm \sqrt{b_{12}^{2}-b_{11} b_{22}}}{b_{11}}
$$


o que implica, por (2.70),

$$
\dot{y}=\frac{-f_{x}-f_{z} \frac{-b_{12} \pm \sqrt{b_{12}^{2}-b_{11} b_{22}}}{b_{11}}}{f_{y}} .
$$

Logo,

$$
\begin{aligned}
& \dot{x}=1 \\
& \dot{y}=-\frac{b_{11} f_{x}-b_{12} f_{z} \pm f_{z} \sqrt{b_{12}^{2}-b_{11} b_{22}}}{b_{11} f_{y}} \\
& \dot{z}=\frac{-b_{12} \pm \sqrt{b_{12}^{2}-b_{11} b_{22}}}{b_{11}} .
\end{aligned}
$$

A partir daí, podemos calcular a velocidade, o comprimento de arco e o vetor tangente unitário da curva $\beta$ da seguinte maneira

$$
\begin{aligned}
\|\dot{\beta}(x)\| & =\sqrt{1+\dot{y}^{2}+\dot{z}^{2}} \\
S(x) & =\int_{x_{0}}^{x}\|\dot{\beta}(x)\| d x \\
t(x) & =\frac{\dot{\beta}(x)}{\|\dot{\beta}(x)\|} .
\end{aligned}
$$

A classificação de $P$ de acordo com o discriminante $\Delta$ da equação (2.71) segue assim como fizemos na Subseção 2.2.1.

\section{Curvatura e Vetor Curvatura}

As expressões da curvatura e do vetor curvatura da curva de interseção tangencial entre duas superfícies implícitas são dadas como mostra o teorema a seguir.

Teorema 2.11. O vetor curvatura e a curvatura da curva $\beta(x)=(x, y(x), z(x))$ no ponto de interseção tangencial $P$ entre duas superfícies implícitas $f$ e h são dados, respectivamente, por

$$
\begin{aligned}
\vec{K} & =\frac{\left(-(\dot{y} \ddot{y}+\dot{z} \ddot{z}),\left(1+\dot{z}^{2}\right) \ddot{y}-\dot{y} \dot{z} \ddot{z},\left(1+\dot{y}^{2}\right) \ddot{z}-\dot{y} \dot{z} \ddot{y}\right)}{\left(1+\dot{y}^{2}+\dot{z}^{2}\right)^{2}} \\
\kappa & =\sqrt{\frac{\left(1+\dot{z}^{2}\right) \ddot{y}^{2}-2 \dot{y} \dot{z} \ddot{y} \ddot{z}+\left(1+\dot{y}^{2}\right) \ddot{z}^{2}}{\left(1+\dot{y}^{2}+\dot{z}^{2}\right)^{3}}}
\end{aligned}
$$

em que $\dot{y}, \dot{z}$ e $\ddot{y}, \ddot{z}$ são dados em termos de $f$, h e suas derivadas.

Demonstração. Projetando $\dddot{\beta}$ sobre os vetores normais unitários de ambas as superfícies, obtemos

$$
[\nabla f][\dddot{\beta}]=A[\nabla h][\dddot{\beta}]
$$


Assim, da equação (2.31), segue que

$$
\begin{array}{r}
-3[\dot{\beta}]^{T} H_{1}[\ddot{\beta}]-[\dot{\beta}]^{T} \dot{H}_{1}[\dot{\beta}]=A\left(-3[\dot{\beta}]^{T} H_{2}[\ddot{\beta}]-[\dot{\beta}]^{T} \dot{H}_{2}[\dot{\beta}]\right) \Rightarrow \\
3[\dot{\beta}]^{T}\left(H_{1}-A H_{2}\right)[\ddot{\beta}]=[\dot{\beta}]^{T}\left(A \dot{H}_{2}-\dot{H}_{1}\right)[\dot{\beta}],
\end{array}
$$

o que pode ser escrito como

$$
3\left[\begin{array}{c}
f_{x x}-A h_{x x}+\left(f_{x y}-A h_{x y}\right) \dot{y}+\left(f_{x z}-A h_{x z}\right) \dot{z} \\
f_{x y}-A h_{x y}+\left(f_{y y}-A h_{y y}\right) \dot{y}+\left(f_{y z}-A h_{y z}\right) \dot{z} \\
f_{x z}-A h_{x z}+\left(f_{y z}-A h_{y z}\right) \dot{y}+\left(f_{z z}-A h_{z z}\right) \dot{z}
\end{array}\right]^{T}\left[\begin{array}{c}
0 \\
\ddot{y} \\
\ddot{z}
\end{array}\right]=[\dot{\beta}]^{T}\left(A \dot{H}_{2}-\dot{H}_{1}\right)[\dot{\beta}] .
$$

Além disso, da equação (2.26), tem-se

$$
\left[\begin{array}{lll}
h_{x} & h_{y} & h_{z}
\end{array}\right]\left[\begin{array}{c}
0 \\
\ddot{y} \\
\ddot{z}
\end{array}\right]=-[\dot{\beta}]^{T} H_{2}[\dot{\beta}]
$$

Portanto,

$$
\left[\begin{array}{cc}
3\left(f_{x y}-A h_{x y}+\left(f_{y y}-A h_{y y}\right) \dot{y}+\left(f_{y z}-A h_{y z}\right) \dot{z}\right) & h_{y} \\
3\left(f_{x z}-A h_{x z}+\left(f_{y z}-A h_{y z}\right) \dot{y}+\left(f_{z z}-A h_{z z}\right) \dot{z}\right) & h_{z}
\end{array}\right]^{T}\left[\begin{array}{c}
\ddot{y} \\
\ddot{z}
\end{array}\right]=\left[\begin{array}{c}
{[\dot{\beta}]^{T}\left(A \dot{H}_{2}-\dot{H}_{1}\right)[\dot{\beta}]} \\
-[\dot{\beta}]^{T} H_{2}[\dot{\beta}]
\end{array}\right]
$$

e, então,

$$
\begin{aligned}
{\left[\begin{array}{c}
\ddot{y} \\
\ddot{z}
\end{array}\right]=\frac{1}{\Omega}\left[\begin{array}{cc}
h_{z} & -3\left(f_{x y}-A h_{x y}+\left(f_{y y}-A h_{y y}\right) \dot{y}+\left(f_{y z}-A h_{y z}\right) \dot{z}\right) \\
-h_{y} & 3\left(f_{x z}-A h_{x z}+\left(f_{y z}-A h_{y z}\right) \dot{y}+\left(f_{z z}-A h_{z z}\right) \dot{z}\right)
\end{array}\right]^{T} } \\
\times\left[\begin{array}{c}
{[\dot{\beta}]^{T}\left(A \dot{H}_{2}-\dot{H}_{1}\right)[\dot{\beta}]} \\
-[\dot{\beta}]^{T} H_{2}[\dot{\beta}]
\end{array}\right], \quad \Omega \neq 0,
\end{aligned}
$$

onde $\Omega=\operatorname{det}\left[\begin{array}{cc}3\left(f_{x y}-A h_{x y}+\left(f_{y y}-A h_{y y}\right) \dot{y}+\left(f_{y z}-A h_{y z}\right) \dot{z}\right) & h_{y} \\ 3\left(f_{x z}-A h_{x z}+\left(f_{y z}-A h_{y z}\right) \dot{y}+\left(f_{z z}-A h_{z z}\right) \dot{z}\right) & h_{z}\end{array}\right]^{T}$. Assim, conhecidas as derivadas de primeira e segunda ordem de $y$ e $z$, temos pelas equações (1.2) demonstrado o resultado.

\section{Torção e Derivada de Terceira Ordem}

A seguir, vamos calcular a torção da curva de interseção $\beta$ para o caso Implícita-Implícita. 
Teorema 2.12. A torção $\tau$ no ponto de interseção tangencial $P=\beta(x)$ entre duas superfícies implícitas fe hé dada por

$$
\tau=\frac{\dddot{y} \dddot{z}-\ddot{z} \dddot{y}}{\left(1+\dot{z}^{2}\right) \ddot{y}^{2}-2 \dot{y} \dot{z} \ddot{y} \ddot{z}+\left(1+\dot{y}^{2}\right) \ddot{z}^{2}},
$$

onde as derivadas de y e $z$ são dadas em termos do coeficiente $A= \pm \frac{\|\nabla f\|}{\|\nabla h\|}$ e das derivadas de $f$ e $h$.

Demonstração. Das equações (1.2), temos que

$$
\tau=\frac{\operatorname{det}(\dot{\beta}, \ddot{\beta}, \dddot{\beta})}{\|\dot{\beta} \wedge \ddot{\beta}\|^{2}}=\frac{\ddot{y} \dddot{z}-\ddot{z} \dddot{y}}{\left(1+\dot{z}^{2}\right) \ddot{y}^{2}-2 \dot{y} \dot{z} \ddot{y} \ddot{z}+\left(1+\dot{y}^{2}\right) \dot{z}^{2}} .
$$

Já foram obtidas expressões para $\dot{y}, \dot{z}$ e $\ddot{y}, \ddot{z}$ (ver equações (2.73) e (2.74)). Assim, resta-nos encontrar $\dddot{y}$ e $\dddot{z}$. Projetando $\beta^{(4)}(x)$ sobre os vetores normais unitários de ambas as superfícies, tem-se

$$
[\nabla f]\left[\beta^{(4)}\right]=A[\nabla h]\left[\beta^{(4)}\right]
$$

Logo, da equação (2.33), segue que

$$
\begin{aligned}
& -4[\dot{\beta}]^{T} H_{1}[\dddot{\beta}]-3[\ddot{\beta}]^{T} H_{1}[\ddot{\beta}]-5[\dot{\beta}]^{T} \dot{H}_{1}[\ddot{\beta}]-[\dot{\beta}]^{T} \ddot{H}_{1}[\dot{\beta}] \\
& =A\left(-4[\dot{\beta}]^{T} H_{2}[\ddot{\beta}]-3[\ddot{\beta}]^{T} H_{2}[\ddot{\beta}]-5[\dot{\beta}]^{T} \dot{H}_{2}[\ddot{\beta}]-[\dot{\beta}]^{T} \ddot{H}_{2}[\dot{\beta}]\right) .
\end{aligned}
$$

Daí,

$$
\begin{aligned}
4[\dot{\beta}]^{T}\left(H_{1}-A H_{2}\right)[\dddot{\beta}] & =5[\dot{\beta}]^{T}\left(A \dot{H}_{2}-\dot{H}_{1}\right)[\ddot{\beta}]+3[\ddot{\beta}]^{T}\left(A H_{2}-H_{1}\right)[\ddot{\beta}] \\
& +[\dot{\beta}]^{T}\left(A \ddot{H}_{2}-\ddot{H}_{1}\right)[\dot{\beta}] .
\end{aligned}
$$

Esta última igualdade, junto com a equação (2.31) nos dá

$$
\begin{aligned}
& {\left[\begin{array}{c}
4[\dot{\beta}]^{T}\left(H_{1}-A H_{2}\right) \\
\nabla h
\end{array}\right]\left[\begin{array}{c}
0 \\
\dddot{y} \\
\dddot{z}
\end{array}\right]=\left[\begin{array}{c}
5[\dot{\beta}]^{T}\left(A \dot{H}_{2}-\dot{H}_{1}\right)[\ddot{\beta}]+3[\ddot{\beta}]^{T}\left(A H_{2}-H_{1}\right)[\ddot{\beta}] \\
-3[\dot{\beta}]^{T} H_{2}[\ddot{\beta}]
\end{array}\right]} \\
& +\left[\begin{array}{c}
{[\dot{\beta}]^{T}\left(A \ddot{H}_{2}-\ddot{H}_{1}\right)[\dot{\beta}]} \\
-[\dot{\beta}]^{T} \dot{H}_{2}[\dot{\beta}]
\end{array}\right] .
\end{aligned}
$$

Observando que

$$
4[\dot{\beta}]^{T}\left(H_{1}-A H_{2}\right)=4\left[\begin{array}{c}
f_{x x}-A h_{x x}+\left(f_{x y}-A h_{x y}\right) \dot{y}+\left(f_{x z}-A h_{x z}\right) \dot{z} \\
f_{x y}-A h_{x y}+\left(f_{y y}-A h_{y y}\right) \dot{y}+\left(f_{y z}-A h_{y z}\right) \dot{z} \\
f_{x z}-A h_{x z}+\left(f_{y z}-A h_{y z}\right) \dot{y}+\left(f_{z z}-A h_{z z}\right) \dot{z}
\end{array}\right]^{T},
$$


obtemos

$$
\begin{aligned}
& {\left[\begin{array}{cc}
4\left(f_{x y}-A h_{x y}+\left(f_{y y}-A h_{y y}\right) \dot{y}+\left(f_{y z}-A h_{y z}\right) \dot{z}\right) & h_{y} \\
4\left(f_{x z}-A h_{x z}+\left(f_{y z}-A h_{y z}\right) \dot{y}+\left(f_{z z}-A h_{z z}\right) \dot{z}\right) & h_{z}
\end{array}\right]^{T}\left[\begin{array}{c}
\dddot{y} \\
\dddot{z}
\end{array}\right] } \\
= & {\left[\begin{array}{c}
5[\dot{\beta}]^{T}\left(A \dot{H}_{2}-\dot{H}_{1}\right)[\ddot{\beta}]+3[\ddot{\beta}]^{T}\left(A H_{2}-H_{1}\right)[\ddot{\beta}]+[\dot{\beta}]^{T}\left(A \ddot{H}_{2}-\ddot{H}_{1}\right)[\dot{\beta}] \\
-3[\dot{\beta}]^{T} H_{2}[\ddot{\beta}]-[\dot{\beta}]^{T} \dot{H}_{2}[\dot{\beta}]
\end{array}\right], }
\end{aligned}
$$

ou seja,

$$
\begin{aligned}
{\left[\begin{array}{c}
\dddot{y} \\
\dddot{z}
\end{array}\right] } & =\frac{1}{\rho}\left[\begin{array}{cc}
h_{z} & -4\left(f_{x y}-A h_{x y}+\left(f_{y y}-A h_{y y}\right) \dot{y}+\left(f_{y z}-A h_{y z}\right) \dot{z}\right) \\
-h_{y} & 4\left(f_{x z}-A h_{x z}+\left(f_{y z}-A h_{y z}\right) \dot{y}+\left(f_{z z}-A h_{z z}\right) \dot{z}\right)
\end{array}\right] \\
& \times\left[\begin{array}{c}
5[\dot{\beta}]^{T}\left(A \dot{H}_{2}-\dot{H}_{1}\right)[\ddot{\beta}]+3[\ddot{\beta}]^{T}\left(A H_{2}-H_{1}\right)[\ddot{\beta}]+[\dot{\beta}]^{T}\left(A \ddot{H}_{2}-\ddot{H}_{1}\right)[\dot{\beta}] \\
-3[\dot{\beta}]^{T} H_{2}[\ddot{\beta}]-[\dot{\beta}]^{T} \dot{H}_{2}[\dot{\beta}]
\end{array}\right],
\end{aligned}
$$

onde

$$
\rho=\operatorname{det}\left[\begin{array}{cc}
4\left(f_{x y}-A h_{x y}+\left(f_{y y}-A h_{y y}\right) \dot{y}+\left(f_{y z}-A h_{y z}\right) \dot{z}\right) & h_{y} \\
4\left(f_{x z}-A h_{x z}+\left(f_{y z}-A h_{y z}\right) \dot{y}+\left(f_{z z}-A h_{z z}\right) \dot{z}\right) & h_{z}
\end{array}\right]^{T}
$$

Isto prova o resultado. 


\section{Capítulo 3}

\section{Curvas de Interseção entre duas Superfícies no Espaço de Lorentz-Minkowski}

A partir de agora, consideraremos alguns casos de interseção transversal entre duas superfícies no espaço de Lorentz-Minkowski. O estudo das curvas de interseção neste espaço é relativamente recente, tendo sido iniciado com o tratamento das curvas de interseção transversal entre duas superfícies tipo espaço [3, 2007] e, posteriormente, curvas de interseção transversal não-nulas entre duas superfícies tipo tempo [12, 2014], que serão os casos tratados nas Seções 3.1 e 3.2 deste capítulo. Até então, estes são os únicos casos estudados conhecidos. Sendo assim, existem neste contexto alguns problemas passíveis de estudo pois, como sabemos, cada tipo de interseção (transversal ou tangencial) se divide nos casos Paramétrica-Paramétrica, Paramétrica-Implícita e Implícita-Implícita, os quais se subdividem de acordo com o caráter de causalidade das superfícies envolvidas e também da curva de interseção. Dessa forma, casos como interseção transversal entre superfícies paramétricas tipo luz, ou entre superfícies tipo tempo com curva de interseção tipo luz (e todas as combinações que se pode fazer neste sentido) podem ser considerados para que o estudo das curvas de interseção entre duas superfícies no espaço de Lorentz-Minkowski seja completo.

No que segue, utilizaremos as definições e notações para o espaço de Lorentz-Minkowski $\mathbb{L}^{3}$ introduzidas na Seção 1.2 do capítulo de Preliminares. 


\subsection{Interseção Transversal entre duas Superfícies Paramétricas Tipo Espaço}

Analisaremos neste tópico a curva de interseção entre duas superfícies paramétricas no espaço de Lorentz-Minkowski $\mathbb{L}^{3}$, as quais admitiremos serem tipo espaço e se intersectarem transversalmente. Denotando por $\alpha$ tal curva, encontraremos sua curvatura e sua torção, além de obter condições que dizem quando $\alpha$ é uma geodésica ou uma linha de curvatura das duas superfícies tipo espaço. Em toda esta seção, tomamos como base a referência [3].

Observamos de início que, como os planos tangentes das superfícies consideradas são tipo espaço, então todos os vetores a eles pertencentes são tipo espaço. Em particular, o vetor tangente à curva de interseção $\alpha$, o qual pertence a ambos os planos, é tipo espaço, ou seja, $\alpha$ é uma curva tipo espaço.

Denotando por $X(u, v)$ e $Y(u, v)$ as superfícies paramétricas tipo espaço, cujos vetores normais unitários são $N^{X}$ e $N^{Y}$, tem-se que, como $t$ pertence aos planos tangentes de ambas as superfícies, podemos expressar $t$ por

$$
t=\frac{N^{X} \wedge_{1} N^{Y}}{\left\|N^{X} \wedge_{1} N^{Y}\right\|_{1}}
$$

Observe que $N^{X}$ e $N^{Y}$ são tipo tempo.

Vimos na Seção 1.2 que podemos definir o ângulo hiperbólico entre dois vetores tipo tempo, que é descrito em termos da função cosh. Sendo o vetor curvatura $\vec{K}=\alpha^{\prime \prime}$ normal a $t$, escreveremos

$$
\alpha^{\prime \prime}=\mu N^{X}+\lambda N^{Y}, \quad \mu, \lambda \in \mathbb{R} \text {. }
$$

Projetando $\vec{K}$ sobre os vetores tipo tempo $N^{X}$ e $N^{Y}$ e denotando por $\theta$ o ângulo entre estes, obtemos as respectivas curvaturas normais:

$$
\left\{\begin{array}{l}
\kappa_{n}^{X}=\mu+\lambda \cosh \theta \\
\kappa_{n}^{Y}=\mu \cosh \theta+\lambda
\end{array}, \quad \text { se } N^{X} \text { e } N^{Y}\right. \text { pertencem ao mesmo cone tipo tempo, }
$$

ou

$$
\left\{\begin{array}{l}
\kappa_{n}^{X}=\mu-\lambda \cosh \theta \\
\kappa_{n}^{Y}=-\mu \cosh \theta+\lambda
\end{array}, \quad \text { se } N^{X} \text { e } N^{Y}\right. \text { não pertencem ao mesmo cone tipo tempo. }
$$

Daí, temos o seguinte resultado.

Proposição 3.1. Seja a uma curva de interseção transversal entre as superfícies paramétricas tipo espaço $X$ e Y e $\vec{K}=\alpha^{\prime \prime}$ um vetor não tipo luz. Então a curvatura de $\alpha$ é dada por

$$
\kappa^{2}=\frac{\left|\left(\kappa_{n}^{X}\right)^{2}+\left(\kappa_{n}^{Y}\right)^{2} \pm 2 \kappa_{n}^{X} \kappa_{n}^{Y} \cosh \theta\right|}{\sinh ^{2} \theta} .
$$


Demonstração. Primeiramente, suponha que $N^{X}$ e $N^{Y}$ estão no mesmo cone tipo tempo. Neste caso, $\left\langle N^{X}, N^{Y}\right\rangle_{1}=-\cosh \theta$. Resolvendo o sistema (3.2) para $\mu$ e $\lambda$, obtemos

$$
\begin{aligned}
& \mu=\frac{-\kappa_{n}^{X}+\kappa_{n}^{Y} \cosh \theta}{\sinh ^{2} \theta} \\
& \lambda=\frac{-\kappa_{n}^{Y}+\kappa_{n}^{X} \cosh \theta}{\sinh ^{2} \theta} .
\end{aligned}
$$

Substituindo estes valores em (3.1), segue que

$$
\alpha^{\prime \prime}=\frac{-\kappa_{n}^{X}+\kappa_{n}^{Y} \cosh \theta}{\sinh ^{2} \theta} N^{X}+\frac{-\kappa_{n}^{Y}+\kappa_{n}^{X} \cosh \theta}{\sinh ^{2} \theta} N^{Y} .
$$

Podemos, então, encontrar a curvatura $\kappa=\sqrt{\left|\left\langle\alpha^{\prime \prime}, \alpha^{\prime \prime}\right\rangle_{1}\right|}$ :

$$
\begin{aligned}
\kappa^{2} & =\mid\left(\frac{-\kappa_{n}^{X}+\kappa_{n}^{Y} \cosh \theta}{\sinh ^{2} \theta}\right)^{2}\left\langle N^{X}, N^{X}\right\rangle_{1}+\left(\frac{-\kappa_{n}^{Y}+\kappa_{n}^{X} \cosh \theta}{\sinh ^{2} \theta}\right)^{2}\left\langle N^{Y}, N^{Y}\right\rangle_{1} \\
& +2\left(\frac{-\kappa_{n}^{X}+\kappa_{n}^{Y} \cosh \theta}{\sinh ^{2} \theta}\right)\left(\frac{-\kappa_{n}^{Y}+\kappa_{n}^{X} \cosh \theta}{\sinh ^{2} \theta}\right)\left\langle N^{X}, N^{Y}\right\rangle_{1} \mid \\
& =\mid\left(\frac{-\kappa_{n}^{X}+\kappa_{n}^{Y} \cosh \theta}{\sinh ^{2} \theta}\right)^{2}+\left(\frac{-\kappa_{n}^{Y}+\kappa_{n}^{X} \cosh \theta}{\sinh ^{2} \theta}\right)^{2} \\
& +2\left(\frac{-\kappa_{n}^{X}+\kappa_{n}^{Y} \cosh \theta}{\sinh ^{2} \theta}\right)\left(\frac{-\kappa_{n}^{Y}+\kappa_{n}^{X} \cosh \theta}{\sinh ^{2} \theta}\right) \cosh \theta \mid \\
& =\frac{\left|\left(\kappa_{n}^{X}\right)^{2}+\left(\kappa_{n}^{Y}\right)^{2}-2 \kappa_{n}^{X} \kappa_{n}^{Y} \cosh \theta\right|}{\sinh ^{2} \theta} .
\end{aligned}
$$

Analogamente, se $N^{X}$ e $N^{Y}$ não estão no mesmo cone tipo tempo, então $\left\langle N^{X}, N^{Y}\right\rangle_{1}=\cosh \theta$. Logo,

$$
\begin{aligned}
& \mu=-\frac{\kappa_{n}^{X}+\kappa_{n}^{Y} \cosh \theta}{\sinh ^{2} \theta} \\
& \lambda=-\frac{\kappa_{n}^{Y}+\kappa_{n}^{X} \cosh \theta}{\sinh ^{2} \theta}
\end{aligned}
$$

o que implica

$$
\kappa^{2}=\frac{\left|\left(\kappa_{n}^{X}\right)^{2}+\left(\kappa_{n}^{Y}\right)^{2}+2 \kappa_{n}^{X} \kappa_{n}^{Y} \cosh \theta\right|}{\sinh ^{2} \theta} .
$$

Vamos agora calcular a torção da curva de interseção $\alpha$. Inicialmente, observamos que, sendo $\alpha$ tipo espaço então, se $\alpha^{\prime \prime}$ é tipo espaço ou tipo tempo, tem-se $\alpha^{\prime \prime}=\kappa n$ e, se $\alpha^{\prime \prime}$ é tipo luz, então $\alpha^{\prime \prime}=n$ (ver equações (1.24), (1.25) e (1.26)). Derivando-se estas expressões, obtemos

1. Se $\alpha^{\prime \prime}$ é tipo espaço: $\alpha^{\prime \prime \prime}=-\kappa^{2} t+\kappa^{\prime} n+\kappa \tau b$.

2. Se $\alpha^{\prime \prime}$ é tipo tempo:

$\alpha^{\prime \prime \prime}=\kappa^{2} t+\kappa^{\prime} n+\kappa \tau b$.

3. Se $\alpha^{\prime \prime}$ é tipo luz:

$\alpha^{\prime \prime \prime}=\tau n$. 
A torção pode ser obtida destas equações por

$$
\begin{array}{ll}
\text { 1. Se } \alpha^{\prime \prime} \text { é tipo espaço: } & \tau=-\frac{\left\langle\alpha^{\prime \prime \prime}, b\right\rangle_{1}}{\kappa} . \\
\text { 2. Se } \alpha^{\prime \prime} \text { é tipo tempo: } & \tau=\frac{\left\langle\alpha^{\prime \prime \prime}, b\right\rangle_{1}}{\kappa} . \\
\text { 3. Se } \alpha^{\prime \prime} \text { é tipo luz: } & \tau=\left\langle\alpha^{\prime \prime \prime}, b\right\rangle_{1} .
\end{array}
$$

Além disso, como $\operatorname{Span}\left\{N^{X}, N^{Y}\right\}=\operatorname{Span}\{n, b\}$, podemos escrever os termos $\kappa^{\prime} n+\kappa \tau b$ e $\tau n$ que aparecem nas expressões do vetor $\alpha^{\prime \prime \prime} \operatorname{como} \gamma N^{X}+\delta N^{Y}$, com $\gamma, \delta \in \mathbb{R}$, obtendo-se

$$
\begin{array}{ll}
\text { 1. Se } \alpha^{\prime \prime} \text { é tipo espaço: } & \alpha^{\prime \prime \prime}=-\kappa^{2} t+\gamma N^{X}+\delta N^{Y} . \\
\text { 2. Se } \alpha^{\prime \prime} \text { é tipo tempo: } & \alpha^{\prime \prime \prime}=\kappa^{2} t+\gamma N^{X}+\delta N^{Y} . \\
\text { 3. Se } \alpha^{\prime \prime} \text { é tipo luz: } & \alpha^{\prime \prime \prime}=\gamma N^{X}+\delta N^{Y} .
\end{array}
$$

Denotando por $\lambda_{n}$ a projeção de $\alpha^{\prime \prime \prime}$ sobre o normal da superfície, segue que

$$
\left\{\begin{array}{l}
\gamma=\frac{\lambda_{n}^{X}-\lambda_{n}^{Y} \cosh \theta}{\sinh ^{2} \theta} \\
\delta=\frac{\lambda_{n}^{Y}-\lambda_{n}^{X} \cosh \theta}{\sinh ^{2} \theta}
\end{array}, \quad \text { se } N^{X} \text { e } N^{Y}\right. \text { estão no mesmo cone tipo tempo, }
$$

ou

$$
\left\{\begin{array}{l}
\gamma=\frac{\lambda_{n}^{X}+\lambda_{n}^{Y} \cosh \theta}{\sinh ^{2} \theta} \\
\delta=\frac{\lambda_{n}^{Y}+\lambda_{n}^{X} \cosh \theta}{\sinh ^{2} \theta}
\end{array}, \quad \text { se } N^{X} \text { e } N^{Y}\right. \text { não estão no mesmo cone tipo tempo. }
$$

Dessa forma, podemos escrever a torção em termos dos escalares $\lambda_{n}^{X}, \lambda_{n}^{Y}$ e dos normais $N^{X}$, $N^{Y}$, como mostra a seguinte proposição.

Proposição 3.2. Suponha que a seja uma curva de interseção entre as superfícies tipo espaço X e $Y$. Então, sua torção $\tau$ é dada da seguinte forma:
1. Se $\alpha^{\prime \prime}$ é tipo espaço: $\quad \tau=\frac{-1}{\kappa \sinh ^{2} \theta}\left[\left(\lambda_{n}^{X} \pm \lambda_{n}^{Y} \cosh \theta\right)\left\langle N^{X}, b\right\rangle_{1}+\left(\lambda_{n}^{Y} \pm \lambda_{n}^{X} \cosh \theta\right)\left\langle N^{Y}, b\right\rangle_{1}\right]$.
2. Se $\alpha^{\prime \prime}$ é tipo tempo: $\quad \tau=\frac{1}{\kappa \sinh ^{2} \theta}\left[\left(\lambda_{n}^{X} \pm \lambda_{n}^{Y} \cosh \theta\right)\left\langle N^{X}, b\right\rangle_{1}+\left(\lambda_{n}^{Y} \pm \lambda_{n}^{X} \cosh \theta\right)\left\langle N^{Y}, b\right\rangle_{1}\right]$.
3. Se $\alpha^{\prime \prime}$ é tipo luz: $\quad \tau=\frac{1}{\sinh ^{2} \theta}\left[\left(\lambda_{n}^{X} \pm \lambda_{n}^{Y} \cosh \theta\right)\left\langle N^{X}, b\right\rangle_{1}+\left(\lambda_{n}^{Y} \pm \lambda_{n}^{X} \cosh \theta\right)\left\langle N^{Y}, b\right\rangle_{1}\right]$. 
Demonstração. Respeitando o fato de $N^{X}$ e $N^{Y}$ estarem no mesmo cone tipo tempo ou não, temos das equações (3.7) e (3.8) que

$$
\left\{\begin{array}{l}
\gamma=\frac{\lambda_{n}^{X} \pm \lambda_{n}^{Y} \cosh \theta}{\sinh ^{2} \theta} \\
\delta=\frac{\lambda_{n}^{Y} \pm \lambda_{n}^{X} \cosh \theta}{\sinh ^{2} \theta}
\end{array}\right.
$$

Substituindo estes valores nas equações em (3.6), segue que

1. Se $\alpha^{\prime \prime}$ é tipo espaço: $\quad \alpha^{\prime \prime \prime}=-\kappa^{2} t+\frac{\lambda_{n}^{X} \pm \lambda_{n}^{Y} \cosh \theta}{\sinh ^{2} \theta} N^{X}+\frac{\lambda_{n}^{Y} \pm \lambda_{n}^{X} \cosh \theta}{\sinh ^{2} \theta} N^{Y}$,

2. Se $\alpha^{\prime \prime}$ é tipo tempo: $\quad \alpha^{\prime \prime \prime}=\kappa^{2} t+\frac{\lambda_{n}^{X} \pm \lambda_{n}^{Y} \cosh \theta}{\sinh ^{2} \theta} N^{X}+\frac{\lambda_{n}^{Y} \pm \lambda_{n}^{X} \cosh \theta}{\sinh ^{2} \theta} N^{Y}$,

3. Se $\alpha^{\prime \prime}$ é tipo luz: $\quad \alpha^{\prime \prime \prime}=\frac{\lambda_{n}^{X} \pm \lambda_{n}^{Y} \cosh \theta}{\sinh ^{2} \theta} N^{X}+\frac{\lambda_{n}^{Y} \pm \lambda_{n}^{X} \cosh \theta}{\sinh ^{2} \theta} N^{Y}$, onde $\lambda_{n}^{X}$ e $\lambda_{n}^{Y}$ são obtidos pela equação (2.10). Aplicando o resultado obtido para $\alpha^{\prime \prime \prime}$ nas respectivas expressões da torção $\tau$, dadas por (3.5), a proposição segue.

Os resultados a seguir caracterizam a curva de interseção $\alpha$. Considere o triedro de Darboux para as superfícies $X$ e $Y,\left\{t, V^{X}, N^{X}\right\}$ e $\left\{t, V^{Y}, N^{Y}\right\}$, onde $V^{X}=N^{X} \wedge_{1} t$ e $V^{Y}=N^{Y} \wedge_{1} t$. $O$ seguinte lema nos dá uma expressão para a curvatura geodésica de $X$ e $Y$ em termos das respectivas curvaturas normais.

Lema 3.1. Seja a a curva (tipo espaço) de interseção transversal entre as superfícies paramétricas tipo espaço X e Y. Então

$$
\begin{aligned}
\kappa_{g}^{X} & =\frac{-\kappa_{n}^{X} \cosh \theta \pm \kappa_{n}^{Y}}{\sinh \theta} \\
\kappa_{g}^{Y} & =\frac{\kappa_{n}^{Y} \cosh \theta \pm \kappa_{n}^{X}}{\sinh \theta} .
\end{aligned}
$$

Demonstração. Note que

$$
V^{X}=N^{X} \wedge_{1} t=\frac{N^{X} \wedge_{1}\left(N^{X} \wedge_{1} N^{Y}\right)}{\left\|N^{X} \wedge_{1} N^{Y}\right\|_{1}}=\frac{\left\langle N^{X}, N^{X}\right\rangle_{1} N^{Y}-\left\langle N^{X}, N^{Y}\right\rangle_{1} N^{X}}{\left\|N^{X} \wedge_{1} N^{Y}\right\|_{1}}
$$

onde $\left\|N^{X} \wedge_{1} N^{Y}\right\|_{1}^{2}=\left|\left\langle N^{X} \wedge_{1} N^{Y}, N^{X} \wedge_{1} N^{Y}\right\rangle_{1}\right|=\left|\operatorname{det}\left[\begin{array}{cc}\left\langle N^{X}, N^{Y}\right\rangle_{1} & \left\langle N^{Y}, N^{Y}\right\rangle_{1} \\ \left\langle N^{X}, N^{X}\right\rangle_{1} & \left\langle N^{X}, N^{Y}\right\rangle_{1}\end{array}\right]\right|=\sinh ^{2} \theta$ Logo,

$$
V^{X}= \begin{cases}\frac{\cosh \theta N^{X}-N^{Y}}{\sinh \theta}, & \text { se } N^{X} \text { e } N^{Y} \text { estão no mesmo cone tipo tempo, } \\ \frac{-\cosh \theta N^{X}-N^{Y}}{\sinh \theta}, & \text { se } N^{X} \text { e } N^{Y} \text { não estão no mesmo cone tipo tempo. }\end{cases}
$$


Analogamente, tem-se

$$
V^{Y}= \begin{cases}\frac{N^{X}-\cosh \theta N^{Y}}{\sinh \theta}, & \text { se } N^{X} \text { e } N^{Y} \text { estão no mesmo cone tipo tempo, } \\ \frac{N^{X}+\cosh \theta N^{Y}}{\sinh \theta}, & \text { se } N^{X} \text { e } N^{Y} \text { não estão no mesmo cone tipo tempo. }\end{cases}
$$

O vetor curvatura $\alpha^{\prime \prime}$, sendo perpendicular a $t$, deve estar no plano gerado por $N^{X}$ e $N^{Y}$. Logo, existem $\lambda, \mu \in \mathbb{R}$ tais que

$$
\alpha^{\prime \prime}=\lambda N^{X}+\mu N^{Y}
$$

o que nos dá uma expressão para $\kappa_{n}^{X}$ e $\kappa_{n}^{Y}$ em termos de $\lambda$ e $\mu$. Resolvendo o sistema obtido para $\lambda$ e $\mu$, encontramos

$$
\begin{aligned}
& \lambda= \begin{cases}\frac{-\kappa_{n}^{X}+\kappa_{n}^{Y} \cosh \theta}{\sinh ^{2} \theta}, & \text { se } N^{X} \text { e } N^{Y} \text { estão no mesmo cone tipo tempo, } \\
\frac{\kappa_{n}^{X}+\kappa_{n}^{Y} \cosh \theta}{\sinh ^{2} \theta}, & \text { se } N^{X} \text { e } N^{Y} \text { não estão no mesmo cone tipo tempo. }\end{cases} \\
& \mu= \begin{cases}\frac{-\kappa_{n}^{Y}+\kappa_{n}^{X} \cosh \theta}{\sinh ^{2} \theta}, & \text { se } N^{X} \text { e } N^{Y} \text { estão no mesmo cone tipo tempo, } \\
\frac{\kappa_{n}^{Y}+\kappa_{n}^{X} \cosh \theta}{\sinh ^{2} \theta}, & \text { se } N^{X} \text { e } N^{Y} \text { não estão no mesmo cone tipo tempo. }\end{cases}
\end{aligned}
$$

Portanto, por (3.12) e (3.14),

$$
\begin{aligned}
\kappa_{g}^{X} & =\left\langle\alpha^{\prime \prime}, V^{X}\right\rangle_{1}=\frac{1}{\sinh \theta}\left\langle\lambda N^{X}+\mu N^{Y}, \pm \cosh \theta N^{X}-N^{Y}\right\rangle_{1}=\frac{1}{\sinh \theta}\left(-\mu \sinh ^{2} \theta\right) \\
& =-\mu \sinh \theta=\frac{-\kappa_{n}^{X} \cosh \theta \pm \kappa_{n}^{Y}}{\sinh \theta} .
\end{aligned}
$$

De maneira análoga mostra-se que $\kappa_{g}^{Y}=\lambda \sinh \theta=\frac{\kappa_{n}^{Y} \cosh \theta \pm \kappa_{n}^{X}}{\sinh \theta}$.

O próximo lema nos dá uma expressão para a derivada do ângulo hiperbólico $\theta$ ao longo da curva de interseção $\alpha$, formado pelos vetores normais às superfícies $X$ e $Y$, em termos das torções geodésicas $\kappa_{g}^{X}$ e $\kappa_{g}^{Y}$, o que será de muita importância para a demonstração dos próximos resultados.

Lema 3.2. Suponha que as superfícies tipo espaço $X$ e $Y$ se intersectam transversalmente ao longo de uma curva a e seja $\theta$ o ângulo hiperbólico entre $N^{X}$ e $N^{Y}$ num ponto de $\alpha$. Então

$$
\begin{cases}\frac{d \theta}{d s}=\tau_{g}^{X}-\tau_{g}^{Y}, & \text { se } N^{X} \text { e } N^{Y} \text { estão no mesmo cone tipo tempo, } \\ \frac{d \theta}{d s}=-\tau_{g}^{X}+\tau_{g}^{Y}, & \text { se } N^{X} \text { e } N^{Y} \text { não estão no mesmo cone tipo tempo. }\end{cases}
$$


Demonstração. Sabemos que (ver equações (1.28))

$$
\begin{aligned}
& \left(N^{X}\right)^{\prime}=\kappa_{n}^{X} t+\tau_{g}^{X} V^{X} \\
& \left(N^{Y}\right)^{\prime}=\kappa_{n}^{Y} t+\tau_{g}^{Y} V^{Y} .
\end{aligned}
$$

Suponha inicialmente que $N^{X}$ e $N^{Y}$ estão no mesmo cone tipo tempo. Neste caso, $-\cosh \theta=$ $\left\langle N^{X}, N^{Y}\right\rangle_{1}$. Derivando esta expressão com respeito a $s$ e aplicando as equações (3.12), (3.13) e (3.15), tem-se

$$
\begin{aligned}
-\sinh \theta \frac{d \theta}{d s} & =\left\langle\left(N^{X}\right)^{\prime}, N^{Y}\right\rangle_{1}+\left\langle N^{X},\left(N^{Y}\right)^{\prime}\right\rangle_{1} \\
& =\left\langle\kappa_{n}^{X} t+\tau_{g}^{X} V^{X}, N^{Y}\right\rangle_{1}+\left\langle N^{X}, \kappa_{n}^{Y} t+\tau_{g}^{Y} V^{Y}\right\rangle_{1} \\
& =\tau_{g}^{X}\left\langle V^{X}, N^{Y}\right\rangle_{1}+\tau_{g}^{Y}\left\langle N^{X}, V^{Y}\right\rangle_{1} \\
& =\tau_{g}^{X}\left\langle\frac{\cosh \theta N^{X}-N^{Y}}{\sinh \theta}, N^{Y}\right\rangle_{1}+\tau_{g}^{Y}\left\langle N^{X}, \frac{N^{X}-\cosh \theta N^{Y}}{\sinh \theta}\right\rangle_{1} \\
& =\tau_{g}^{X} \frac{\cosh { }^{2} \theta+1}{\sinh \theta}+\tau_{g}^{Y} \frac{-1+\cosh ^{2} \theta}{\sinh \theta} \\
& =-\tau_{g}^{X} \sinh \theta+\tau_{g}^{Y} \sinh \theta,
\end{aligned}
$$

de onde se tem

$$
\frac{d \theta}{d s}=\tau_{g}^{X}-\tau_{g}^{Y}
$$

A outra igualdade segue de maneira inteiramente análoga.

Estabelecidos estes resultados, mostraremos que, sob as hipóteses triviais consideradas nesta seção, dizer que a curva de interseção $\alpha$ entre duas superfícies é geodésica de ambas as superfícies equivale a dizer que ela é curva assintótica delas.

Teorema 3.1. Suponha que as superfícies tipo espaço $X$ e $Y$ se intersectam transversalmente ao longo de $\alpha$, que é uma curva tipo espaço. Então, a é uma geodésica de X e Y se, e somente se, $\alpha$ é uma curva assintótica de X e Y.

Demonstração. Suponha que $\alpha$ seja uma geodésica de $X$ e $Y$. Isto significa que $\kappa_{g}^{X}=\kappa_{g}^{Y}=0$. Logo, pelas equações (3.10),

$$
\left\{\begin{aligned}
-\kappa_{n}^{X} \cosh \theta \pm \kappa_{n}^{Y} & =0 \\
\kappa_{n}^{Y} \cosh \theta \pm \kappa_{n}^{X} & =0 .
\end{aligned}\right.
$$

Resolvendo para $\kappa_{n}^{X}$ e $\kappa_{n}^{Y}$, tem-se $\kappa_{n}^{X}=\kappa_{n}^{Y}=0$, ou seja, $\alpha$ é uma curva assintótica de $X$ e $Y$.

Reciprocamente, se $\alpha$ é assintótica, segue diretamente das equações (3.10) que $\alpha$ é uma geodésica. 
Para finalizar esta seção, mostraremos que, sob determinadas hipóteses, podemos decidir se $\alpha$ é linha de curvatura das duas superfícies apenas conhecendo o ângulo formado pelos seus normais.

Teorema 3.2 (Análogo ao Teorema de Joachimstahl). Suponha que as superfícies tipo espaço X $e$ $Y$ se intersectam transversalmente ao longo da curva $\alpha$ e seja $\theta$ o ângulo hiperbólico formado por $N^{X} e$ $N^{Y}$. Se a é uma linha de curvatura de X, então a é uma linha de curvatura de $Y$ se, e somente se, $\theta$ é constante.

Demonstração. A curva $\alpha$ ser linha de curvatura de $X$ significa que $\tau_{g}^{X}=0$. Se $\alpha$ é uma linha de curvatura de $Y$, tem-se ainda que $\tau_{g}^{Y}=0$. Logo, pelo Lema $3.2, \frac{d \theta}{d s}=0$, ou seja, $\theta$ é constante.

Reciprocamente, se $\theta$ é constante, então $\frac{d \theta}{d s}=0$, o que implica, também pelo Lema 3.2, que $\tau_{g}^{Y}=\tau_{g}^{X}=0$. Logo, $\alpha$ é linha de curvatura de $Y$.

\subsection{Interseção Transversal Não-Nula entre duas Superfícies Pa- ramétricas Tipo Tempo}

Nesta seção, caracterizaremos a curva de interseção transversal entre duas superfícies tipo tempo, considerando tal curva não-nula (ou não tipo luz), e obteremos, como parte desta análise, sua curvatura e sua torção. Utilizamos como base a referência citada em [12]. Lembramos que dizer que uma superfície é tipo tempo significa que seu plano tangente é um subespaço de $\mathbb{L}^{3}$ tipo tempo.

Sejam $X=X(u, v)$ e $Y=Y(u, v)$ superfícies paramétricas tipo tempo e $\alpha=\alpha(s)$ a curva de interseção transversal entre $X$ e $Y$. Note que o vetor tangente a $\alpha$ está tanto sobre o plano tangente a $X$ quanto sobre o plano tangente a $Y$, os quais são tipo tempo. Logo, $t$ é perpendicular a $N^{X}$ e a $N^{Y}$, o que implica que $t$ é paralelo a $N^{X} \wedge_{1} N^{Y}$. Daí, sendo $N^{X} \wedge_{1} N^{Y} \neq 0$, o vetor tangente pode ser expresso por

$$
t=\frac{N^{X} \wedge_{1} N^{Y}}{\left\|N^{X} \wedge_{1} N^{Y}\right\|_{1}}
$$

onde $N^{X}$ e $N^{Y}$ são os vetores normais a $X$ e a $Y$, respectivamente.

Observe que $N^{X}$ e $N^{Y}$ são vetores tipo espaço pois $N^{X} \in\left(T_{p} X\right)^{\perp}$ e $N^{Y} \in\left(T_{p} Y\right)^{\perp}$ (ver Proposição 1.7).

O fato de o vetor $t$ estar em um espaço tipo tempo permite que ele seja tipo espaço, tipo luz ou tipo tempo. O próximo resultado nos diz quando cada caso ocorre. Vamos denotar 
$\left\langle N^{X}, N^{Y}\right\rangle_{1}=\rho$. Pela propriedade dada no item 4 da Proposição 1.12, tem-se que

$$
\left\langle N^{X} \wedge_{1} N^{Y}, N^{X} \wedge_{1} N^{Y}\right\rangle_{1}=\left|\begin{array}{cc}
\left\langle N^{X}, N^{Y}\right\rangle_{1} & \left\langle N^{Y}, N^{Y}\right\rangle_{1} \\
\left\langle N^{X}, N^{X}\right\rangle_{1} & \left\langle N^{X}, N^{Y}\right\rangle_{1}
\end{array}\right|=\rho^{2}-1
$$

Desta forma, o número $\rho^{2}-1$ caracteriza o vetor $t$, pois

$$
\langle t, t\rangle_{1}=\frac{\rho^{2}-1}{\left\|N^{X} \wedge_{1} N^{Y}\right\|_{1}^{2}} .
$$

Temos, assim, a seguinte proposição.

Proposição 3.3. Sejam $X(u, v)$ e $Y(u, v)$ superfícies paramétricas tipo tempo em $\mathbb{L}^{3}$ e $\alpha$ a curva de interseção transversal entre $X$ e $Y$.

1. $\alpha$ é tipo tempo se, e somente se, $\rho \in(-1,1)$.

2. $\alpha$ é tipo luz se, e somente se, $\rho= \pm 1$.

3. $\alpha$ é tipo espaço se, e somente se, $\rho \in \mathbb{R} \backslash[-1,1]$.

A partir de agora, consideraremos $\alpha$ não nula (ou não tipo luz). Uma vez que $N^{X}$ e $N^{Y}$ são ortogonais a $t$, o vetor curvatura de $\alpha$, sendo perpendicular a $t$, deve estar no plano gerado por $N^{X}$ e $N^{Y}$ (que são L.I., pois a interseção é transversal). Logo, existem $\lambda, \mu \in \mathbb{R}$ tais que

$$
\alpha^{\prime \prime}=\lambda N^{X}+\mu N^{Y}
$$

Sendo $\kappa_{n}^{X}$ e $\kappa_{n}^{Y}$ as curvaturas normais de $X$ e $Y$ (ver Definições 1.35 e 1.36), tem-se

$$
\begin{aligned}
& \pm \kappa_{n}^{X}=\lambda+\mu \rho \\
& \pm \kappa_{n}^{Y}=\lambda \rho+\mu,
\end{aligned}
$$

onde o sinal de $\kappa_{n}$ depende de $\alpha$ ser tipo tempo ou tipo espaço. Resolvendo este sistema para $\lambda$ e $\mu, \operatorname{com} \alpha$ não nula (isto é, $1-\rho^{2} \neq 0$ ), obtemos

$$
\lambda= \pm \frac{\kappa_{n}^{X}-\rho \kappa_{n}^{Y}}{1-\rho^{2}}, \quad \mu= \pm \frac{\kappa_{n}^{Y}-\rho \kappa_{n}^{X}}{1-\rho^{2}} .
$$

Substituindo em (3.18):

$$
\alpha^{\prime \prime}= \pm\left(\frac{\kappa_{n}^{X}-\rho \kappa_{n}^{Y}}{1-\rho^{2}} N^{X}+\frac{\kappa_{n}^{Y}-\rho \kappa_{n}^{X}}{1-\rho^{2}} N^{Y}\right)
$$

Temos, portanto, o seguinte resultado. 
Proposição 3.4. Seja a a curva de interseção transversal, não nula, entre as superfícies paramétricas tipo tempo X e Y e considere o vetor curvatura $\alpha^{\prime \prime}$ tipo tempo ou tipo espaço. Então, a curvatura $\kappa$ é dada por

$$
\kappa^{2}=\frac{\left|\left(\kappa_{n}^{X}\right)^{2}+\left(\kappa_{n}^{Y}\right)^{2}-2 \rho \kappa_{n}^{X} \kappa_{n}^{Y}\right|}{1-\rho^{2}} .
$$

Demonstração. Segue da definição de $\kappa$ e da equação (3.20) que

$$
\begin{aligned}
\kappa^{2}=\left|\left\langle\alpha^{\prime \prime}, \alpha^{\prime \prime}\right\rangle_{1}\right| & =\left|\left(\frac{\kappa_{n}^{X}-\rho \kappa_{n}^{Y}}{1-\rho^{2}}\right)^{2}+2 \rho\left(\frac{\kappa_{n}^{X}-\rho \kappa_{n}^{Y}}{1-\rho^{2}}\right)\left(\frac{\kappa_{n}^{Y}-\rho \kappa_{n}^{X}}{1-\rho^{2}}\right)+\left(\frac{\kappa_{n}^{Y}-\rho \kappa_{n}^{X}}{1-\rho^{2}}\right)^{2}\right| \\
& =\frac{1}{1-\rho^{2}}\left|\left(\kappa_{n}^{X}\right)^{2}+\left(\kappa_{n}^{Y}\right)^{2}-2 \rho \kappa_{n}^{X} \kappa_{n}^{Y}\right| .
\end{aligned}
$$

A hipótese de $t^{\prime}$ ser tipo tempo ou espaço na proposição anterior se deve ao fato de não definirmos curvatura quando $t^{\prime}$ é tipo luz. Lembremos que, se $\alpha$ é tipo tempo, então $\alpha^{\prime \prime}=\kappa n$ (ver equações (1.23)). Derivando isto e substituindo no resultado a expressão $n^{\prime}=\kappa t+\tau b$, dada pelas equações de Frenet, tem-se

$$
\alpha^{\prime \prime \prime}=\kappa^{2} t+\kappa^{\prime} n+\kappa \tau b
$$

E, assim como comentado na seção anterior, se $\alpha$ é tipo espaço, temos pelas equações (1.24), (1.25) e (1.26) os seguintes casos:

$$
\begin{array}{ll}
\text { 1. Se } \alpha^{\prime \prime} \text { é tipo espaço: } & \alpha^{\prime \prime \prime}=-\kappa^{2} t+\kappa^{\prime} n+\kappa \tau b . \\
\text { 2. Se } \alpha^{\prime \prime} \text { é tipo tempo: } & \alpha^{\prime \prime \prime}=\kappa^{2} t+\kappa^{\prime} n+\kappa \tau b . \\
\text { 3. Se } \alpha^{\prime \prime} \text { é tipo luz: } & \alpha^{\prime \prime \prime}=\tau n .
\end{array}
$$

A torção pode ser obtida destas equações por

1. Se $\alpha$ é tipo tempo:

$$
\tau=\frac{\left\langle\alpha^{\prime \prime \prime}, b\right\rangle_{1}}{\kappa}
$$

2. Se $\alpha$ e $\alpha^{\prime \prime}$ são tipo espaço:

$$
\tau=-\frac{\left\langle\alpha^{\prime \prime \prime}, b\right\rangle_{1}}{\kappa}
$$

3. Se $\alpha$ é tipo espaço e $\alpha^{\prime \prime}$ é tipo tempo: $\quad \tau=\frac{\left\langle\alpha^{\prime \prime \prime}, b\right\rangle_{1}}{\kappa}$.

4. Se $\alpha$ é tipo espaço e $\alpha^{\prime \prime}$ é tipo luz:

$$
\tau=\left\langle\alpha^{\prime \prime \prime}, b\right\rangle_{1}
$$


Como os normais $N^{X}$ e $N^{Y}$ estão no plano normal (o plano gerado por $n$ e $b$ ), então os termos $\kappa^{\prime} n+\kappa \tau b$ e $\tau n$ nas equações (3.22) e (3.23), que também são vetores no plano normal, podem ser escritos como combinação linear de $N^{X}$ e $N^{Y}$. Assim, se $\alpha$ é tipo tempo,

$$
\alpha^{\prime \prime \prime}=\kappa^{2} t+\gamma N^{X}+\delta N^{Y}
$$

e, se $\alpha$ é tipo espaço,

$$
\begin{array}{ll}
\text { 1. Se } \alpha^{\prime \prime} \text { é tipo espaço: } & \alpha^{\prime \prime \prime}=-\kappa^{2} t+\gamma N^{X}+\delta N^{Y}, \\
\text { 2. Se } \alpha^{\prime \prime} \text { é tipo tempo: } & \alpha^{\prime \prime \prime}=\kappa^{2} t+\gamma N^{X}+\delta N^{Y}, \\
\text { 3. Se } \alpha^{\prime \prime} \text { é tipo luz: } & \alpha^{\prime \prime \prime}=\gamma N^{X}+\delta N^{Y},
\end{array}
$$

onde $\gamma, \delta \in \mathbb{R}$.

Em todo caso, ao projetarmos $\alpha^{\prime \prime \prime}$ sobre o normal da superfície em $\alpha(s)$, denotando isto por $\lambda_{n}$, obtemos

$$
\begin{aligned}
& \lambda_{n}^{X}=\gamma+\delta \rho \\
& \lambda_{n}^{Y}=\gamma \rho+\delta .
\end{aligned}
$$

Se $\alpha$ é não-nula, então $1-\rho^{2} \neq 0$. Assim, segue de (3.27) que

$$
\left\{\begin{array}{l}
\gamma=\frac{\lambda_{n}^{X}-\rho \lambda_{n}^{Y}}{1-\rho^{2}} \\
\delta=\frac{\lambda_{n}^{Y}-\rho \lambda_{n}^{X}}{1-\rho^{2}} .
\end{array}\right.
$$

Portanto, segue das equações (3.24), (3.25), (3.26) e (3.27) a seguinte proposição.

Proposição 3.5. Suponha que $\alpha$ seja a curva de interseção não-nula das duas superfícies tipo tempo X e Y. Então, sua torção $\tau$ é dada por

1. Se a é tipo tempo:

$$
\tau=\frac{\left(\lambda_{n}^{X}-\rho \lambda_{n}^{Y}\right)\left\langle N^{X}, b\right\rangle_{1}+\left(\lambda_{n}^{Y}-\rho \lambda_{n}^{X}\right)\left\langle N^{Y}, b\right\rangle_{1}}{\kappa\left(1-\rho^{2}\right)} .
$$

2. Se $\alpha$ e $\alpha^{\prime \prime}$ são tipo espaço:

$$
\tau=-\frac{\left(\lambda_{n}^{X}-\rho \lambda_{n}^{Y}\right)\left\langle N^{X}, b\right\rangle_{1}+\left(\lambda_{n}^{Y}-\rho \lambda_{n}^{X}\right)\left\langle N^{Y}, b\right\rangle_{1}}{\kappa\left(1-\rho^{2}\right)} .
$$

3. Se a é tipo espaço e $\alpha^{\prime \prime}$ é tipo tempo:

$$
\tau=\frac{\left(\lambda_{n}^{X}-\rho \lambda_{n}^{Y}\right)\left\langle N^{X}, b\right\rangle_{1}+\left(\lambda_{n}^{Y}-\rho \lambda_{n}^{X}\right)\left\langle N^{Y}, b\right\rangle_{1}}{\kappa\left(1-\rho^{2}\right)} .
$$

4. Se $\alpha$ é tipo espaço e $\alpha^{\prime \prime}$ é tipo luz:

$$
\tau=\frac{\left(\lambda_{n}^{X}-\rho \lambda_{n}^{Y}\right)\left\langle N^{X}, b\right\rangle_{1}+\left(\lambda_{n}^{Y}-\rho \lambda_{n}^{X}\right)\left\langle N^{Y}, b\right\rangle_{1}}{1-\rho^{2}} .
$$

Em particular, veremos o que acontece com a curvatura $\kappa$ e a torção $\tau$ quando $\alpha$ é tipo tempo. 
Sejam $X$ e $Y$ duas superfícies paramétricas tipo tempo em $\mathbb{L}^{3}$ com vetores normais $N^{X}$ e $N^{Y}$ e $\alpha$ a curva de interseção transversal entre elas, a qual supomos ser tipo tempo. Logo, $t$ é tipo tempo e, como $n, b \in \operatorname{Span}\{t\}^{\perp}$, então $n$ e $b$ são tipo espaço. Assim, sendo $n \perp b$, tem-se que o plano normal Span $\{n, b\}$ é tipo espaço. Por outro lado, como $\left\langle N^{X}, N^{Y}\right\rangle_{1}=\rho \in(-1,1)$ (ver Proposição 3.3), existe um $\theta \in(0, \pi)$ tal que

$$
\left\langle N^{X}, N^{Y}\right\rangle_{1}=\cos \theta
$$

Portanto, da equação (3.21) e das equações (3.28) para $\alpha$ tipo tempo, segue:

Corolário 3.1. Sejam X e Y superfícies paramétricas tipo tempo em $\mathbb{L}^{3}$ e $\alpha$ a curva de interseção transversal tipo tempo.

1. A curvatura e a torção de $\alpha$ são dadas por

$$
\begin{gathered}
\kappa^{2}=\frac{\left|\left(\kappa_{n}^{X}\right)^{2}+\left(\kappa_{n}^{Y}\right)^{2}-2 \cos \theta \kappa_{n}^{X} \kappa_{n}^{Y}\right|}{\sin ^{2} \theta} \\
\tau=\frac{\left(\lambda_{n}^{X}-\cos \theta \lambda_{n}^{Y}\right)\left\langle N^{X}, b\right\rangle_{1}+\left(\lambda_{n}^{Y}-\cos \theta \lambda_{n}^{X}\right)\left\langle N^{Y}, b\right\rangle_{1}}{\kappa \sin ^{2} \theta} .
\end{gathered}
$$

2. $\operatorname{Se} \theta=\frac{\pi}{2}$, então

$$
\begin{gathered}
\kappa^{2}=\left(\kappa_{n}^{X}\right)^{2}+\left(\kappa_{n}^{Y}\right)^{2} \\
\tau=\frac{\lambda_{n}^{X}\left\langle N^{X}, b\right\rangle_{1}+\lambda_{n}^{Y}\left\langle N^{Y}, b\right\rangle_{1}}{\kappa} .
\end{gathered}
$$

Com isto, finalizamos o estudo das curvas de interseção transversal entre superfícies paramétricas no espaço de Lorentz-Minkowski. 


\section{Capítulo 4}

\section{Exemplos}

Para ilustrar a teoria e os métodos desenvolvidos para o cálculo das propriedades geométricas da curva de interseção, apresentaremos a seguir alguns exemplos no espaço Euclidiano e no espaço de Lorentz-Minkowski, considerando cada caso tratado nos capítulos anteriores.

\subsection{Exemplos no Espaço Euclidiano}

Exemplo 4.1 (Interseção entre duas Superfícies Paramétricas). Considere os hiperboloides de uma folha

$$
\begin{aligned}
& X(u, v)=(\cos u-v \sin u, \sin u+v \cos u, v) \\
& Y(u, v)=(-v, \sin u+v \cos u, \cos u-v \sin u) .
\end{aligned}
$$

Vamos analisar a interseção tangencial entre estas superfícies. Note que

$$
\begin{array}{ll}
X_{u}=(-\sin u-v \cos u, \cos u-v \sin u, 0) & X_{u u}=(-\cos u+v \sin u,-\sin u-v \cos u, 0) \\
X_{v}=(-\sin u, \cos u, 1) & X_{u v}=(-\cos u,-\sin u, 0) \\
X_{u} \wedge X_{v}=(\cos u-v \sin u, \sin u+v \cos u,-v) & X_{v v}=0
\end{array}
$$

$$
\begin{array}{ll}
Y_{u}=(0, \cos u-v \sin u,-\sin u-v \cos u) & Y_{u u}=(0,-\sin u-v \cos u,-\cos u+v \sin u) \\
Y_{v}=(-1, \cos u,-\sin u) & Y_{u v}=(0,-\sin u,-\cos u) \\
Y_{u} \wedge Y_{v}=(v, \sin u+v \cos u, \cos u-v \sin u) & Y_{v v}=0
\end{array}
$$




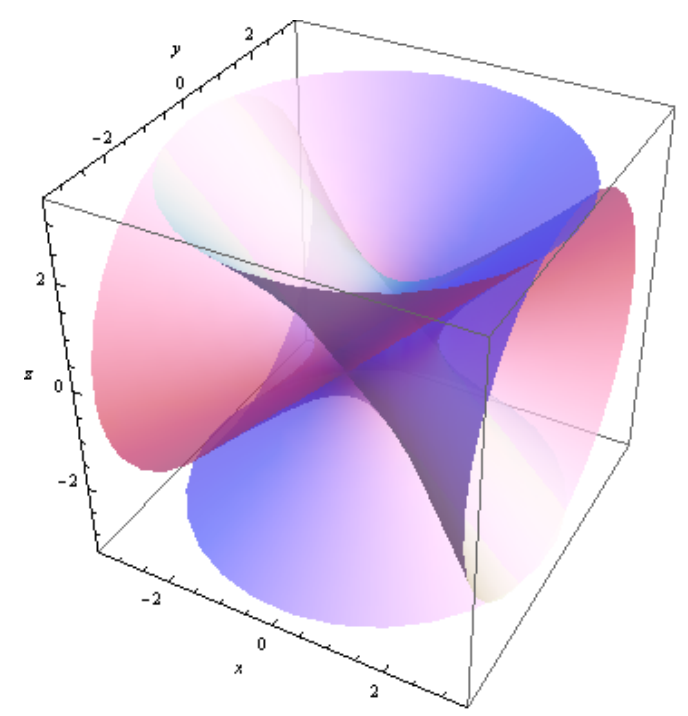

Figura 4.1: Interseção das superfícies paramétricas $X(u, v)$ e $Y(u, v)$

$$
\begin{aligned}
& N^{X}=\frac{(\cos u-v \sin u, \sin u+v \cos u,-v)}{\sqrt{1+2 v^{2}}} \\
& N^{Y}=\frac{(v, \sin u+v \cos u, \cos u-v \sin u)}{\sqrt{1+2 v^{2}}} .
\end{aligned}
$$

Portanto, $N^{X}=N^{Y}$ se, e somente se, $(u, v)=\left( \pm \frac{\pi}{2}, 0\right)$, o que mostra que os pontos de interseção tangencial são $P=(0,1,0)$ e $P^{\prime}=(0,-1,0)$. Faremos uma análise para o ponto $P=(0,1,0)$ e todas as funções a seguir são avaliadas neste ponto. Pelas equações (4.1) e (4.2) (aplicadas a $P$ ) concluímos que

$$
\begin{array}{lll}
X_{u}=(-1,0,0) & X_{u u}=X_{u v}=(0,-1,0) & X_{u u u}=X_{u u v}=(1,0,0) \\
X_{v}=(-1,0,1) & X_{v v}=(0,0,0) & X_{u v v}=X_{v v v}=(0,0,0) \\
X_{u u u u}=X_{u u u v}=(0,1,0) & X_{u u v v}=X_{u v v v}=X_{v v v v}=(0,0,0) & N^{X}=(0,1,0), \\
& Y_{u u}=Y_{u v}=(0,-1,0) & Y_{u u u}=Y_{u u v}=(0,0,1) \\
Y_{u}=(0,0,-1) & Y_{v v}=(0,0,0) & Y_{u v v}=Y_{v v v}=(0,0,0) \\
Y_{v}=(-1,0,-1) & Y_{u u v v}=Y_{u v v v}=Y_{v v v v}=(0,0,0) & N^{Y}=(0,1,0) .
\end{array}
$$

Logo,

$$
\begin{aligned}
& E^{X}=E^{Y}=1, F^{X}=F^{Y}=1, G^{X}=G^{Y}=2 \\
& e^{X}=e^{Y}=-1, f^{X}=f^{Y}=-1, g^{X}=g^{Y}=0 .
\end{aligned}
$$


Assim, pelas equações (2.51) e (2.53), tem-se

$$
\begin{aligned}
& a_{11}=-1, a_{12}=-2, a_{21}=1, a_{22}=1 \Rightarrow \\
& b_{11}=2, b_{12}=2, b_{22}=0 \Rightarrow \\
& \Delta=4\left(b_{12}^{2}-b_{11} b_{22}\right)>0,
\end{aligned}
$$

ou seja, $P$ é ponto de ramificação e a equação (2.54) tem duas soluções, que serão denotadas por $\omega_{1}$ e $\omega_{2}$ :

$$
\omega_{1}=\frac{-b_{12}-\sqrt{b_{12}^{2}-b_{11} b_{22}}}{b_{11}}=-2, \quad \omega_{2}=\frac{-b_{12}+\sqrt{b_{12}^{2}-b_{11} b_{22}}}{b_{11}}=0 .
$$

Portanto, pelo Teorema 2.8, os vetores tangentes em $P$ relativos às soluções $\omega_{1}$ e $\omega_{2}$ são dados por

$$
\begin{aligned}
& t_{1}=\frac{\omega_{1} X_{u}+X_{v}}{\left\|\omega_{1} X_{u}+X_{v}\right\|}=\frac{-2 X_{u}+X_{v}}{\left\|-2 X_{u}+X_{v}\right\|}=\frac{(1,0,1)}{\|(1,0,1)\|}=\left(\frac{\sqrt{2}}{2}, 0, \frac{\sqrt{2}}{2}\right) \\
& t_{2}=\frac{\omega_{2} X_{u}+X_{v}}{\left\|\omega_{2} X_{u}+X_{v}\right\|}=\frac{X_{v}}{\left\|X_{v}\right\|}=\frac{(-1,0,1)}{\|(-1,0,1)\|}=\left(-\frac{\sqrt{2}}{2}, 0, \frac{\sqrt{2}}{2}\right) .
\end{aligned}
$$

Até aqui, mostramos que $P=(0,1,0)$ é um ponto de ramificação com os vetores tangentes $t_{1}$ e $t_{2}$ definidos neste ponto, isto é, existem dois ramos de interseção, digamos, $\alpha_{1}$ e $\alpha_{2}$, tais que, em $P, \alpha_{1}^{\prime}=t_{1}$ e $\alpha_{2}^{\prime}=t_{2}$. Precisamos encontrar as curvaturas de $\alpha_{1}$ e $\alpha_{2}$ em $P$. Vamos fazer as contas para $\alpha_{1}$ usando o método descrito na Subseção 2.2.1. Alguns coeficientes ainda precisam ser calculados. Denotando $t_{1}=u_{1}^{\prime} X_{u}+v_{1}^{\prime} X_{v}=u_{2}^{\prime} Y_{u}+v_{2}^{\prime} Y_{v}$, então sendo $t_{1}=-\sqrt{2} X_{u}+\frac{\sqrt{2}}{2} X_{v}$, temos que

$$
u_{1}^{\prime}=-\sqrt{2}, \quad v_{1}^{\prime}=\frac{\sqrt{2}}{2}
$$

e, pela equação (2.50), tem-se

$$
u_{2}^{\prime}=0, \quad v_{2}^{\prime}=-\frac{\sqrt{2}}{2} .
$$

Portanto, por (2.58), (2.59) e (2.60),

$$
\Lambda=0, \quad a_{13}=a_{23}=0, \quad u_{2}^{\prime \prime}=-u_{1}^{\prime \prime}-2 v_{1}^{\prime \prime}, \quad v_{2}^{\prime \prime}=u_{1}^{\prime \prime}+v_{1}^{\prime \prime} .
$$

Além disso, pelas equações (2.61) e (2.62), $u_{1}^{\prime \prime}=v_{1}^{\prime \prime}=0$. Substituindo estes valores na primeira igualdade das equações (2.56), concluímos que

$$
\vec{K}=(0,0,0), \quad \kappa=\sqrt{\langle\vec{K}, \vec{K}\rangle}=0
$$


Neste caso, sendo $\kappa=0$, não podemos calcular a torção com o método descrito na Subseção 2.2.1. No entanto, vamos calcular o vetor $\alpha^{\prime \prime \prime}$ para exemplificar o método. Para isto, resta-nos conhecer $u_{1}^{\prime \prime \prime}$ e $v_{1}^{\prime \prime \prime}$. Das equações (1.4), tem-se

$$
\begin{aligned}
\alpha^{\prime \prime \prime}= & X_{u_{1} u_{1} u_{1}}\left(u_{1}^{\prime}\right)^{3}+3 X_{u_{1} u_{1} v_{1}}\left(u_{1}^{\prime}\right)^{2} v_{1}^{\prime}+3 X_{u_{1} v_{1} v_{1}} u_{1}^{\prime}\left(v_{1}^{\prime}\right)^{2}+X_{v_{1} v_{1} v_{1}}\left(v_{1}^{\prime}\right)^{3}+ \\
& 3\left[X_{u_{1} u_{1}} u_{1}^{\prime} u_{1}^{\prime \prime}+X_{u_{1} v_{1}}\left(u_{1}^{\prime \prime} v_{1}^{\prime}+u_{1}^{\prime} v_{1}^{\prime \prime}\right)+X_{v_{1} v_{1}} v_{1}^{\prime} v_{1}^{\prime \prime}\right]+X_{u_{1}} u_{1}^{\prime \prime \prime}+X_{v_{1}} v_{1}^{\prime \prime \prime} \\
= & Y_{u_{2} u_{2} u_{2}}\left(u_{2}^{\prime}\right)^{3}+3 Y_{u_{2} u_{2} v_{2}}\left(u_{2}^{\prime}\right)^{2} v_{2}^{\prime}+3 Y_{u_{2} v_{2} v_{2}} u_{2}^{\prime}\left(v_{2}^{\prime}\right)^{2}+Y_{v_{2} v_{2} v_{2}}\left(v_{2}^{\prime}\right)^{3}+ \\
& 3\left[Y_{u_{2} u_{2}} u_{2}^{\prime} u_{2}^{\prime \prime}+Y_{u_{2} v_{2}}\left(u_{2}^{\prime \prime} v_{2}^{\prime}+u_{2}^{\prime} v_{2}^{\prime \prime}\right)+Y_{v_{2} v_{2}} v_{2}^{\prime} v_{2}^{\prime \prime}\right]+Y_{u_{2}} u_{2}^{\prime \prime \prime}+Y_{v_{2}} v_{2}^{\prime \prime \prime} .
\end{aligned}
$$

Logo,

$$
X_{u_{1}} u_{1}^{\prime \prime \prime}+X_{v_{1}} v_{1}^{\prime \prime \prime}=Y_{u_{2}} u_{2}^{\prime \prime \prime}+Y_{v_{2}} v_{2}^{\prime \prime \prime}+\Gamma
$$

onde

$$
\begin{aligned}
\Gamma & =Y_{u_{2} u_{2} u_{2}}\left(u_{2}^{\prime}\right)^{3}+3 Y_{u_{2} u_{2} v_{2}}\left(u_{2}^{\prime}\right)^{2} v_{2}^{\prime}+3 Y_{u_{2} v_{2} v_{2}} u_{2}^{\prime}\left(v_{2}^{\prime}\right)^{2}+Y_{v_{2} v_{2} v_{2}}\left(v_{2}^{\prime}\right)^{3} \\
& +3\left[Y_{u_{2} u_{2}} u_{2}^{\prime} u_{2}^{\prime \prime}+Y_{u_{2} v_{2}}\left(u_{2}^{\prime \prime} v_{2}^{\prime}+u_{2}^{\prime} v_{2}^{\prime \prime}\right)+Y_{v_{2} v_{2}} v_{2}^{\prime} v_{2}^{\prime \prime}\right] \\
& -\left[X_{u_{1} u_{1} u_{1}}\left(u_{1}^{\prime}\right)^{3}+3 X_{u_{1} u_{1} v_{1}}\left(u_{1}^{\prime}\right)^{2} v_{1}^{\prime}+3 X_{u_{1} v_{1} v_{1}} u_{1}^{\prime}\left(v_{1}^{\prime}\right)^{2}+X_{v_{1} v_{1} v_{1}}\left(v_{1}^{\prime}\right)^{3}\right. \\
& \left.+3\left[X_{u_{1} u_{1}} u_{1}^{\prime} u_{1}^{\prime \prime}+X_{u_{1} v_{1}}\left(u_{1}^{\prime \prime} v_{1}^{\prime}+u_{1}^{\prime} v_{1}^{\prime \prime}\right)+X_{v_{1} v_{1}} v_{1}^{\prime} v_{1}^{\prime \prime}\right]\right] .
\end{aligned}
$$

Tomemos o produto vetorial de (4.4) por $Y_{u_{2}}$ e $Y_{v_{2}}$ e o produto interno da equação resultante por $N$, obtendo:

$$
\begin{aligned}
& u_{2}^{\prime \prime \prime}=a_{11} u_{1}^{\prime \prime \prime}+a_{12} v_{1}^{\prime \prime \prime}+c_{1} \\
& v_{2}^{\prime \prime \prime}=a_{21} u_{1}^{\prime \prime \prime}+a_{22} v_{1}^{\prime \prime \prime}+c_{2}
\end{aligned}
$$

onde os coeficientes $a_{i j}(i, j=1,2)$ são dados por (2.51) e

$$
c_{1}=-\frac{\operatorname{det}\left(\Gamma, Y_{v_{2}}, N\right)}{\sqrt{E^{Y} G^{Y}-\left(F^{Y}\right)^{2}}}, \quad c_{2}=-\frac{\operatorname{det}\left(Y_{u_{2}}, \Gamma, N\right)}{\sqrt{E^{Y} G^{Y}-\left(F^{Y}\right)^{2}}} .
$$

Note que, no ponto $P, \Gamma=(-\sqrt{2}, 0,0)$. Logo, $c_{1}=\sqrt{2}$ e $c_{2}=-\sqrt{2}$. Portanto,

$$
\begin{aligned}
& u_{2}^{\prime \prime \prime}=-u_{1}^{\prime \prime \prime}-2 v_{1}^{\prime \prime \prime}+\sqrt{2} \\
& v_{2}^{\prime \prime \prime}=u_{1}^{\prime \prime \prime}+v_{1}^{\prime \prime \prime}-\sqrt{2}
\end{aligned}
$$

Derivando (4.3), fazendo o produto interno por $N$ e aplicando o resultado no ponto $P$, obtemos o escalar $\lambda_{n}=\left\langle\alpha^{(4)}, N\right\rangle$. Fazendo-se $\lambda_{n}^{X}=\lambda_{n}^{Y}$, segue que

$$
\sqrt{2} u_{1}^{\prime \prime \prime}+2 \sqrt{2} v_{1}^{\prime \prime \prime}-2=0 .
$$


Uma outra equação envolvendo $u_{1}^{\prime \prime \prime}$ e $v_{1}^{\prime \prime \prime}$ pode ser obtida escrevendo-se $\alpha^{\prime \prime \prime}$ na base $\{t, n, b\}$ : derivando $\alpha^{\prime \prime}=\kappa n$ (ver equações (1.1)), obtemos $\alpha^{\prime \prime \prime}=-\kappa^{2} t+\kappa^{\prime} n+\kappa \tau b$. Logo, $\left\langle\alpha^{\prime \prime \prime}, t\right\rangle=-\kappa^{2}$. Vimos que $\kappa=0$ em $P$. Assim, $\left\langle\alpha^{\prime \prime \prime}, t\right\rangle=0$. Por outro lado, da primeira igualdade em (4.3), temos que no ponto $P$ vale $\left\langle\alpha^{\prime \prime \prime}, t\right\rangle=-\frac{\sqrt{2}}{2} u_{1}^{\prime \prime \prime}+1$. Logo, $-\frac{\sqrt{2}}{2} u_{1}^{\prime \prime \prime}+1=0$, o que implica $u_{1}^{\prime \prime \prime}=\sqrt{2}$. Substituindo este valor na equação (4.5), segue que $v_{1}^{\prime \prime \prime}=0$. Assim, pela primeira igualdade na equação $(4.3), \alpha^{\prime \prime \prime}=(0,0,0)$.

Exemplo 4.2 (Interseção entre Superfícies Paramétricas e Implícitas). Vamos considerar a interseção entre um cilindro e uma esfera, ambos de raio três, dados respectivamente por

$$
\begin{aligned}
X(u, v) & =(u, 3 \sin v, 3 \cos v), 0<v<2 \pi \\
f(x, y, z) & =x^{2}+y^{2}+z^{2}-9=0 .
\end{aligned}
$$

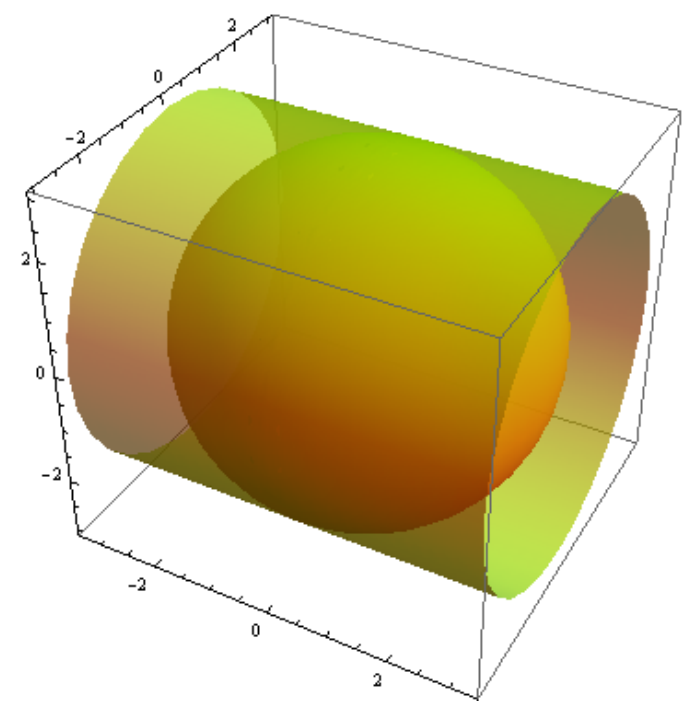

Figura 4.2: Interseção entre as superfícies paramétrica e implícita $X(u, v)$ e $f(x, y, z)$

Temos que

$$
\begin{array}{cll}
X_{u}=(1,0,0) & X_{u u}=X_{u v}=0 & X_{u u u}=X_{u u v}=X_{u v v}=0 \\
X_{v}=(0,3 \cos v,-3 \sin v) & X_{v v}=(0,-3 \sin v,-3 \cos v) & X_{v v v}=(0,-3 \cos v, 3 \sin v), \\
f_{x}=2 x & f_{y}=2 y \quad f_{z}=2 z & f_{x x}=f_{y y}=f_{z z}=2
\end{array}
$$

e as derivadas de segunda e terceira ordem são todas nulas.

$$
\begin{array}{ll}
\nabla f=2(x, y, z) & X_{u} \wedge X_{v}=(0,3 \sin v, 3 \cos v) \\
\|\nabla f\|=6 & \left\|X_{u} \wedge X_{v}\right\|=3 \\
N^{f}=\frac{1}{3}(x, y, z) & N^{X}=(0, \sin v, \cos v) .
\end{array}
$$


Portanto, $N^{f}=N^{X}$ se, e somente se,

$$
\left\{\begin{array}{l}
x=0 \\
y=3 \sin v \\
z=3 \cos v
\end{array}\right.
$$

ou seja, a interseção tangencial é dada ao longo de um círculo de raio 3 no plano yOz. Poderíamos ter verificado que a interseção tangencial se dá ao longo de uma curva observando que, pelas equações (2.64), $b_{11} \equiv 2, b_{12}=b_{22} \equiv 0$ e, portanto, $\Delta=0$. Agora, como

$$
\omega=\frac{-b_{12} \pm \sqrt{b_{12}^{2}-b_{11} b_{22}}}{b_{11}}=0,
$$

então

$$
t=\frac{\omega X_{u}+X_{v}}{\left\|\omega X_{u}+X_{v}\right\|}=\frac{X_{v}}{\left\|X_{v}\right\|}=(0, \cos v,-\sin v)
$$

Note que, por (2.65),

$$
\begin{aligned}
& v^{\prime}=-\left(E \omega^{2}+2 F \omega+G\right)^{-\frac{1}{2}}=-\frac{1}{3} \\
& u^{\prime}=\omega v^{\prime}=0 .
\end{aligned}
$$

Para calcular o vetor curvatura e a curvatura, não poderemos usar o método apresentado na Subseção 2.2.2, visto que $b_{11} \omega+b_{12}=0$. Mas, observando que as contas feitas são para pontos sobre uma curva de interseção e não para apenas um ponto, podemos derivar $u^{\prime}$ e $v^{\prime}$ para obter $u^{\prime \prime}$ e $v^{\prime \prime}$. Assim, por (4.7), $u^{\prime \prime}=v^{\prime \prime} \equiv 0$ e, pelas equações (1.4) e (4.6), o vetor curvatura é dado por $\vec{K}=-\frac{1}{3}(0, \sin v, \cos v)$ e a curvatura é

$$
\kappa=\sqrt{\langle\vec{K}, \vec{K}\rangle}=\frac{1}{3}
$$

Por fim, derivando $u^{\prime \prime}$ e $v^{\prime \prime}$, tem-se $u^{\prime \prime \prime}=v^{\prime \prime \prime} \equiv 0$. Assim, das equações (1.4) e (4.6), segue que $\alpha^{\prime \prime \prime}=\frac{1}{9}(0,-\cos v, \sin v)$. Além disso,

$$
b=t \wedge n=t \wedge \frac{\vec{K}}{\kappa}=(-1,0,0) .
$$

Portanto, a torção é

$$
\tau=\frac{\left\langle b, \alpha^{\prime \prime \prime}\right\rangle}{\kappa}=0
$$

Podemos observar que não há interseção transversal, pois os pontos de interseção de $f$ e $X$ são aqueles que satisfazem $f(X(u, v))=0$, isto é, os pontos $X(0, v)=(0,3 \sin v, 3 \cos v)$, que são todos tangenciais, como visto anteriormente. 
Exemplo 4.3 (Interseção entre duas Superfícies Implícitas). Considere as superfícies implícitas (esfera e plano)

$$
\begin{aligned}
& f(x, y, z)=x^{2}+y^{2}+z^{2}-1=0 \\
& h(x, y, z)=y-1=0 .
\end{aligned}
$$

Note que

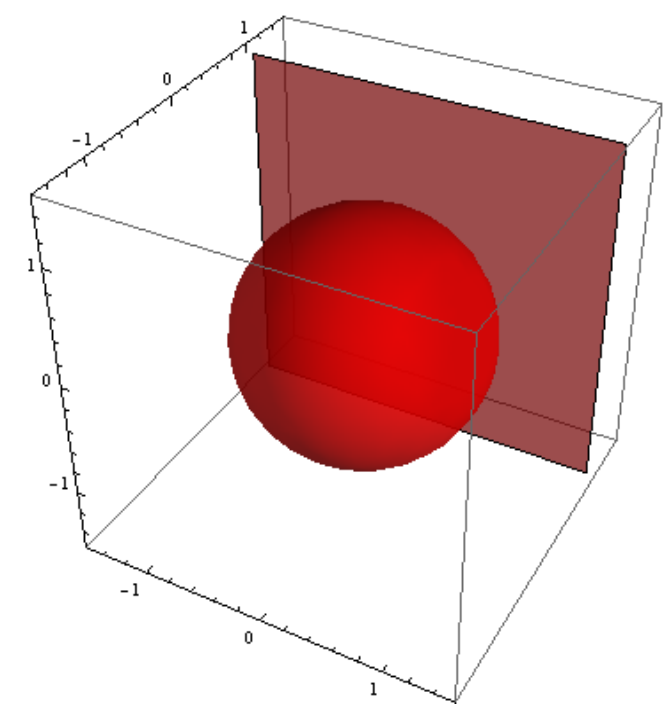

Figura 4.3: Interseção entre as superfícies implícitas $f(x, y, z)$ e $h(x, y, z)$

$$
\begin{array}{ll}
f_{x}=2 x \quad f_{x x}=f_{y y}=f_{z z}=2 \\
f_{y}=2 y \quad f_{x y}=f_{x z}=f_{y z}=0 \\
f_{z}=2 z \quad N^{f}=(x, y, z), \\
h_{x}=h_{z}=0 & h_{x x}=h_{y y}=h_{z z}=h_{x y}=h_{x z}=h_{y z}=0 \\
h_{y}=1 & N^{h}=(0,1,0) .
\end{array}
$$

Logo, $N^{f}=N^{h}$ se, e somente se, $(x, y, z)=(0,1,0)$. Em $P=(0,1,0)$, temos que $A=\frac{\|\nabla f\|}{\|\nabla h\|}=2$ e, pela equação (2.72),

$$
b_{11}=8, \quad b_{12}=0, \quad b_{22}=8,
$$

o que implica $\Delta=4\left(b_{12}^{2}-b_{11} b_{22}\right)<0$. Portanto, $P$ é ponto de contato isolado.

Observe que o ponto $P=(0,1,0)$, que é de interseção tangencial, é o único ponto na interseção de $f$ e $h$. Logo, não há pontos de interseção transversal. 


\subsection{Exemplos no Espaço de Lorentz-Minkowski}

Exemplo 4.4 (Interseção Transversal entre Superfícies Tipo Espaço). Seja

$$
X(u, v)=(\sinh u, v, \cosh u)
$$

uma superfície paramétrica no espaço de Lorentz-Minkowski (cilindro hiperbólico). O vetor normal de $X$ é, então, dado por $N^{X}=(-\sinh u, 0,-\cosh u)$. Considerando a curva $\alpha(s)=$ $(\sinh s, 0, \cosh s)$ sobre $X$, temos que $t(s)=\alpha^{\prime}(s)=(\cosh s, 0, \sinh s)$ e $V^{X}(s)=N^{X}(s) \wedge_{1}$ $t(s)=(0,-1,0)$. Assim, defina a superfície regrada

$$
Y(s, w)=\alpha(s)+w \beta(s), \quad w \in(-2,2)
$$

onde $\beta(s)=\frac{N^{X}}{2}+V^{X}$. Escrevendo de outra forma, temos que

$$
Y(s, w)=\left(\left(1-\frac{w}{2}\right) \sinh s,-w,\left(1-\frac{w}{2}\right) \cosh s\right) .
$$

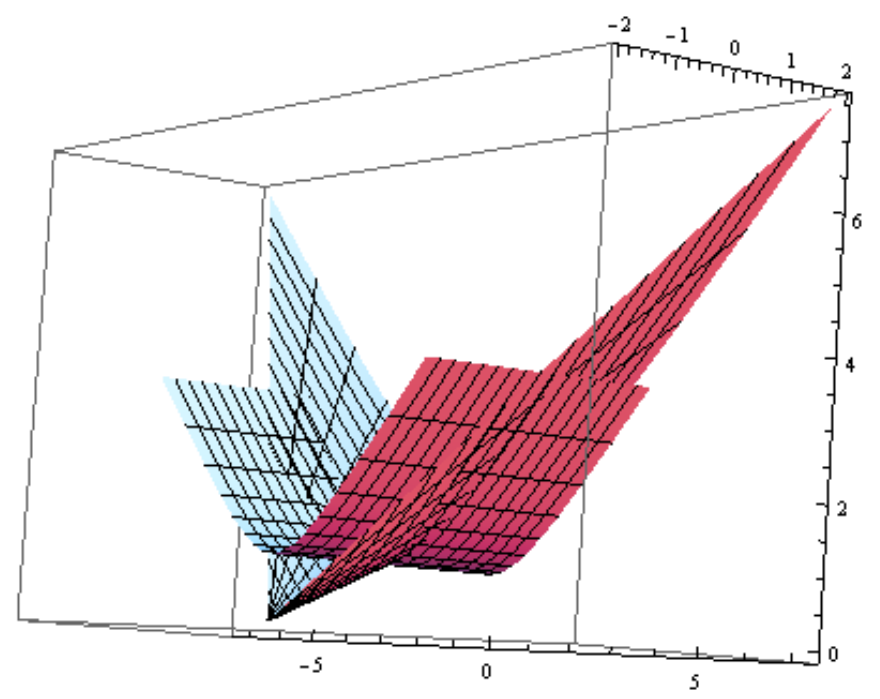

Figura 4.4: Interseção entre as superfícies paramétricas tipo espaço $X(u, v)$ e $Y(s, w)$

Note que $\alpha$ é a curva de interseção entre $X$ e $Y$. Temos ainda que

$$
Y_{s}=\left(\left(1-\frac{w}{2}\right) \cosh s, 0,\left(1-\frac{w}{2}\right) \sinh s\right), \quad Y_{w}=\left(-\frac{\sinh s}{2},-1,-\frac{\cosh s}{2}\right)
$$

e, portanto,

$$
N^{Y}=\frac{2}{\sqrt{3}}\left(\sinh s, \frac{1}{2}, \cosh s\right)
$$


Como $\left\langle N^{X}, N^{X}\right\rangle_{1}=\left\langle N^{Y}, N^{Y}\right\rangle_{1}=-1<0$, então $N^{X}$ e $N^{Y}$ são vetores tipo tempo, o que implica que $X$ e $Y$ são superfícies tipo espaço. Além disso, a torção geodésica de $X$ é

$$
\tau_{g}^{X}=\left\langle\left(N^{X}\right)^{\prime}(s), V^{X}(s)\right\rangle_{1}=0
$$

e, portanto, $\alpha$ é uma linha de curvatura de $X$. Os normais $N^{X}$ e $N^{Y}$ não estão no mesmo cone tipo tempo, visto que $\left\langle N^{X}, N^{Y}\right\rangle_{1}=\frac{2}{\sqrt{3}}>0$ e, assim, o ângulo hiperbólico entre $N^{X}$ e $N^{Y}$ é o número real $\varphi$ que satisfaz

$$
\cosh \varphi=\frac{\left\langle N^{X}, N^{Y}\right\rangle_{1}}{\left\|N^{X}\right\|_{1}\left\|N^{Y}\right\|_{1}}=\frac{2}{\sqrt{3}}
$$

ou seja, $\varphi$ é constante. Por fim, sendo

$$
\left(N^{Y}\right)^{\prime}(s)=\frac{2}{\sqrt{3}}(\cosh s, 0, \sinh s), \quad V^{Y}(s)=\frac{1}{\sqrt{3}}(\sinh s, 2, \cosh s),
$$

então

$$
\tau_{g}^{Y}=\left\langle\left(N^{Y}\right)^{\prime}, V^{Y}\right\rangle_{1}=0
$$

isto é, $\alpha$ é linha de curvatura de $Y$. Este exemplo ilustra o Teorema 3.2.

Exemplo 4.5 (Interseção Transversal entre Superfícies Tipo Tempo). Considere as superfícies paramétricas (cilindro hiperbólico e plano)

$$
\begin{aligned}
& X\left(u_{1}, v_{1}\right)=\left(v_{1}, \cosh u_{1}, \sinh u_{1}\right) \\
& Y\left(u_{2}, v_{2}\right)=\left(0, u_{2}, v_{2}\right) .
\end{aligned}
$$

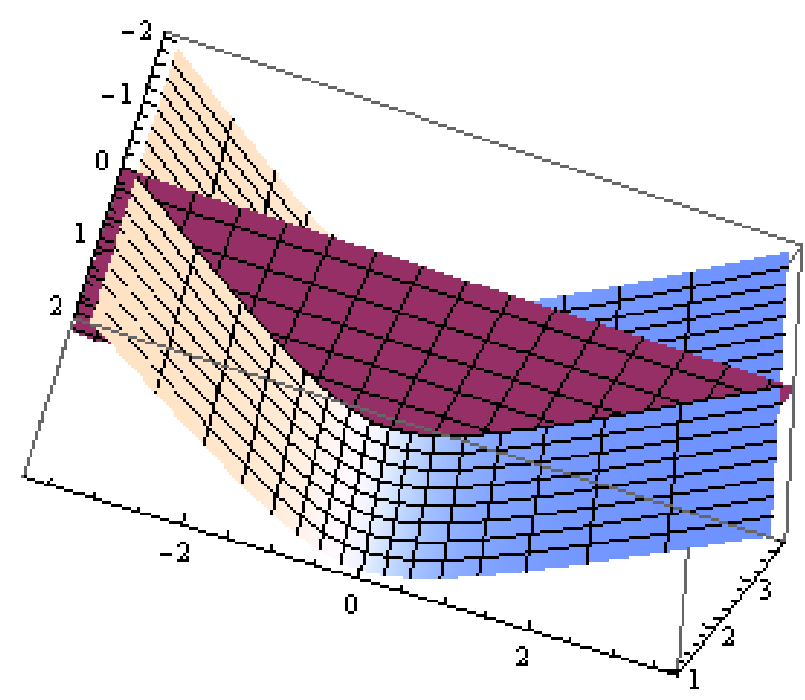

Figura 4.5: Interseção entre as superfícies paramétricas tipo tempo $X\left(u_{1}, v_{1}\right)$ e $Y\left(u_{2}, v_{2}\right)$ 
Note que

$$
\begin{array}{ll}
X_{u_{1}}=\left(0, \sinh u_{1}, \cosh u_{1}\right) & Y_{u_{2}}=(0,1,0) \\
X_{v_{1}}=(1,0,0) & Y_{v_{2}}=(0,0,1) \\
X_{u_{1}} \wedge_{1} X_{v_{1}}=\left(0, \cosh u_{1}, \sinh u_{1}\right) & Y_{u_{2}} \wedge_{1} Y_{v_{2}}=(1,0,0) .
\end{array}
$$

Portanto,

$$
N^{X}=\left(0, \cosh u_{1}, \sinh u_{1}\right), \quad N^{Y}=(1,0,0) .
$$

Como $\left\langle N^{X}, N^{X}\right\rangle_{1}=\left\langle N^{Y}, N^{Y}\right\rangle_{1}=1>0$, segue que os normais $N^{X}$ e $N^{Y}$ são tipo espaço e, portanto, $X$ e $Y$ são superfícies tipo tempo. Além disso,

$$
\rho=\left\langle N^{X}, N^{Y}\right\rangle_{1}=0 \in(-1,1) .
$$

Logo, pela Proposição 3.3, a curva de interseção é tipo tempo. Denote por $\alpha(s)$ tal curva. Pela equação (3.16), segue que

$$
t(s)=\frac{N^{X} \wedge_{1} N^{Y}}{\left\|N^{X} \wedge_{1} N^{Y}\right\|_{1}}(s)=(0, \sinh s, \cosh s) .
$$

Vamos calcular a curvatura e a torção de $\alpha$, as quais são dadas em termos das curvaturas normais $\kappa_{n}^{X}$ e $\kappa_{n}^{Y}$. Para calcular $\kappa_{n}^{X}$ e $\kappa_{n}^{Y}$, utilizamos o método descrito na Seção 2.1, isto é,

$$
\kappa_{n}^{X}=e_{1}^{X}\left(u_{1}^{\prime}\right)^{2}+2 f_{1}^{X} u_{1}^{\prime} v_{1}^{\prime}+g_{1}^{X}\left(v_{1}^{\prime}\right)^{2},
$$

onde

$$
u_{1}^{\prime}=\frac{\left\langle t, X_{u_{1}}\right\rangle_{1} G_{1}^{X}-\left\langle t, X_{v_{1}}\right\rangle_{1} F_{1}^{X}}{E_{1}^{X} G_{1}^{X}-\left(F_{1}^{X}\right)^{2}}, \quad v_{1}^{\prime}=\frac{\left\langle t, X_{v_{1}}\right\rangle_{1} E_{1}^{X}-\left\langle t, X_{u_{1}}\right\rangle_{1} F_{1}^{X}}{E_{1}^{X} G_{1}^{X}-\left(F_{1}^{X}\right)^{2}},
$$

$e_{1}^{X}=\left\langle X_{u_{1} u_{1}}, N^{X}\right\rangle_{1}, f_{1}^{X}=\left\langle X_{u_{1} v_{1}}, N^{X}\right\rangle_{1}, g_{1}^{X}=\left\langle X_{v_{1} v_{1}}, N^{X}\right\rangle_{1}$,

$E_{1}^{X}=\left\langle X_{u_{1}}, X_{u_{1}}\right\rangle_{1}, F_{1}^{X}=\left\langle X_{u_{1}}, X_{v_{1}}\right\rangle_{1}, G_{1}^{X}=\left\langle X_{v_{1}}, X_{v_{1}}\right\rangle_{1}$.

Assim, temos que $\kappa_{n}^{X}=1 \mathrm{e}$, analogamente, obtemos $\kappa_{n}^{Y}=0$. Temos ainda que, sendo $\theta$ o ângulo entre $N^{X}$ e $N^{Y}$, então $\cos \theta=\rho=0$ e $\sin ^{2} \theta=1$. Logo, pelo Corolário 3.1,

$$
\kappa=1
$$

Agora, vamos calcular a torção. Primeiramente, encontraremos o vetor binormal $b$. Note que

$$
n=\frac{\vec{K}}{\kappa}=(0, \cosh s, \sinh s) \text {. }
$$

O vetor curvatura $\vec{K}$ foi obtido de maneira análoga ao que fizemos no caso Euclidiano, isto é, (ver equação (2.4)).

$$
\vec{K}=\frac{\kappa_{n}^{X}-\kappa_{n}^{Y} \cos \theta}{\sin ^{2} \theta} N^{X}+\frac{\kappa_{n}^{Y}-\kappa_{n}^{X} \cos \theta}{\sin ^{2} \theta} N^{Y}
$$




\section{Portanto,}

$$
b=t \wedge_{1} n=(-1,0,0) .
$$

Dessa forma, pelo Corolário 3.1, $\tau=-\lambda_{n}^{Y}$. Encontraremos o escalar $\lambda_{n}^{Y}$ de maneira análoga ao que foi feito nas equações (2.10) e (2.11):

$$
\lambda_{n}^{Y}=3\left[e_{1}^{Y} u_{2}^{\prime} u_{2}^{\prime \prime}+f_{1}^{Y}\left(u_{2}^{\prime \prime} v_{2}^{\prime}+u_{2}^{\prime} v_{2}^{\prime \prime}\right)+g_{1}^{Y} v_{2}^{\prime} v_{2}^{\prime \prime}\right]+\tilde{\Lambda}^{Y}=0,
$$

onde

$$
\begin{aligned}
\tilde{\Lambda}^{Y} & =\left\langle Y_{u_{2} u_{2} u_{2}}, N^{Y}\right\rangle\left(u_{2}^{\prime}\right)^{3}+3\left\langle Y_{u_{2} u_{2} v_{2}}, N^{Y}\right\rangle\left(u_{2}^{\prime}\right)^{2} v_{2}^{\prime}+3\left\langle Y_{u_{2} v_{2} v_{2}}, N^{Y}\right\rangle u_{2}^{\prime}\left(v_{2}^{\prime}\right)^{2}+\left\langle Y_{v_{2} v_{2} v_{2}}, N^{Y}\right\rangle\left(v_{2}^{\prime}\right)^{3} \\
& =0 .
\end{aligned}
$$

Portanto,

$$
\tau=0
$$




\section{Referências Bibliográficas}

[1] ABDEL-ALL, N. H., et al. "Intersection curves of two implicit surfaces in $\mathbb{R}^{3}$." Journal of Mathematical and Computational Science, v.2 N.2 (2012): 152-171.

[2] ARAÚJO, P. V. Geometria Diferencial. Coleção Matemática Universitária, SBM, Rio de Janeiro, 2004.

[3] ALÉSSIO, O.; GUADALUPE, I. V. "Determination of a transversal intersection curve of two spacelike surfaces in Lorentz-Minkowski $\mathbb{L}^{3}$." Hadronic Journal, v.30 N.3 (2007): 315342.

[4] ALÉSSIO, O. "Geometria diferencial de curvas de interseção de duas superfícies implícitas." Trends in Applied and Computational Mathematics, v.7 N.2 (2006): 169-178.

[5] DO CARMO, M. P. Geometria Diferencial de Curvas e Superfícies. 3. ed. Projeto Euclides, SBM, Rio de Janeiro, 1988.

[6] JAVALOYES VICTORIA, M. A.; CAJA, M. S. An Introduction to the Lorentzian Geometry and its Applications. RiMa, São Carlos, 2010.

[7] KÜHNEL, W. Differential Geometry: Curves - Surfaces - Manifolds, Student Mathematical Library, vol. 16, 2. ed, American Mathematical Society (2005).

[8] LIMA, E. L. Curso de Análise vol. 2, Projeto Euclides, SBM, Rio de janeiro, 2010.

[9] LÓPEZ, R. “Differential Geometry of Curves and Surfaces in Lorentz-Minkowski Space." International Electronic Journal of Geometry, v.7 N.1 (2014), 44-107.

[10] NABER, G. L. The Geometry of Minkowski Spacetime: An Introduction to the Mathematics of the Special Theory of Relativity. Dover Publications, New York, 2003.

[11] O'NEILL, B. Semi-Riemannian Geometry with Applications to Relativity. Academic press, London, 1983. 
[12] ŞANLI, Z.; YAYLI, Y. “Non-Null Intersection Curves of Timelike Surfaces in LorentzMinkowski 3-Space." International Journal of Engineering and Applied Sciences, v.1 N.3 (2014): 23-26.

[13] SILVA, F. N. Superfícies Regradas Desenvolvíveis Tipo Tempo e Tipo Espaço no Espaço de Minkowski. Dissertação de Mestrado, Universidade de Brasília, Brasil, 2013.

[14] SOLIMAN, M. A. S., et al. "Intersection Curves of Implicit and Parametric Surfaces in $R^{3}$." Applied Mathematics, v.2 N.8 (2011): 1019-1026.

[15] STRUIK, D. J. Lectures on Classical Differential Geometry, 2. ed. Dover Publications, Inc. New York, 1894.

[16] TENENBLAT, K. Introdução à Geometria Diferencial. Ed. UnB, 1988.

[17] ÜNLÜTÜRK, Y.; ÜMIT, Z. S. “On non-unit speed curves in Minkowski 3-space.” Scientia Magna, v.8 N.4 (2012): 66-74.

[18] YE, X.; MAEKAWA T. "Differential geometry of intersection curves of two surfaces." Computer Aided Geometric Design, v.16 N.8 (1999): 767-788. 